Portland State University

PDXScholar

Summer 8-8-2017

\title{
Enhancing Value-Based Healthcare with Reconstructability Analysis: Predicting Risk for Hip and Knee Replacements
}

Cecily Corrine Froemke

Portland State University

Follow this and additional works at: https://pdxscholar.library.pdx.edu/open_access_etds

Part of the Public Health Commons, Rehabilitation and Therapy Commons, and the Surgery Commons Let us know how access to this document benefits you.

Recommended Citation

Froemke, Cecily Corrine, "Enhancing Value-Based Healthcare with Reconstructability Analysis: Predicting Risk for Hip and Knee Replacements" (2017). Dissertations and Theses. Paper 3772.

https://doi.org/10.15760/etd.5656

This Dissertation is brought to you for free and open access. It has been accepted for inclusion in Dissertations and Theses by an authorized administrator of PDXScholar. Please contact us if we can make this document more accessible: pdxscholar@pdx.edu. 
Enhancing Value-Based Healthcare with Reconstructability Analysis:

Predicting Risk for Hip and Knee Replacements

by

Cecily Corrine Froemke

A dissertation submitted in partial fulfillment of the requirements for the degree of

\author{
Doctor of Philosophy \\ in \\ Systems Science
}

\author{
Dissertation Committee: \\ Martin Zwick, Chair \\ Matthew Carlson \\ Neal Wallace \\ Carlos Crespo
}

Portland State University

2017 
(C) 2017 Cecily Corinne Froemke 


\begin{abstract}
Legislative reforms aimed at slowing growth of US healthcare costs are focused on achieving greater value, defined specifically as health outcomes achieved per dollar spent. To increase value while payments are diminishing and tied to individual outcomes, healthcare must improve at predicting risks and outcomes.

One way to improve predictions is through better modeling methods. Current models are predominantly based on logistic regression (LR). This project applied Reconstructability Analysis (RA) to data on hip and knee replacement surgery, and considered whether RA could create useful models of outcomes, and whether these models could produce predictions complimentary to or even stronger than LR models.

RA is a data mining method that searches for relations in data, especially nonlinear and higher ordinality relations, by decomposing the frequency distribution of the data into projections, several of which taken together define a model, which is then assessed for statistical significance. The predictive power of the model is expressed as the percent reduction of uncertainty (Shannon entropy) of the dependent variable (the DV) gained by knowing the values of the predictive independent variables (the IVs).

Results showed that LR and RA gave the same results for equivalent models, and showed that exploratory RA provided better models than LR. Sixteen RA predictive models were then generated across the four DVs: complications, skilled nursing discharge, readmissions, and total cost. While the first three DVs are nominal, RA generated continuous predictions for cost by calculating expected values. Models included novel comorbidity variables and non-hypothesized interaction terms, and often resulted in substantial reductions in uncertainty.


Predictive variables consisted of both delivery system variables and binary patient comorbidity variables. Complications were predicted by the total number of patient comorbidities. Skilled nursing discharges were predicted both by patient-related factors and delivery system variables (location, surgeon volume), suggesting practice patterns influence utilization of skilled nursing facilities. Readmissions were not well predicted, suggesting the data used in this project lacks the right variables or that readmissions are simply unpredictable. Delivery system variables (surgeon, location, and surgeon volume) were found to be the predominant predictors of total cost.

Risk ratios were generated as an additional measure of effect size. These risk ratios were used to classify the IV states of the models as indicating higher or lower risk of adverse outcomes. Some IV states showed nearly $25 \%$ of patients at increased risk, while other IV states showed over $75 \%$ of patients at decreased risk. In real time, such risk predictions could support clinical decision making and custom-tailored utilization of services.

Future research might address the limitations of this project's data and employ additional RA techniques and training-test splits. Implementation of predictive models is also discussed, with considerations for data supply lines, maintenance of models, organizational buy-in, and the acceptance of model output by clinical teams for use in real-time clinical practice.

If outcomes and risk are adequately predicted, areas for potential improvement become clearer, and focused changes can be made to drive improvements in patient care. Better predictions, such as those resulting from the RA methodology, can thus support improvement in value - better outcomes at a lower cost. As reimbursement increasingly 
evolves into value-based programs, understanding the outcomes achieved, and customizing patient care to reduce unnecessary costs while improving outcomes, will be an active area for clinicians, healthcare administrators, researchers, and data scientists for many years to come. 


\section{Dedication}

Dedicated to my mom, Jennifer Jameson, with gratitude.

$$
\text { - iv - }
$$




\section{Acknowledgments}

I would like to thank my teacher and my mentor, Dr. Martin Zwick, for inspiring me to solve difficult problems and never doubting me.

To my friend, Dr. Joe Fusion, for simplifying complexity over many years of inquiry and for his unwavering support and friendship.

To my dad, Brad Froemke, for showing me how to be patient while figuring things out and to find something funny in most everything.

To my awesome committee including Dr. Matt Carlson, Dr. Neal Wallace, and Dr. Carlos Crespo. Their patience, thoughtful feedback, and good humor helped me conclude this long process with positivity and laughter.

To my early academic champions, Dr. Randy Blazak, Dr. Bob Leibman, and Dr. Rick Lockwood. Their recognition of my academic abilities was pivotal in my decision to pursue advanced study.

I would also like to acknowledge Guy Feldman and Lian Wang for statistical consultation and to Dave Donell for his careful review and editing.

To Dr. Phyllis Brown, Douglas Hagen, and Cher Donnell for all of their love. I am truly grateful to each of them for the encouragement, over many years, to complete this.

And certainly not least, to my husband Matthew Hagen for constant support and for giving me the time and space I needed to accomplish this. And to my daughters Solja Hagen and Lena Hagen who bring me joy every single day. 


\section{Table of Contents}

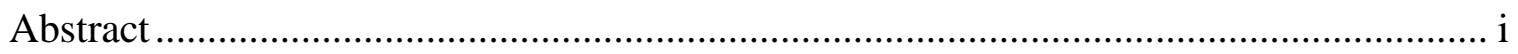

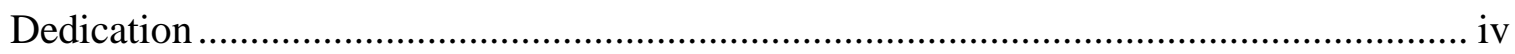

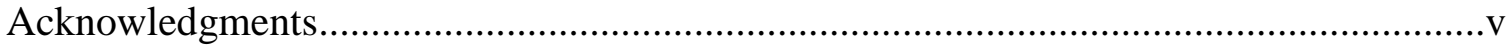

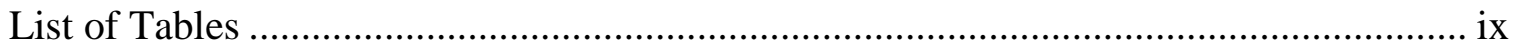

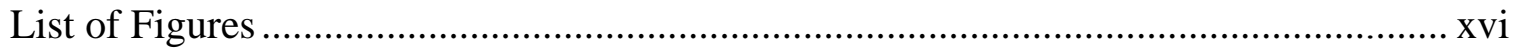

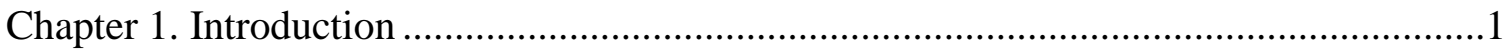

Transitioning to Value and the Role of Predictive Analytics ..............................1

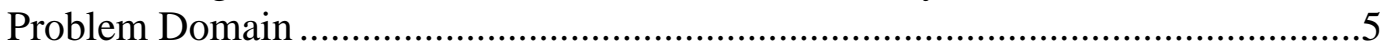

Research Objectives .........................................................................6

Preliminary Research Objective: LR and RA Comparison .......................6

Do RA and LR give the same results for equivalent models? ................................................................................6

Does exploratory RA provide better or novel models compared to LR? ................................................................6

Main Research Objective: Find Predictive Models with RA.................... 7

What are the best RA models?.................................................. 7

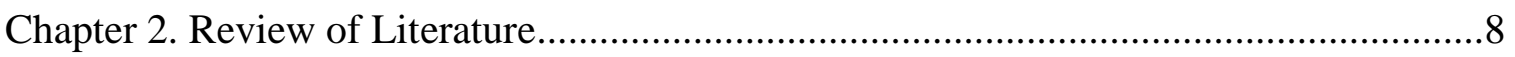

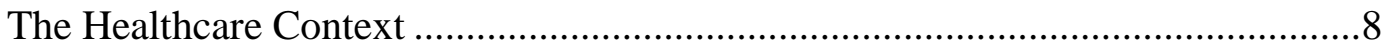

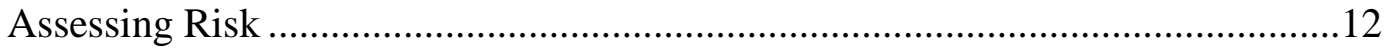

Risk Models and Methodologies in Healthcare ...............................................16

General Overview of LR and its Relation to RA ...............................................29

An Overview of RA ............................................................................... 30

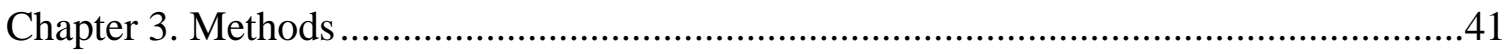

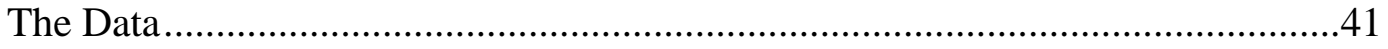

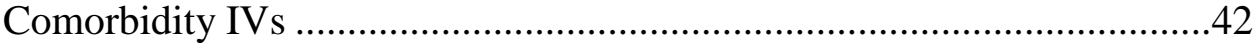

Non-Comorbidity IVs ...............................................................44

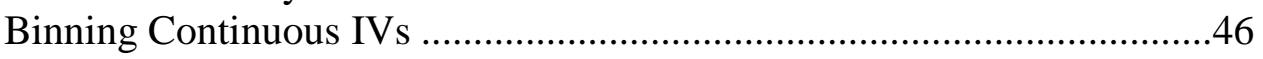

Dependent Variables (DVs) .........................................................4 47

Binning the Total Cost DV and Calculating Expected

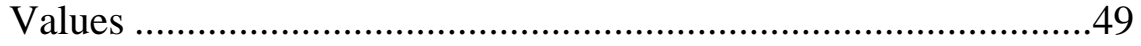

Associations between the DVs.................................................50

Formatting Files for Input into Occam Software ...................................51

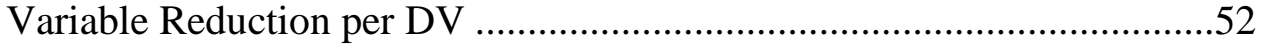

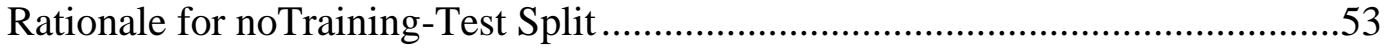

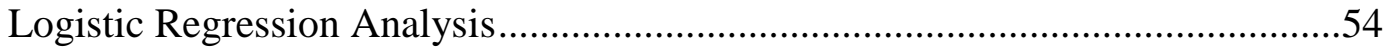




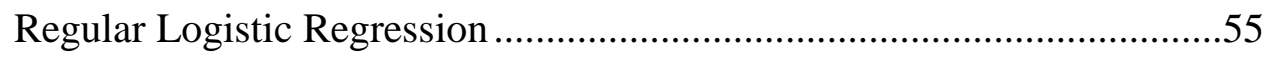

Stepwise Logistic Regression .............................................................56

Determining the Best RA Models .......................................................................57

Fitting the RA Models to the Data \& Identifying Important Model IV

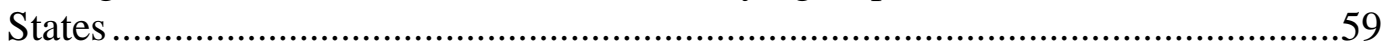

Selection Criteria for Important Model IV States......................................60

Calculating Risk Ratios..........................................................................61

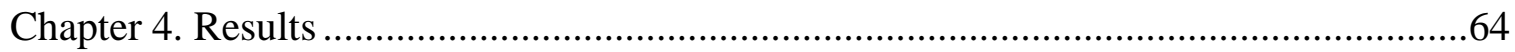

Preliminary Research Objective: LR \& RA Comparison .......................................64

Connecting to Previous LR Results from the Literature..............................64

Do RA and LR give the same results for equivalent models? ....................67

Does exploratory RA provide better or novel models compared to

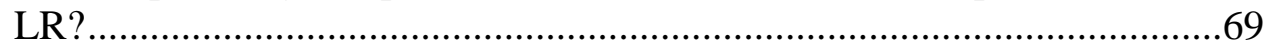

RA Exploratory and Stepwise LR....................................................................

Main Research Objective: Find predictive models with RA ………………….......76

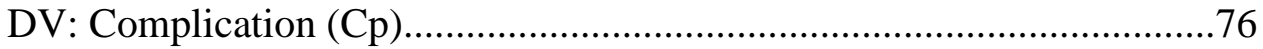

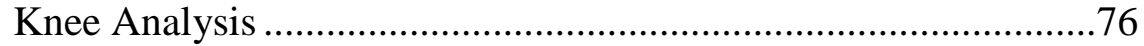

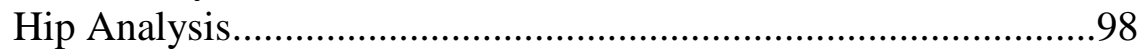

Hip \& Knee Summary of Results for Complication .....................112

DV: Skilled Nursing Facility (SNF) ...................................................112

Knee Analysis ...................................................................112

Hip Analysis...........................................................................125

Hip \& Knee Summary of Results for SNF ...................................135

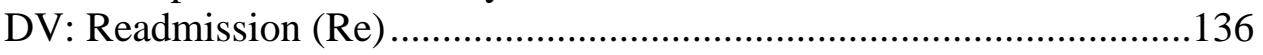

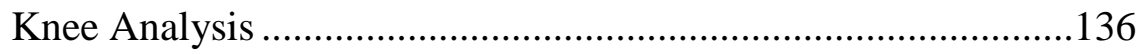

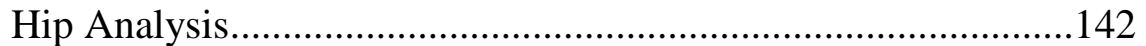

Hip \& Knee Summary for Readmissions......................................150

DV Total Cost (Tcb) ...................................................................150

Knee Analysis .......................................................................150

Hip Analysis..............................................................................162

Hip \& Knee Summary for Total Cost ..........................................172

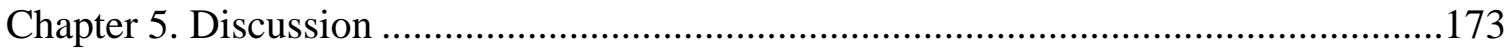

Clinical and Healthcare Related Contributions...................................................174

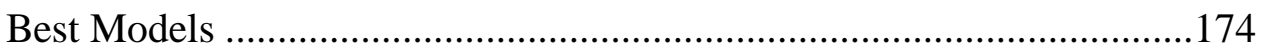

Important Predictors of Outcome Variables ............................................176

Patient Variables vs. Delivery System Variables .......................................180

Classifying Patients as Higher or Lower Risk for Adverse $\mathrm{Ou}$

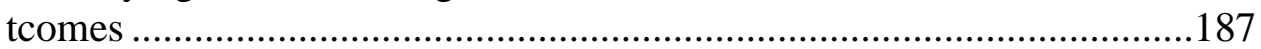

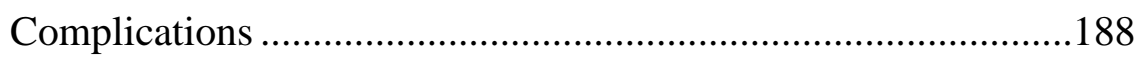

Discharge to skilled nursing facility ............................................190

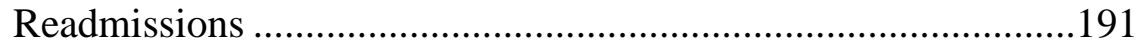

Total cost.........................................................................

Methodological Considerations .........................................................................193 


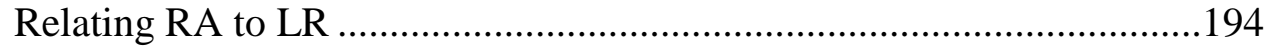

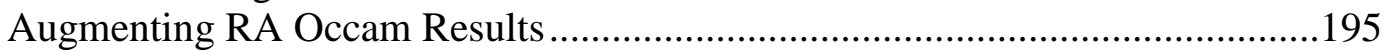

Tiered Classification System of Important Predicting Variables ............195

Decision Trees to Summarize Fit Results .............................................195

Generating Continuous Predictions ....................................................196

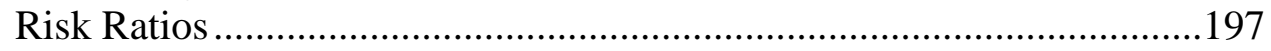

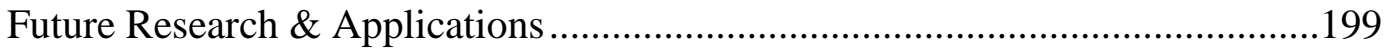

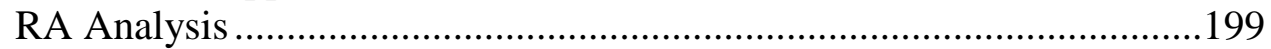

State-based Models ......................................................................199

K-systems and U-systems Analysis ...........................................200

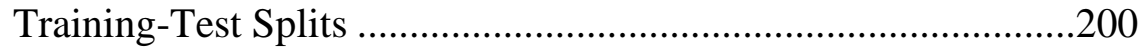

Binning 201

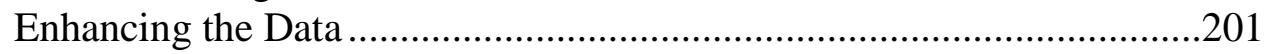

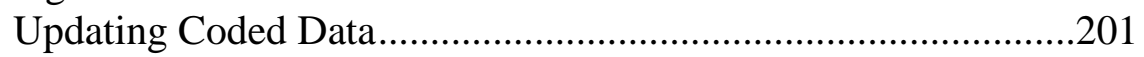

Adding Post-Discharge Data.....................................................202

Adding the Delivery System Data Domain ................................203

Reimbursement \& Allocation of Payments ............................................204

Translating Predictions into Value..........................................................205

Real-Time Risk Prediction...........................................................205

Measuring Quality \& Outcomes ...............................................207

Value Beyond Orthopedics .......................................................208

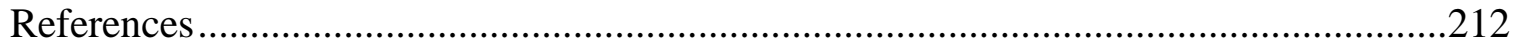

Appendix - Supplementary Tables..........................................................................222

Component Fit Tables for Knee SNF (All IVs) ...............................................229

Component Fit Tables for Knee (Comorbidity IVs Only) ....................................230

Component Fit Tables for Hip SNF (All IVs) ................................................231 


\section{List of Tables}

Table 1. Subset of Comorbidity IVs (Comorbidity IVs from Tier 1 Predictors)............... 44

Table 2. Subset of All IVs: Non-Comorbidity Variable Descriptions and IDs. .............. 45

Table 3. Binned IVs for the Continuous Variables: Number of Risks, Age, and Surgeon Volume. 46

Table 4. Description of the Original Dependent Variables and Subsequent Transformed (binned) Final Dependent Variables. ........................................... 47

Table 5. Binned IV (Tcb) for Total Cost Variable...................................................... 49

Table 6. Association of DVs by $\% \Delta \mathrm{H}$ in Knee Data Set .............................................. 50

Table 7. Association of DVs by $\% \Delta \mathrm{H}$ in Hip Data Set ............................................ 51

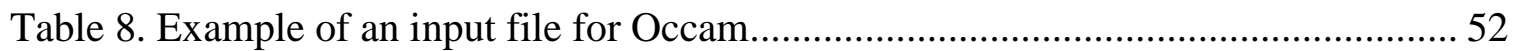

Table 9. Literature-based IVs Retained in all RA Exploratory Searches. ....................... 53

Table 10. Previous Results Reported in (Jain et al., 2005), and Results from New LR Analysis on this Project's Data. $\triangle \mathrm{df}, \triangle \mathrm{LR}$, and $\triangle \mathrm{AIC}$ values are given in the table for later comparisons with RA calculations. .................................... 66

Table 11. Previous Results Reported in (Jain et al., 2005) for 3 IVs, and Results from New LR Analysis on this Project's Data..................................................... 67

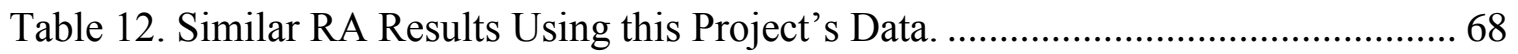

Table 13. Similar RA Results Using this Project's Data with 3 new IVs....................... 69

Table 14. Directed RA Search for DV Complication for 4 Variables. (Best Model by AIC in Bold.) ................................................................................. 71

Table 15. LR \& RA Comparison of Results for Single Predicting Search. ( $\Delta \mathrm{df}=1$ for every model in this table.) ............................................................................. 73

Table 16. Confirmatory LR Results for all 17 IVs and 6 IVs with $\mathrm{p}<0.05 . \ldots \ldots \ldots \ldots \ldots . . . . . . . .73$

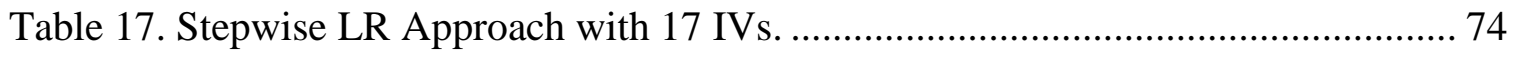

Table 18. RA Exploratory Search Results with 17 IVs (no interaction terms

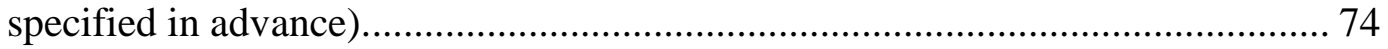

Table 19. LR Analysis with the 10 IVs present in the Best RA Model.......................... 75

Table 20. Summary of Search Results (Knee) for All IVs. Search covers coarse

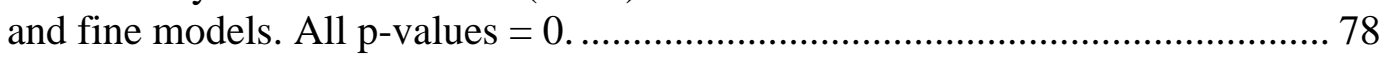

Table 21. IV Rku Predicts Rrd (as DV), Demonstrating IV Overlap............................. 80

Table 22. Summary of Search Results (Knee) for Comorbidity IVs. Search covers directed coarse and fine models. 
Table 23. The 3-Tiered Classification of Predicting Variables for DV:

Complication, Knee Analysis 84

Table 24. Full Fit Table (Knee) All IVs for Best Model: Ageb Cp : Nrb Cp : Ruh

$\mathrm{Cp}:$ Rhd Cp : Rku Cp : Rro Cp. Blue rows indicate ratio < 0.90 and orange rows indicate ratio $>1.10$. (Ratios between 0.91 and 1.09 are close to the margins and are indicated in gray.) 86

Table 25. Number of Risks (Nrb) Bin Range \& Frequency (Knee) 87

Table 26. Age Binned (Ageb) Bin Range \& Frequency (Knee). 88

Table 27. Component Fit Table for IVAgeb in (Knee) All IVs Best Model: Ageb Cp : Nrb Cp : Ruh Cp : Rhd Cp : Rku Cp : Rro Cp. Blue rows indicate ratio $<0.90$ and orange rows indicate ratio $>1.10$. (Ratios between 0.91 and 1.09 are close to the margins and are excluded.)

Table 28. Component Fit Table for IV Nrb in (Knee) All IVs Best Model: Ageb Cp : Nrb Cp : Ruh Cp : Rhd Cp : Rku Cp : Rro Cp. Blue rows indicate ratio $<0.90$ and orange rows indicate ratio $>1.10$. (Ratios between 0.91 and 1.09 are close to the margins and are excluded.)

Table 29. Condensed Fit Table of Comorbidity Components for IVs Ruh, Rhd, Rku, Rro in (Knee) All IVs Best Model: Ageb Cp : Nrb Cp : Ruh Cp : Rhd $\mathrm{Cp}$ : Rku Cp : Rro Cp. Blue rows indicate ratio $<0.90$ and orange rows indicate ratio $>1.10$. (Ratios between 0.91 and 1.09 are close to the margins and are excluded.)

Table 30. Fit Table (Knee) All IVs for Best Coarse Model: Nrb Rku Cp. Blue rows indicate ratio $<0.90$ and orange rows indicate ratio $>1.10$. (IV states with frequency $<10$ are not highlighted.)

Table 31. Fit Table (Knee) with Comorbidity IVs for best model Ruh Raf Cp : Ros Cp : Rhd Cp : Rav Cp : Reg Cp : Rku Cp : Ruu Cp : Rro Cp. Blue rows indicate ratio $<0.90$ and orange rows indicate ratio $>1.10$. 96

Table 32. Component Fit Table for IVs Ruh Raf (Knee) Comorbidity IVs Best Model Ruh Raf Cp : Ros Cp : Rhd Cp : Rav Cp : Reg Cp : Rku Cp : Ruu $\mathrm{Cp}$ : Rro Cp. Blue rows indicate ratio $<0.90$ and orange rows indicate ratio $>1.10$.

Table 33. Condensed Component Fit Table for IVs Ruh Raf (Knee) Comorbidity IVs Best Model Ruh Raf Cp : Ros Cp : Rhd Cp : Rav Cp : Reg Cp : Rku $\mathrm{Cp}$ : Ruu Cp : Rro Cp. Orange rows indicate ratio > 1.10. (Ratios between 0.91 and 1.09 are close to the margins and are excluded.)

Table 34. Summary of Search Results for All IVs for DV Complication (Hip). Search covers directed coarse, and fine models. All $\mathrm{p}$ values $=0$.

Table 35. Summary of Search Results for Comorbidity IVs (Hip). Search covers directed coarse, and fine models. 101 
Table 36. The 3-Tiered Classification of Predicting Variables for DV:

Complication, Hip Analysis.

Table 37. Fit Table (Hip) for All IVs for Best Model Ageb Cp : Nrb Cp : Rrd Cp :

Rca Cp : Rhd Cp : Rpl Cp. Blue rows indicate ratio $<0.90$ and orange rows indicate ratio $>1.10$. (Ratios between 0.91 and 1.09 are close to the margins and are excluded.) 105

Table 38. Number of Risks Binned (Nrb) Bin Range \& Frequency (Hip).................... 106

Table 39. Age Binned (Ageb) Bin Range \& Frequency (Hip). ................................... 106

Table 40. Component Fit Table for IVAgeb in (Hip) All IVs Best Model: Ageb Cp : Nrb Cp : Rrd Cp : Rca Cp : Rhd Cp : Rpl Cp. Blue rows indicate ratio < 0.90 and orange rows indicate ratio $>1.10$.

Table 41. Component Fit Table for IV Nrb in (Hip) All IVs Best Model: Ageb Cp : Nrb Cp : Rrd Cp : Rca Cp : Rhd Cp : Rpl Cp. Blue rows indicate ratio < 0.90 and orange rows indicate ratio $>1.10$.

Table 42. Condensed Component Fit Table for IVs Rhd, Rrd, Rpl and Rca in (Hip) All IVs Best Model: Ageb Cp : Nrb Cp : Rrd Cp : Rca Cp : Rhd Cp : $\mathrm{Rpl} \mathrm{Cp.} \mathrm{Blue} \mathrm{rows} \mathrm{indicate} \mathrm{ratio}<0.90$ and orange rows indicate ratio $>$ 1.10. (Ratios between 0.91 and 1.09 are close to the margins and are excluded.)

Table 43. Fit Table (Hip) for Comorbidity IVs for Best Model Rdi Cp : Ruh Cp : Rhh Cp : Ram Cp : Rra Cp : Rhe Cp : Rrd Cp : Rca Cp : Rhd Cp : Rpl Cp $: \operatorname{Rgp} \mathrm{Cp}: \mathrm{Rfr} \mathrm{Cp}$. Blue rows indicate ratio $<0.90$ and orange rows indicate ratio $>1.10$. (Ratios between 0.91 and 1.09 are close to the margins and are excluded.) (Variables Rhh, Ram, Rra, Rhd, Rgp, and Rfr only take value 0 for these rows.)

Table 44. Condensed Component Fit Table for IVs Rhd, Ram, Rhh, Rgp, Rfr, Rra, Rrd, and Rpl in (Hip) Comorbidity IVs Best Model: Rdi Cp : Ruh Cp : Rhh Cp : Ram Cp : Rra Cp : Rhe Cp : Rrd Cp : Rca Cp : Rhd Cp : Rpl $\mathrm{Cp}: \mathrm{Rgp} \mathrm{Cp}: \mathrm{Rfr} \mathrm{Cp}$. Orange rows indicate ratio $>1.10$. (Ratios between 0.91 and 1.09 are close to the margins and are excluded.)

Table 45. Summary of Most Important IVs by Tier across Hip and Knee for Complication.

Table 46. Summary of Search Results for Knee data, DV SNF. Includes both All $I V s$ for coarse and fine models.

Table 47. Summary of Search Results (Knee) for Comorbidity IVs for SNF.

Search covers directed coarse, and fine models.

Table 48. The 3-Tiered Classification of Predicting Variables for DV SNF, Knee Analysis.

Table 49. Fit Table (Knee) for All IVs for Best Model L SNF : Fc SNF : Ageb SNF : Svb SNF : Nrb SNF : Rmd SNF : Rug SNF : Rhf SNF. Blue rows 
indicate ratio $<0.90$ and orange rows indicate ratio $>1.10$. (Ratios

between 0.91 and 1.09 are close to the margins and are excluded.) 120

Table 50. Age bin distribution.

121

Table 51. Component Fit Table for IV L in (Knee) All IVs Best Model L SNF : Fc SNF : Ageb SNF : Svb SNF : Nrb SNF : Rmd SNF : Rug SNF : Rhf SNF.. Blue rows indicate ratio $<0.90$ and orange rows indicate ratio $>1.10$. (Ratios between 0.91 and 1.09 are close to the margins and are excluded.) ...... 122

Table 52. Component Fit Table for IV Fc in (Knee) All IVs Best Model L SNF : Fc SNF : Ageb SNF : Svb SNF : Nrb SNF : Rmd SNF : Rug SNF : Rhf $\mathrm{SNF}$. Blue rows indicate ratio $<0.90$ and orange rows indicate ratio $>$ 1.10.

Table 53. Fit Table (Knee) for Comorbidity IVs, for Best Model, Rhy SNF : Rau

SNF : Rmd SNF : Rpa SNF : Rug SNF : Rhe SNF : Rhf SNF : Rku SNF :

Rbn SNF : Rin SNF. Blue rows indicate ratio $<0.90$ and orange rows indicate ratio $>1.10$. (Ratios between 0.91 and 1.09 are close to the margins and are indicated in gray.).

Table 54. Summary of Search Results (Hip) for All IVs for for SNF. Search covers coarse and fine models

Table 55. Summary of Search Results (Hip) for Comorbidity IVs for SNF. Search covers both coarse and fine models.

Table 56. The 3-Tiered Classification of Predicting Variables for DV SNF, Hip Analysis

Table 57. Fit Table (Hip) for All IVs for Best Model Fc SNF : Ageb SNF : Svb SNF : Nrb SNF. Blue rows indicate ratio $<0.90$ and orange rows indicate ratio $>1.10$. (Ratios between 0.91 and 1.09 are close to the margins and are excluded.)

Table 58. Fit Table (Hip) for Comorbidity IVs for Best Model Rhy SNF : Rhh SNF : Rug SNF : Rhe SNF : Rrd SNF : Rav Rbn SNF : Rao SNF : Rse SNF : Rnr SNF . Blue rows indicate ratio $<0.90$ and orange rows indicate ratio $>1.10$. (Ratios between 0.91 and 1.09 are close to the margins and are excluded.) 134

Table 59. Summary of Most Important IVs by Tier across Hip and Knee for SNF...... 135

Table 60. Summary of Search Results (Knee) for All IVs for Readmission (Re).

Search covers coarse and fine models.

Table 61. Summary of Search Results (Knee) for Comorbidity IVs for DV

Readmission $(\mathrm{Re})$. Search covers coarse and fine models.

Table 62. The 3-Tiered Classification of Predicting Variables for DV: Re, Knee Analysis

$$
\text { - xii - }
$$


Table 63. Full Fit Table (Knee) for both All IVs search and Comorbidity IVs search for Best Model: Rci Re : Rco Re : Rgp Re : Rcj Re. Blue rows indicate ratio $<0.90$ and orange rows indicate ratio $>1.10$. (Ratios between 0.91 and 1.09 are close to the margins and are indicated in gray.)...... 141

Table 64. Summary of Search Results (Hip) for All IVs for DV Readmit (Re). Search covers directed coarse, and fine models.

Table 65. Summary of Search Results (Hip) for Comorbidity IVs. Search covers directed coarse, and fine models.

Table 66. The 3-Tiered Classification of Predicting Variables for DV: Readmission, Hip Analysis.

Table 67. Fit Table (Hip) for All IVs for Best Model Rhp Re : Rdd Re : Rpa Re :

Rep Re : Rhe Re : Rys Re : Rki Re. Blue rows indicate ratio $<0.90$ and orange rows indicate ratio $>1.10$. (Frequencies $<10$ and ratios between 0.91 and 1.09 are close to the margins and are excluded.)

Table 68. Condensed Fit Table of Components (Hip) All IVs Best Model Rhp Re :

Rdd Re : Rpa Re : Rep Re : Rhe Re : Rys Re : Rki Re. Orange rows indicate ratio $>1.10$. (Frequencies $<10$ are indicated in gray).

Table 69. Fit Table (Hip) for Comorbidity IVs for Best Model Rdd Re : Rpa Re :

Rep Re : Rhe Re : Rys Re : Rer Re : Rki Re Blue rows indicate ratio < 0.90 and orange rows indicate ratio $>1.10$.

Table 70. Condensed Fit Table of Components (Hip) All IVs Best AIC Model Rdd $\operatorname{Re}: \operatorname{Rpa} \operatorname{Re}: \operatorname{Rep} \operatorname{Re}:$ Rhe Re : Rys Re : Rer Re : Rki Re Re. Orange rows indicate ratio $>1.10$. (Frequencies $<10$ are indicated in gray).

Table 71. Summary of Most Important IVs by Tier across Hip and Knee for Readmission.

Table 72. Summary of Search Results (Knee) for All IVs for Total Cost Binned (Tcb). Search covers coarse and fine models

Table 73. Summary of Search Results (Knee) for Comorbidity IVs for DV

Total Cost Binned (Tcb). Search covers coarse and fine models. All $\mathrm{p}$ values $=0$.

Table 74. The 3-Tiered Classification of Predicting Variables for DV: Total Cost, Knee Analysis

Table 75. Bin values for DV Total Cost. 155

Table 76. Full Fit Table (Knee) All IVs for Best Model: S Tcb : Nrb Tcb. Blue rows indicate ratio $<0.90$ and orange rows indicate ratio $>1.10$ (ratios between 0.91 and 1.09 are close to the margins and are indicated in gray) 
Table 77. Component Fit Table for IV S (Knee) All IVs Best Model: L Tcb : Svb

Tcb : Nrb Tcb. Blue rows indicate ratio $<0.90$ and orange rows indicate ratio $>1.10$. (Ratios between 0.91 and 1.09 are close to the margins and are indicated in gray.) 158

Table 78. Component Fit Table for IV Nrb (Knee) All IVs Best Model: L Tcb : Svb Tcb : Nrb Tcb. (Ratios between 0.91 and 1.09 are close to the margins and are indicated in gray.)

Table 79. Full Fit Table (Knee) with Comorbidity IVs for Best Model: Ruh Tcb : Rmo Tcb : Rde Tcb : Ros Tcb : Rhe Tcb. Blue rows indicate ratio < 0.90 and orange rows indicate ratio $>1.10$ (ratios between 0.91 and 1.09 are close to the margins and are indicated in gray)....

Table 80. Summary of Search Results (Hip) for All IVs for Total Cost Binned $(\mathrm{Tcb})$. Search covers directed coarse, and fine models. All $\mathrm{p}$ values $=0$. 162

Table 81. Summary of Search Results (Hip) for Comorbidity IVs for DV Total Cost Binned (Tcb). Search covers coarse and fine models. All p values = 0 .

Table 82. The 3-Tiered Classification of Predicting Variables for DV: Total Cost, Hip Analysis. 166

Table 83. Total Cost Range, Average Cost, and Frequency. 167

Table 84. Full Fit Table (Hip) with All IVs for Best Model: L Tcb : Svb Tcb : Nrb Tcb. Blue rows indicate ratio $<0.90$ and orange rows indicate ratio $>1.10$. (Ratios between 0.91 and 1.09 are close to the margins and are indicated in gray.) 168

Table 85. Component Fit Table for IV L (Hip) All IVs Best Model: L Tcb : Svb Tcb : Nrb Tcb. Blue rows indicate ratio $<0.90$ and orange rows indicate ratio $>1.10$. (Ratios between 0.91 and 1.09 are close to the margins and are indicated in gray.)

Table 86. Component Fit Table for IV Svb (Hip) All IVs Best Model: L Tcb : Svb Tcb : Nrb Tcb. (Ratios between 0.91 and 1.09 are close to the margins and are indicated in gray.)

Table 87. Component Fit Table for IV Nrb (Hip) All IVs Best Model: L Tcb : Svb Tcb : Nrb Tcb. (Ratios between 0.91 and 1.09 are close to the margins and are indicated in gray.)

Table 88. Full Fit Table (Hip) with Comorbidity IVs for Best Model: Rmo Tcb : Rcp Tcb : Rrd Tcb : Rnr Tcb. Blue rows indicate ratio $<0.90$ and orange rows indicate ratio $>1.10$. (Ratios between 0.91 and 1.09 are close to the margins and are indicated in gray.)....

Table 89. Component Fit Table (Hip) for morbid obesity (Rmo) with Comorbidity IVs for Best Model: Rmo Tcb : Rcp Tcb : Rrd Tcb : Rnr Tcb. Blue rows

$$
\text { - xiv - }
$$


indicate ratio $<0.90$ and orange rows indicate ratio $>1.10$. (Ratios

between 0.91 and 1.09 are close to the margins and are indicated in gray.)...... 171

Table 90. Component Fit Table (Hip) for chronic pain (Rcp) with Comorbidity

IVs for Best Model: Rmo Tcb : Rcp Tcb : Rrd Tcb : Rnr Tcb. Blue rows indicate ratio $<0.90$ and orange rows indicate ratio $>1.10$. (Ratios between 0.91 and 1.09 are close to the margins and are indicated in gray.)...... 171

Table 91. Summary of Most Important IVs by Tier across Hip and Knee for Total Cost.

Table 92. List of 16 Models sorted by $\% \Delta H$. 174

Table 93. Top Important IVs across Hip and Knee Data Sets, and across the 4 DVs. $(\mathrm{Cp}=$ Complication, SNF = Skilled Nursing Facility, Re = Readmit, and $\mathrm{Tcb}=$ Total Cost Binned.) Novel variables are indicated by $*$. The Shared column indicates IVs located in both hip and knee data sets.

Table 94. Delivery System vs. Patient Related Submodels Comparison of Reduction of Uncertainty for DV Complication (Cp).

Table 95. Delivery System vs. Patient Related Submodels Comparison of Reduction of Uncertainty for DV Skilled Nursing Facility (SNF).

Table 96. Delivery System vs. Patient Related Submodels Comparison of Reduction of Uncertainty for DV Readmission (Re).

Table 97. Delivery System vs. Patient Related Submodels Comparison of Reduction of Uncertainty for DV Total Cost (Tcb).

Table 98. Summary of Decreased and Increased IV States by DV (Cp = Complication, $\mathrm{SNF}=$ Skilled Nursing Facility, $\mathrm{Re}=$ Readmission, $\mathrm{Tcb}=$ Total Cost). 188

Table 99. Increased and Decreased Risk IV States for Complication (Cp).

Table 100. Increased and Decreased Risk IV States for Skilled Nursing Facility (SNF)

Table 101. Increased and Decreased Risk IV States for Readmission (Re).

Table 102. Increased and Decreased Risk IV States for Total Cost (Tcb). 193 


\section{List of Figures}

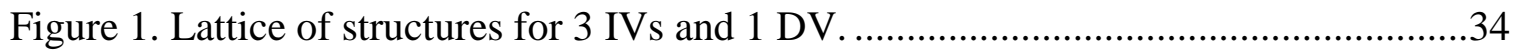

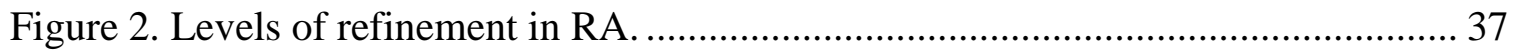

Figure 3. Knowing the Season Reduces the Uncertainty of the No-Rain:Rain from

$1: 1$ to $2: 1$ or $1: 2$ odds.

Figure 4. Decision Tree illustrating how knowing the Season Reduces the Uncertainty of the No-Rain:Rain from 1:1 to $2: 1$ or 1:2 odds.

Figure 5. Simplified Decision Tree for DV Any Comp (Knee) All IVs, for Best Coarse Model Nrb Rku Cp.

Figure 6. Decision Tree for DV Any Comp (Knee) All IVs for Best Model Ageb

Cp : Nrb Cp : Ruh Cp : Rhd Cp : Rku Cp : Rro Cp.

Figure 7. Decision Tree for DV Any Comp (Knee) with Comorbidity IVs for Best Model Ruh Raf Cp : Ros Cp : Rhd Cp : Rav Cp : Reg Cp : Rku Cp : Ruu Cp : Rro Cp.

Figure 8. Decision Tree for DV Any Comp (Hip) All IVs for Best Model Ageb Cp

: Nrb Cp : Rrd Cp : Rca Cp : Rhd Cp : Rpl Cp. 108

Figure 9. Decision Tree for DV Any Comp (Hip) with Comorbidity IVs for Best Model Rdi Cp : Ruh Cp : Rhh Cp : Ram Cp : Rra Cp : Rhe Cp : Rrd Cp : Rca Cp : Rhd Cp : Rpl Cp : Rgp Cp : Rfr Cp.

Figure 10. Decision Tree for DV SNF (Knee) with Comorbidity IVs for Best Model Rhy SNF : Rau SNF : Rmd SNF : Rpa SNF : Rug SNF : Rhe SNF : Rhf SNF : Rku SNF : Rbn SNF : Rin SNF.

Figure 11. Decision Tree for DV SNF (Hip) with Comorbidity IVs, for Best Model, Rhy SNF : Rhh SNF : Rug SNF :Rhe SNF : Rrd SNF : Rav Rbn SNF : Rao SNF : Rse SNF : Rnr SNF.

Figure 12. Decision Tree for DV Readmit (Knee) for Best Model Rci Re : Rco Re

: Rgp Re : Rcj Re.

Figure 13. Decision Tree for DV Readmit (Hip) All IVs for Best AIC Model Rhp $\operatorname{Re}: \operatorname{Rdd} \operatorname{Re}: \operatorname{Rpa} \operatorname{Re}: \operatorname{Rep} \operatorname{Re}:$ Rhe Re : Rys Re : Rki Re. 148

Figure 14. Decision Tree for DV Readmit (Hip) with Comorbidity IVs for Best AIC Model Rdd Re : Rpa Re : Rep Re : Rhe Re : Rys Re : Rer Re : Rki Re Re.

Figure 15. Decision Tree for DV Total Cost (Knee) with Comorbidity IVs for Best Model Ruh Tcb : Rmo Tcb : Rde Tcb : Ros Tcb : Rhe Tcb.

Figure 16. Decision Tree for DV Total Cost (Hip) with Comorbidity IVs for Best

Model Rmo Tcb : Rcp Tcb : Rrd Tcb : Rnr Tcb.

Figure 17. New Partition of IVs into Delivery System and Patient-Related IVs.

$$
\text { - Xvi - }
$$




\section{Chapter 1. Introduction}

\section{Transitioning to Value and the Role of Predictive Analytics}

Healthcare in the United States is facing unprecedented challenges. The trajectory of spending in US healthcare costs is unsustainable. This has led to a national dialogue that is currently under way among the federal government, insurance payers, healthcare delivery systems, and patient advocates — all calling for substantial changes to the current system in order to improve the value of healthcare.

One example where this challenge is clearly evident is in the field of orthopedics. The population in the United States that is age 65 and older is more active than in previous generations and expects to maintain a quality of life dependent on mobility. The demand for total joint replacement procedures is increasing. At the same time, Federal and state funding of Medicare and Medicaid services is in decline, and reimbursement rates are decreasing for these procedures.

The Patient Protection and Affordable Care Act of 2010 has already resulted in significant changes in how Medicare pays providers. These payment reforms are designed to slow the growth in costs and push improvements in quality of healthcare delivery. The Centers for Medicare and Medicaid Services (CMS) has already implemented a mandatory bundled payment for hip and knee replacements, with other condition groups soon to follow. In 2015, the Medicare Access and CHIP Reauthorization Act (MACRA) was passed, which changes the way CMS pays physicians for Medicare covered patients and includes programs such as The Merit-based Incentive Payment System (MIPS) and Advanced Alternative Payment Models (APMs), 
specifically tying payments to quality. In the last decade, the activation of healthcare reform is increasingly reflected in the policies and practices of both government and private health insurance payers and healthcare providers.

Bundled payments, and other new models of reimbursement, are now charged with finding ways to assess physician performance and create payment systems that determine what physicians should be paid for what they actually do. These new payment models will simultaneously include increased financial risk for health systems, insist on better quality outcomes, and demand lower cost. Under traditional payment models, healthcare providers were paid per service provided, and the outcome of that service was not linked to payment. Under new payment models, payment is increasingly tied to performance based on outcomes.

In fact, the dialogue among healthcare reform players has now focused on achieving value as the overarching goal of healthcare delivery. Value in healthcare has come to be specifically defined as the health outcomes achieved per dollar spent. The formula for value therefore places outcomes as the numerator and dollars spent as the denominator. A key driver of value is the aggregate set of services provided to a patient throughout an episode of care. The more focused and appropriate the services provided, given the unique patient factors, the more value can be achieved (Porter, 2010).

In President Barack Obama's 2015 State of the Union address, the President announced the Precision Medicine initiative, saying, "I want the country that eliminated polio and mapped the human genome to lead a new era of medicine — one that delivers the right treatment at the right time" (State of the Union Address, 2015). While precision medicine most frequently applies to the use of genomic level data, the term precision 
delivery refers to the use of a patient's electronic health record data to predict risk and tailor care to improve value. To increase value while payments are diminishing and tied to individual outcome quality, healthcare must improve at predicting risks and outcomes, and matching the right services to the right patient when needed.

The time for precision delivery is now. With the advent of accountable care, the health care organizations that succeed will be those that deliver high value. Perhaps the most important step to improving value will be implementing clinical analytics in routine care. Organizations that adapt by integrating these tools may do better both clinically and financially going forward. (Parikh, Kakad, \& Bates, 2016).

Some healthcare delivery system front runners are currently deploying predictive analytics in order to improve efficiency by tailoring the delivery of services to the individual patient. Delivery systems can focus costly resources on the higher-risk patient groups if patient risk is assessed in real time at the point of care. One way to improve predictions of risk is through better modeling methods.

Historically, predictive methods used fall under the category of generalized linear modeling, and more specifically Regression Analysis. Regression Analysis methods, such as Logistic Regression, have been widely accepted as the default method of prediction and are still the predominant methodology in health outcomes research. More recently, machine learning and artificial intelligence are gaining popularity, particularly with realtime analytics and risk prediction. Logistic Regression is broadly understood and has high "explainability," thus allowing the clinicians clear insight into the mechanism of prediction. Artificial Intelligence (AI) methods, such as Neural Networks (NNs), may enhance predictions, but perhaps with a sacrifice of explainability. If a methodology is 
both more predictive than LR and explainable, then this methodology will be worth considering.

This project explores the possibility that there are other methods of predictive modeling that may be stronger than or at least complimentary to standard methods of prediction. Reconstructability Analysis (RA), which is the methodology that this study looks at, is a machine learning methodology developed in the systems science research community for finding relations in data, especially non-linear and higher ordinality relations. These relationships between the variables in the data can have high ordinality (involve many variables), and one need not impose any hypothetical relationship prior to RA. RA may perform as well as LR methods and may provide additional accuracy through detection of novel variables and interaction effects between independent variables. While LR can model interaction effects, in standard implementation of this method these interactions must be specified during model construction, usually based on empirical findings, and specific hypotheses of interaction tested. RA is truly exploratory in that no interactions need to be specified during model construction, but emerge through exploration.

Reconstructability Analysis (RA) is a validated data mining method and has been used with success in other fields. It is relatively unknown, however, in health outcomes research. RA assesses hyper-graph structures either using set theoretic (SRA) or information theoretic (IRA) modeling. This study will use the information theoretic form IRA, which utilizes information theory to measure the uncertainty (entropy) in the output (dependent) variable(s), as a function of the known input (independent) variable(s). 
The potential value of RA for the present study is threefold:

(1) RA can play a confirmatory role. If RA results in similar findings as those arrived at through use of more commonly accepted methodologies (LR), then this confirmation of results increases the credibility of the research findings of the standard methodologies.

(2) RA can be used as a hypothesis generator, detecting variables that were not known a priori as strong predictors and then modeled using more standard regression analysis methods, therefore providing a complimentary and supportive approach.

(3) Further, RA might predict with better accuracy than LR. If this result is found, then RA is valuable as a stand-alone method that can replace LR in the creation of predictive risk models.

\section{Problem Domain}

Reform efforts are looking at joint replacement procedures as an area ripe for improvement in costs and outcomes because of the high cost, the high variation in outcomes, and the increasing demand for these procedures. This project presents condition specific models to predict outcomes that are important in hip and knee replacement. RA is used to look for predicting variables (including interaction effects) from a large set of patient comorbidities and delivery system variables on the following four dependent variables: Complication (inpatient), discharge to Skilled Nursing Facility (SNF), Readmission (90 day), and the continuous variable Total Cost (expected values are calculated for cost). 


\section{Research Objectives}

Within the specifics of the domain of hip and knee replacement surgery, this project aims to demonstrate that RA models are able to predict outcomes and provide additional insights that improve healthcare value, comparable to and beyond the capabilities of LR.

\section{Preliminary Research Objective: LR and RA Comparison}

Do $R A$ and LR give the same results for equivalent models?

Prior to exploratory modeling with Reconstructability Analysis, the first task is to demonstrate the validity of the data used in this project and the RA methodology. RA is validated by testing a logistic regression (LR)-generated model arrived at by a past study, using LR on this project's data. If LR applied to this data approximately reproduces LR results reported in the literature, and if the results of RA applied to this data are similar to those from LR, then the RA and LR methods are comparable. Generating similar results using RA gives confidence in the use of RA for exploratory modeling.

Does exploratory RA provide better or novel models compared to LR?

It is possible that RA produces better models than models produced by LR. If RA generates stronger predictions, then RA is not only valuable as a method to confirm or augment LR, but as a stand-alone method that could replace LR in the creation of predictive risk models. 


\section{Main Research Objective: Find Predictive Models with RA}

What are the best RA models?

The primary results of this project are a set of 16 predictive models. Each model provides a conditional probability distribution of the possible outcomes of four measures (DVs), given a set of comorbidities and delivery system variables (IVs). The models look not only at the probability distribution of outcomes given a single IV, or of multiple IVs taken individually, but also at the probability of outcomes given relationships between IVs, i.e., given complex interaction effects between the IVs and the DV.

The exploratory phase of this project aims to detect predictive IVs and interaction effects among the IVs and each of the DVs. Relations between the IVs and the DV do not have to be specified up front, and thus their form does not need to be known or hypothesized. These relations can be discovered using RA. These interaction effects may offer better prediction than that of single IVs known from the current literature. Exploratory modeling with RA may even detect surprising predictive IVs. 


\section{Chapter 2. Review of Literature}

In this chapter, four areas of literature are described. The first body of literature is that of the healthcare context, and new payment models spurred by healthcare reform efforts aimed at increasing value by tying costs to outcomes. The second area concerns the assessment of risk for quality measurement and optimization of real-time patient care. This discussion of risk is enhanced by the third area of focus, where the literature surveyed looks at specific predictive models in the analysis of outcomes and, although much less common, the clinical care setting. This literature shows that predictive models provide value, particularly condition/procedure-specific models. A large body of literature exists for predictive modeling in adult cardiac surgery, but a much more limited body of literature exists for hip and knee replacement surgery. Of the studies that exist in hip and knee replacement surgery, none make use of data mining techniques to increase predictive ability. These studies use regression analysis methods with the most common approach being the logistic regression methodology. That being said, in the fourth main body of literature, data mining techniques are reviewed and the potential role for Reconstructability Analysis to add value as a methodology is highlighted.

\section{The Healthcare Context}

Costs in the US healthcare system have spiraled out of control, resulting in what is referred to as a healthcare crisis. This crisis is reflected not only in soaring costs, but also in lack of access to care and in variation in treatments and outcomes and many other issues (Fisher, 2003). National Health Expenditure Projects Americans will spend 4.5 trillion dollars on healthcare by 2019 (Medicare, 2016). The US is an outlier in 
healthcare spending, spending $40 \%$ more per capita then the next highest spending country (Lorenzoni, Belloni, \& Sassi, 2014). This massive amount of spending has not resulted in better care. Glaring variations in services provided and outcomes have intensified efforts toward reforming the healthcare delivery system. In a system that has traditionally paid a fee for every service provided, current policy reform is essentially focused on removing the incentive to provide too much care by creating fixed payments, and guarding against poor care by incentivizing the delivery system on good performance based on quality (Doyle, Graves, \& Gruber, 2015). In a seminal publication, Michael Porter captured the conflicting goals of stakeholders, including access to services, profitability, high quality, cost containment, safety, convenience, patient-centeredness, and satisfaction, and introduced the concept of value as the overarching goal to unite healthcare delivery (Porter, 2010). Arguing for value, Porter states "If value improves, patients, payers, providers, and suppliers can all benefit while the economic sustainability of the health care system increases" (Porter \& Teisberg, 2006).

Literature on healthcare reform shows the evolution toward value-based care over the last decade, through adoption of performance-based payment systems as a primary mechanism for the reduction of soaring healthcare costs and improvement in quality care (Nichols \& O'Malley, 2006). The Patient Protection and Affordable Care Act (ACA) of 2010 ("Patient Protection and Affordable Care Act of 2010," 2010) has been a significant piece of legislation with myriad components and strategies. A central piece of this legislation provides for slowing growth in Medicare spending and promotes experiments in payment and delivery system reform (Oberlander, 2010). Under value-based payment systems, the fee-for-service payment system is retained but tied to efficiency and quality 
by the delivery system. The transformation of healthcare is under way, with $30 \%$ of Medicare payments going through alternative payment models (APMs) (Obama, 2016). These APMs include bundled payments, where a single payment is reimbursed for all services falling within a pre-defined episode, or accountable care organizations (ACOs). According to CMS, an ACO is "an organization of health care practitioners that agrees to be accountable for the quality, cost, and overall care of Medicare beneficiaries who are enrolled in the traditional fee-for-service program who are assigned to it" (Centers for Medicare \& Medicaid Services (CMS), HHS, 2011).

One of the first specific clinical areas to face these reform efforts is hip and knee replacements. The demand for hip and knee replacements, coupled with the variation in cost and outcomes, has resulted in the first early bundled payment experiments, such as Bundled Payment for Care Improvement Initiative (BPCI) and now the first mandatory bundled payment for hip and knee replacement surgery by the Centers for Medicare and Medicaid Services (CMS). In the CMS Comprehensive Care for Joint Replacement (CJR) episode-based payment model, set target payments require hospitals, physicians, and post-acute care providers to coordinate in order to improve patient outcomes at an increasingly smaller target price (Centers for Medicare \& Medicaid Services, 2015). Additionally, a large number of joint replacement commercial bundled contracts are in the marketplace today.

Demand for total hip and knee replacement is expected to continue rapid growth in the next 10 years, largely due to the aging baby boomer population and the obesity epidemic (Fehring et al., 2010). One estimate places the total number of hip and knee replacements at over 4 million by 2030, an increase of $174 \%$ over 2007 volumes 
(Kurtz S, 2007). Estimates place the prevalence of osteoarthritis, the disease causing total hip and knee replacement, at $18.2 \%$ by 2020 (Lawrence et al., 1998). Osteoarthritis increases with age (Pulido L, 2008) and "the higher life expectancy and the upcoming massive cohort from the 'old baby boomers' will lead to a higher number of joint arthroplasties being performed ... it is hence plausible that a higher incidence of medical complications in this growing joint arthroplasty population will be observed" (p.139). The quality of elective total hip and total knee procedures is extremely varied (Tomek et al., 2012) and in fact, hip and knee replacements are among the procedures with the most varied payments. These variations in payment correspond in part to the fact that the profile of patient populations differ across regions and within regions by the fact that some hospitals receive larger burdens of higher-risk patients. Even after controlling for the differences between patient populations, there is still a large variation between payments for these procedures (Miller et al., 2011). The authors suggest that the unexplained remaining variation is possibly unwarranted, suggesting that there is room to improve. The literature shows a broad consensus that the medical system can perform better-providing good patient outcomes at a lower cost.

Delivery systems contracted under bundled payments will have to deal with the issue of risk: what happens if the cost of care exceeds the set bundled price? In some bundle arrangements, the physician is a partner with the hospital on the gains, and in some scenarios also on the loss. A set bundled price therefore provides incentive to come in under the set fixed price as often as over it and therefore break even, or better yet, come in under the bundled price more often than not and therefore derive profit. Reducing costs alone will not solve the healthcare crisis, and achieving high quality 
outcomes is an essential component of value. Porter states "Cost reduction without regard to the outcomes achieved is dangerous and self-defeating, leading to false "savings" and potentially limiting effective care." (Porter \& Teisberg, 2006). Alternative payment models reward cost effectiveness, and there is concern that without adjusting for patient comorbidities restricted access to care will be encouraged (Rozell, Courtney, Dattilo, Wu, \& Lee, 2016). An increase in demand for total hip and knee replacement surgery in a market where surgeons must select only a subset of all surgical candidates for surgery and a reimbursement model that does not account for patient risk is a recipe for higherrisk patients to be pushed out of the pool of surgical candidates.

\section{Assessing Risk}

Accounting for patient risk is important not only for optimizing the value equation and ensuring fair physician reimbursement, but for patients, ensuring that reimbursement is structured in a way that allows for broad and equitable patient access. With payments increasingly tied to outcomes, providers are demanding collection of better data on outcomes and improving risk adjustment techniques to account for underlying patient comorbidities and understanding what techniques lead to the best outcomes (Luft, 2009).

There are two primary ways that the assessment of risk plays a role in this landscape. First, adequately understanding a patient's risk allows for the measurement of outcomes adjusted by patient individual risk factors. This retrospective measure of quality is critical for the measurement of performance. Second, predicting risk can allow for-real time identification of high-risk patients likely to require expensive care or to 
experience an adverse event (Bates, Saria, Ohno-Machado, Shah, \& Escobar, 2014) as

well as low-risk patients who may be appropriate candidates for fast-track care pathways.

Whether the reimbursement is an APM bundled-payment model or not, there are challenges when tying payment to performance. In an article looking at pay-forperformance in orthopedics, authors describe these challenges:

The implementation of such a dramatic paradigm shift in healthcare payment policy is fraught with challenges. Those challenges include difficulty in defining and measuring quality and efficiency, cost of collecting and analyzing performance data, development and implementation of appropriate risk adjustment models, lack of additional funding to reward quality, unintended consequences of provider "gaming" and patient deselection (e.g., "cherry picking"), and impact on low-tier, low quality providers. (Bozic, Smith, \& Mauerhan, 2007)

There are several limitations that must be addressed before widespread implementation of new payment models that tie payment to quality. One of these limitations is inadequate risk adjustment for clinical outcome measures (Nichols \& O’Malley, 2006). The authors state that:

Inadequate information systems, as well as imperfect algorithms and data to control for patient-level comorbidities, severely limit the ability to risk adjust clinical outcomes measures. This is a major barrier to more widespread implementation of pay for performance (P4P) and to convincing some physicians, who manage complex patients on a daily basis, of the value of these measures. (Nichols \& O'Malley, 2006).

Risk adjustment models have been developed and implemented for the large-scale insurance market. This is the actuarial side of healthcare finance. Insurers have always understood that if they can attract healthier patients to their pool of enrollees then they will pay out less in medical claims. Health insurers have long used risk adjustment methods to group patients into risk cohorts for payment strategies. At present, the type of risk adjustment that occurs at the level of the health insurer is based on complex 
algorithms with the sole purpose of making adjustments between populations of patients for financial balance in the insurance market. This type of risk adjustment is evolving under the Affordable Care Act, where Health Insurance Exchanges are now mandated. This new model of risk adjustment will utilize retrospective, diagnosis-based risk adjustment strategies as a way to compensate insurers with higher-risk populations (Weiner, Trish, Abrams, \& Lemke, 2012). This large-scale risk adjustment performed in the insurance market is very different from the prospective risk models that will be created in this project. While the former is critical for a functional insurance market, it cannot be applied at the patient or physician level and cannot calculate an expected outcome.

In order to assess physician performance based on patient outcomes, it is crucial to determine if the observed outcome for a group of patients is better or worse than expected based on the patient comorbidities rather than better or worse than a fixed target based on an average. Payments based on average targets are frequently referred to as global payments. With or without physician performance incentives, the practice of global payment is worrisome. If an expected outcome is simply a fixed target, providers are punished "whose complex patients, even if doing 'better than expected,' do not hit targets that are easier to achieve with healthier patients" (Ash AS, 2012). Target outcomes that adjust based on the patients' risk are critical for assessing physician performance. Goroll and Schoenbaum (2012) describe robust, scientifically validated risk-adjustment models as critical in order to address the impediments to payment reform. Global payments, without risk adjustment, burden the providers with significant financial risk. Risk adjustment that accounts for performance based on expected outcomes versus 
an average-based target will help alleviate this financial risk and deter providers from the “temptation inherent in global payment to cherry-pick patients." Utilizing risk adjustment models at the provider level will enable patient-based expected outcomes to be generated, and can connect directly with pay-for-performance strategies. Goroll and Schoenbaum (2012) state that "Risk adjustment applied to payment for performance can serve to recognize, reward, and incentivize the extra work needed to achieve better-than-expected outcomes, helping to alleviate the concern that global payment will lead to less care." Ellis and Ash (2012) are also concerned about the use of globally calculated performance measures and the lack of methods to make patient-based adjustments stating that "Although using non-adjusted performance measures may create undesirable incentives for practices to avoid the sickest patients, even crude adjustments are rare."

A seminal report issued by the Institute of Medicine (IOM), an arm of the National Academy of Sciences titled "Crossing the Quality Chasm: A New Health System for the 21st Century," makes the following request:

The committee calls for all purchasers, both public and private, to carefully reexamine their payment policies to remove barriers that impede quality improvement and build in stronger incentives for quality enhancement. Clinicians should be adequately compensated for taking good care of all types of patients, neither gaining nor losing financially for caring for sicker patients or those with more complicated conditions. Payment methods also should provide an opportunity for providers to share in the benefits of quality improvement, provide an opportunity for consumers and purchasers to recognize quality differences in healthcare and direct their decisions accordingly, align financial incentives with the implementation of care processes based on best practices and the achievement of better patient outcomes, and enable providers to coordinate care for patients across settings and over time.

Achieving high-quality outcomes can certainly be enhanced with retrospective measurement, appropriately adjusted for patient risk. Quality improvement programs 
have been built using measures based on retrospective data. But the question remains: Can we do something to prevent rather than merely to adjust? If we can predict, we can prevent - or so the theory goes. With delivery systems accountable, predictive algorithms can help allocate resources more effectively for both high-risk and low-risk patients. Successful organizations will use a broad array of tools to predict important outcomes, including to identify patients likely to require expensive care, be readmitted, or experience a specific type of adverse event. (Bates, Saria, Ohno-Machado, Shah, \& Escobar, 2014).

\section{Risk Models and Methodologies in Healthcare}

In this section of the literature review, existing risk models are reviewed. Literature shows that for condition-specific hip and knee replacement models, most are based on logistic regression (LR) and previously identified comorbidities in a retrospective analysis. Additionally, literature is reviewed showing some promising implementations of real-time risk prediction systems, offering the chance to prevent costly care and poor outcomes.

Predicting risk of adverse outcomes is common practice in insurance and in research. Many insurers use risk assessment and risk adjustment models to identify patients who are at risk of high-cost care as well as to profile and rate physicians. Highrisk care management is used for patients identified as expected to incur high costs. However, broad risk profiling may not be suitable for subgroups of patients with specific clinical conditions and interventions. "Interventions that are appropriate and effective for one group will often do little to improve care and reduce costs for others" (Powers \& 
Chaguturu, 2016). Insurance companies have long been involved in risk adjustment, and have large pools of claims data for their members, yet are always on the lookout for better methodologies to improve predictions of expected utilization.

There are a handful of general risk adjustment models that are based on preoperative clinical characteristics such as: Adjusted Clinical Group (ACG), Diagnostic Cost Group (DCG), Seattle Index of Comorbidity (SIC), Chronic Illness and Disability Payment System (CDPS), Charlson Index, RxRisk, Self-reported measures for demographic and health, and prior year expenditures. In one study, Maciejewski (2005) and Liu compared the predictive accuracy of these risk adjustors for prospective modeling of the expenditures in a year period of time. The goal of this study was to look at which measures were most predictive and ought to be considered in future studies. One of the interesting results from this study was that administrative-based data performed better than patient self-reported measures. Administrative data refers to data that is most often collected by government or commercial payers, typically for reimbursement purposes.

Administrative data sets are large, inexpensive, structured, and readily available, however they lack important clinical information. As the authors state: "VA provides an ideal setting to assess the performance of differing strategies to adjust for patient risk differences in observational or experimental studies because of the availability of extensive demographic, clinical, pharmacy, and economic data on several million veterans who use VA services" (Maciejewski \& Liu, 2005). This type of model validation would be very difficult in hip and knee replacement, as there is little accessibility to comparable data. 
The private sector has recognized an unmet need for risk prediction, and a handful of proprietary risk adjustment models exist. Currently, there are general risk calculators available to providers marketed by private companies such as Archimedes, which markets the product IndiGO. In this case, as is true with virtually all privately marketed risk models, the mechanisms by which prediction is calculated are at least partially a "black box," meaning that the end user does not know exactly how a score was calculated. In IndiGO, patient data is entered into a software application, and after the data is entered, "IndiGO uses its advanced algorithms, based on the Archimedes Model, to create individualized guidelines. The individualized guidelines include person-specific risk of adverse events (such as heart attack, stroke, diabetes onset and its complications) ..." (Bellows, Patel, \& Young, 2014). The predictive value of these models can be-and has been — validated against compared with other models based simply on how well they predict. Not only are they comparable, but they can be included in other risk scoring models as well. For example, in the Global Outcomes Score (GO Score), Eddy, Adler \& Morris (2012) use the IndiGO risk calculator as the method to predict multiple outcomes across many healthcare domains. The GO Score looks at a spectrum of outcomes, comorbidities and interventions. The GO Score takes aggregated predicted outcomes and measures them against the observed outcomes. The GO Score demonstrates the use of predicted versus observed outcome as a method to quantify and measure quality. Quality occurs when a patient does better (observed outcome) than expected (predicted risk). The GO Score could be augmented, and utilize another risk calculator, as the authors suggest: "Other risk calculators can be used to generate GO Scores, but they should be similarly validated" (Eddy, Adler \& Morris, 2012). 
While global risk models may be validated and useful at a general level, models constructed for specific procedures are needed. Each surgical procure has its own patient population with different rates of comorbidities, and disease-specific risk models are more precise in predicting outcomes. For example, newborn infants will have a different set of comorbidities than elderly patients prior to heart surgery. Not only are the comorbidities different, but the heart surgeries conducted on each are technically unique, and the adverse outcomes related to the procedure are distinct.

The procedure-specific Aristotle Based Complexity (ABC) score is used to predict complications, mortality, and prolonged length of stay for surgeries performed by congenital cardiac surgeons (Jacobs JP, 2009). The ABC score classifies the procedure and assigns it a case complexity allowing placement of cases into low-risk or high-risk groups, thus enabling researchers to make adjustments based on risk in order to conduct outcomes analysis. The ABC score is uniquely created to apply to congenital heart surgery, performed almost entirely on infants and children, and has no widespread use outside of congenital heart surgery.

The most widespread and commonly used procedure-specific risk models are found in adult cardiac surgery outcomes research. The body of literature on predictive modeling is largely composed of studies aiming to predict operative mortality and complications after adult cardiac surgery. These studies often use predictive risk models created and maintained by the Society of Thoracic Surgeons (STS) and EuroScore. The plethora of studies may have resulted from the fact that the STS and EuroScore models are available online as a simple-to-use calculator, allowing widespread application. Head et al. (2013) performed a systematic review of risk prediction and located 5,768 
studies modeling outcomes in adult cardiac surgery. They ended up reviewing 844 studies identifying preoperative independent comorbidities for adverse outcomes after adult cardiac surgery. In this review, the authors found many instances of independent variables that were found to be predictive of outcomes, but many of these variables were not frequently considered in the bulk of the predictive studies. The authors conclude, "Risk estimates of mortality, stroke, renal failure and length of stay may be improved by the inclusion of additional (non-traditional) innovative comorbidities. Current and future databases should consider collecting these variables" (p. 121). What this study demonstrates is the need for exploratory modeling to reveal unexpected variables that may have predictive strength. These would be what the authors refer to as "innovative" or "non-traditional" variables.

Cagini (2012) discusses what may be considered the traditional variables popular in risk prediction in adult cardiac surgery, stating:

A core set of variables associated with outcomes in cardiothoracic surgery have evolved over time. Accuracy of risk models developed based on administrative data in New York and Pennsylvania have been shown to be substantially improved by addition of a few critical clinical variables. ... One may further question how many variables are actually needed to have a robust prediction model (p. 68).

This quote illustrates that commonly-used predictive variables are empirically grounded on past studies, yet accuracy in these studies was improved by adding new variables. This raises the question of which variables, exactly, should be added. The authors ask, "How many variables are actually needed to have a robust prediction model?" (p. 68). The RA exploratory modeling approach is a response to exactly this type of question. RA can search through even thousands of variables, and can detect the 
variables and their relations that are most predictive. However, exploratory results should be confirmed with confirmatory tests.

Cagini (2012) further points out the primary methods used in the thousands of adult cardiac outcomes studies. The author determines that logistic regression models are the most common method for risk modeling and site comparative studies, demonstrating that logistic regression models offer the best overall performance. The author discusses potential advances that may be offered by the use of machine-learning techniques "as these models permit complex, nonlinear information processing. However, tests of these models have not yet shown significant improvement over logistic [ . . ] models." Like RA, the neural networks methodology often falls under the more general class of methods referred to as machine learning. The literature shows a very limited testing on the ability of machine learning to predict better than logistic regression, with studies largely falling within the arena of adult cardiac outcomes research (Lippmann, Kukolich \& Shahian, 1995), (Tu JV, 1998). Lippmann and Shahian (Lippmann RP, 1997) compared a neural network model to logistic regression, finding that "a committee classifier combining the best neural network and logistic regression provided the best model calibration ..."-suggesting that there is possibly a combination of methods used to get best prediction.

Compared to the thousands of studies conducted in adult and congenital heart surgeries, studies conducted in the field of orthopedics are relatively few. Of the outcomes studies published in orthopedic journals, the majority look at the effect of traditional comorbidities on one or more adverse outcomes. Bjorgul, Novicoff \& Saleh (2010) discuss this body of literature: 
There is ample evidence that comorbidity is a major factor in determining the outcomes of various conditions, and there is a large body of literature discussing the multiple aspects of comorbidity. The general finding is a close relationship between comorbidities and complications, mortality, functional outcome, and consumption of healthcare resources (Bjorgul et al., 2010).

Comorbidities previously found to be predictive of adverse outcomes include obesity (Andrew et al., 2008; Suleiman et al., 2012), diabetes (Bolognesi et al., 2008; Berbari et al., 1998; Everhart, Altneu \& Calhoun, 2013), hypertension (Memtsoudis et al., 2010; Jafari, Huang, Joshi, Parvizi \& Hozack, 2010), and age (Polanczyk et al., 2001; Memtsoudis, González Della Valle, et al., 2010), heart failure, pulmonary issues (Bozic et al., 2012; Jain, Guller, Pietrobon, Bond \& Higgins, 2005) among others. Almost without exception, these studies used logistic regression methods to test a hypothesis that a relationship exists between a comorbidity and the outcome of interest. Studies that looked at more than one potential comorbidity used multivariable logistic regression techniques to see if there was an effect on the outcome from more than one comorbidity. This body of literature illustrates the current approach to performing retrospective risk prediction in hip and knee replacement, which is limited to using previously validated comorbidities in order to predict adverse outcomes. A good portion of the literature on outcomes in hip and knee replacement is not focused on adverse outcomes at all, but is focused on the functional and general patient-reported health from follow-up surveys administered postoperatively and over the years of follow-up visits (Caracciolo \& Giaquinto, 2005; Gandhi et al., 2010). These studies require data collection normally outside of the standard of care, are resource-intensive to collect, and require prognostic study design and close monitoring of patients for long-term follow-up. 
The broad category of adverse outcomes includes not only post-surgical complications, but outcomes such as discharge destination, readmissions, length of stay, and total cost. These endpoints can all be measured with commonly available administrative claims databases, a prerequisite for a simple and widely available risk prediction. After hip or knee surgery, a patient may be sent directly home after the hospital stay or may be discharged from the hospital directly to an extended care facility (ECF). Researchers have looked at the impact of certain comorbidities on the discharge destination of patients after hip and knee replacement surgery (Bozic, Wagie, Naessens, Berry, \& Rubash, 2006; Oldmeadow, McBurney, \& Robertson, 2003; Barsoum et al., 2010.) Munin et al., (1995) looked at the outcome of being discharged to an extended care facility (ECF) and found that patients discharged to an ECF had increased comorbidities prior to surgery. They developed a logistic regression model that was able to predict $76 \%$ of the discharges to an ECF. This high predictive ability included not only comorbidities, but operative and postoperative data as well. Using post-discharge data to calculate risk does not allow for prognostic risk calculation and makes unclear the extent to which the patient's risk played a role relative to the in-hospital processes of care and medical complications that patient may have experienced.

A prolonged length of stay (LOS) is another nonmedical indicator of adverse outcomes. Researchers have looked at the impact of certain comorbidities on length of stay of patients after hip and knee replacement surgery (Clague et al., 2002; Md, Elsharkawy, 2011; Cram, 2011; Polanczyk et al., 2001). Dall et al., (2009) looked at the association between various comorbidities and LOS using standard logistic regression techniques. Understanding the need for a simple and easily available calculator, the 
authors discussed the possibility that findings from their research will contribute to the creation of a simple scoring system to predict LOS. This study discussed the need to have this available as a prognostic tool in order to increase patient flow in the hospital and adjust staffing levels based on case mix (Dall et al., 2009). Interestingly, this multivariate study found that the day of the week and the surgeon performing the surgery had an effect on LOS. Because this information is routinely collected and is easily available in claims data, future model development should include these variables alongside the patient's medical comorbidities. Other research has confirmed surgical volume in a given hospital as predictive of complications after joint replacement (Schroer, 2008).

A key opportunity for delivery systems to improve value is by limiting overuse of costly resources by focusing these resources on high-risk patient groups (Bates et al., 2014). Screening patients for relevant comorbidities prior to surgery allows for increased medical interventions, which have proven to be successful in decreasing adverse outcomes (Meding, 2007). "Estimating the risk of complications when a patient first presents to a hospital can be useful for a number of reasons, such as managing staffing and bed resources, anticipating the need for a transfer to the appropriate unit, and informing overall strategy for managing the patient" (Bates et al., 2014). Radcliff et al. (2012) discuss the success of preoperative risk stratification on reducing cardiac and thromboembolic complications in elective hip and knee replacement surgery, but point out that few studies have been done on non-cardiac medical complications. The authors subsequently present a risk stratification tool constructed from comorbidities confirmed in previous studies and physician interviews. This study went beyond a demonstration of a relationship between comorbidities and non-cardiac complications, but provided a 
method to increase and measure quality. The researchers in this study compared the observed outcomes of patients who were screened against the observed outcomes patients who were not screened (Radcliff et al., 2012). If the screened group has better outcomes than the group that was not screened, then the screening tool is shown to have a positive effect on quality.

Oldmeadow et al. (2003) developed The Risk Assessment and Predictor Tool (RAPT) as a way to simply and easily predict a patient's risk of requiring discharge to an extended care facility (ECF). Initially constructed from a logistic regression model, it was subsequently transformed into a simple points-based scoring system that was validated on patient populations in its development and applications. The RAPT score places patients into one of three risk groups. This score and the subsequent risk group the patient is placed in are designed specifically to provide an objective measure of who ought to be placed on a home pathway (those with a RAPT score >9) and those who need definite rehabilitative care (those with a RAPT score <6) (Dauty, Schmitt, Menu, Rousseau \& Dubois, 2012).

A risk calculator developed for total joint arthroplasty predicting discharge to skilled nursing facility (SNF) using logistic regression found age, dependent functional status, living location, and severity of illness (ASA score) to be predictive of SNF discharge (Gholson et al., 2016). However, it is quite well known that the utilization of SNF varies by surgeon and hospital location, but no specific studies look at the relative predictive effects of patient comorbidities versus provider practice patterns. This research project will look at SNF, and test the effects of patient-related versus delivery system predictors. 
Romine, May, Taylor \& Chimento (2013) created a total knee replacement risk calculator and found it to have predictive value of perioperative complications. This evaluation resulted in 19 comorbidities as predictive of " 1 or more" complications modeled using logistic regression. The inputs for the calculator include the patient's comorbidities, which are necessary for prognostic prediction and can support patient care decisions. While Romine et al.'s calculator predicts perioperative complications, including complications that occur in the hospital prior to discharge, it also includes complications that occur within 14 postoperative days. This post-discharge data is problematic in its inherent incompleteness since post-discharge data is available for only a fraction of the total population of the surgical cases. Another limitation of this calculator is that it was built using Medicare data, and then tested on a more diverse population of patients, resulting in an overestimate of complications due to the known fact that the Medicare population has a higher incidence of complications (Romine et al., 2013). Because Medicare datasets are large, well validated, accessible, and comparable to other studies using the same data, Medicare data is commonly used. The difficulty in more widely applying a model derived from Medicare data points to the need to construct a model on data that includes the non-Medicare commercial patient mix as well. That being said, this risk calculator fills a void in the field of health outcomes research and is considered by Romine et al. (2013) to be:

the first risk assessment calculator designed specifically for use in total joint arthroplasty. Despite limitations of our study design, and the lower than predicted complication rate, the TKA [total knee replacement] risk calculator represents the novel development of an objective risk assessment tool that does have predictive value, and is a tangible risk assessment device....Physicians may use the calculator to provide more individualized patient counseling and to better stratify risk. (p. 448). 
Researchers at the Mayo Clinic developed a prognostic scoring system that identifies patients at high risk of developing a postoperative infection after total hip or knee replacement surgery (Berbari et al., 2012). The purpose of this risk score is for improved risk-stratified reporting and to target high-risk patients for additional preventative interventions. The Mayo model identified single predicting factors for prosthetic joint infection (PJI). Logistic regression was used to determine the magnitude of each of these associations and then combined into multivariable modeling with logistic regression to determine if there was a pairwise effect between two factors on the risk of infection. Current surgical site infection (SSI) models only take into consideration the patient's American Society of Anesthesiologists (ASA) physical status classification system score; however, the Mayo study has shown that better risk stratification occurs with inclusion of additional important predictors such as BMI, prior arthroplasty, underlying immunosuppression, and prior surgery (Berbari et al., 2012). The authors discuss the importance of accurately accounting for risk, and appropriate risk adjustment will "assure tertiary referral institutions and specialty orthopedic institutions involved in the care of high-risk patients of their ability to perform surgeries on high-risk patients without the fear of being penalized for their relatively higher SSI rates" (p. 779). A limitation of the Mayo model is that in order to calculate a one-month score, data is needed from the preoperative, operative and one-month postoperative time frame. This postoperative information is critical in analyzing infection, as many surgical infections do not set in until the postoperative period. Postoperative data is limited only to those patients that returned to the same facility, or same hospital system if a common electronic 
health record (EHR) system is used. Unless patients are being followed as part of the protocol for a clinical research study, then it is very difficult to achieve accurate postoperative patient information. The Mayo study may not easily be reproduced because patients were followed as part of a research study protocol, something that does not normally occur in the standard delivery of care for patients undergoing surgery. Furthermore, while the factors selected as candidates for the model indeed turned up predictive factors for infection, the question remains whether there are variables that were not included that would have increased the predictive strength of these factors. These non-traditional variables would not have appeared in the initial search of highly predictive individual factors, and may only be predictive in their relation to another factor.

Hospital readmissions are a substantial driver of spending, with all-cause 30-day readmissions costing the US health system more than $\$ 41$ billion annually (Hines $\mathrm{Al}$, Barrett Ml, Jiang Hj \& Steiner Ca, 2011). An application of machine learning techniques has resulted in the highly successful PIECES ${ }^{\mathrm{TM}}$ software (Amarasingham, 2012). PIECES functions on top of a health system's EMR, identifying high-risk patients using a machine learning approach. Patients at high risk for readmission are flagged based on both social and clinical factors found in the patient's chart. This model was implemented in a hospital, where clinical care was tailored for flagged patients, resulting in a $31 \%$ reduction in readmissions.

With bigger data and new techniques for structuring data, disparate data sources will have opportunities to merge into vast repositories. The possibility of more predictive models, and the emergence of "data science" programs within healthcare systems, are 
leading to increased testing of machine learning techniques. While LR will likely continue to be a very well performing method, exploration of new methods with the ability to search through expansive data repositories is an effort that may have significant payoffs when seeking to predict outcomes and custom tailor healthcare delivery in the pursuit of higher value care.

\section{General Overview of LR and its Relation to RA}

Hosmer and Lemeshow (2005) provide an overview of logistic regression (LR) models and discuss the recent explosion in the use of LR over the past decade. They state that from its origination in epidemiological research, LR has gained widespread use and acceptance in biomedical research. LR is typically used to analyze relationships between a dichotomous dependent variable and categorical or continuous independent variables. LR combines the independent variables to estimate the probability that a particular outcome will occur. For example, if we assume that $\mathrm{Z}$ represents the adverse outcome complication, we can create a formula in terms of the probability that a complication occurs, or $\mathrm{Z}=1$, shown below as $\mathrm{p}\left(\mathrm{Z}_{1}\right)$, and the probability that no complication occurs, or $\mathrm{Z}=0$, shown as $\mathrm{p}\left(\mathrm{Z}_{0}\right)$. Therefore, the general mathematical notation is:

$$
\log \left(\frac{p\left(Z_{1}\right)}{p\left(Z_{0}\right)}\right)=\alpha 0+\alpha 1 A+\alpha 2 B+\alpha 3 C
$$

There are areas of overlap between RA and LR. For example, when nominal variable states in RA are re-coded into binary states, RA does resemble LR. Zwick (2011) points out that whether or to what degree the two methods are mathematically equivalent is under investigation. Regardless of the potential mathematical overlap however, RA is computationally distinct. LR maximizes likelihood most often with the Newton-Raphson 
algorithm, while RA uses Iterative Proportional Fitting (IPF) to maximize entropy subject to the constraints of the data. LR software is not designed for exploratory modeling and does not easily handle interactions between multiple variables. Because of this, RA is distinctly useful for searching for novel variables and interaction effects. LR does not generate the lattice of structures nor provide an approach for searching this lattice (Zwick, 2011a). Unlike LR, but like ordinary regression, RA can also analyze continuous dependent variables such as total cost of hip or knee replacement surgery.

\section{An Overview of RA}

Almost without exception, the studies surveyed in the review of the literature generated models using logistic regression methods. While the models were well constructed and tested, there is a trend in the discussions of prior studies urging further testing and validation. One way to validate models is to use a different methodology on similar data and compare the outcomes.

A model developed with RA will not only be an interesting test of comparison of methods, but will test whether additional variables may provide more predictive strength or whether non-hypothesized interaction effects offer additional predictive strength. For example, prior studies using logistic regression had found that APOE was a highly predictive genetic factor of Alzheimer's disease. RA and Alzheimer researchers (Kramer, Westaway, Zwick \& Shervais, 2012) conducted a study using RA which confirmed the gene APOE as highly predictive. However, RA has tentatively surfaced an additional factor-Education - which, when interacting with APOE, generates a model that is more 
predictive than APOE or Education alone. This study looks at the questions of whether there is something similar in total hip and knee replacement surgery.

It can be difficult to visualize what modeling actually does, especially when thinking about the differences between specific modeling methodologies. In the broadest terms, a model is intended to represent reality so as to explain, predict, or control features of that reality which otherwise would be too difficult to observe or manage (Krippendorff 1981). In order to construct a model, data is collected to capture information about a system. Yet this data is inherently very complex, with many degrees of freedom. Using the data directly for prediction is likely to be flawed by overfitting, the postulation of predictive relations that may not be real. A model is a reduction of the data to a simpler structure, and simpler structures generalize better to new data. However, simplifying the data too much will result in a loss of critical information. This presents a tension inherent in the modeling process.

The most common RA approach to modeling is to start with the independence model — which assumes no predictive relations between the IVs and DV—as a reference, and to then search the space of possible models for incremental additions of predictive relations. In RA, this bottom-up approach allows one to construct a model whose complexity is statistically justified, but is still not overly complex.

RA developed from the early works of Ross Ashby (1964), who defined a process for systematically testing whether a seemingly complex constraint could be decomposed into several simpler constraints and then be recomposed to the original constraint without loss. Ashby utilized Shannon's information theory (Shannon, 1948) because it generalized his constraint analysis to probabilistic systems and allowed for the creation of 
an algebra of relations (Krippendorff, 2009). RA was then further developed by Broekstra (1979), Cavallo (1979), Conant (1981), Jones (1985), Klir (1976), Krippendorff (1981), Zwick (2001), and others.

RA assesses hyper-graph structures using either set theoretic (SRA) or information theoretic (IRA) modeling. RA is a data mining method that searches for relations in data, especially nonlinear and higher-order relations. These relations between the variables in the data can have high ordinality, and one need not assume any hypothetical relationship prior to RA. For example, in a study applying RA to genomic data, researchers found that RA can detect gene-gene interactions that other methods could not detect (Shervais, Kramer, Westaway, Cox \& Zwick, 2010).

RA resembles log-linear statistical methods in the social sciences, and has diverse applications including time-series analysis, classification, decomposition, compression, pattern recognition, prediction, control, and decision analysis (Zwick, 2004). RA handles multivariate data with discrete values for nominal variables. Continuous data can be handled by discretizing (binning) into discrete binary or multi-valued states. There are multiple methods for binning data (e.g., rational binning, obvious clustering, and equal sample size). The more states of an IV, the better it can predict the outcome, but the number of states of a variable increases the sample size required, and a trade-off is necessary.

In this study, the information-theoretic (IRA) version is used. IRA turns input data into frequency and probability distributions. IRA decomposes frequency distributions into projections, several of which taken together define a model, which is then assessed for statistical significance. The model maximizes entropy subject to the constraints of the 
model structure. Maximum entropy solutions are often identical to maximum likelihood solutions of other methods.

A "saturated model" represents the data without simplification and assumes the highest ordinality of relations amongst the variables. The "independence model" is the least complex of all models and assumes no predictive relations between the IVs and the DV. For example, a system with three IVs and one DV may be represented as ABCZ (the saturated model), where A, B, and C are the IVs and Z is the DV. No interaction between the IVs and the DVs is called the independence model and would here be characterized by the model ABC:Z. The model ABC:Z is the simplest model possible and signifies that the DV is not predicted by the IVs. Another possible model of the data ABCZ might be $\mathrm{ABC}$ : $\mathrm{AZ}: \mathrm{BZ}: \mathrm{CZ}$ where $\mathrm{A}, \mathrm{B}$, and $\mathrm{C}$ again are the independent variables and $\mathrm{Z}$ is the dependent variable. In the model ABC:AZ:BZ:CZ, A has an effect on Z, B has an effect on $\mathrm{Z}$, and $\mathrm{C}$ has an effect on $\mathrm{Z}$, but there is no interaction effect between the independent variables $\mathrm{A}, \mathrm{B}$, and $\mathrm{C}$. In the independence model $\mathrm{ABC}: \mathrm{Z}$ there are no relations between the IVs and the DV at all, so this model may be said to contain no information. The DV has maximum uncertainty. As used for prediction, an RA model is a conditional probability distribution of the DV, given the IVs. In the present case, for example, this might be expressed as $\mathrm{P}_{\text {model }}\left(\mathrm{Z}_{\mathrm{l}} \mid \mathrm{A}_{\mathrm{i}} \mathrm{B}_{\mathrm{j}} \mathrm{C}_{\mathrm{h}}\right)$.

With four variables, as in the example $\mathrm{ABCZ}$, multiple relations are possible. Each of these possible relations is a structure, without concern for order within or between the relations. There are 19 specific structures for three IVs and one DV. The lattice of structures for four variables is presented in Figure 1 below where the variables are lines and the relations are boxes. Increasing the number of IVs to four results in 167 
specific structures, and increasing the number of IVs to five results in 7,580 specific structures. Data with hundreds of possible IVs will generate a massive lattice of structures. In this study, RA will search through the lattice of structures until it finds IVs that are informative about the probability of a specific outcome.

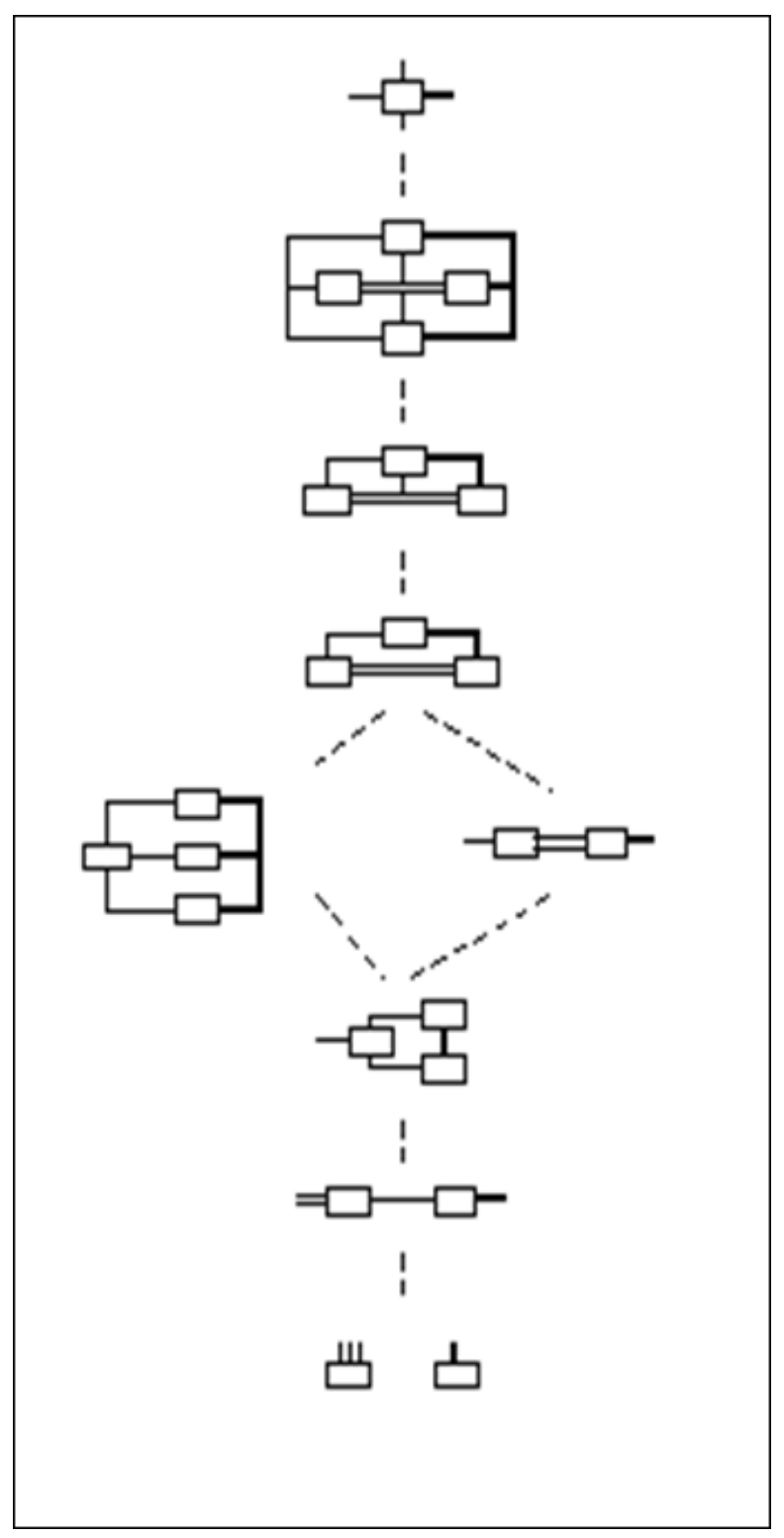

Figure 1. Lattice of structures for 3 IVs and $1 \mathrm{DV}$. 
Continuing with the previous example, assume $\mathrm{ABC}: \mathrm{Z}$ is the independence model for a directed system. The independence model will be the starting model (assuming no relations), and an ascending search will be performed, looking for models through the lattice of structures until difference from independence model and each increase in complexity are not statistically significant. For example, one of the possible model structures in this example search is model ABC:ABZ:CZ. In this model, the A and B variables in the (ABZ) component represent an interaction effect. Every interaction effect will be investigated further to interpret how the input variables combine to predict the output variable.

Looking at multiple models relative to each other offers choices based on predictive strength versus simplicity. Maximally predictive models may be traded in for a simpler, less predictive model. Models that yield high information also are complex and have high degrees of freedom relative to models with less complex structures containing less information and lower degrees of freedom. The "best model" is often somewhere in the middle since overly complex models will do poorly when confronted with new data; this is known as "overfitting."

To avoid overfitting, a good model will capture a maximum amount of the information (the constraint) in the data while still being as simple as possible. After the best models are obtained through an exploratory search, the actual contents of this model — how it predicts the DV given the IV states—must be examined in detail. In Occam, the RA software used in this project, this latter detailed examination is called "fit," to be distinguished from the first step which is called "search." Several RA software applications exist, such as the Construct and Spectral applications (Krippendorff, 1981), 
SAPS (Cellier \& Yandell, 1987, Klir (1976), EDA (Conant, 1988) and Occam (Zwick, 2000). For this project, the third version of the Occam software application is used, Occam3 (Willett \& Zwick, 2004).

Another approach is available which is finer-grained than the variable-based models previously described (Figure 2). Jones (1989) looked at systems in terms of states instead of variables. In variable-based $\mathrm{RA}$, the structures are subsets of $\mathrm{ABC}: \mathrm{Z}$ while in state-based RA, structures specify particular states of one or more variables. In the example of $\mathrm{ABC}: \mathrm{Z}$, where the component $\mathrm{ABC}$ does not predict $\mathrm{Z}$, it may still be that a combination of specific states of $\mathrm{A}, \mathrm{B}$ and $\mathrm{C}$ do in fact predict $\mathrm{Z}$ where the model may be depicted as $A B C: Z: A_{1} B_{1} C_{1} Z$. This more detailed, and in principle more powerful, analysis can be done using state-based RA (Zwick \& Johnson, 2004).

Two levels of refinement in RA will be used in this study: variable-based RA without loops and variable-based RA with loops. The more refined the approach, the more predictive the model may be. It is possible that a more predictive fine-grained model will not have greater complexity (i.e., degrees of freedom) than the coarsergrained variable-based models. These levels will be discussed in greater detail in the methodology chapter. 


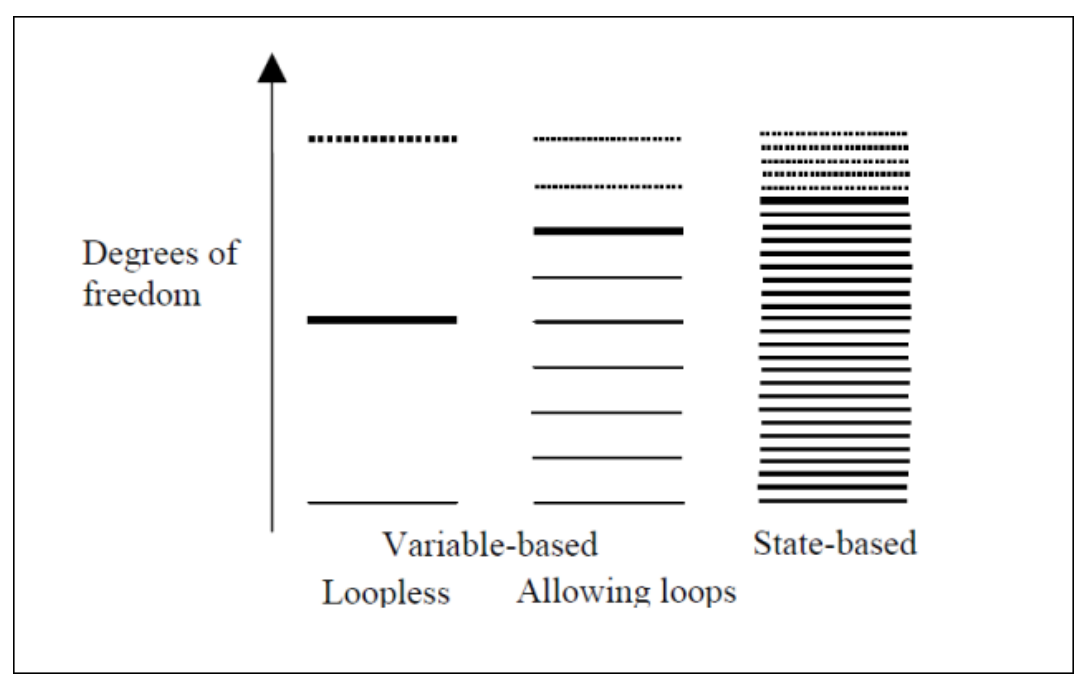

Figure 2. Levels of refinement in RA.

There are different criteria that can be used for model selection. In this project, Akaike Information Criterion (AIC) and Bayesian Information Criterion (BIC) are both used. AIC is a measure of the goodness of fit of a model that trades off the complexity of an estimated model against how much of the information in the data the model captures. BIC is a slightly different way of doing the same thing. BIC is more conservative than AIC and is thus generally preferred over AIC for selecting the best model. Another measure that will be considered when assessing the quality of a model is its information content. In RA, information is a measure of the constraint captured in a model. Since the data always contains $100 \%$ information, and models of the data decompose the relations into smaller components, one must test how far the data can be decomposed while still holding onto enough information to be predictive. While information is scaled from $0-1$, the percent reduction of uncertainty of the dependent variable, represented as $\% \Delta H(D V)$, is the actual reduction of uncertainty achieved by any model and is the actual predictive power of the model. The information measure indicates how much of the information in the data is incorporated into the model, yet the information in the data itself can be small 
or large. This uncertainty reduction measure is calculated by multiplying the information content with the percent reduction of uncertainty of the data. For directed systems, the ability to quantify how predictable the outcome is, given the presence or absence of IVs, is expressed as the reduction of the entropy of $\mathrm{Z}$ (outcome), knowing $\mathrm{A}, \mathrm{B}$ and $\mathrm{C}$ (3 IVs). For IRA, entropy reduction is derived from the conditional probability distribution of the model. Both information and entropy reduction measures do not involve a sample size and are therefore themselves non-statistical (Zwick, 2011a). The reduction in uncertainty is a central measure of RA and something that is not possible with other methods.

A third criterion, the incremental p-value, will supplement AIC and BIC in order to select the model that reduces uncertainty the most, from which every incremental step from the independence model has an acceptable p-value. Knowing the sample size provides for the calculation of the likelihood ratio test (chi-squared, or $\chi^{2}$ ), which then allows the researcher to determine the p-value for entropy reduction, relative to either independence or some other simpler model as reference. This is one of the measures that is used when assessing the trade-off between information and complexity in model selection (Zwick, 2011a). This p-value is the probability, if one has rejected the null hypothesis (that the model is the same as the reference model), that one is incorrect in that rejection. This project will apply RA using the independence model as the reference, where smaller p-values are preferred. As the search continues, new reference models are generated and an incremental p-value will show the statistical significance at each step through the lattice of possible model structures (Zwick, 2004).

In this project, the lattice of all possible models will be searched using a bottom-up approach where the reference model is the independence model. With the 
independence model as the reference, the search up the lattice of structures is an attempt to see if the data justifies a model with more complexity (degrees of freedom) than the independence model. Additionally, many types of models may be assessed in Occam, depending on the specification of search parameters. Going up the lattice, RA generates "parents" of the models at each level in the lattice of structures. A search "width" can be specified, which restricts the number of models retained at each level in the lattice. The maximum number of levels to be searched can also be specified, using the search "levels" parameter.

The literature shows that RA has been validated as a supplementary method, strengthening research when used concurrently with other methods. A problem identified by researchers of the Neural Networks (NNs) methodology is that NNs are often applied on data without knowing what variables are the most valuable as inputs to the NN model, thus resulting in overly complex models. For example, Chambless, Lendaris, and Zwick (2001) applied RA to data and successfully "prestructured" the data, picking out the most important and predictive variables to reduce the neural network's complexity without significant loss of predictive accuracy. Cangur (2009) used RA as a method to augment LR in forecasting mortgage loan statuses. In this study, Cangur was able to improve on what had previously been known to be predictive of mortgage delinquencies, prepayments, defaults, and losses by detecting additional states as well as interaction effects. These interaction effects improved the accuracy of LR models used in the field of loan forecasting. Carletti (2004) generated an RA model that detects complex interactions and predicts health status more effectively than multiple linear regression. Carletti used state-based RA, surfacing literacy level in combination with occupational status and 
intensity of performing strenuous activities as predictive of health status. Mist (2007) also has augmented LR with RA in an application predicting a Chinese Medicine diagnosis from patient pre-treatment questionnaires. In this study, both variable-based and statebased RA were used and interaction effects found. These interaction terms were then introduced into a previously constructed LR model, resulting in an improvement of prediction for three of the most common Chinese Medicine diagnoses.

What this literature demonstrates is that RA can do more than offer a confirmatory role in validating the results of previous studies; it can provide a complimentary approach to other methods, using multiple methods in tandem to strengthen each other. Furthermore, the past studies discussed in this review of the literature have shown that RA provided novel and valuable predictions undetected by the more standard and common statistical modeling techniques, and can be valuable as a stand-alone exploratory method. 


\section{Chapter 3. Methods}

This chapter starts with an overview of the data and provides the definitions of the independent and dependent variables used in this project. Procedural descriptions are provided describing how the data was transformed into the format used in the data analysis. After this overview of the data, the LR and the RA methods as applied in this project are described, as well as explanations of additional calculations used to augment the core methodologies.

\section{The Data}

Data used in this study derives from patients who underwent inpatient surgical procedures of either total hip replacement or total knee replacement at one of seven inpatient hospitals within an integrated healthcare system in a single state. Participant data consists of both hospital billing data and electronic health record system clinical data. Clinical and cost data were matched on the patient's episode identifier, then de-identified and transformed into the variables used in this research project. This project has been granted exempt status from the Portland State University Institutional Review Board.

Effective October 1, 2007, the hospital Uniform Bill (UB) requires hospital claims data to include a present on admit (POA) indicator for each diagnosis field. Diagnosis fields are represented by codes called the International Classification of Diseases, Ninth Revision (ICD-9). ICD-9 codes are created by the World Health Organization, and are the official classification system for surgical, diagnostic, and therapeutic procedures. In this project, ICD-9 codes are used to classify the procedure of 
an elective total hip replacement (ICD-9 code 81.51) or an elective total knee replacement procedure (81.54). ICD-9 codes are also used in this project to classify the Comorbidity IVs and the DV Complication occurring for each hip and knee procedure.

The data was divided into two data sets based on procedure type. The resulting knee data set and hip data set share the same variables, with some differences in comorbidities present. There were 3,205 cases in the hip data set, and 4,336 cases in the knee data set. Because the administrative claims database includes variables that are collected in diverse health systems across the nation, the resulting predictive models developed in this project have the potential for widespread use.

Multiple analyses were conducted in this project, and two combinations of independent variables were assessed: All IVs and Comorbidity IVs. All IVs include the patient Comorbidity IVs as well as Non-Comorbidity IVs.

\section{Comorbidity IVs}

Diagnosis ICD-9 codes and corresponding POA status and rank were merged in order to create the set of Comorbidity IVs. If a diagnosis code appeared as present on admit and rank equaled 1, this was the primary diagnosis and was not included as a comorbidity. For example, it is common to see osteoarthritis as the diagnosis POA with rank $=1$. If a diagnosis code was present on admit with a rank $>1$, then this was considered to be a comorbidity. Each comorbidity present in the data was then turned into a binary variable with the possible states of present (1) or absent (0). If a diagnosis code was present in the data but was not present on admit, then this ICD-9 diagnosis code indicates a complication and was mapped into a complication grouping schema. 
There were 912 individual comorbidity independent variables (Table 103). While the RA method can in principle utilize this many IVs, the data set would need to have a much larger sample size if one wanted to detect interaction effects involving very many IVs.

In the hip data set, 643 of 912 comorbidities occurred in at least 1 of the 3,205 cases, and 270 of these comorbidities were not present in any of the cases. There is a chi-squared rule of thumb that argues for a minimal average cell count of at least 5 . There were 473 comorbidities that occurred in $<5$ cases and 170 comorbidities that occurred in 5-1464 cases (the comorbidity that showed up in 1,464 cases was essential hypertension). The final culled hip data set included 3,205 cases with these 170 Comorbidity IVs.

In the knee data set, 671 of the 912 comorbidities were present in at least 1 case. Using the same rationale as the hip data set, the 483 Comorbidity IVs that were present in $<5$ cases were removed, reducing the total number of Comorbidity IVs to 188 that occurred in 5-2,373 cases (the comorbidity essential hypertension showed up in 2,373 cases). Thus the final knee data set contained 4,336 cases with these 188 Comorbidity $I V s$.

The independent variable called number of risks ( $\mathrm{Nrb}$ ) is tallied by adding up any of the 912 Comorbidity IVs that are indicated as present on admit. While the full 912 IVs are not used in either data set, the Nrb variable in fact is dependent on the presence or absence one or many of the original 912 Comorbidity IVs. In other words, it was "possible" for the total number of risks for a patient to equal 912, even though fewer than 200 comorbidities were retained in the hip and knee data sets. Table 1 shows a sample 
subset of the individual patient Comorbidity IVs. A full list of Comorbidity IVs is available in Table 103.

Table 1. Subset of Comorbidity IVs (Comorbidity IVs from Tier 1 Predictors).

\begin{tabular}{|l|l|}
\hline ID & IV Name \\
\hline Rhd & Other chronic pulmonary heart diseases \\
\hline Ruh & Other and unspecified hyperlipidemia \\
\hline Rrd & Unspecified hypertensive renal disease \\
\hline Rgp & Repair of cystocele with graft or prosthesis \\
\hline Rug & Unspecified glaucoma \\
\hline Rca & Coronary atherosclerosis of native coronary artery \\
\hline Rku & Chronic kidney disease, unspecified \\
\hline Rci & Chronic ischemic heart disease \\
\hline Rhf & Heart failure \\
\hline Rcj & Contracture of joint, lower leg \\
\hline Rco & Chronic obstructive asthma \\
\hline Rmd & Other persistent mental disorders due to conditions classified elsewhere \\
\hline Rpl & Hyperplasia of prostate \\
\hline
\end{tabular}

\section{Non-Comorbidity IVs}

An additional eight independent variables were included that represent, non-binary patient risk factors (age, number of risks, and admit diagnosis) as well as variables suggested as important variables in the literature (Dall et al., 2009; Schroer WC, 2008) describing the delivery system (location, surgeon, surgeon volume, financial class, and day of admission). 
Table 2. Subset of All IVs: Non-Comorbidity Variable Descriptions and IDs.

\begin{tabular}{|c|c|c|c|}
\hline All IVs & & & \\
\hline $\begin{array}{l}\text { Non-Comorbidity } \\
\text { IVs }\end{array}$ & Variable Description (Knee) & Variable Description (Hip) & ID \\
\hline $\begin{array}{l}\text { Location, } \\
\text { multivalued }\end{array}$ & $\begin{array}{l}\text { Location documented as } 1 \text { of } 7 \\
\text { inpatient hospitals within a single } \\
\text { integrated health system }\end{array}$ & Same as Knee & $\mathrm{L}$ \\
\hline $\begin{array}{l}\text { Principal } \\
\text { Surgeon, } \\
\text { multivalued }\end{array}$ & $\begin{array}{l}\text { One of } 64 \text { primary attending } \\
\text { physicians as determined in the } \\
\text { patient's medical chart }\end{array}$ & $\begin{array}{l}\text { Same as Knee, except } 43 \text { primary } \\
\text { attending physicians }\end{array}$ & $\mathrm{S}$ \\
\hline $\begin{array}{l}\text { Surgeon Volume, } \\
\text { Continuous }\end{array}$ & $\begin{array}{l}\text { Total number of procedures per } \\
\text { surgeon over the entire time period } \\
\text { in the data set. Surgeon total volume } \\
\text { ranged from } 1-1,449 \text { cases. }\end{array}$ & $\begin{array}{l}\text { Same as Knee, except volume } \\
\text { ranged from } 1-1,191 \text { cases. }\end{array}$ & $\begin{array}{l}\text { Sv } \\
\text { (binned } \\
\text { to create } \\
\text { Svb, } \\
\text { below) }\end{array}$ \\
\hline $\begin{array}{l}\text { Surgeon Volume, } \\
\text { binned }\end{array}$ & $\begin{array}{l}\text { Surgeon volume counts were binned } \\
\text { into } 12 \text { equal sample sized bins, } \\
\text { then rebinned into } 3 \text { bins: } \\
{[1(1,2,3,4) ; 2(5,6,7,8) ; 3(9,10,11,12)]}\end{array}$ & $\begin{array}{l}\text { Surgeon volume counts were } \\
\text { binned into } 10 \text { equal sample sized } \\
\text { bins, then rebinned into } 3 \text { bins: } \\
{[1(1,2,3,4) ; 2(5,6,7) ; 3(8,9,10)]}\end{array}$ & Svb \\
\hline $\begin{array}{l}\text { Day of Admit, } \\
\text { multivalued }\end{array}$ & $\begin{array}{l}\text { Monday (1), Tuesday (2), } \\
\text { Wednesday (3), Thursday (4), or } \\
\text { Friday (5) }\end{array}$ & $\begin{array}{l}\text { Monday (1), Tuesday (2), } \\
\text { Wednesday (3), Thursday (4), or } \\
\text { Friday (5), Saturday (6) }\end{array}$ & $\mathrm{Da}$ \\
\hline $\begin{array}{l}\text { Financial Class, } \\
\text { multivalued }\end{array}$ & $\begin{array}{l}\text { Medicare (1), Commercial (2), } \\
\text { Medicaid (3), Workers Comp (4), } \\
\text { Other Government (5), Self or Other } \\
\text { (6) }\end{array}$ & Same as Knee & $\mathrm{Fc}$ \\
\hline Age, continuous & $\begin{array}{l}\text { Patient age at time of admission to } \\
\text { hospital. Age ranges from 32-94 } \\
\text { years old. }\end{array}$ & $\begin{array}{l}\text { Same as Knee, except Age ranges } \\
\text { from } 15-96 \text { years old. }\end{array}$ & $\begin{array}{l}\text { Age } \\
\text { (binned } \\
\text { to create } \\
\text { Ageb, } \\
\text { below) } \\
\end{array}$ \\
\hline Age, binned & $\begin{array}{l}\text { Data was first binned into } 12 \text { equal } \\
\text { sample size bins, then re-binned into } \\
3 \text { equal size bins as follows: } \\
{[1(1,2,3,4) ; 2(5,6,7,8) ; 3(9,10,11,12)]}\end{array}$ & Same as Knee & Ageb \\
\hline $\begin{array}{l}\text { Admit Diagnosis, } \\
\text { multivalued }\end{array}$ & $\begin{array}{l}\text { This is the primary (coded) } \\
\text { diagnosis present on admit. }\end{array}$ & Same as Knee & $\mathrm{Ad}$ \\
\hline $\begin{array}{l}\text { Number of Risks, } \\
\text { continuous }\end{array}$ & $\begin{array}{l}\text { Individual diagnosis codes coded as } \\
\text { present on admit }=\text { Y. Total number } \\
\text { of risks per case ranged from } 0 \text { to } \\
18 \text {. }\end{array}$ & $\begin{array}{l}\text { Same as Knee, except risks per case } \\
\text { ranged from } 0 \text { to } 19 .\end{array}$ & $\begin{array}{l}\mathrm{Nr} \\
\text { (binned } \\
\text { to create } \\
\mathrm{Nrb}, \\
\text { below) }\end{array}$ \\
\hline $\begin{array}{l}\text { Number of Risks, } \\
\text { binned }\end{array}$ & $\begin{array}{l}\text { Number of risks were binned into } 8 \\
\text { equal sample sized bins, then } \\
\text { rebinned into } 3 \text { equal sample size } \\
\text { bins as follows: } \\
{[1(1,2) ; 2(3,4) ; 3(5,6,7,8)]}\end{array}$ & Same as Knee & $\mathrm{Nrb}$ \\
\hline
\end{tabular}

This project uses de-identified data and does not use or disclose specific names of

these variables. Location or surgeon names, in combination with other variables, could be 
used to identify a patient and can be considered protected health information (HIPAA, 2009).

\section{Binning Continuous IVs}

Not only are there different sets of individual patient comorbidities present in the hip and knee populations, but the average age for knee patients is higher at 67.1 years versus 64.72 years for hip. The knee patients had an average of 3 patient comorbidities, while the hip patients averaged 2.81. Due to the differences in hip and knee populations, binning of the IVs was performed independently per data set. The independent variables age (Age), surgeon volume (Sv), and number of risks $(\mathrm{Nr})$ were continuous variables that were discretized into the binned variables Ageb, Svb, and Nrb. These IVs were divided into three equal sample-sized bins, which will allow for the detection of nonlinear effects, within the hip data set, and then again in the knee data set as shown in Table 3 below.

Table 3. Binned IVs for the Continuous Variables: Number of Risks, Age, and Surgeon Volume.

\begin{tabular}{|c|c|c|c|c|c|}
\hline \multicolumn{6}{|c|}{ Number of Risks Binned (Nrb) } \\
\hline \multicolumn{3}{|c|}{ Knee } & \multicolumn{3}{|c|}{ Hip } \\
\hline Bin & Range & Frequency & Bin & Range & Frequency \\
\hline 1 & $0-1$ & 1309 & 1 & $0-1$ & 1,111 \\
\hline 2 & $2-3$ & 1474 & 2 & $2-3$ & 1,081 \\
\hline 3 & 4-18 & 1553 & 3 & 4-19 & 1,013 \\
\hline \multicolumn{6}{|c|}{ Age Binned (Ageb) } \\
\hline \multicolumn{3}{|c|}{ Knee } & \multicolumn{3}{|c|}{ Hip } \\
\hline Bin & Range & Frequency & Bin & Range & Frequency \\
\hline 1 & $32-62$ & 1,490 & 1 & $15-59$ & 1,090 \\
\hline 2 & $63-71$ & 1,411 & 2 & $60-69$ & 1,027 \\
\hline 3 & $72-95$ & 1,435 & 3 & $70-96$ & 1,088 \\
\hline \multicolumn{6}{|c|}{ Surgeon Volume Binned (Svb) } \\
\hline \multicolumn{3}{|c|}{ Knee } & \multicolumn{3}{|c|}{ Hip } \\
\hline Bin & Range & Frequency & Bin & Range & Frequency \\
\hline 1 & $1-479$ & 1,444 & 1 & $1-461$ & 1,067 \\
\hline 2 & $550-922$ & 1,518 & 2 & $479-778$ & 761 \\
\hline 3 & $987-1449$ & 1,374 & 3 & $779-1191$ & 1,377 \\
\hline
\end{tabular}


The primary purpose of the age variable in this project is to determine whether age has an effect at all. The standard practice to test if an IV is predictive is to use uniformly sampled bins. The more uniform an IV distribution is, the more predictive or explanatory it can be. So the question asked in this dissertation is whether age makes a difference. A subsequent question, not pursued in this project, could be to look at whether using bin definitions more common in the field (e.g., binning age by non-enrollment or enrollment in Medicare) might provide more useful or enhanced predictions.

\section{Dependent Variables (DVs)}

Four DVs were constructed for this project and are summarized below in Table 4. These DVs include adverse events Complication and Readmissions, and high cost indicators of Skilled Nursing Facility discharges and Total Cost.

Table 4. Description of the Original Dependent Variables and Subsequent Transformed (binned) Final Dependent Variables.

\begin{tabular}{|c|c|c|c|}
\hline $\begin{array}{l}\text { Dependent } \\
\text { Variables (DVs): }\end{array}$ & Variable Description (Knee) & Variable Description (Hip) & ID \\
\hline Complication & $\begin{array}{l}\text { Any (coded) diagnosis that was not } \\
\text { present on admit. }\end{array}$ & Same as Knee. & $\mathrm{Cp}$ \\
\hline $\begin{array}{l}\text { Discharge } \\
\text { Disposition }\end{array}$ & $\begin{array}{l}\text { Home Self (1), SNF (2), Home } \\
\text { Health (3), Swing Bed (4), IP } \\
\text { Rehab (5), ICF (6), Short Hospital } \\
\text { (7), Expired (8), AMA (9) }\end{array}$ & $\begin{array}{l}\text { Same as Knee, except no AMA } \\
\text { state }\end{array}$ & $\begin{array}{l}\text { (used to } \\
\text { create } \\
\text { DV SNF) }\end{array}$ \\
\hline $\begin{array}{l}\text { Skilled Nursing } \\
\text { Facility (SNF) } \\
\text { Discharge }\end{array}$ & $\begin{array}{l}\text { No SNF }=1, \text { SNF }=2 \\
{[1(1,3,4,5,6,7,8,9) ; 2(2)]}\end{array}$ & $\begin{array}{l}\text { No } \mathrm{SNF}=1, \mathrm{SNF}=2 \\
{[1(1,3,4,5,6,7,8) ; 2(2)]}\end{array}$ & SNF \\
\hline $\begin{array}{l}\text { Readmission, } \\
\text { multivalued } \\
\text { (within } 90 \text { days) }\end{array}$ & $\begin{array}{l}\text { Readmission (Yes/No) according to } \\
\text { Premier inpatient quality reporting } \\
\text { system. There were } 113 \text { total } \\
\text { readmissions within } 90 \text { days to the } \\
\text { same hospital as original surgery. }\end{array}$ & $\begin{array}{l}\text { Same as Knee, except } 87 \text { total } \\
\text { readmissions within } 90 \text { days to the } \\
\text { same hospital as original surgery. }\end{array}$ & $\operatorname{Re}$ \\
\hline $\begin{array}{l}\text { Total Cost } \\
\text { (continuous) }\end{array}$ & $\begin{array}{l}\text { Hospital costed "total cost per } \\
\text { case." Range } \$ 5,945 \text { - } \$ 96,880 \text { per } \\
\text { case. }\end{array}$ & $\begin{array}{l}\text { Same as Knee, except Range } \\
\$ 11,147-\$ 71,264 \text { per case. }\end{array}$ & $\begin{array}{l}\text { (used to } \\
\text { create } \\
\text { DV Tcb) }\end{array}$ \\
\hline $\begin{array}{l}\text { Total Cost } \\
\text { (binned) }\end{array}$ & $\begin{array}{l}\text { Total Cost was binned first into } 12 \\
\text { equal sample sized bins, then } \\
\text { rebinned into } 3 \text { bins as follows: } \\
{[1(1,2,3,4) ; 2(5,6,7,8) ; 3(9,10,11,12)]}\end{array}$ & Same as Knee & Tcb \\
\hline
\end{tabular}


The DV Complication, represented by the ID "Cp" (Table 4) was created by looking at the ICD-9 diagnosis codes with a POA indicator of 0 , indicating the diagnosis was acquired after admission to the hospital. The knee data set contained 913 individual complications across 205 cases. The complication rate for the knee data set is then $205 / 4336$ or $4.7 \%$. In the hip data set, there were 790 complications present across 164 cases with a rate of $5.2 \%$.

The Skilled Nursing Facility (SNF) DV is constructed from the discharge disposition filed in the administrative database. There are nine possible discharge locations in the data: home health, skilled nursing facility (SNF), home self-care, inpatient rehab, swing bed, short term hospital, intermediate care facility (ICF), expired, and AMA (knee only). If the discharge location was SNF, then the DV SNF was assigned a 2. If the discharge location was any of the other locations, the value of the DV was assigned a 1 . In the knee data, $17.6 \%$ of patients were discharged to a SNF and $14.3 \%$ of hip patients were discharged to a SNF.

The Readmission DV is reported out of a nationally standardized quality reporting database called Premier. Premier utilizes a methodology for counting related readmissions. Premier is a common quality reporting system for hospitals across the nation and therefore provides reproducible analysis for readmissions (Grosso, 2012). While easily reproducible, this methodology of counting readmissions is limited in that it is counting only readmissions to an inpatient stay at the same hospital as discharge and therefore does not include visits to the ED or admissions to a different inpatient hospital than originally discharged from. For this project's Readmission DV, if a patient had one or more readmissions within 90 days to the same hospital as discharge, then this case was 
assigned a 1. If no readmission was detected, then the case was assigned a 0 . This method results in many fewer readmissions counted and a very sparse DV with $2.6 \%$ of knee patients readmitted within 90 days from discharge and $2.7 \%$ of hip patients. Premier is adopting a new readmission methodology which will look across inpatient hospitals. In this new method, it will be possible to count readmissions that occurred at locations different from the discharging location.

Binning the Total Cost DV and Calculating Expected Values

Total Cost is a continuous DV with dollar amounts that ranged from $\$ 8,553$ to $\$ 96,880$. There was an average Total Cost of $\$ 18,502$ in the knee data, and a range of $\$ 11,147$ to $\$ 71,264$, with an average of $\$ 18,593$, in the hip data. Total Cost was binned into three equal sample-size bins (low, medium, high).

Table 5. Binned IV (Tcb) for Total Cost Variable.

\begin{tabular}{|r|r|r|r|r|}
\hline \multicolumn{5}{|l|}{ Knee, Total Cost Binned (Tcb) } \\
\hline Bin & Min Cost & Max Cost & Average Cost & Frequency \\
\hline 1 & $\$ 8,553$ & $\$ 16,780$ & $\$ 15,269$ & 1446 \\
\hline 2 & $\$ 16,781$ & $\$ 19,139$ & $\$ 17,922$ & 1445 \\
\hline 3 & $\$ 19,140$ & $\$ 96,880$ & $\$ 22,318$ & 1445 \\
\hline \multicolumn{2}{|l|}{ Hip, Total Cost Binned (Tcb) } \\
\hline Bin & Min Cost & Max Cost & Average Cost & Frequency \\
\hline 1 & $\$ 11,147$ & $\$ 16,768$ & $\$ 15,244$ & 1068 \\
\hline 2 & $\$ 16,772$ & $\$ 19,192$ & $\$ 17,997$ & 1069 \\
\hline 3 & $\$ 19,195$ & $\$ 71,264$ & $\$ 22,534$ & 1068 \\
\hline
\end{tabular}

Each of these bins has an average cost, and along with the product of the probabilities of each bin, an expected value is calculated and used in the interpretation of the results for the Total Cost DV. Binning the DV in this way is referred to as "b-systems" analysis, where continuous values are derived from a binned DV (Zwick, Fusion, \& Wilmot). The model's conditional probability distribution includes the 
calculated probability of each of the model's IV states for the low-cost (bin 1), mid-cost (bin 2), and high cost (bin 3) bins. The product of the probabilities of each bin and each bin's average Total Cost was used to calculate an Expected Value (predicted Total Cost) for each IV state:

$$
\text { Expected Value }=\frac{p(\mathrm{Tcb} 1) \times \operatorname{Avg}(\mathrm{Bin} 1)+p(\mathrm{Tcb} 2) \times \operatorname{Avg}(\mathrm{Bin} 2)+p(\mathrm{Tcb} 3) \times \operatorname{Avg}(\mathrm{Bin} 3)}{100}
$$

\section{Associations between the DVs}

A preliminary analyses was performed looking at the amount of uncertainty reduced for each of the DVs, given the other DVs (set as IVs). There is some association between the DVs. For example, in the knee data set, Total Cost (Tcb) reduces the uncertainty $(\Delta \mathrm{H})$ of Complication $(\mathrm{Cp})$ by $8.67 \%$ (Table 6$)$.

Table 6. Association of DVs by $\% \Delta H$ in Knee Data Set

\begin{tabular}{|c|c|c|c|c|}
\hline \multirow{4}{*}{ Complication (Cp) } & MODEL & $\Delta \mathrm{df}$ & $\Delta \mathrm{BIC}$ & $\% \Delta \mathrm{H}$ \\
\hline & Tcb Cp & 2 & 125.95 & 8.67 \\
\hline & SNF Cp & 1 & 47.93 & 3.42 \\
\hline & $\operatorname{Re} C p$ & 1 & 0.38 & 0.53 \\
\hline \multirow{3}{*}{ Skilled Nursing (SNF) } & Tcb SNF & 2 & 119.75 & 3.39 \\
\hline & $\mathrm{Cp}$ SNF & 1 & 47.93 & 1.40 \\
\hline & Re SNF & 1 & 12.78 & 0.53 \\
\hline \multirow{3}{*}{ Readmission (Re) } & $\mathrm{Tcb} \operatorname{Re}$ & 2 & 6.02 & 2.17 \\
\hline & SNF Re & 1 & 12.78 & 2.02 \\
\hline & $\mathrm{Cp} \operatorname{Re}$ & 1 & 0.38 & 0.84 \\
\hline \multirow{3}{*}{ Total Cost (Tcb) } & Cp Tcb & 2 & 125.95 & 1.50 \\
\hline & SNF Tcb & 2 & 119.75 & 1.43 \\
\hline & $\operatorname{Re} \mathrm{Tcb}$ & 2 & 6.02 & 0.24 \\
\hline
\end{tabular}

In the hip data set, Total Cost (Tcb) also has an association with Complication (Cp) with a $\% \Delta H$ of 7.93 , and with Skilled Nursing Facility (SNF) with a $\% \Delta H$ of 7.37 (Table 7). 
Table 7. Association of DVs by $\% \Delta H$ in Hip Data Set

\begin{tabular}{|c|c|c|c|c|}
\hline \multirow{4}{*}{ Complication (Cp) } & MODEL & $\Delta \mathrm{df}$ & $\Delta \mathrm{BIC}$ & $\% \Delta \mathrm{H}$ \\
\hline & Tcb Cp & 2 & 85.57 & 7.93 \\
\hline & SNF Cp & 1 & 27.50 & 2.77 \\
\hline & $\operatorname{Re} \mathrm{Cp}$ & 1 & -6.65 & 0.11 \\
\hline \multirow{3}{*}{ Skilled Nursing (SNF) } & Tcb SNF & 2 & 176.61 & 7.37 \\
\hline & Cp SNF & 1 & 27.50 & 1.36 \\
\hline & Re SNF & 1 & -6.17 & 0.07 \\
\hline \multirow{3}{*}{ Readmission ( $R e)$} & Tcb Re & 2 & -0.91 & 1.91 \\
\hline & SNF Re & 1 & -6.17 & 0.24 \\
\hline & $\mathrm{Cp} \mathrm{Re}$ & 1 & -6.65 & 0.18 \\
\hline \multirow{3}{*}{ Total Cost (Tcb) } & SNF Tcb & 2 & 176.61 & 2.74 \\
\hline & Cp Tcb & 2 & 85.57 & 1.45 \\
\hline & $\operatorname{Re} \mathrm{Tcb}$ & 2 & -0.91 & 0.22 \\
\hline
\end{tabular}

\section{Formatting Files for Input into Occam Software}

The data files were transformed into a format accepted by the Occam software. These Occam input files specify the variables and include the data to be analyzed. The data file is a plain-text ASCII file saved in a ".txt" format. Initial input files were created for both hip and knee separately. The All IVs and the DVs looked the same; however, the specifications of the Comorbidities are different, as the data sets have different sets of Comorbidity IVs and of course the data itself is different in each. Below is an example of one of the input files. After the variable name, the first number indicates the cardinality (number of states) of the variable (e.g., there are 7 Location states); the second number is 1 for an IV, 2 for a DV, and 0 for "ignore this variable"; the third string of characters is a short name for the variable; for some variables, a rebinning string specifies aggregation of multiple states into fewer rebinned states (e.g., for Age binned, previous bins 1,2,3,4 are collapsed into new bin 1). 
Table 8. Example of an Input File for Occam.

\begin{tabular}{|c|c|c|c|c|c|}
\hline \multirow{2}{*}{\multicolumn{6}{|c|}{ :nominal }} \\
\hline Location & & & & & \\
\hline Principal Surgeon & \multicolumn{5}{|l|}{$, 43,1, \mathrm{~s}$} \\
\hline Day of admit & \multicolumn{5}{|l|}{$, 6,1, \mathrm{da}$} \\
\hline Financial Class & $6,1, \mathrm{fc}$ & & & & \\
\hline Age binned & 12,1,ageb, & \multicolumn{4}{|c|}{$[1(1,2,3,4) ; 2(5,6,7,8) ; 3(9,10,11,12)]$} \\
\hline Surgeon Volume Binned & $, 10,1, \mathrm{svb}$ & \multicolumn{4}{|c|}{$[1(1,2,3,4) ; 2(5,6,7) ; 3(8,9,10)]$} \\
\hline Admit Diagnosis & $, 39,1, \mathrm{ad}$ & \multirow{2}{*}{\multicolumn{4}{|c|}{$[1(1,2) ; 2(3,4) ; 3(5,6,7)]$}} \\
\hline Number of Risks Binned & $, 7,1, \mathrm{nrb}$ & & & & \\
\hline Complication & $, 2,2, \mathrm{cp}$ & & & & \\
\hline Skilled Nursing Facility & $, 8,0, \mathrm{dd}$ & \multicolumn{4}{|c|}{$[1(1,3,4,5,6,7,8) ; 2(2)]$} \\
\hline Total Cost Binned & $, 12,0, \mathrm{tcb}$ & & & & \\
\hline \multicolumn{6}{|l|}{ Readmission 90 Days } \\
\hline RISK_185 & \multicolumn{5}{|l|}{$, 2,1, \mathrm{Rnp}$} \\
\hline RISK_238.75 & \multicolumn{5}{|l|}{$, 2,1, \mathrm{Rmp}$} \\
\hline RISK_244 & \multicolumn{5}{|l|}{$, 2,1, \mathrm{Rh}$} \\
\hline RISK_255.41 & \multicolumn{5}{|l|}{$, 2,1, \operatorname{Rgd}$} \\
\hline RISK_266.2 & \multicolumn{5}{|l|}{$, 2,1, \mathrm{Rbc}$} \\
\hline RISK_268.2 & \multicolumn{5}{|l|}{$, 2,1, \mathrm{Rou}$} \\
\hline RISK_268.9 & \multicolumn{5}{|l|}{$, 2,1, \operatorname{Rvd}$} \\
\hline RISK_272 & \multicolumn{5}{|l|}{$, 2,1, \mathrm{Rli}$} \\
\hline \multicolumn{6}{|l|}{ :no-frequency } \\
\hline \multicolumn{6}{|l|}{ :data } \\
\hline \#nrb & $\mathrm{cp}$ & Rnp & $\mathrm{Rmp}$ & $\mathrm{Rh}$ & Rhy \\
\hline 6 & 1 & 0 & 0 & 0 & 0 \\
\hline 1 & 0 & 0 & 0 & 0 & 0 \\
\hline 2 & 0 & 0 & 0 & 0 & 0 \\
\hline 3 & 0 & 0 & 0 & 0 & 0 \\
\hline 5 & 0 & 0 & 0 & 0 & 0 \\
\hline 7 & 0 & 0 & 0 & 0 & 0 \\
\hline 1 & 0 & 0 & 0 & 0 & 0 \\
\hline 4 & 0 & 0 & 0 & 0 & 0 \\
\hline
\end{tabular}

\section{Variable Reduction per DV}

As discussed above, the criterion of occurrence in at least five cases was used to reduce the IVs to188 in the knee data and 170 in the hip data. Preliminary analyses indicated that a further reduction of IVs was necessary, since even simple models included many IV states with zero or very low frequencies. To do this additional variable reduction, a level $=1$ loopless search was performed to assess the predictive strengths, expressed in $\% \Delta \mathrm{H}$ reduction, of the 188 and 170 IVs. A single predicting IV was 
considered to have predictive value if its $p$-value was $\leq .05$. An overview of IV selection, and the resulting number of IVs per analysis, is provided in each of the results sections. Additionally, sorting these predictive IVs by greatest $\% \Delta \mathrm{H}$ to least showed the single predicting IVs with the greatest value. This variable reduction method was performed for hip and knee data sets for each of the DVs. IVs that were found to have predictive value in the literature were retained for the searches as well, and are summarized in Table 9 below.

Table 9. Literature-based IVs Retained in all RA Exploratory Searches.

\begin{tabular}{|l|l|l|c|c|}
\hline ICD-9 Code & ICD-9 Description & ID & Knee & Hip \\
\hline RISK 250 & Diabetes mellitus & Rdi & yes & yes \\
\hline RISK 250.4 & Diabetes with renal manifestations & Rdr & yes & no \\
\hline RISK 250.5 & Diabetes with ophthalmic manifestations & Rdo & yes & no \\
\hline RISK 250.6 & Diabetes with neurological manifestations & Rdn & yes & yes \\
\hline RISK 278 & Overweight, obesity and other hyperalimentation & Roo & yes & yes \\
\hline RISK 278.01 & Morbid obesity & Rmo & yes & yes \\
\hline RISK 278.02 & Overweight & Rov & yes & yes \\
\hline RISK 401.1 & Benign essential hypertension & Rbe & yes & yes \\
\hline RISK 401.9 & Unspecified essential hypertension & Rhe & yes & yes \\
\hline RISK 414 & Other forms of chronic ischemic heart disease & Rci & yes & yes \\
\hline RISK 414.01 & Coronary atherosclerosis of native coronary artery & Rca & yes & yes \\
\hline RISK 428 & Heart failure & Rhf & yes & yes \\
\hline RISK 428.3 & Diastolic heart failure & Rdh & yes & yes \\
\hline RISK 428.32 & Chronic diastolic heart failure & Rdf & yes & no \\
\hline RISK 443 & Other peripheral vascular disease & Rpe & yes & yes \\
\hline RISK 443.9 & Peripheral vascular disease, unspecified & Rpv & yes & yes \\
\hline RISK 491.2 & Obstructive chronic bronchitis & Rcb & yes & no \\
\hline RISK 491.9 & Unspecified chronic bronchitis & Rbh & yes & no \\
\hline RISK 492.8 & Other emphysema & Rem & yes & yes \\
\hline RISK 493 & Asthma & Ras & yes & no \\
\hline RISK 493.2 & Chronic obstructive asthma & Rco & yes & yes \\
\hline RISK 493.9 & Asthma unspecified & Rua & yes & yes \\
\hline RISK 496 & Chronic airway obstruction, not elsewhere classified & Rao & yes & yes \\
\hline
\end{tabular}

\section{Rationale for No Training/Test Split}

Preliminary analyses were conducted with training/test splits, but these resulted in changes to the \%correct measure that were small and misleading. While performing 
training/test splits is common in machine learning research, it is often done with larger sample sizes and fewer variables. This project's primary objective was exploratory modeling, in which it does not seek to report definitive results, but offers variables and models which should be subjected to subsequent confirmatory testing. Training/test splits were thus not considered to be a necessary component of this project, and were not pursued further.

\section{Logistic Regression Analysis}

Prior to using RA for exploratory modeling and selecting the best predictive models, a preliminary comparison of RA with LR was conducted. Using LR, the researcher can specify the predictor variables expected to be useful in predicting the outcome. This is considered a confirmatory, and not an exploratory, approach. In this project, LR (as programed in R) was used in a confirmatory approach in order to validate both the data used in this project and the RA method. In this analysis, binomial LR analysis was performed to recreate a previous study where the outcomes assessed were binary DVs. The goal of this analysis was confirmatory - to test an a priori hypothesis of the predictive strength of a model that includes predictor IVs predetermined from the literature to be useful in predicting postoperative complications and non-homebound discharge.

Next, the relatively modest exploratory capabilities of LR were used to compare the relative abilities of LR and RA in finding the most predictive models. This analysis was conducted using a stepwise approach. In the "backward stepwise" (versus forward) method of LR exploratory analysis, the saturated model (all variables) is used with no 
specifications of interactions. This stepwise LR approach is more comparable than purely confirmatory LR modeling to the exploratory modeling using RA.

\section{Regular Logistic Regression}

In order to evaluate the logistic regression models, the "stats" package from $\mathrm{R}$ was used (R. Core Team., 2016). The variables included in the LR analysis were converted into "factors" in order for $\mathrm{R}$ to treat them as nominal variables.

LR falls within a broader category of models called "general linear models" that includes ordinary regression, log-linear regression, and ANOVA. In this analysis, the "glm" (used to fit "general linear models") function in R was used. The "glm" function is specified through the use of arguments. Arguments specified under family functions allowed the creation of models in the binomial family and of the "logit" type.

A reference point, in this case the "null model," was created as an object from the data. In this reference model the DV was specified, but no predicting variables were specified. The "null model" as reference allowed for the calculations of measures necessary for comparison across LR and RA methods, such as $\Delta$ df. For comparison, the reference models are shown for both Occam and R:

- R: $\quad$ Tcb $~ 1$

- Occam: IV : Tcb

A second model was then created as a model object from the data. This second model was the model to evaluate against the reference model. Here, the predictor IVs were specified, including any hypothesized interaction terms. Again, here are models written in both forms: 
- R:

$\mathrm{Tcb} \sim \mathrm{Rdi}+\mathrm{Rdn}+\mathrm{Roo}+\mathrm{Rmo}$

- Occam: IV : RdiTcb : RdnTcb : RooTcb : RmoTcb

With the null model as the reference, and the model to evaluate defined, the following measures were calculated:

- $\Delta$ df: take the difference in DF values of the two models.

- $\Delta \mathrm{AIC}$ : take the difference in AIC values of the two models.

- $\Delta \mathrm{LR}$ : take the difference in deviance values of the two models.

- alpha: calculate an "ANOVA" table for the two models with test type "Chisq" and select the desired value from the resulting table.

\section{Stepwise Logistic Regression}

The previous method measures a specified model to the null reference model, but does not explore or propose additional models. In order to find a new and perhaps more predictive model, a stepwise search, using the "step" function from the R "stats" package, was used. This function can search either upward or downward, adding or removing components from a model within specified boundaries (described below). In this stepwise approach, the search considered all single steps that could be made from the model and then selected the best of those by the specified metric. This process was repeated until no better models were found, or a boundary or other termination condition was met. The stepwise search in $\mathrm{R}$ is similar to the Occam searches with the parameter "width" $=1$, but only considering LR models and not Occam's loopless or all-models searches.

To begin a stepwise search for an LR model, models were prepared as described previously, using the "glm" and the binomial family arguments. Using this stepwise method, search direction can be upward from the null model (independence), or downward from the model containing all the variables (saturated model), or searching in both directions from some model "in the middle" that contains some of the variables in 
the data. In the LR analysis for this project, both upward and downward searchers were performed.

The "step" function uses AIC by default as its criterion for model comparison; however, the function can also be modified to instead select based on BIC. Additionally, an upper boundary for the search scope was specified by providing a model; for instance, a model that includes all variables of interest but excludes others in the data. The lower boundary was also be specified, either as the null model (the default) or another model. The stepwise search then results in the model that has been found through this series of single-variable additions and/or subtractions (steps), with the best value for AIC.

\section{Determining the Best RA Models}

In order to search for the most predictive models for each of the DVs, the reference model used was always the bottom, or the "independence model," with a search direction of "up." This method allows for moving up the lattice of structures, away from independence (no relations) toward a more complex model. Unjustified complexity results in large cumulative or incremental alphas that indicate the search has gone too far. In this project, cumulative p-values $\geq 0.05$ indicated the model was more complex than warranted, and a model lower in the lattice was subsequently selected.

Models without loops, and models allowing for loops, were both considered and are provided in the model search results. During the search process, models were sorted by $\triangle \mathrm{BIC}$, with a preference for larger values. Each search was initially specified to keep three models at each level, and to search up to seven levels in the lattice for both loopless searches (coarse models), and searches allowing for models with loops (fine-grained 
searches). For the loopless search, a seven-level search means that models with interaction effects involving up to seven IVs could in principle be considered. For the search that allows loops, models that add seven steps of complexification of the independence model are considered, where a step is either adding another variable to an interaction effect that is already being modeled or adding an additional predicting component to the model.

If the search results indicated the best model by either BIC or AIC criterion was at the topmost level (Level 7 according to the initial settings) then additional searches were conducted looking higher than seven levels. The number of levels varied per search, but the protocol used always added levels until it was certain that the best models by BIC or AIC were not at the highest level that was examined. Additionally, a similar protocol was used for determining the width of the searches. Once a level was determined, width was increased to see if a better model was found. Once a model remained unchanged, either by increasing search levels or search widths, then this was considered the "best model" for that search.

The best models that are the primary results of this project are selected according to BIC. The benefit of using the conservative BIC model selection criterion-as opposed to, say, using the AIC criterion - is that overfitting is unlikely; in being conservative, however, it is possible that the models selected were not aggressive enough, with a consequence of missing real interactions. (AIC was, however, used in RA-LR comparisons, because the LR software used this criterion.) BIC models are always "cumulatively" statistically significant; i.e., their difference from independence is always significant. In addition, in all of the BIC models reported there is always a path where 
every step of increasing complexity from independence to the model is statistically significant, the significance of each step being given by the Occam measure Incremental Alpha.

\section{Fitting the RA Models to the Data \& Identifying Important Model IV States}

The "fit" action of Occam displays the model's internal structure, the conditional probability distribution for the DV, given the predicting IVs. In this project, best models were obtained for 16 searches, each resulting in a conditional probability distribution. Each distribution shows the conditional DV \% for each of the IV composite states for the model and include frequencies and observed probabilities calculated from the data, as well as the calculated probabilities from the model. The frequency of all the IV states observed in the data, the data's marginal probabilities, and the calculated IV conditional probabilities of the model are used for selecting important IV states, discussed further in the next section.

The model fit analysis also displays the individual model component's projections and is frequently informative. Each individual component may be a single variable, or more than one variable if a relation (interaction term) was found in the model.

For both the model's full table and the individual components' tables, if the conditional probabilities for particular IV states are higher or lower than the margins, then the IVs have provided new (predictive) information. In this project, conditional probabilities that appear different from the margins are indicted by the blue and orange shaded cells. Whether or not this effect size is significant is assessed by a chi-square p-value, calculated from the margins, the IV state's conditional probabilities, and the 
frequencies for that IV state. This p-value indicates the statistical significance of the difference between the conditional distribution for particular IV states, namely q(DV|IV), and the sample margins, namely $\mathrm{p}(\mathrm{DV})$. The important IV states for each of the models is then communicated in a decision tree when possible. If the number of variables and their number of states make the decision tree too large for inclusion, then the decision tree is omitted. The decision trees provide a more intuitive visualization of the model's predictors and their effect on the DV.

\section{Selection Criteria for Important Model IV States}

All observed IV states for each of the models are included in the original RA output from Occam. In this project, a criterion is used that considers only IV states that occur in 10 or more cases (freq $\geq 10$ ). This decision is suggested by the Chi-square rule of thumb that calls for on average at least five values per cell. For DVs with cardinalities of two, requiring at least 10 cases doubles this guideline and imposes it on every IV state; this implements a conservative position on making assertions from the model conditional probability distribution. The position thus adopted here thus is that a p-value is assessed only if an IV state occurs in 10 or more cases. If $\mathrm{p} \leq 0.05$, then the IV state is retained; otherwise the IV state is omitted.

Of these remaining IV states, a "risk ratio" is then calculated. If the IV state has a ratio $\geq 1.10$, it is considered to be a higher-risk state, and if the ratio is $\leq 0.90$, then it is considered to be a lower-risk state. Any IV state that is between 0.91 and 1.09 is considered to have a small effect size and is thus not considered an important IV state. These risk ratios are a primary measure of effect size, and while $5 \%$ is often considered 
standard for an important effect size, this project doubles it to $10 \%$, which is another conservative choice.

\section{Calculating Risk Ratios}

The risk ratio of an IV state is a measure of its effect size, whereas the p-value assesses the statistical significance of the difference between the conditional probability of the IV and the margins. This ratio will be used as part of the selection criteria for selecting important IV states in the model, as discussed in the next section, Results.

Risk ratios help explain effect size, as does the reduction of uncertainty $(\Delta \mathrm{H})$, but provide a different way to look at the effect. For $\Delta \mathrm{H}$, even small uncertainty reductions could be large in effect size (like $1: 1$ to $2: 1$ ). The measure $\Delta \mathrm{H}$ is like \%variance explained, with the major exception that low \%variance explained means the effect size is ignorable. However, for $\Delta \mathrm{H}$, even small numbers can have large effect sizes (this is because there is a log). The following example is used to help explain what the risk ratio means. This is important because the risk ratio is the primary measure by which decreased or increased risk is assessed.

In this example, the season can be either summer or winter, and the possibilities for weather can be either rain or no rain (Figure 3 below). If you do not know what season it is, then you face maximum uncertainty with a 1:1 chance of no-rain to rain. However, if you know the season, the uncertainty is reduced. If you know it is winter, then there is a 1:2 odds of no rain:rain. If you know it is summer, then there is a 2:1 odds of no rain:rain. Knowing the season changes the odds. This is a big effect size and correlates to a $\% \Delta \mathrm{H}$ of 8 . 


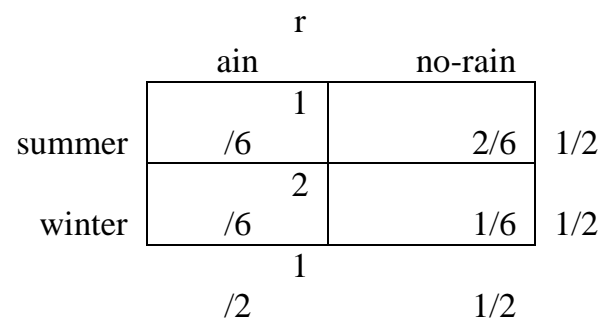

Figure 3. Knowing the Season Reduces the Uncertainty of the No-Rain:Rain from 1:1 to 2:1 or 1:2 odds.

In a decision tree with probabilities, this would look like Figure 4.

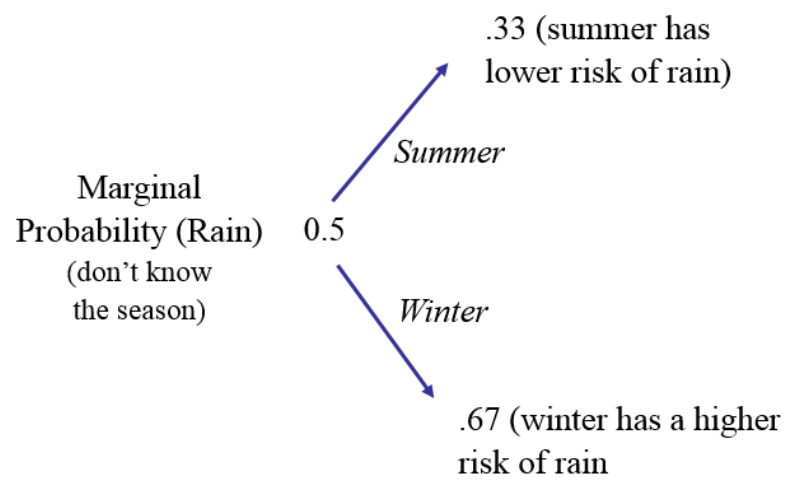

Figure 4. Decision Tree Illustrating How Knowing the Season Reduces the Uncertainty of the No-Rain:Rain from 1:1 to 2:1 or 1:2 Odds.

In this example, the risk ratio is the probability of an outcome (e.g., rain) for a particular IV state (e.g., summer) divided by the marginal probability of the outcome (maximum uncertainty):

$$
\frac{.33}{.5} \text { or } \frac{.67}{.5}
$$

For the three binary DVs (Complication, SNF, and Readmission), the risk ratio is the probability of an outcome for a particular adverse IV state divided by the marginal probability of this outcome for the whole sample. For the DV Total Cost, however, the 
ratio is instead the Expected Value of an IV state divided by the average cost for the total sample.

Trying out many models in an exploratory modeling approach can lead to false positives, and it is valuable to try to guard against this. In this project, decisions were made that were systematically conservative by (a) dropping Comorbidity IVs that were infrequent in the data, (b) requiring a frequency $\geq 10$ for each of the IV states in the model, (c) using $\triangle \mathrm{BIC}$ as model selection criterion, and (d) establishing the effect size of at least $10 \%$ in the Risk Ratio of IV states.

The general LR and RA methodologies were described in the Literature Review chapter, and project-specific applications and extensions of these methods are described above in this Methodology chapter. In the following chapter, the LR and RA analysis is performed, and the results are presented. 


\section{Chapter 4. Results}

In the last chapter, an overview of the data and methods was provided. In this chapter, the first research objective aims to connect this project to the literature, and then compare Logistic Regression (LR) and Reconstructability Analysis (RA) in both confirmatory and exploratory modes. The second research question aims to find predictive models with RA. This chapter describes the analyses and presents the results in detail. These results are then summarized in the following Discussion chapter.

\section{Preliminary Research Objective: LR \& RA Comparison}

To establish a connection to results from the literature, results are presented from an LR analysis of this project's data, using a limited set of variables. Then, to address the question whether RA and LR give the same results for equivalent models, results are presented for the comparison of RA to LR using the same limited set of variables. To answer the question whether exploratory RA can provide better or novel models compared to LR, results are presented from an exploratory RA analysis, first on the limited data set and then on a larger subset of variables. This larger subset of variables is also analyzed with stepwise LR for comparison. These analyses provide a sequence of (limited) connections from LR results in the literature, to LR results from this project's data, to RA results on this project's data.

\section{Connecting to Previous LR Results from the Literature}

Prior to LR and RA comparisons, the results of a previously published study that used LR was re-created, also using LR, on this project's data. The re-creation of this past 
study confirmed that this data set was comparable to the data set used in that study, thus validating this project's data.

Previous research has assessed the effect of comorbidities (hypertension $(\mathrm{H})$, diabetes (D), obesity (O), and their combinations) on postoperative complications and non-homebound discharge for patients with hip and knee arthroplasty (Jain et al., 2005). In the Jain study, LR was used to determine that postoperative complications were more likely in patients with hypertension $(\mathrm{H})$, diabetes $(\mathrm{D})$, or obesity $(\mathrm{O})$ as compared with patients without these individual comorbidities. Jain et al, used the large National Inpatient Sample (NIS) database to create a data set of over a million joint replacement cases - a much larger sample than the data set used in this project. However, the procedure and diagnosis coding methodology and the way DVs postoperative complications and non-homebound discharges were determined are identical. The similarity of patient cohort and variable definitions makes it possible to validate the data used in this project.

The hip and knee data sets used in this project were transformed into a combined data set $(n=6,612)$ similar to the data set used in the Jain study (Jain et al., 2005). The statistical software package R was used to perform LR and obtain odds ratios to quantify effect sizes and p-values to assess significance. Results demonstrate that odds ratios determined from this project's data are comparable to findings of the Jain study.

The Jain study (Jain et al., 2005) showed that hypertension had an effect on postoperative complications with an odds ratio (OR) of 1.07. Results of the new LR analysis (Table 10) show a slightly higher odds ratio at 1.18, although this result was not significant $(\mathrm{p}=0.18)$. Previous results showed that for patients with the comorbidity of 
obesity, there was a $31 \%$ greater chance of developing postoperative complications $(\mathrm{OR}=1.31)$, while in the new $\mathrm{LR}$ analysis an odds ratio of 1.39 was found with a significant p-value of 0.03 . Previous results indicate that diabetes increased risk of a postoperative complication by $6 \%$ (Jain et al., 2005), while patients with diabetes in this new analysis were 2.9 times more likely to have a postoperative complication than their non-diabetic counterparts $(p=0.00)$. The odds of diabetes are much higher in the new LR analysis than the previous results, yet the rate of diabetes is similar in both cohorts. There was a prevalence of diabetes of $10.04 \%$ in the Jain study cohort compared with $12.66 \%$ in the new LR analysis cohort.

Table 10. Previous Results Reported in (Jain et al., 2005), and Results from New LR Analysis on This Project's Data.

$\Delta \mathrm{df}, \Delta \mathrm{LR}$, and $\Delta \mathrm{AIC}$ values are given in the table for later comparisons with RA calculations.

\begin{tabular}{|c|c|c|c|c|c|c|c|c|}
\hline \multirow[b]{2}{*}{ Comorbidity (IV) } & \multirow[b]{2}{*}{ Outcome } & \multicolumn{2}{|c|}{$\begin{array}{c}\text { Previous Study } \\
\text { Results } \\
\end{array}$} & \multicolumn{5}{|c|}{ New (LR) } \\
\hline & & OR & $\mathrm{p}$-value & OR & $\mathrm{p}$-value & $\Delta \mathrm{df}$ & $\Delta \mathrm{LR}$ & $\triangle \mathrm{AIC}$ \\
\hline \multirow{2}{*}{ Hypertension } & $\begin{array}{l}\text { Postoperative } \\
\text { Complications }\end{array}$ & 1.07 & $<0.001$ & 1.18 & 0.18 & 1 & 1.78 & -0.22 \\
\hline & $\begin{array}{l}\text { Nonhomebound } \\
\text { Discharge }\end{array}$ & 1.12 & $<0.001$ & 1.07 & 0.17 & 1 & 1.85 & -0.15 \\
\hline \multirow{2}{*}{ Obesity } & $\begin{array}{l}\text { Postoperative } \\
\text { Complications }\end{array}$ & 1.31 & $<0.001$ & 1.39 & 0.03 & 1 & 4.48 & 2.48 \\
\hline & $\begin{array}{l}\text { Nonhomebound } \\
\text { Discharge }\end{array}$ & 1.45 & $<0.001$ & 1.22 & 0.00 & 1 & 9.38 & 7.38 \\
\hline \multirow{2}{*}{ Diabetes } & $\begin{array}{l}\text { Postoperative } \\
\text { Complications }\end{array}$ & 1.06 & 0.010 & 2.90 & 0.00 & 1 & 49.56 & 47.56 \\
\hline & $\begin{array}{l}\text { Nonhomebound } \\
\text { Discharge }\end{array}$ & 1.30 & $<0.001$ & 1.48 & 0.00 & 1 & 27.58 & 25.58 \\
\hline
\end{tabular}

The likelihood of a non-homebound discharge was $12 \%$ greater for patients with hypertension in the previous study $(\mathrm{OR}=1.12)$, and $7 \%$ greater in the new LR analysis $(\mathrm{p}=0.17)($ Table 10). Previous results showed that patients with diabetes had a $45 \%$ greater chance of a non-homebound discharge, while new LR results showed a $22 \%$ greater chance $(\mathrm{p}=0.00)$. Diabetes was a comorbidity that increased likelihood of a 
non-homebound discharge in both previous and current LR analysis, with a $30 \%$ greater chance $(\mathrm{OR}=1.30)$ in previous results compared to a $48 \%$ greater chance in the new $L R$ results. While there were differences in the effect sizes, the odds ratios were roughly comparable. The lack of significance in the effect size for hypertension on both DVs in the new LR analysis could be due to the much smaller cohort.

The previous study (Jain et al., 2005) also looked at patients who had combinations of two comorbidities. These were not hypothesized interaction terms to be analyzed, but rather three new variables created. Again, the new LR analysis showed comparable results in terms of odds ratios with most results being significant (Table 11).

Table 11. Previous Results Reported in (Jain et al., 2005) for 3 IVs, and Results from New LR Analysis on this Project's Data.

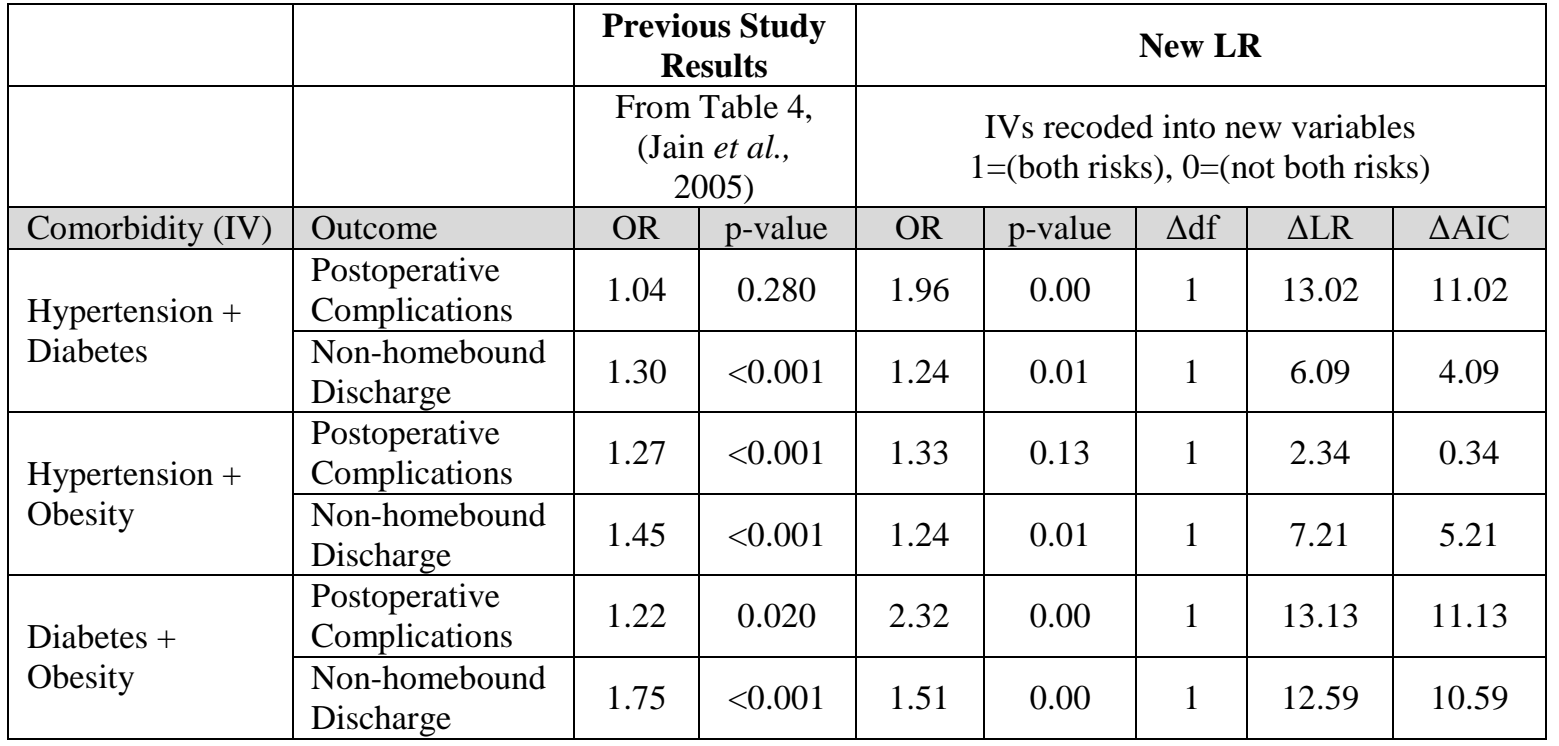

\section{Do $R A$ and LR give the same results for equivalent models?}

Results from the previous LR analysis were reproduced using the RA method instead. Here, RA was not used for exploratory modeling, but rather used in a confirmatory mode starting with the hypothesized model that was recreated in the LR 
analysis. In this comparison, RA as programmed in the software "Occam" was used in a confirmatory mode starting with the models used in the LR study. RA generated identical results to LR, demonstrating that where the methods overlap, they are equivalent. The $\triangle \mathrm{AIC}$ and Alpha measures are identical between LR and RA (Table 10 and Table 12, respectively).

Table 12. Similar RA Results Using this Project's Data.

\begin{tabular}{|l|l|c|c|c|c|c|c|}
\hline & & \multicolumn{7}{|c|}{ New Results (RA in "Occam") } \\
\hline Comorbidity (IV) & Outcome & OR & Alpha & $\% \Delta$ H(DV) & $\Delta$ df & $\Delta$ LR & $\Delta$ AIC \\
\hline \multirow{3}{*}{ Hypertension } & $\begin{array}{l}\text { Postoperative } \\
\text { Complications }\end{array}$ & 1.18 & 0.18 & 0.08 & 1 & 1.78 & -0.22 \\
\cline { 2 - 9 } & $\begin{array}{l}\text { Non-homebound } \\
\text { Discharge }\end{array}$ & 1.07 & 0.18 & 0.02 & 1 & 1.85 & -0.16 \\
\hline \multirow{3}{*}{ Obesity } & $\begin{array}{l}\text { Postoperative } \\
\text { Complications }\end{array}$ & 1.39 & 0.03 & 0.20 & 1 & 4.48 & 2.48 \\
\cline { 2 - 9 } & $\begin{array}{l}\text { Non-homebound } \\
\text { Discharge }\end{array}$ & 1.22 & 0.00 & 0.10 & 1 & 9.38 & 7.38 \\
\hline \multirow{2}{*}{ Diabetes } & $\begin{array}{l}\text { Postoperative } \\
\text { Complications }\end{array}$ & 2.90 & 0.00 & 2.19 & 1 & 49.56 & 47.56 \\
\cline { 2 - 8 } & $\begin{array}{l}\text { Non-homebound } \\
\text { Discharge }\end{array}$ & 1.48 & 0.00 & 0.30 & 1 & 27.58 & 25.58 \\
\hline
\end{tabular}

Results from RA are summarized as conditional probability distributions, which were transformed into joint probability distributions from which the odds ratios were calculated. The odds ratios are identical to those calculated by LR. RA provides an additional measure of effect size in the percent reduction of uncertainty of the dependent variable given the comorbidity IV states: $\% \Delta \mathrm{H}(\mathrm{DV})$, as seen in Table 12 . This reduction of uncertainty is a unique and central feature in the RA methodology and will be emphasized in the upcoming exploratory modeling section. It might be viewed as a way of summarizing several odds ratios in a single measure. Uncertainty is the nominal variable "equivalent" of variance, and so a \%reduction of uncertainty resembles a \%variance explained. (For Gaussian distributions, there is in fact an equation that directly 
relates the two.) However, uncertainty reduction numbers that are small can still represent large effect sizes, because of the logarithm term in the expression for uncertainty (Shannon entropy).

Using RA to generate results for patients who had two comorbidities again resulted in identical $\triangle \mathrm{AIC}$, alpha, and odds ratio numbers (Table 13). However, the $\% \Delta \mathrm{H}(\mathrm{DV})$ is rather low, with the largest reduction in uncertainty at $0.57 \%$. Perhaps there were other IVs that would have provided more information about the DV. The fact that even low reductions of uncertainty can correspond to odds ratio values whose difference from 1 is statistically significant should be kept in mind when uncertainty reduction values are reported in the next section.

Table 13. Similar RA Results Using this Project's Data with 3 New IVs.

\begin{tabular}{|l|l|c|c|c|c|c|c|}
\hline & & \multicolumn{7}{|c|}{ New Results (RA) } \\
\hline Comorbidity (IV) & Outcome & OR & Alpha & $\% \Delta \mathrm{H}(\mathrm{DV})$ & $\Delta \mathrm{df}$ & $\Delta \mathrm{LR}$ & $\Delta \mathrm{AIC}$ \\
\hline \multirow{2}{*}{$\begin{array}{l}\text { Hypertension + } \\
\text { Diabetes }\end{array}$} & $\begin{array}{l}\text { Postoperative } \\
\text { Complications }\end{array}$ & 1.96 & 0.00 & 0.57 & 1 & 13.02 & 11.02 \\
\cline { 2 - 9 } & $\begin{array}{l}\text { Non-homebound } \\
\text { Discharge }\end{array}$ & 1.24 & 0.01 & 0.07 & 1 & 6.09 & 4.09 \\
\hline \multirow{2}{*}{$\begin{array}{l}\text { Hypertension + } \\
\text { Obesity }\end{array}$} & $\begin{array}{l}\text { Postoperative } \\
\text { Complications }\end{array}$ & 1.33 & 0.13 & 0.10 & 1 & 2.34 & 0.34 \\
\cline { 2 - 9 } & $\begin{array}{l}\text { Non-homebound } \\
\text { Discharge }\end{array}$ & 1.24 & 0.01 & 0.08 & 1 & 7.21 & 5.21 \\
\hline \multirow{2}{*}{$\begin{array}{l}\text { Diabetes + } \\
\text { Obesity }\end{array}$} & $\begin{array}{l}\text { Postoperative } \\
\text { Complications }\end{array}$ & 2.32 & 0.00 & 0.58 & 1 & 13.13 & 11.13 \\
\cline { 2 - 8 } & $\begin{array}{l}\text { Non-homebound } \\
\text { Discharge }\end{array}$ & 1.51 & 0.00 & 0.14 & 1 & 12.59 & 10.59 \\
\hline
\end{tabular}

\section{Does exploratory RA provide better or novel models compared to LR?}

This preliminary comparison was then expanded, and RA was then used in an exploratory mode, providing an initial example of the type of unique results that are possible from using RA. This question is first answered using a simple RA exploratory search using the limited variable set from the above LR and RA analysis. Then, to further 
demonstrate RA's exploratory search capabilities, a larger subset of the data with 17 IVs was analyzed with LR and RA.

Using this simplified data set with only four variables - the IVs, hypertension $(\mathrm{H})$, diabetes (D), and obesity (O), and the DV, complications (C), shows a simple example of RA exploratory modeling, which in this case can evaluate the complete set of all possible models. In Table 14 below, the bottom row shows the independence model, where there is no relation or constraint between the IVs and the DVs with zero reduced DV uncertainty $(\% \Delta \mathrm{H}(\mathrm{DV})=0.00)$. Moving up the table, or up the lattice of structures of all possible models for this four-variable system, the top row shows the data, the "saturated model," which has information $=1$ and maximum complexity $(\Delta \mathrm{df}=7)$. The models in between independence and the data were all considered by RA. While by the AIC criterion the best model was determined to be IV:HDC, there was an opportunity to consider models with loops, such as those highlighted in orange in Table 14 below. In this simple analysis, the model chosen did not have a loop, but in other situations, a model with loops may be the best model by AIC or another criterion. Note that IV:DC has information of 0.81 , and adding $\mathrm{HC}$ (to give model IV:DC:HC) does not increase this value, but going up and adding a genuine interaction effect (to give model IV:HDC) increases the information to 0.98 . Note also that although $\mathrm{O}$ predicts $\mathrm{C}$ better than $\mathrm{H}$ predicts $\mathrm{C}$, the $\mathrm{HD}$ interaction predicts $\mathrm{C}$ better (information $=0.98$, uncertainty reduction $=2.67 \%$ ) than the DO interaction (information $=0.83$, uncertainty reduction $=2.24 \%$ ) 
Table 14. Directed RA Search for DV Complication for 4 Variables.

(Best Model by AIC in Bold.)

\begin{tabular}{|l|c|c|c|c|c|c|c|}
\hline MODEL & Level & $\Delta$ df & $\Delta$ LR & Inf & $\% \Delta H(D V)$ & $\Delta$ AIC & Alpha \\
\hline HDOC (Data) & 7 & 7 & 61.44 & 1.00 & 2.71 & 47.44 & 0.00 \\
\hline IV:HDC:HOC:DOC & 6 & 6 & 61.12 & 0.99 & 2.69 & 49.12 & 0.00 \\
\hline IV:HDC:DOC & 5 & 5 & 61.11 & 0.99 & 2.69 & 51.11 & 0.00 \\
\hline IV:HDC:OC & 4 & 4 & 60.80 & 0.99 & 2.68 & 52.80 & 0.00 \\
\hline IV:HDC:HOC & 5 & 5 & 60.80 & 0.99 & 2.68 & 50.80 & 0.00 \\
\hline IV:HDC & $\mathbf{3}$ & $\mathbf{3}$ & $\mathbf{6 0 . 4 7}$ & $\mathbf{0 . 9 8}$ & $\mathbf{2 . 6 7}$ & $\mathbf{5 4 . 4 7}$ & $\mathbf{0 . 0 0}$ \\
\hline IV:HOC:DOC & 5 & 5 & 51.29 & 0.83 & 2.26 & 41.29 & 0.00 \\
\hline IV:HC:DOC & 4 & 4 & 50.89 & 0.83 & 2.24 & 42.89 & 0.00 \\
\hline IV:DOC & 3 & 3 & 50.85 & 0.83 & 2.24 & 44.85 & 0.00 \\
\hline IV:HOC:DC & 4 & 4 & 50.48 & 0.82 & 2.23 & 42.48 & 0.00 \\
\hline IV:HC:DC:OC & 3 & 3 & 49.88 & 0.81 & 2.20 & 43.88 & 0.00 \\
\hline IV:DC:OC & 2 & 2 & 49.82 & 0.81 & 2.20 & 45.82 & 0.00 \\
\hline IV:HC:DC & 2 & 2 & 49.57 & 0.81 & 2.19 & 45.57 & 0.00 \\
\hline IV:DC & 1 & 1 & 49.56 & 0.81 & 2.18 & 47.56 & 0.00 \\
\hline IV:HOC & 3 & 3 & 5.83 & 0.09 & 0.26 & -0.17 & 0.12 \\
\hline IV:HC:OC & 2 & 2 & 5.37 & 0.09 & 0.24 & 1.37 & 0.07 \\
\hline IV:OC & 1 & 1 & 4.48 & 0.07 & 0.20 & 2.48 & 0.03 \\
\hline IV:HC & 1 & 1 & 1.78 & 0.03 & 0.08 & -0.22 & 0.18 \\
\hline IV:C (Independence model) & 0 & 0 & 0.00 & 0.00 & 0.00 & 0.00 & 1.00 \\
\hline MODEL & Level & $\Delta$ df & $\Delta$ LR & Inf & $\% \Delta H(D V)$ & $\Delta$ AIC & Alpha \\
\hline
\end{tabular}

\section{RA Exploratory and Stepwise LR}

This previous example illustrated a very simple comparison where LR had been used in its most standard form. In the next example, an exploratory "stepwise" approach was used that illustrates a more typical research approach. This stepwise LR approach is more comparable than confirmatory modeling to the exploratory modeling using RA.

In this example, the literature was surveyed in order to select a set of variables that have been reported to have an effect on outcomes similar to those in this study. The resulting 17 literature-based predictors became the IVs used in this analysis. With these 17 IVs, LR (as implemented in R) was then used in both its regular and stepwise variations. Without any additional hypothesis, the best models using LR are presented. RA as implemented in "Occam" is then used with the same 17 IVs. RA looked at models 
not considered in the LR stepwise approach. The models from each method are compared and discussed.

In the first example, 17 Comorbidity IVs were selected that have been found to be potentially predictive of cost from the literature, and were included in the hip or knee data sets used in the RA exploratory searches. This example analysis was performed on the hip data set for the DV Total Cost (Tcb). While total cost was binned into three states for RA exploratory modeling in the next results section, Tcb is binned into two states in order to perform a simple LR comparison. (LR can be used with the DV having more than two states, but the analysis is cumbersome.)

\section{- Single IV Predictors}

First, a single predicting search was performed with the 17 IVs specified. As is seen in Table 15, RA and LR produced identical results. In this single predicting search, six IVs were individually predictive with $\mathrm{p}<0.05$. 
Table 15. LR \& RA Comparison of Results for Single Predicting Search. $(\Delta d f=1$ for every model in this table.)

\begin{tabular}{|c|c|c|c|c|c|c|c|c|c|}
\hline \multicolumn{4}{|c|}{ Logistic Regression Results } & \multicolumn{6}{|c|}{ RA Results } \\
\hline MODEL & $\triangle \mathrm{AIC}$ & $\Delta \mathrm{LR}$ & Alpha & MODEL & $\triangle \mathrm{AIC}$ & $\% \Delta \mathrm{H}$ & $\Delta \mathrm{LR}$ & $\% \mathrm{C}$ & Alpha \\
\hline $\begin{array}{l}17 \mathrm{IVs} \\
\text { (single } \\
\text { predicting) }\end{array}$ & & & & $\begin{array}{l}17 \mathrm{IVs} \\
\text { (single } \\
\text { predicting) }\end{array}$ & & & & & \\
\hline Rmo Tcb & 47.11 & 49.11 & 0.00 & Rmo Tcb & 47.11 & 1.11 & 49.11 & 52.48 & 0.00 \\
\hline Rdi Tcb & 8.19 & 10.19 & 0.00 & Rdi Tcb & 8.19 & 0.23 & 10.19 & 51.64 & 0.00 \\
\hline Rhf Tcb & 5.07 & 7.07 & 0.01 & Rhf Tcb & 5.07 & 0.16 & 7.07 & $\mathbf{5 0 . 5 8}$ & 0.01 \\
\hline Rdh Tcb & 4.94 & 6.94 & 0.01 & Rdh Tcb & 4.94 & 0.16 & 6.94 & 50.14 & 0.01 \\
\hline Rao Tcb & 2.81 & 4.81 & 0.03 & Rao Tcb & 2.81 & 0.11 & 4.81 & 50.61 & 0.03 \\
\hline Rua Tcb & 2.75 & 4.75 & $\mathbf{0 . 0 3}$ & Rua Tcb & 2.75 & 0.11 & 4.75 & 50.92 & 0.03 \\
\hline Roo Tcb & 1.46 & 3.46 & 0.06 & Roo Tcb & 1.46 & 0.08 & 3.46 & 50.92 & 0.06 \\
\hline Rpv Tcb & 0.52 & 2.52 & 0.11 & Rpv Tcb & 0.52 & 0.06 & 2.52 & 50.23 & 0.11 \\
\hline Rhe Tcb & 0.38 & 2.38 & 0.12 & Rhe Tcb & 0.38 & 0.05 & 2.38 & 51.36 & 0.12 \\
\hline Rci Tcb & 0.24 & 2.24 & 0.13 & Rci Tcb & 0.24 & 0.05 & 2.24 & 50.42 & 0.13 \\
\hline Rov Tcb & -0.30 & 1.70 & 0.19 & Rov Tcb & -0.30 & 0.04 & 1.70 & 50.14 & 0.19 \\
\hline Rbe Tcb & -0.35 & 1.65 & 0.20 & Rbe Tcb & -0.35 & 0.04 & 1.65 & 50.11 & 0.20 \\
\hline Rco Tcb & -0.70 & 1.30 & 0.25 & Rco Tcb & -0.70 & 0.03 & 1.30 & 50.17 & 0.25 \\
\hline Rca Tcb & -1.09 & 0.91 & 0.34 & Rca Tcb & -1.09 & 0.02 & 0.91 & 50.36 & 0.34 \\
\hline Rem Tcb & -1.10 & 0.90 & 0.34 & Rem Tcb & -1.10 & 0.02 & 0.90 & 50.11 & 0.34 \\
\hline Rdn Tcb & -1.30 & 0.70 & 0.40 & Rdn Tcb & -1.30 & 0.02 & 0.70 & 50.08 & 0.40 \\
\hline Rpe Tcb & -1.91 & 0.09 & 0.76 & Rpe Tcb & -1.91 & 0.00 & 0.09 & 50.02 & 0.76 \\
\hline
\end{tabular}

Looking at all 17 of the IVs together results in a $\triangle \mathrm{AIC}$ of 51.85 , as seen in

Table 16. Looking at a model that includes only the six IVs that were individually predictive of total cost with alpha $<0.05$ resulted in the LR model Rmo Tcb : Rdi Tcb :

Rhf Tcb : Rdh Tcb : Rao Tcb : Rua Tcb with a $\triangle$ AIC of 60.51 (Table 16), which is an improvement over the model with all 17 IVs.

Table 16. Confirmatory LR Results for all 17 IVs and 6 IVs with $\mathbf{p}<0.05$.

\begin{tabular}{|l|c|c|c|c|c|}
\hline Model & $\Delta$ df & $\Delta$ AIC & $\Delta$ LR & Alpha \\
\hline All 17 variables & 17 & 51.85 & 85.85 & 0.00 \\
\hline \multicolumn{5}{|l|}{} \\
\hline Model & $\Delta$ df & $\Delta$ AIC & $\Delta$ LR & Alpha \\
\hline Model with the 6 IVs variables that individually have $p<0.05$ & $\mathbf{6}$ & $\mathbf{6 0 . 5 1}$ & $\mathbf{7 2 . 5 1}$ & $\mathbf{0 . 0 0}$ \\
\hline Rmo Tcb : Rdi Tcb : Rhf Tcb : Rdh Tcb : Rao Tcb : Rua Tcb & $\mathbf{6}$
\end{tabular}


Using the same 17 IVs, a stepwise LR approach yielded model Rdi Tcb : Roo

Tcb : Rmo Tcb : Rov Tcb : Rhf Tcb : Rdh Tcb : Rua Tcb : Rao Tcb with a $\triangle$ AIC of

62.66 (Table 17), an improvement over the previous best LR model from Table 16.

Table 17. Stepwise LR Approach with 17 IVs.

\begin{tabular}{|l|c|c|c|c|}
\hline Model & $\Delta$ df & $\Delta$ AIC & $\Delta$ LR & Alpha \\
\hline Best model from a stepwise search using AIC & $\mathbf{8}$ & $\mathbf{6 2 . 6 6}$ & $\mathbf{7 8 . 6 6}$ & $\mathbf{0 . 0 0}$ \\
\hline $\begin{array}{l}\text { Rdi Tcb: Roo Tcb: Rmo Tcb : Rov Tcb : Rhf Tcb : Rdh Tcb : } \\
\text { Rua Tcb : Rao Tcb }\end{array}$
\end{tabular}

While the researcher must specify interaction terms for an LR analysis, even with 17 IVs, RA (as implemented in Occam) automatically considers these models in its standard search. In addition, RA considers models that include multivariate interaction effects that are not possible with LR. The best fine-grained model by $\triangle \mathrm{AIC}$ in the 17-variable search using RA was Rd Rao Tcb : Roo Rpv Tcb : Rmo Rci Tcb : Rmo Rao

Tcb : Rov Rua Tcb : Rhf Rua Tcb : Rdh Tcb with a $\Delta$ AIC of 66.57 (Table 18), an improvement over the model from the LR stepwise search in Table 17.

Table 18. RA Exploratory Search Results with 17 IVs (no interaction terms specified in advance).

\begin{tabular}{|c|c|c|c|c|c|c|c|}
\hline MODEL & $\Delta \mathrm{df}$ & $\triangle \mathrm{AIC}$ & $\% \Delta \mathrm{H}$ & $\Delta \mathrm{LR}$ & $\% \mathrm{C}$ & alpha & Variable description \\
\hline \multicolumn{8}{|c|}{ FINE, best models (with loops) } \\
\hline \multicolumn{8}{|c|}{$\Delta \mathrm{AIC}$ (best model) } \\
\hline $\begin{array}{c}\text { RdiRaoTcb : } \\
\text { RooRpvTcb : } \\
\text { RmoRci Tcb : } \\
\text { RmoRaoTcb : } \\
\text { RovRuaTcb : } \\
\text { RhfRuaTcb : } \\
\text { RdhTcb }\end{array}$ & 16 & 66.57 & 2.22 & 98.57 & 55.69 & 0.08 & $\begin{array}{l}\text { Diabetes mellitus (RISK 250) + Chronic } \\
\text { airway obstruction, not elsewhere } \\
\text { classified (RISK 496), Overweight, } \\
\text { obesity and other hyperalimentation } \\
\text { (RISK 278) + Peripheral vascular } \\
\text { disease, unspecified (RISK 443.9), } \\
\text { Morbid obesity (RISK 278.01) + Other } \\
\text { forms of chronic ischemic heart disease } \\
\text { (RISK 414), Morbid obesity (RISK } \\
\text { 278.01) + Chronic airway obstruction, } \\
\text { not elsewhere classified (RISK 496), } \\
\text { Overweight (RISK 278.02) + Asthma } \\
\text { unspecified (RISK 493.9), Heart failure } \\
\text { (RISK 428) + Asthma unspecified } \\
\text { (RISK 493.9), Diastolic heart failure } \\
\text { (RISK 428.3) }\end{array}$ \\
\hline
\end{tabular}


RA has previously been used by several researchers to detect interaction terms that are then specified for LR analysis (Cangur, 2009; Carletti, 2004; Mist, 2007). The RA analysis detected interaction effects and in fact, each predicting component in the best model by AIC from the RA search had an interaction term (Table 18). In this analysis, the RA exploratory search appears to be more capable of finding predictive interactions. When using the $10 \mathrm{IVs}$ (out of the original $17 \mathrm{IVs}$ ) that are present in the best model from the RA search (with no interaction terms), an LR analysis resulted in a $\triangle \mathrm{AIC}$ of 61.67 (Table 19). However, RA found a more interesting and informative $(\triangle \mathrm{AIC}=66.57)$ model that LR could not find, because RA automatically searches for significant interaction effects.

Table 19. LR Analysis with the 10 IVs present in the Best RA Model.

\begin{tabular}{|l|c|c|c|c|}
\hline Model & $\Delta$ df & $\Delta$ AIC & $\Delta$ LR & Alpha \\
\hline Model with 10 variables that are present in Occam's "best model by AIC" & 10 & 61.67 & 81.67 & 0.00 \\
\hline $\begin{array}{l}\text { Rdi Tcb : Rao Tcb : Roo Tcb : Rpv Tcb : Rmo Tcb : Rci Tcb : } \\
\text { Rov Tcb : Rua Tcb : Rhf Tcb : Rdh Tcb }\end{array}$ & & &
\end{tabular}

In this example looking at just 17 IVs, RA generated models with quantifiable additional predictive power by considering models that were not considered in the LR analysis. Note that RA and LR looked at the same set of IVs. The higher AIC value for the RA model means that its additional complexity (the RA model in Table 18 is twice as complex as the LR model in Table 17) is more than justified by the increase in predictive efficacy that it gives.

In the next section, a much larger set of IVs is considered when looking at each of the dependent variables of this study. In addition to the previously known predicting IVs, RA may detect something novel, particularly through the form of an interaction term. 
These combination effects may add predictive strength relative to the set of known single predicting IVs available in the current literature and may even detect surprising IVs.

The first example in this preliminary comparison section recreated a previous study and addressed any concern of whether RA is approximately similar to LR where they overlap methodologically. This validated RA as a method and confirmed the results from a previous LR study. The second example showed that RA can provide novel predictions and better relative performance, and therefore RA was shown to have value as a method to augment or supplant LR.

\section{Main Research Objective: Find predictive models with RA}

In this results section, a series of best models whose measure of goodness is

$\%$ reduction of uncertainty of the DV are proposed and analyzed in detail for the following DVs: (a) Complication, (b) (discharge to) Skilled Nursing Facility, (c) Readmission, and (d) Total Cost, for both Knee and Hip replacement data. In each of these eight studies, searches were performed looking at (a) All IVs together, and (b) only the Comorbidity IVs.

\section{DV: Complication (Cp)}

Knee Analysis

- All IVs

- Coarse Searches (Models without Loops)

First, loopless models were examined for the dependent variable Complication (Cp). These loopless models were sorted from the most predictive to the least predictive, 
and models were selected with single IV predictors having $\mathrm{p} \leq 0.05$, which resulted in marking 53 IVs to keep for the next round of searches. In addition to these single predicting variables, IVs were retained that were found to be predictive in the literature on similar outcome variables. In this literature, there were 25 variables that were predictive of complications or discharge destination that were retained in the data; 16 of these literature-based IVs also had a p value $<0.05$ in the single predicting search, while nine of the literature-based variables had $p$ values $>0.05$. The search results for the top 10 models are included in Table 20. Additional single predicting IVs are provided in Table 20 if the IV was not listed in the top 10 but was included in one of the best models by the BIC or AIC criterion. In results tables where $\mathrm{p}$ values always equaled zero to two significant figures (i.e., $\mathrm{p}<0.005)$, the column indicating $\mathrm{p}$ value was omitted. A model in the table specifies IVs (e.g., Nrb, Rku) that predict the DV (here, Cp). It is followed first by $\Delta \mathrm{df}=\mathrm{df}($ model $)-\mathrm{df}($ reference $)$, the difference between the degrees of freedom of the model and the reference or independence model. The next value is $\Delta \mathrm{BIC}=\mathrm{BIC}($ reference $)-\mathrm{BIC}($ model $)$, for which improvements in the model compared to the reference are reflected in larger values. The next measurement is $\% \Delta H=H(D V)-$ $\mathrm{H}(\mathrm{DV} \mid \mathrm{IV})$, the \%reduction of uncertainty of the DV given the IVs. The reduction of uncertainty measure indicates how predictive the IVs are. The BIC measure indicates how efficient the prediction is; i.e., how predictive the IVs are, given their complexity (degrees of freedom). Best models are chosen based on their BIC measures, which results in a highly conservative choice of models. Table 18 summarizes the results of single and multiple predictors in loopless and all-model (with loops) searches. 
Table 20. Summary of Search Results (Knee) for All IVs.

Search covers coarse and fine models. All $\mathbf{p}$-values $=0$.

\begin{tabular}{|c|c|c|c|c|}
\hline MODEL & $\Delta \mathrm{df}$ & $\Delta \mathrm{BIC}$ & $\% \Delta \mathrm{H}$ & Variable description \\
\hline \multicolumn{5}{|c|}{ COARSE, single predictors (top 10) } \\
\hline $\mathrm{S} \mathrm{Cp}$ & 62 & -412.74 & 6.45 & Surgeon \\
\hline Nrb Cp & 2 & 77.29 & 5.69 & Number of risks (binned) \\
\hline Rrd Cp & 1 & 43.04 & 3.11 & Unspecified hypertensive renal disease (403.9) \\
\hline Rku Cp & 1 & 39.63 & 2.91 & Chronic kidney disease, unspecified (585.9) \\
\hline Ruh Cp & 1 & 33.56 & 2.54 & Other and unspecified hyperlipidemia (272.4) \\
\hline $\mathrm{L}$ Cp & 6 & -9.04 & 2.50 & Location \\
\hline Ad Cp & 27 & -185.33 & 2.47 & Admission diagnosis \\
\hline Ageb Cp & 2 & 14.61 & 1.90 & Age (binned) \\
\hline Raf Cp & 1 & 11.46 & 1.20 & Atrial fibrillation (427.31) \\
\hline $\mathrm{Rhf} \mathrm{Cp}$ & 1 & 10.79 & 1.16 & Heart failure (428) \\
\hline MODEL & $\Delta \mathrm{df}$ & $\triangle \mathrm{BIC}$ & $\% \Delta \mathrm{H}$ & Variable description \\
\hline \multicolumn{5}{|c|}{ COARSE, IVs in AIC or BIC models but not in top 10} \\
\hline Rhd Cp (rank 12) & 1 & 9.90 & 1.11 & Other chronic pulmonary heart disease (416.8) \\
\hline Rro Cp (rank 18) & 1 & 3.22 & 0.70 & Rosacea (695.3) \\
\hline Reg Cp (rank 20) & 1 & 1.95 & 0.63 & Esophagitis (530.1) \\
\hline MODEL & $\Delta \mathrm{df}$ & $\triangle \mathrm{BIC}$ & $\% \Delta \mathrm{H}$ & Variable description \\
\hline \multicolumn{5}{|c|}{ COARSE, best model (loopless) } \\
\hline \multicolumn{5}{|c|}{$\Delta \mathrm{BIC}$ (best model) } \\
\hline Nrb Rku Cp & 5 & 83.23 & 7.58 & $\begin{array}{l}\text { Number of risks (binned), Chronic kidney disease, } \\
\text { unspecified (585.9) }\end{array}$ \\
\hline \multicolumn{5}{|c|}{ Inc.P \& $\triangle$ AIC (same best model) } \\
\hline Nrb Rhd Rku Cp & 11 & 52.71 & 8.77 & $\begin{array}{l}\text { Number of risks (binned), Other chronic pulmonary heart } \\
\text { disease (416.8), Chronic kidney disease (585.9) }\end{array}$ \\
\hline MODEL & $\Delta \mathrm{df}$ & $\triangle \mathrm{BIC}$ & $\% \Delta \mathrm{H}$ & Variable description \\
\hline \multicolumn{5}{|c|}{ FINE, best models (with loops) } \\
\hline \multicolumn{5}{|c|}{$\Delta \mathrm{BIC}$ (best model) } \\
\hline $\begin{array}{l}\text { Ageb Cp : Nrb Cp : } \\
\text { Ruh Cp:Rhd Cp : } \\
\text { Rku Cp : Rro Cp }\end{array}$ & 8 & 104.71 & 10.40 & $\begin{array}{l}\text { Age (binned), Number of risks (binned), Other and } \\
\text { unspecified hyperlipidemia (272.4), Other chronic } \\
\text { pulmonary heart disease (416.8), Chronic kidney disease, } \\
\text { unspecified (585.9), Rosacea (695.3) }\end{array}$ \\
\hline \multicolumn{5}{|c|}{ Inc.P \& $\triangle$ AIC (same best model) } \\
\hline $\begin{array}{l}\text { Ageb Cp : Nrb Cp : } \\
\text { Ruh Cp: Rhd Cp : } \\
\text { Reg Cp : Rku Cp : } \\
\text { Rro Cp }\end{array}$ & 9 & 104.23 & 10.88 & $\begin{array}{l}\text { Age (binned), Number of risks (binned), Other and } \\
\text { unspecified hyperlipidemia (272.4), Other chronic } \\
\text { pulmonary heart disease (416.8), Esophagitis (530.1), } \\
\text { Chronic kidney disease, unspecified (585.9), Rosacea } \\
\text { (695.3) }\end{array}$ \\
\hline
\end{tabular}

Knowing the surgeon who performed the surgery $(\mathrm{S})$ reduces uncertainty by $6.45 \%$ (Table 20). Likewise, knowing only if the patient had unspecified hypertensive renal disease (Rrd) reduces uncertainty by $3.11 \%$, and knowing that the patient had unspecified chronic kidney disease ( $\mathrm{Rku}$ ) reduces uncertainty by $2.91 \%$. The best coarse 
model in Table 20 shows that, for this data set, simply knowing the total number of comorbidities a patient had ( $\mathrm{Nrb})$ along with chronic kidney disease $(\mathrm{Rku})$ reduces the uncertainty in predicting if Complication (Cp) occurred by $7.58 \%$.

\section{- Fine Searches (Models with Loops)}

The next type of search considers models with loops, which allows for multiple components that predict the DV. Within each component, there may be interaction effects among the IVs in their prediction of the DV, just as an interaction effect was observed in the best BIC model, Nrb Rku Cp, and the best loopless IncrP/AIC model, Nrb Rhd Rku Cp, as shown in Table 20.

Note that some single predicting variables do not show up in the best coarse or fine models, indicating that the IVs are not independent from each other. There are six single predicting variables in the best BIC fine-grained model: Ageb Cp : Nrb Cp : Ruh Cp : Rhd Cp : Rku Cp : Rro Cp. Five of these variables-Ageb, Nrb, Ruh, Rhd, and $\mathrm{Rku}$ - also appear in the top 10 single predicting components, while Rro is the 18 th in the list of single predicting components (Appendix C). This apparently low-value variable was included when the RA search methodology sought to improve a model already containing the better individual predictors Ageb, Nrb, Ruh, Rhd, and Rku. Rro was found to be the variable that added more additional information to that model, relative to any of the better singly-predicting IVs above it.

The best single predictor, S (surgeon) does not appear in the best fine-grained model, presumably in part because $S$ has high cardinality and the information added by $S$ is not worth the complexity of including it in the model, and perhaps in part also because 
the predictive effect of $\mathrm{S}$ is already provided by the Ageb, Nrb, Ruh, Rhd, and/or Rku predictors. Similarly, Ageb, Nrb, Ruh, Rhd, and Rku contain the information offered by the other single predictors all the way down to Rro. The third-best single predictor, Rrd, also does not appear in the best fine-grained model. Again, the information added by Rrd is presumably not worth the additional complexity to be added to the model. This explanation is supported by the fact that if Rrd were the DV, it is well predicted by Ageb, Nrb, Ruh, Rhd, and Rku. In fact, Rku alone predicts Rrd with a \% $\Delta \mathrm{H}$ of $53.14 \%$, demonstrating significant overlap between Rku and Rrd. Nrb and other variables also explain information in Rrd, as seen in Table 21. This demonstrates the lack of independence between the IVs, which is analogous to collinearity among IVs in regression analysis.

Table 21. IV Rku Predicts Rrd (as DV), Demonstrating IV Overlap.

\begin{tabular}{|l|c|c|c|}
\hline MODEL & $\Delta \mathrm{df}$ & $\% \Delta \mathrm{H}(\mathrm{DV})$ & $\Delta \mathrm{BIC}$ \\
\hline Rku Rrd & 1 & 53.14 & 548.21 \\
\hline Rkd & 1 & 18.64 & 186.83 \\
\hline Rhe Rrd & 1 & 17.45 & 174.39 \\
\hline Nrb Rrd & 2 & 17.13 & 162.69 \\
\hline S Rrd & 62 & 11.42 & -399.60 \\
\hline Fc Rrd & 5 & 4.63 & 6.64 \\
\hline Ageb Rrd & 2 & 4.31 & 28.43 \\
\hline Rdi Rrd & 1 & 4.19 & 35.46 \\
\hline Ruh Rrd & 1 & 4.16 & 35.20 \\
\hline Ad Rrd & 27 & 3.43 & -190.21 \\
\hline
\end{tabular}

Recall that the IV Nrb is a binned variable that tallies up the number of comorbidities a patient has upon admission, and says nothing about the specific comorbidities and their effects. The Nrb IV is tallied based on the presence or absence of the 912 potential Comorbidity IVs present in the original data set. Because the specific effect of individual or interactive Comorbidities are of interest, the Comorbidity only 
search excludes $\mathrm{Nrb}$ and other All IVs and thus focuses explicitly on the potential effects of each individual comorbidity.

- Comorbidity IVs

- Coarse Searches (Models without Loops)

In the next search, Comorbidity IVs alone were considered as possible predictors. The two predicting All IVs [Number of Risks Binned (Nrb) and Age Binned (Ageb)] from Table 20 are thus not included in the results from this search (Table 22). The Comorbidities IVs from Table 20 also show up — in the same order as in Table 20 - as the most predictive single predictive IVs, and two additional single predicting IVs appear: aortic valve disorders (Rav), and coronary atherosclerosis of native coronary artery (Rca).

While the results of the search including both All IVs resulted in the best model Nrb Cp by the BIC criterion with a $\% \Delta \mathrm{H}$ of 6.73 (Table 20), the results of this search yielded a best model in which other and unspecified hyperlipidemia (Ruh) and unspecified hypertensive renal disease (Rrd) together predict Complication $(\mathbf{C p})$ with a $\% \Delta \mathrm{H}$ of 5.04 Table 22 below.

Table 22. Summary of Search Results (Knee) for Comorbidity IVs. Search covers directed coarse and fine models.

\begin{tabular}{|l|c|c|c|c|l|}
\hline MODEL & $\Delta$ df & $\Delta \mathrm{BIC}$ & $\% \Delta \mathrm{H}$ & Alpha & Variable description \\
\hline COARSE, single predictors & & & & & \\
\hline Rrd Cp & 1 & 43.04 & 3.11 & 0.00 & Unspecified hypertensive renal disease (403.9) \\
\hline Rku Cp & 1 & 39.63 & 2.91 & 0.00 & Chronic kidney disease, unspecified (585.9) \\
\hline Ruh Cp & 1 & 33.56 & 2.54 & 0.00 & Other and unspecified hyperlipidemia (272.4) \\
\hline Raf Cp & 1 & 11.46 & 1.20 & 0.00 & Atrial fibrillation (427.31) \\
\hline Rhf Cp & 1 & 10.79 & 1.16 & 0.00 & Heart failure (428) \\
\hline Rhd Cp & 1 & 9.90 & 1.11 & 0.00 & Other chronic pulmonary heart disease (416.8) \\
\hline Ros Cp & 1 & 9.81 & 1.10 & 0.00 & Obstructive sleep apnea (327.23) \\
\hline Rdi Cp & 1 & 8.45 & 1.02 & 0.00 & Diabetes mellitus (250) \\
\hline Rav Cp & 1 & 6.54 & 0.90 & 0.00 & Aortic valve disorders (RISK 424.1) \\
\hline
\end{tabular}




\begin{tabular}{|c|c|c|c|c|c|}
\hline MODEL & $\Delta \mathrm{df}$ & $\Delta \mathrm{BIC}$ & $\% \Delta \mathrm{H}$ & Alpha & Variable description \\
\hline Rca Cp & 1 & 6.48 & 0.90 & 0.00 & $\begin{array}{l}\text { Coronary atherosclerosis of native coronary } \\
\text { artery (RISK } 414.01 \text { ) }\end{array}$ \\
\hline MODEL & $\Delta \mathrm{df}$ & $\Delta \mathrm{BIC}$ & $\% \Delta \mathrm{H}$ & Alpha & Variable description \\
\hline \multicolumn{6}{|c|}{ COARSE, IVs in AIC or BIC models but not in top 10} \\
\hline Rro Cp (rank 18) & 1 & 3.22 & 0.70 & 0.00 & Rosacea (695.3) \\
\hline Rmo Cp (rank 19) & 1 & 2.72 & 0.67 & 0.00 & Morbid Obesity (278.01) \\
\hline $\operatorname{Reg} \mathrm{Cp}($ rank 20) & 1 & 1.95 & 0.63 & 0.00 & Esophagitis (530.1) \\
\hline Rlb Cp (rank 21) & 1 & 1.95 & 0.63 & 0.00 & Legal blindness (369.4) \\
\hline Rkd Cp (rank 22) & 1 & 1.84 & 0.62 & 0.00 & Chronic kidney disease, unspecified (585.9) \\
\hline Ruu Cp (rank 28) & 1 & -0.13 & 0.50 & 0.00 & $\begin{array}{l}\text { Other disorders of urethra and urinary tract } \\
(599)\end{array}$ \\
\hline Rpn Cp (rank 32) & 1 & -1.44 & 0.42 & 0.01 & $\begin{array}{l}\text { Unspecified hereditary and idiopathic periphera } \\
\text { neuropathy (356.9) }\end{array}$ \\
\hline Rcb Cp (rank 36) & 1 & -2.59 & 0.35 & 0.02 & Obstructive chronic bronchitis (491.2) \\
\hline Rpy Cp (rank41) & 1 & -3.11 & 0.32 & 0.02 & Polymyalgia rheumatica (725) \\
\hline Rhe Cp (rank42) & 1 & -3.14 & 0.32 & 0.02 & Unspecified essential hypertension (401.9) \\
\hline Rsy Cp (rank 44) & 1 & -3.33 & 0.31 & 0.02 & Other synovitis and tenosynovitis (727.09) \\
\hline Rs Cp (rank 46) & 1 & -3.40 & 0.30 & 0.03 & Sarcoidosis (135) \\
\hline Rtu Cp (rank 47) & 1 & -3.48 & 0.30 & 0.03 & Tobacco use disorder (305.1) \\
\hline Rdf Cp (rank 48) & 1 & -4.05 & 0.26 & 0.04 & Chronic diastolic heart failure (428.32) \\
\hline MODEL & $\Delta \mathrm{df}$ & $\Delta \mathrm{BIC}$ & $\% \Delta \mathrm{H}$ & Alpha & Variable description \\
\hline \multicolumn{6}{|l|}{$\begin{array}{l}\text { COARSE, best model } \\
\text { (loopless) }\end{array}$} \\
\hline \multicolumn{6}{|l|}{$\Delta$ BIC (best model) } \\
\hline Ruh Rrd Cp & 3 & 58.21 & 5.04 & 0.00 & $\begin{array}{l}\text { Other and unspecified hyperlipidemia (272.4), } \\
\text { Unspecified hypertensive renal disease (403.9) }\end{array}$ \\
\hline \multicolumn{6}{|l|}{$\triangle \mathrm{AIC}$ (best model) } \\
\hline Ruh Ros Raf Rku Cp & 15.0 & 4.62 & 7.89 & 0.00 & $\begin{array}{l}\text { Other and unspecified hyperlipidemia (272.4) + } \\
\text { Obstructive sleep apnea (327.23) + Atrial } \\
\text { fibrillation (427.31) + Chronic kidney disease, } \\
\text { unspecified (585.9) }\end{array}$ \\
\hline \multicolumn{6}{|l|}{ Inc.P (best model) } \\
\hline Ruh Ros Raf Rku Cp & 15.0 & 4.62 & 7.89 & 0.00 & $\begin{array}{l}\text { Other and unspecified hyperlipidemia (272.4) + } \\
\text { Obstructive sleep apnea (327.23) + Atrial } \\
\text { fibrillation (427.31) + Chronic kidney disease, } \\
\text { unspecified (585.9) }\end{array}$ \\
\hline MODEL & $\Delta \mathrm{df}$ & $\triangle \mathrm{BIC}$ & $\% \Delta \mathrm{H}$ & Alpha & Variable description \\
\hline \multicolumn{6}{|l|}{$\begin{array}{l}\text { FINE, best models } \\
\text { (with loops) }\end{array}$} \\
\hline \multicolumn{6}{|l|}{$\Delta \mathrm{BIC}$ (best model) } \\
\hline $\begin{array}{l}\text { Ruh Raf Cp : Ros Cp : Rhd } \\
\text { Cp : Rav Cp : Reg Cp : Rku } \\
\text { Cp : Ruu Cp : Rro Cp }\end{array}$ & 10 & 87.76 & 10.39 & 0.00 & $\begin{array}{l}\text { Other and unspecified hyperlipidemia (272.4) + } \\
\text { Atrial fibrillation (427.31), Obstructive sleep } \\
\text { apnea (327.23), Other chronic pulmonary heart } \\
\text { disease (416.8), Aortic valve disorders (424.1), } \\
\text { Esophagitis (530.1), Chronic kidney disease, } \\
\text { unspecified (585.9), Other disorders of urethra } \\
\text { and urinary tract (599), Rosacea (695.3) }\end{array}$ \\
\hline
\end{tabular}




\begin{tabular}{|c|c|c|c|c|c|}
\hline MODEL & $\Delta \mathrm{df}$ & $\Delta \mathrm{BIC}$ & $\% \Delta \mathrm{H}$ & Alpha & Variable description \\
\hline \multicolumn{6}{|c|}{ Inc.P \& $\Delta \mathrm{AIC}$ (same best model) } \\
\hline $\begin{array}{l}\text { Rs Cp : Ruh Raf Cp : Rmo } \\
\text { Cp : Rtu Raf Cp : Ros Rhd } \\
\text { Cp : Rpp Raf Cp : Rlb Cp : } \\
\text { Rhe Rro Cp : Rhe Rsy Cp : } \\
\text { Rca Cp : Rav Cp : Rdf Cp : } \\
\text { Rcb Cp : Reg Cp : Rkd Cp : } \\
\text { Rku Cp : Ruu Cp : Rpy Cp }\end{array}$ & 27 & 87.02 & 16.57 & 0.00 & $\begin{array}{l}\text { Sarcoidosis (135), Other and unspecified } \\
\text { hyperlipidemia (272.4) + Atrial fibrillation } \\
\text { (427.31), Morbid Obesity (278.01), Tobacco use } \\
\text { disorder (305.1) + Atrial fibrillation (427.31), } \\
\text { Obstructive sleep apnea (327.23) + Other } \\
\text { chronic pulmonary heart disease (416.8), } \\
\text { Unspecified hereditary and idiopathic peripheral } \\
\text { neuropathy (356.9) + Atrial fibrillation (427.31), } \\
\text { Legal blindness (369.4), Unspecified essential } \\
\text { hypertension (401.9) + Rosacea (695.3), } \\
\text { Unspecified essential hypertension (401.9) + } \\
\text { Other synovitis and tenosynovitis (727.09), } \\
\text { Coronary atherosclerosis of native coronary } \\
\text { artery (414.01), Aortic valve disorders (424.1), } \\
\text { Chronic diastolic heart failure (428.32), } \\
\text { Obstructive chronic bronchitis (491.2), } \\
\text { Esophagitis (530.1), Chronic kidney disease, } \\
\text { Stage III (585.3), Chronic kidney disease } \\
\text { (585.9), Other disorders of urethra and urinary } \\
\text { tract (599), Polymyalgia rheumatica (725) }\end{array}$ \\
\hline
\end{tabular}

- Fine Searches (Models with Loops)

Performing a search that allowed for loops with only Comorbidity IVs (Table 22) resulted in the best BIC model Ruh Raf Cp : Ros Cp : Rhd Cp : Rav Cp : Reg Cp : Rku Cp : Ruu Cp : Rro Cp, with a corresponding reduction in uncertainty of $10.39 \%$, a very slight improvement over the model in Table 20, namely Ageb Cp : Nrb Cp : Ruh Cp : Rhd Cp : Rky $\mathbf{C p}$ : Rro $\mathbf{C p}$, at a cost of an increase of complexity: $\Delta \mathrm{df}=10$, compared to 8. The first component of this model, Ruh Raf $\mathbf{C p}$ shows an interaction effect between Ruh and Raf in their combined effect on the DV. Thus, in the best model found that using Comorbidities IVs, other and unspecified hyperlipidemia (Ruh) and atrial fibrillation (Raf) form one predictive component, followed by obstructive sleep apnea (Ros), other chronic pulmonary heart disease (Rhd), aortic valve disorders (Rav), esophagitis (Reg), 
chronic kidney disease, unspecified (Rku), other disorders of urethra and urinary tract (Ruu), and rosacea (Rro).

Similar to the results from Table 20, the best model from Table 21, Ruh Raf Cp : Ros Cp : Rhd Cp : Rav Cp : Reg Cp : Rku Cp : Ruu Cp : Rro Cp, also excludes Rrd (the top single predicting variable in the Comorbidity only search). Just like the model in Table 20, the other variables in the model presumably cover the information in Rrd.

\section{- Comparing Search Results (Knee, Cp)}

The All IVs and the Comorbidity IVs searches yield two sets of results that can be compared to each other and interpreted with a 3-tiered classification of results, as described below and summarized in Table 23.

Table 23. The 3-Tiered Classification of Predicting Variables for DV: Complication, Knee Analysis

\begin{tabular}{|l|l|}
\hline Tier & Variables \\
\hline Tier 1 - Most Important & Nrb, Ageb, Ruh, Rhd, Rku \\
\hline Tier 2 & Reg, Raf, Ros, Rav, Ruu \\
\hline Tier 3 & Rro \\
\hline
\end{tabular}

Tier 1 contains variables from the best-by-BIC model from the dataset All IVs. This is the most complete search, and the one that provides the most conservative predictors. The selected variables (Nrb, Ageb, Ruh, Rhd, Rku, Rro) are shown in the first row of Table 23, and are considered the most important predicting variables.

Tier 2 contains variables not in Tier1, but found in the AIC (less conservative) model of All IVs AND in the BIC model of Comorbidity IVs. For the Knee Analysis of DV Complication, this selects the variables Reg, Raf, Ros, Rav, Ruu as the next-most important predicting variables. These are shown in the second row of Table 23. 
Finally, this classification places into Tier 3 any variables present elsewhere in the best model search results, but not included in Tier 1 or Tier 2 . That is, any variables unique to one of the two searches: variables in the AIC model of All IVs but not in the BIC model of Comorbidity IVs, as well as variables in the BIC model of Comorbidity IVs but not in the AIC model from All IVs. In this case, there were no variables that met these specifications. Strictly speaking, by the criteria set forth for Tier 1, Rro should have been included, but it has been "demoted" to Tier3 for reasons that will be explained below.

\section{- Model FIT}

Having found a best model, the next step is to analyze its detailed content; i.e., the conditional probability distribution for the DV, given the predicting IVs. This distribution is shown in Table 24 below, for the best fine-grained model from the search with All IVs, namely Ageb Cp : Nrb Cp : Ruh Cp : Rhd Cp : Rku Cp : Rro Cp. The columns of the table are: the model number, to be able to refer to models easily; the six IVs in the model and their different states; the frequency of each particular IV (vector) state; the conditional probability $\mathrm{p}(\mathbf{C p}=0 \mid \mathrm{IV})$ and $\mathrm{p}(\mathbf{C p}=1 \mid \mathrm{IV})$ in the data given as percentages (so they add up to 100\%); these two conditional probabilities in the model (which is an approximation to the data) written as $\mathrm{q}(\mathbf{C p}=0 \mid \mathrm{IV})$ and $\mathrm{q}(\mathbf{C p}=1 \mid \mathrm{IV})$; the "risk ratio" of $\mathrm{q}(\mathbf{C p}=1 \mid \mathrm{IV}) / \mathrm{q}(\mathbf{C p}=1)$; i.e., the probability of complications for a particular IV state divided by the marginal probability of complications for the whole sample. So, for example, the first row specifies the IV state (Ageb, Nrb, Ruh, Rhd, Rku, Rro) = $(1,1,0,0,0,0)$, which occurs 502 times in the sample, for which the conditional probabilities for the data (p) and the model (q) are given in percent, where ratio 
$0.19=0.89 / 4.73$, and where the p-value for the comparison of $(99.11,0.89)$ to the

margins $(95.27,4.73)$ is 0 . The "risk ratio" conveys the effect size, while the p-value

conveys the significance of the effect size. The p-values are important and are used to

retain significant results only; however, it is the ratio - the effect size - that is used in

selecting the states that result in an effect, either protective (blue) or risky (orange), 10\%

above or below the risk ratio of 1 .

Table 24. Full Fit Table (Knee) All IVs for Best Model:

Ageb Cp : Nrb Cp : Ruh Cp : Rhd Cp : Rku Cp : Rro Cp.

Blue rows indicate ratio $<0.90$ and orange rows indicate ratio $>1.10$.

(Ratios between 0.91 and 1.09 are close to the margins and are indicated in gray.)

\begin{tabular}{|c|c|c|c|c|c|c|c|c|c|c|c|c|c|}
\hline \multicolumn{7}{|c|}{ IVs } & \multicolumn{3}{|l|}{ Data } & \multirow{2}{*}{\multicolumn{2}{|c|}{$\begin{array}{l}\text { Model } \\
\text { calc. } \mathrm{q}(\mathrm{DV} \mid \mathrm{IV})\end{array}$}} & \multirow[b]{3}{*}{ ratio } & \multirow[b]{3}{*}{$\mathrm{p}($ margin $)$} \\
\hline & & & & & & & & obs. p & V|IV) & & & & \\
\hline$\#$ & Ageb & $\mathrm{Nrb}$ & Ruh & Rhd & Rku & Rro & freq & $\mathrm{Cp}=0$ & $\mathrm{Cp}=1$ & $\mathrm{Cp}=0$ & $\mathrm{Cp}=1$ & & \\
\hline 1 & 1 & 1 & 0 & 0 & 0 & 0 & 502 & 99.00 & 1.00 & 99.11 & 0.89 & 0.19 & 0.00 \\
\hline 2 & 1 & 1 & 1 & 0 & 0 & 0 & 1 & 100.00 & 0.00 & 98.46 & 1.54 & 0.33 & 0.88 \\
\hline 3 & 1 & 2 & 0 & 0 & 0 & 0 & 457 & 98.69 & 1.31 & 97.77 & 2.24 & 0.47 & 0.01 \\
\hline 4 & 1 & 2 & 0 & 0 & 0 & 1 & 1 & 100.00 & 0.00 & 80.86 & 19.14 & 4.05 & 0.50 \\
\hline 5 & 1 & 2 & 0 & 0 & 1 & 0 & 2 & 100.00 & 0.00 & 91.86 & 8.14 & 1.72 & 0.82 \\
\hline 6 & 1 & 2 & 0 & 1 & 0 & 0 & 1 & 0.00 & 100.00 & 87.38 & 12.62 & 2.67 & 0.71 \\
\hline 7 & 1 & 2 & 1 & 0 & 0 & 0 & 34 & 91.18 & 8.82 & 96.17 & 3.83 & 0.81 & 0.81 \\
\hline 8 & 1 & 3 & 0 & 0 & 0 & 0 & 380 & 96.05 & 3.95 & 95.90 & 4.10 & 0.87 & 0.56 \\
\hline 9 & 1 & 3 & 0 & 0 & 0 & 1 & 1 & 100.00 & 0.00 & 69.34 & 30.66 & 6.48 & 0.22 \\
\hline 10 & 1 & 3 & 0 & 0 & 1 & 0 & 8 & 100.00 & 0.00 & 85.80 & 14.20 & 3.00 & 0.24 \\
\hline 11 & 1 & 3 & 0 & 1 & 0 & 0 & 2 & 100.00 & 0.00 & 78.75 & 21.25 & 4.49 & 0.27 \\
\hline 12 & 1 & 3 & 1 & 0 & 0 & 0 & 96 & 89.58 & 10.42 & 93.07 & 6.93 & 1.47 & 0.31 \\
\hline 13 & 1 & 3 & 1 & 0 & 0 & 1 & 1 & 100.00 & 0.00 & 56.47 & 43.53 & 9.21 & 0.07 \\
\hline 14 & 1 & 3 & 1 & 0 & 1 & 0 & 3 & 66.67 & 33.33 & 77.61 & 22.39 & 4.74 & 0.15 \\
\hline 15 & 1 & 3 & 1 & 1 & 0 & 0 & 1 & 0.00 & 100.00 & 68.01 & 31.99 & 6.77 & 0.20 \\
\hline 16 & 2 & 1 & 0 & 0 & 0 & 0 & 421 & 99.29 & 0.71 & 98.78 & 1.22 & 0.26 & 0.00 \\
\hline 17 & 2 & 1 & 0 & 1 & 0 & 0 & 1 & 100.00 & 0.00 & 92.78 & 7.22 & 1.53 & 0.91 \\
\hline 18 & 2 & 1 & 1 & 0 & 0 & 0 & 6 & 100.00 & 0.00 & 97.90 & 2.10 & 0.44 & 0.76 \\
\hline 19 & 2 & 2 & 0 & 0 & 0 & 0 & 420 & 96.91 & 3.10 & 96.96 & 3.04 & 0.64 & 0.10 \\
\hline 20 & 2 & 2 & 1 & 0 & 0 & 0 & 50 & 90.00 & 10.00 & 94.82 & 5.18 & 1.10 & 0.88 \\
\hline 21 & 2 & 3 & 0 & 0 & 0 & 0 & 349 & 93.98 & 6.02 & 94.47 & 5.53 & 1.17 & 0.48 \\
\hline 22 & 2 & 3 & 0 & 0 & 0 & 1 & 3 & 33.33 & 66.67 & 62.26 & 37.74 & 7.98 & 0.01 \\
\hline 23 & 2 & 3 & 0 & 0 & 1 & 0 & 10 & 60.00 & 40.00 & 81.51 & 18.49 & 3.91 & 0.04 \\
\hline 24 & 2 & 3 & 0 & 1 & 0 & 0 & 3 & 66.67 & 33.33 & 73.00 & 27.00 & 5.71 & 0.07 \\
\hline 25 & 2 & 3 & 0 & 1 & 1 & 0 & 1 & 100.00 & 0.00 & 41.10 & 58.90 & 12.46 & 0.01 \\
\hline 26 & 2 & 3 & 1 & 0 & 0 & 0 & 137 & 95.62 & 4.38 & 90.74 & 9.26 & 1.96 & 0.01 \\
\hline 27 & 2 & 3 & 1 & 0 & 1 & 0 & 9 & 44.44 & 55.56 & 71.66 & 28.34 & 5.99 & 0.00 \\
\hline 28 & 2 & 3 & 1 & 1 & 0 & 0 & 1 & 100.00 & 0.00 & 60.80 & 39.21 & 8.29 & 0.11 \\
\hline 29 & 3 & 1 & 0 & 0 & 0 & 0 & 376 & 97.87 & 2.13 & 98.11 & 1.90 & 0.40 & 0.01 \\
\hline
\end{tabular}




\begin{tabular}{|c|c|c|c|c|c|c|c|c|c|c|c|c|c|}
\hline 30 & 3 & 1 & 1 & 0 & 0 & 0 & 2 & 50.00 & 50.00 & 96.74 & 3.26 & 0.69 & 0.92 \\
\hline 31 & 3 & 2 & 0 & 0 & 0 & 0 & 447 & 95.08 & 4.92 & 95.32 & 4.68 & 0.99 & 0.96 \\
\hline 32 & 3 & 2 & 0 & 0 & 0 & 1 & 1 & 0.00 & 100.00 & 66.30 & 33.71 & 7.13 & 0.17 \\
\hline 33 & 3 & 2 & 0 & 0 & 1 & 0 & 7 & 100.00 & 0.00 & 84.01 & 15.99 & 3.38 & 0.19 \\
\hline 34 & 3 & 2 & 1 & 0 & 0 & 0 & 54 & 94.44 & 5.56 & 92.11 & 7.89 & 1.67 & 0.27 \\
\hline 35 & 3 & 3 & 0 & 0 & 0 & 0 & 341 & 90.62 & 9.38 & 91.60 & 8.41 & 1.78 & 0.00 \\
\hline 36 & 3 & 3 & 0 & 0 & 0 & 1 & 2 & 100.00 & 0.00 & 51.29 & 48.71 & 10.30 & 0.00 \\
\hline 37 & 3 & 3 & 0 & 0 & 1 & 0 & 28 & 75.00 & 25.00 & 73.77 & 26.23 & 5.55 & 0.00 \\
\hline 38 & 3 & 3 & 0 & 1 & 0 & 0 & 7 & 57.14 & 42.86 & 63.31 & 36.70 & 7.76 & 0.00 \\
\hline 39 & 3 & 3 & 0 & 1 & 1 & 0 & 1 & 100.00 & 0.00 & 30.81 & 69.19 & 14.63 & 0.00 \\
\hline 40 & 3 & 3 & 1 & 0 & 0 & 0 & 148 & 87.84 & 12.16 & 86.21 & 13.79 & 2.92 & 0.00 \\
\hline 41 & 3 & 3 & 1 & 0 & 0 & 1 & 1 & 0.00 & 100.00 & 37.65 & 62.35 & 13.19 & 0.01 \\
\hline 42 & 3 & 3 & 1 & 0 & 1 & 0 & 18 & 66.67 & 33.33 & 61.74 & 38.26 & 8.09 & 0.00 \\
\hline 43 & 3 & 3 & 1 & 1 & 0 & 0 & 2 & 50.00 & 50.00 & 49.74 & 50.26 & 10.63 & 0.00 \\
\hline & & & & & & & 4336 & 95.27 & 4.73 & 95.27 & 4.73 & 1.00 & \\
\hline$\#$ & Ageb & Nrb & Ruh & Rhd & Rku & Rro & freq & $\mathrm{Cp}=0$ & $\mathrm{Cp}=1$ & $\mathrm{Cp}=0$ & $\mathrm{Cp}=1$ & ratio & $\mathrm{p}$ (margin) \\
\hline
\end{tabular}

The values for the All IVs: number of risks binned (Nrb) and age binned (Ageb)

are either states 1,2, or 3 and are the three bins that the data was discretized to. Bin ranges and frequencies are summarized in Table 25 and Table 26 below. With three states possible for each of Ageb and Nrb, and two states possible for each of Ruh, Rhd, Rku and Rro, one might expect to see 144 rows — one row for each possible combination of states. However, fit tables don't show rows for IV states that did not occur in the data. In Table 24, for example, instead of 144 rows there are 43 rows. Additionally, fit tables in the remainder of the results section will include only the rows that meet the protocol of this project, which requires a frequency of 10 or more and a p(margin) of 0.05 or less.

Table 25. Number of Risks (Nrb) Bin Range \& Frequency (Knee)

\begin{tabular}{|c|c|c|}
\hline Bin & Range & Frequency \\
\hline 1 & $0-1$ & 1309 \\
\hline 2 & $2-3$ & 1474 \\
\hline 3 & $4-18$ & 1553 \\
\hline
\end{tabular}


Table 26. Age Binned (Ageb) Bin Range

\& Frequency (Knee).

\begin{tabular}{|c|c|c|}
\hline Bin & Range & Frequency \\
\hline 1 & $32-62$ & 1,490 \\
\hline 2 & $63-71$ & 1,411 \\
\hline 3 & $72-95$ & 1,435 \\
\hline
\end{tabular}

For the independence model, which is the reference, we do not know the state of Ageb or Nrb or if a comorbidity was present, so all of the uncertainty of the DV comes from its marginal distribution, which is the last line of the table, for which the data and model conditional probabilities are the same. For the calculated model, knowing the bin states of All IVs (Nrb, Ageb) or the presence or absence of the individual Comorbidity IVs (Ruh, Rhd, Rku, Rro) tells us about the probability of $\mathbf{C p}$ occurring. The model conditional probabilities are more appropriate to use than the observed (data) conditional probabilities because the model is simpler than the data and thus generalizes better.

Each of the model's components, namely Ageb $\mathbf{C p}$ or Nrb $\mathbf{C p}$ or Ruh $\mathbf{C p}$ or Rhd Cp or Rku Cp or Rro $\mathbf{C p}$, has an individual conditional probability distribution and is individually informative. Table 27 is a compressed table summarizing the conditional probability distributions for one component only. For example, looking only at Age (Table 27), the conditional probability of $\mathbf{C p}=1$ given Ageb $=1$ is $2.82 \%$ with the risk ratio of 0.60 (row 9), Ageb $=2$ has a neutral risk ratio close to 1 and was excluded, and Ageb $=3$ shows increased risk with a ratio $=1.52$ (row 6$)$. In other words, knowing only Ageb, regardless of the states of the other IVs in the model, there is a significant difference in the probability of $\mathbf{C p}=\mathbf{1}$; i.e., a significant difference in the probability of complications between the Ageb values - a decrease in risk for the low bin and an increase in risk for the high bin. 
The individual model component's projections alone do not explain the DV as fully as the joint probability distribution (the calculated distribution of the entire model). Table 27 shows that Ageb high (bin = 3) predicts an increase in risk; yet in the table for the model that combines these components (Table 24), Ageb $=3$ appears in row 29 along with $\mathrm{Nrb}=1$ and Ruh, Rhd, Rku and Rro all absent that has a significantly reduced risk ratio of 0.40. This is supported by Table 28, which looks at the Nrb Cp component alone, which suggests that when $\mathrm{Nrb}=1$, the risk is reduced (ratio $=0.27$ ) as compared to when $\mathrm{Nrb}=3$ and the risk is increased (ratio $=1.82$ ). On the other hand, when Ruh, Rhd, Rku and Rro are present, the risk increases with a sizeable effect, as seen in the condensed component table (Table 29) for the model's comorbidity components (ratio $=2.29,7.40$, 5.59 , and 8.46 , respectively).

Table 27. Component Fit Table for IVAgeb in (Knee) All IVs Best Model: Ageb Cp : Nrb Cp : Ruh Cp : Rhd Cp : Rku Cp : Rro Cp.

Blue rows indicate ratio $<0.90$ and orange rows indicate ratio $>1.10$. (Ratios between 0.91 and 1.09 are close to the margins and are excluded.)

\begin{tabular}{|c|c|c|c|c|c|c|}
\hline & IV & Data & \multicolumn{2}{|c|}{ obs. $\mathrm{p}$ (DV|IV) } & & \\
\hline$\#$ & Ageb & freq & $\mathrm{Cp}=0$ & $\mathrm{Cp}=1$ & Ratio & $\mathrm{p}$ (margin) \\
\hline 1 & 1 & 1490 & 97.18 & 2.82 & 0.60 & 0.00 \\
\hline 3 & 3 & 1435 & 92.82 & 7.18 & 1.52 & 0.00 \\
\hline & & 4336 & 95.27 & 4.73 & 1.00 & \\
\hline
\end{tabular}

Table 28. Component Fit Table for IV Nrb in (Knee) All IVs Best Model: Ageb Cp : Nrb Cp : Ruh Cp : Rhd Cp : Rku Cp : Rro Cp.

Blue rows indicate ratio $<0.90$ and orange rows indicate ratio $>1.10$. (Ratios between 0.91 and 1.09 are close to the margins and are excluded.)

\begin{tabular}{|c|c|c|c|c|c|c|}
\hline & IV & Data & \multicolumn{2}{|c|}{ obs. $\mathrm{p}(\mathrm{DV} \mid \mathrm{IV})$} & & \\
\hline$\#$ & Nrb & freq & $\mathrm{Cp}=0$ & $\mathrm{Cp}=1$ & Ratio & $\mathrm{p}$ (margin) \\
\hline 1 & 1 & 1309 & 98.70 & 1.30 & 0.27 & 0.00 \\
\hline 2 & 2 & 1474 & 96.34 & 3.66 & 0.77 & 0.05 \\
\hline 3 & 3 & 1553 & 91.37 & 8.63 & 1.82 & 0.00 \\
\hline & & 4336 & 95.27 & 4.73 & 1.00 & \\
\hline
\end{tabular}


Table 29. Condensed Fit Table of Comorbidity Components for

IVs Ruh, Rhd, Rku, Rro in (Knee) All IVs Best Model:

Ageb Cp : Nrb Cp : Ruh Cp : Rhd Cp : Rku Cp : Rro Cp.

Blue rows indicate ratio $<0.90$ and orange rows indicate ratio $>1.10$.

(Ratios between 0.91 and 1.09 are close to the margins and are excluded.)

\begin{tabular}{|c|c|c|c|c|c|c|}
\hline & & Data & \multicolumn{2}{|c|}{ obs. $\mathrm{p}$ (DV|IV) } & & \\
\hline IV & State & freq & $\mathrm{Cp}=0$ & $\mathrm{Cp}=1$ & Ratio & $\mathrm{p}$ (margin) \\
\hline Ruh & 0 & 3772 & 96.18 & 3.82 & 0.81 & 0.01 \\
\hline Ruh & 1 & 564 & 89.18 & 10.82 & 2.29 & 0.00 \\
\hline Rhd & 1 & 20 & 65.00 & 35.00 & 7.40 & 0.00 \\
\hline Rku & 1 & 87 & 73.56 & 26.44 & 5.59 & 0.00 \\
\hline Rro & 1 & 10 & 60.00 & 40.00 & 8.46 & 0.00 \\
\hline & & 4336 & 95.27 & 4.73 & 1.00 & \\
\hline
\end{tabular}

The marginal distribution (last line) of the integrated Table 24 above shows that in the total population of 4,336 knee replacement cases, Complication (Cp) was actually present (observed) in $4.73 \%$ and was absent in $95.27 \%$ of the cases. If the conditional probabilities for particular IV states are higher or lower than the margins, then the IVs have provided new (predictive) information. Looking at the conditional probabilities of the model in Table 24 shows a number of rows whose calculated probabilities appear very different from the margins (the blue and orange shaded cells).

For Table 24, rows are highlighted with $\mathrm{p}$ (margin) $\leq 0.05$ and frequency $>10$. The distribution for $\mathbf{C p}$ is highly skewed, since $\mathbf{C p}=1$ occurs in only $4.73 \%$ of the cases; therefore, only if a model predicts more than $50 \%$ chance of $\mathbf{C p}=1$ will the prediction rule be to predict "yes" for Cp. This occurs only for IV states where the frequencies are 1 or 2 (rows 25,39 , and 41), and predictions with such low frequencies are here judged not significant even if their calculated p-values are under the typical 0.05 threshold (because of their extreme difference from the margins). In fact, in the tiered search results (Table 23) Rro had been demoted from Tier 1 to Tier 3 precisely for this reason. Rro did not appear in any of the IV states after the removal of IV states with a frequency $<10$ 
even though the p-value had been significant. Aside from these very low-frequency IV states, the model distribution always predicts $\mathbf{C p}=0$, which is just what the marginal distribution predicts even without any IV information. What the model predicts beyond the independence model is the risk of occurrence. While there were no IV states with sizeable frequencies that predicted greater than $50 \%$ probability of $\mathbf{C p}=$, there are probabilities that are significantly different than the margins, which demonstrate either a protective effect $(<4.73 \%)$ or a higher risk of $\mathbf{C p}=1(>4.73 \%)$. These effects are indicated in the column labeled "ratio" which is model q(DV|IV) for $\mathbf{C p}=1$ divided by its marginal $q(D V)$ value. It is the probability of a complication for a type of patient divided by probability of complication for the full sample. When this ratio is $<0.90$ (and is statistically significant), risk is reduced (blue cells); when the ratio is $>1.10$ (and statistically significant), risk is increased (orange cells).

Row 1 (Table 24), for example, shows a protective effect of age binned $($ Ageb) $<63$ (bin $=1)$ and number of risks binned $(\mathrm{Nrb})$ is low $(\mathrm{bin}=1)$ with number of risks $\leq 1$ with the probability of $\mathbf{C p}=1$ at $0.89 \%$ (ratio $=0.19$ ), markedly lower than the margin of $4.73 \%$. Row 16 shows a similar protective effect, where even with Ageb range 63-71 (bin 2), as long as Nrb < 1 (bin 1), the probability is $1.22 \%$, which is lower than the margin (ratio $=0.26$ ). Row 29 (Table 24$)$ also offers a protective effect where even with Ageb range 72-95 (bin3) as long as $\mathrm{Nrb}<1$ (bin 1), then the probability of $\mathbf{C p}=1$ is still lower than the margin at $2.13 \%$ (ratio $=0.40$ ). Row 3 (Table 24) shows that even where there is an increase in number of comorbidities with $\mathrm{Nrb}=2$ or 3 (bin 2), when Ageb $=1$, there is still a protective effect with probability of $\mathbf{C p}=1$ of $2.24 \%$ $($ ratio $=0.47)$. 
In each of these three cases where there was a protective effect, the four Comorbidity IVs, Ruh, Rhd, Rku and Rro, were all absent. To recapitulate: the results show that if these Comorbidity IVs are absent and $\mathrm{Nrb}=1$, then Ageb can be in any of its three potential states and the risk is still low. Risk is also reduced if Ruh, Rhd, Rku and Rro are not present, even if there are more comorbidities present $(\mathrm{Nrb}=2)$, if the age is low $($ Ageb = 1).

Row 35 (Table 24) shows IV states that predict higher risk of $\mathbf{C p}=1$. With Ageb 72-95 (bin 3), and Nrb between 4 and 18 (bin 3), there is a higher probability of $\mathbf{C p}$ at $8.41 \%($ ratio $=1.78)$. In this state, there was no presence of one of the four Comorbidity IVs (Ruh, Rhd, Rku \& Rro). In row 23, however, with the presence of Rku and with lower Ageb 63-71 (bin 2), and with Nrb again between 4 and 18 (bin 3), the risk of Cp is $18.49 \%($ ratio $=3.91)$.

Compare row 35 also with row 37 in Table 24 (freq $=28)$, where again Ageb $=3$ and $\mathrm{Nrb}=3$, but $\mathrm{Rku}$ is present and we get a much higher risk ratio of 5.5, a 0.2623 probability of $\mathbf{C p}=1$ which is over five times the risk of the whole sample.

One way of summarizing the model predictions is through the use of a decision tree. The decision tree provides an operational branching of questions one could ask, and the answers that result. Before looking at the decision tree for model Ageb $\mathbf{C p}: \mathrm{Nrb} \mathbf{C p}$ : Ruh Cp : Rhd Cp : Rku Cp : Rro Cp, three decision trees are shown for a much simpler model in order to explain the meaning of the decision tree and show how these decision trees will be constructed for the remainder of the analyses. In this example, the best BIC model from the All IVs loopless model search (Table 20) is used: Nrb Rku Cp. The fit table for this model is shown in Table 30 below. 
Table 30. Fit Table (Knee) All IVs for Best Coarse Model: Nrb Rku Cp.

Blue rows indicate ratio $<0.90$ and orange rows indicate ratio $>1.10$.

(IV states with frequency $<\mathbf{1 0}$ are not highlighted.)

\begin{tabular}{|c|c|c|c|c|c|c|c|c|c|}
\hline & \multicolumn{2}{|c|}{ IVs } & \multicolumn{3}{c|}{ Data } & \multicolumn{2}{c|}{ Model } & \multicolumn{2}{c|}{} \\
\hline & \multicolumn{2}{|c|}{} & & \multicolumn{2}{c|}{ obs. $\mathrm{p}(\mathrm{DV} \mid \mathrm{IV})$} & \multicolumn{2}{c|}{ calc. $\mathrm{q}(\mathrm{DV} \mid \mathrm{IV})$} & & \\
\hline$\#$ & Nrb & Rku & freq & $\mathrm{Cp}=0$ & $\mathrm{Cp}=1$ & $\mathrm{Cp}=0$ & $\mathrm{Cp}=1$ & ratio & $\mathrm{p}$ (margin) \\
\hline 1 & 1 & 0 & 1309 & 98.70 & 1.30 & 98.70 & 1.30 & 0.27 & 0.00 \\
\hline 2 & 2 & 0 & 1465 & 96.31 & 3.69 & 96.31 & 3.69 & 0.78 & 0.06 \\
\hline 3 & 2 & 1 & 9 & 100.00 & 0.00 & 100.00 & 0.00 & 0.00 & 0.53 \\
\hline 4 & 3 & 0 & 1475 & 92.48 & 7.53 & 92.48 & 7.53 & 1.59 & 0.00 \\
\hline 5 & 3 & 1 & 78 & 70.51 & 29.49 & 70.51 & 29.49 & 6.24 & 0.00 \\
\hline & & & 4336 & 95.27 & 4.73 & 95.27 & 4.73 & 1.00 & \\
\hline
\end{tabular}

These results from the fit table can be communicated in a decision tree. The full decision tree, showing all possible combinations of IV states, is not included in this project as the relevance and size are prohibitive. The decision trees used in this project omit IV states that are not observed $(\mathrm{Nrb}=1, \mathrm{Rku}=1)$ or whose distribution is not significantly different from the marginal distribution. The protocol in this project selects only scenarios with a frequency $\geq 10$ and a $\mathrm{p}($ margin $) \leq 0.05$, and these rows were excluded in the fit tables as well as the corresponding branching on the decision trees. The simplified version of the decision tree from Table 30 is illustrated below in Figure 6 .

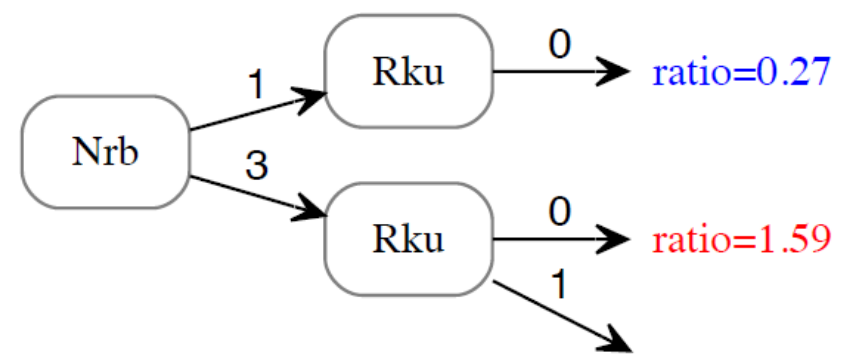

ratio $=6.24$

Figure 5. Simplified Decision Tree for DV Any Comp (Knee) All IVs, for Best Coarse Model Nrb Rku Cp. 
The decision tree for model Ageb Cp : Nrb Cp : Ruh Cp : Rhd Cp : Rku Cp :

Rro Cp (Table 24) is shown in Figure 6. Note that while Rro appears in the BIC model, none of the IV states in this table involving Rro $=1$ meets the criterion of $p$-value $<0.05$ and freq $\geq 10$. For this reason, Rro does not appear in the decision tree (Figure 6).

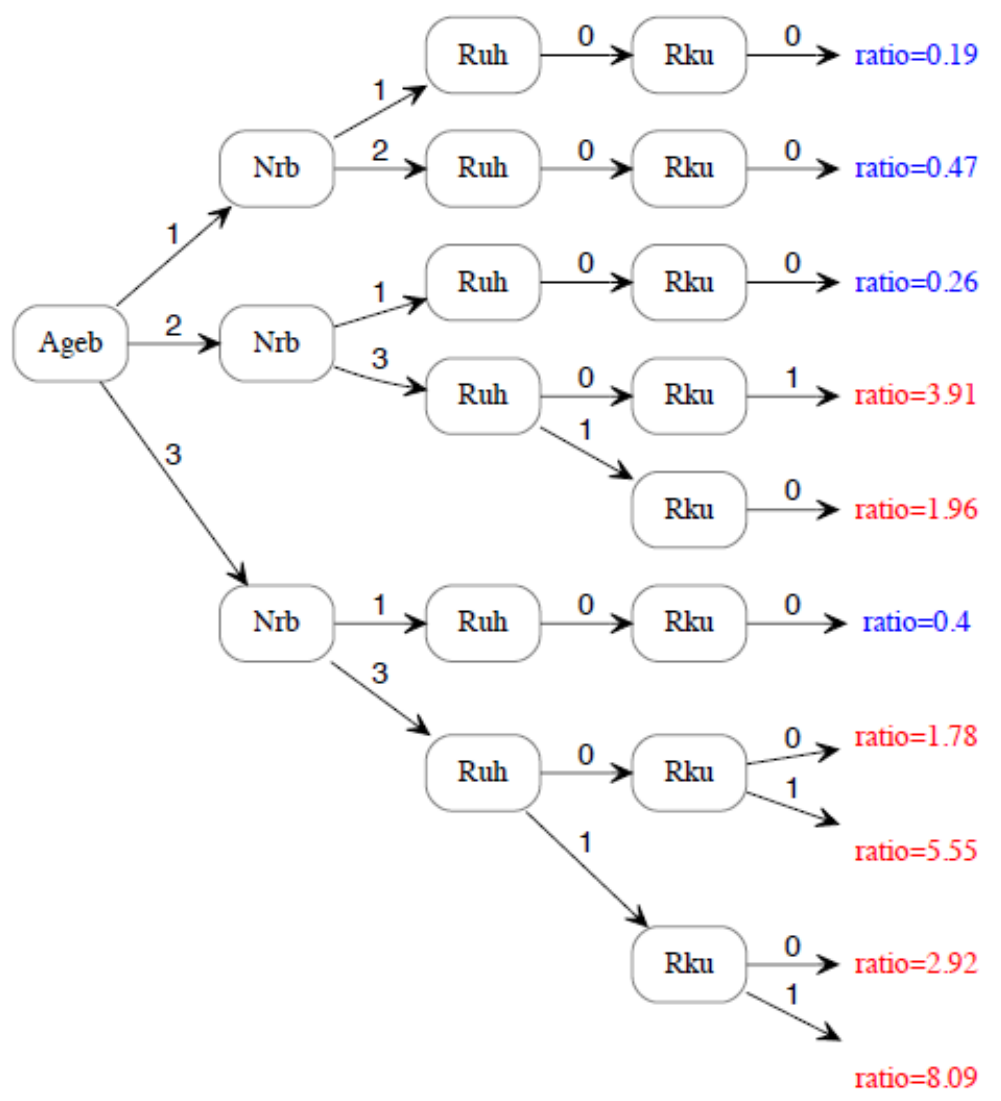

Figure 6. Decision Tree for DV Any Comp (Knee) All IVs for Best Model Ageb Cp : Nrb Cp : Ruh Cp : Rhd Cp : Rku Cp : Rro Cp.

The decision tree in Figure 6 shows clearly that regardless of Age (Ageb = 1, 2, or 3), as long as number of risks are low $(\mathrm{Nrb}=1)$ then the risk of Complication $\mathbf{C p}$ is substantially lower than 1 . If a patient has two or three comorbidities $(\mathrm{Nrb}=2)$, there is 
still lower risk as long as the patient is in the lowest age group (Ageb =1), which is 62 and younger.

The details of the best fine BIC model from the Comorbidity only search, Ruh Raf Cp : Ros Cp : Rhd Cp : Rav Cp : $\operatorname{Reg}$ Cp : Rku Cp : Ruu Cp : Rro Cp (Table 22), are given in Table 31. The Comorbidity IVs, Ruh, Ros, Rhd, Rav, Raf, Reg, Rku, Ruu, Rro, are listed in the nine IV columns. As discussed with Table 24, the probabilities of $\mathbf{C p}=1$ may differ significantly from the model's marginal probabilities. Each of the IVs is either 0 (comorbidity absent) or 1 (comorbidity present). In Table 31 , only $\mathrm{p}$ (margin) $<.05$ are shown due to the size of the table.

Row 1 (Table 31) is the case where all of the Comorbidity IVs are absent, with a frequency of 3,311 . Having none of these comorbidities lowers the risk ratio to 0.57 , where having any one of the comorbidities increases the risk of $\mathbf{C p}$. There are a few cases where having a particular comorbidity increases risk of $\mathbf{C p}=1$ substantially, such as is seen in rows $4($ ratio $=3.07), 6($ ratio $=1.9), 9($ ratio $=2.88), 24($ ratio $=7.32)$, and 29 $($ ratio $=3.57)$. 
Table 31. Fit Table (Knee) with Comorbidity IVs for Best Model Ruh Raf Cp : Ros Cp : Rhd Cp : Rav Cp : Reg Cp : Rku Cp : Ruu Cp : Rro Cp. Blue rows indicate ratio $<0.90$ and orange rows indicate ratio $>1.10$.

\begin{tabular}{|c|c|c|c|c|c|c|c|c|c|c|c|c|c|c|c|c|}
\hline \multicolumn{10}{|c|}{ IVs } & \multicolumn{3}{|l|}{ Data } & \multirow{2}{*}{\multicolumn{2}{|c|}{\begin{tabular}{|l} 
Model \\
calc. $q(\mathrm{DV} \mid \mathrm{IV})$ \\
\end{tabular}}} & \multirow[b]{3}{*}{ ratio } & \multirow[b]{3}{*}{$\mathrm{p}($ margin $)$} \\
\hline & & & & & & & & & & & \multicolumn{2}{|c|}{ obs. p(DV|IV) } & & & & \\
\hline \# & $\begin{array}{l}\mathrm{R} \\
\mathrm{u} \\
\mathrm{h}\end{array}$ & $\begin{array}{l}\mathrm{R} \\
\mathrm{o} \\
\mathrm{s}\end{array}$ & $\begin{array}{l}\mathrm{R} \\
\mathrm{h} \\
\mathrm{d}\end{array}$ & $\begin{array}{l}\mathrm{R} \\
\mathrm{a} \\
\mathrm{V}\end{array}$ & $\begin{array}{l}\mathrm{R} \\
\mathrm{a} \\
\mathrm{f}\end{array}$ & $\begin{array}{l}\mathrm{R} \\
\mathrm{e} \\
\mathrm{g}\end{array}$ & $\begin{array}{l}\mathrm{R} \\
\mathrm{k} \\
\mathrm{u}\end{array}$ & $\begin{array}{l}\mathrm{R} \\
\mathrm{u} \\
\mathrm{u}\end{array}$ & $\begin{array}{l}\mathrm{R} \\
\mathrm{r} \\
\mathrm{O}\end{array}$ & freq & $\mathrm{Cp}=0$ & $\mathrm{Cp}=1$ & $\mathrm{Cp}=0$ & $\mathrm{Cp}=1$ & & \\
\hline 1 & 0 & 0 & 0 & 0 & 0 & 0 & 0 & 0 & 0 & 3311 & 97.37 & 2.63 & 97.30 & 2.70 & 0.57 & 0.00 \\
\hline 2 & 0 & 0 & 0 & 0 & 0 & 0 & 1 & 0 & 0 & 45 & 82.22 & 17.78 & 85.46 & 14.54 & 3.07 & 0.00 \\
\hline 3 & 0 & 0 & 0 & 0 & 1 & 0 & 0 & 0 & 0 & 184 & 92.39 & 7.61 & 91.03 & 8.97 & 1.90 & 0.01 \\
\hline 4 & 1 & 0 & 0 & 0 & 0 & 0 & 0 & 0 & 0 & 442 & 92.08 & 7.92 & 92.07 & 7.93 & 1.68 & 0.00 \\
\hline 5 & 1 & 0 & 0 & 0 & 0 & 0 & 1 & 0 & 0 & 25 & 64.00 & 36.00 & 65.41 & 34.59 & 7.32 & 0.00 \\
\hline 6 & 1 & 1 & 0 & 0 & 0 & 0 & 0 & 0 & 0 & 35 & 82.86 & 17.14 & 83.11 & 16.89 & 3.57 & 0.00 \\
\hline & & & & & & & & & & 4336 & 95.27 & 4.73 & 95.27 & 4.73 & 1.00 & \\
\hline
\end{tabular}

The decision tree for Table 31 (Figure 7) shows that when unspecified hyperlipidemia (Ruh) is present, regardless of the states of the other Comorbidity IVs in the model, there is an increased risk of $\mathbf{C p}$. In fact, the only protective effect offered against $\mathbf{C p}$ occurring is to have none of the Comorbidity IVs. Having only 1 on the Comorbidity IVs Ruh, Rku, Ros, or Raf results in an increased risk ratio. The presence of both Ruh and Rku leads to the highest risk ratio of 7.32. 


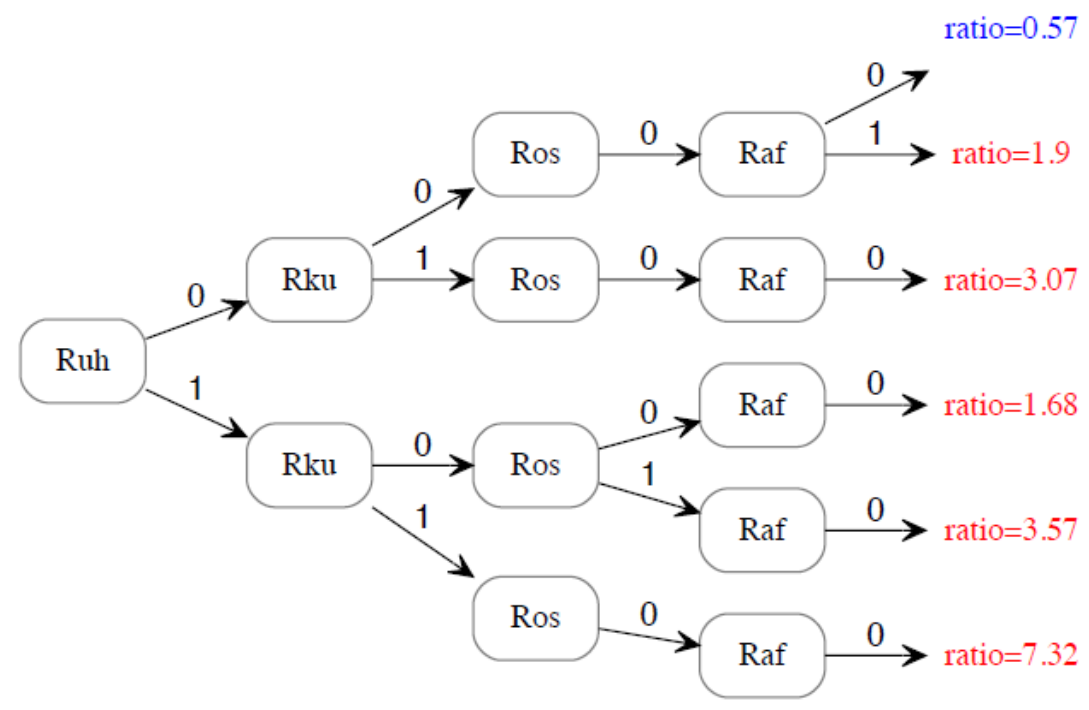

Figure 7. Decision Tree for DV Any Comp (Knee) with Comorbidity IVs for Best Model Ruh Raf Cp : Ros Cp : Rhd Cp : Rav Cp : Reg Cp : Rku Cp : Ruu Cp : Rro Cp.

Each of the model's components (Ruh, Ros, Rhd, Rav, Raf, Reg, Rku, Ruu, Rro), shown below, is individually informative in its conditional probability distribution. To illustrate with one of these components, Table 32 shows that when both Ruh and Raf (one component with two IVs, showing an interaction effect) are absent, there is a protective effect with a risk ratio of 0.71 . The risk ratio of $\mathbf{C p}$ increases when either Ruh or Raf is present (2.3 and 2.45, respectively), and when they are both present (2.2). It might seem odd that the ratio is lower when both are present than when only one is present, but the differences between these ratio values are probably not statistically significant. Table 33 shows the conditional probability distribution, with highest risk ratio at the top, of the other components in the model. 
Table 32. Component Fit Table for IVs Ruh Raf (Knee) Comorbidity IVs Best Model Ruh Raf Cp : Ros Cp : Rhd Cp : Rav Cp : Reg Cp : Rku Cp : Ruu Cp : Rro Cp. Blue rows indicate ratio $<0.90$ and orange rows indicate ratio $>1.10$.

\begin{tabular}{|c|c|c|c|c|c|c|c|}
\hline & \multicolumn{2}{|c|}{ IVs } & Data & \multicolumn{2}{c|}{ obs. $\mathrm{p}(\mathrm{DV} \mid \mathrm{IV})$} & & \\
\hline$\#$ & Ruh & Raf & freq & $\mathrm{Cp}=0$ & $\mathrm{Cp}=1$ & ratio & $\mathrm{p}$ (margin) \\
\hline 1 & 0 & 0 & 3565 & 96.63 & 3.37 & 0.71 & 0.00 \\
\hline 2 & 0 & 1 & 207 & 88.41 & 11.59 & 2.45 & 0.00 \\
\hline 3 & 1 & 0 & 516 & 89.15 & 10.85 & 2.30 & 0.00 \\
\hline 4 & 1 & 1 & 48 & 89.58 & 10.42 & 2.20 & 0.06 \\
\hline & & & 4336 & 95.27 & 4.73 & 1.00 & \\
\hline
\end{tabular}

Table 33. Condensed Component Fit Table for IVs Ruh Raf (Knee) Comorbidity IVs Best Model Ruh Raf Cp : Ros Cp : Rhd Cp : Rav Cp : Reg Cp : Rku Cp : Ruu Cp : Rro Cp. Orange rows indicate ratio $>1.10$.

(Ratios between 0.91 and 1.09 are close to the margins and are excluded.)

\begin{tabular}{|c|c|c|c|c|c|c|c|}
\hline & & & Data & \multicolumn{2}{|c|}{ obs. $\mathrm{p}(\mathrm{DV} \mid \mathrm{IV})$} & & \\
\hline$\#$ & IV & IV State & freq & $\mathrm{Cp}=0$ & $\mathrm{Cp}=1$ & ratio & $\mathrm{p}$ (margin) \\
\hline 1 & Reg & 1 & 6 & 50 & 50 & 10.58 & 0.00 \\
\hline 2 & Rro & 1 & 10 & 60 & 40 & 8.46 & 0.00 \\
\hline 3 & Ruu & 1 & 8 & 62.5 & 37.5 & 7.93 & 0.00 \\
\hline 4 & Rhd & 1 & 20 & 65 & 35 & 7.4 & 0.00 \\
\hline 5 & Rku & 1 & 87 & 73.56 & 26.44 & 5.59 & 0.00 \\
\hline 6 & Rav & 1 & 33 & 75.76 & 24.24 & 5.13 & 0.00 \\
\hline 7 & Ros & 1 & 225 & 88.44 & 11.56 & 2.44 & 0.00 \\
\hline & & & 4336 & 95.27 & 4.73 & 1 & \\
\hline
\end{tabular}

Hip Analysis

In this portion of the results section, a similar series of results to the knee analysis are presented. Here, two sets of results are again produced: (a) All IVs and (b) Comorbidity IVs. The hip data set contains the same eight administrative All IVs but a different set of Comorbidity IVs.

- All IVs

- Coarse Searches (Models without Loops)

The hip data set, with 3,205 cases, originally had 170 Comorbidity IVs. A loopless search for individually predictive variables provided the rationale in reducing the total 
Comorbidity IVs. Individually predictive variables were kept in addition to the

Comorbidity IVs that were found to be predictive in the literature. The resulting data set

consisted of 45 variables. The top 10 predicting variables are below in Table 34 .

Table 34. Summary of Search Results for All IVs for DV Complication (Hip). Search covers directed coarse and fine models. All p values $=0$.

\begin{tabular}{|c|c|c|c|c|}
\hline MODEL & $\Delta \mathrm{df}$ & $\Delta \mathrm{BIC}$ & $\% \Delta \mathrm{H}$ & Variable description \\
\hline \multicolumn{5}{|c|}{ COARSE, single predictors (top 10) } \\
\hline $\mathrm{Nrb} \mathrm{Cp}$ & 2 & 70.88 & 6.72 & Number of risks (binned) \\
\hline $\mathrm{S} \mathrm{Cp}$ & 42 & -272.10 & 5.17 & Surgeon \\
\hline $\mathrm{Ad} \mathrm{Cp}$ & 38 & -246.01 & 4.69 & Admit diagnosis \\
\hline Ageb Cp & 2 & 38.11 & 4.19 & Age (binned) \\
\hline Rrd Cp & 1 & 38.60 & 3.61 & Unspecified hypertensive renal disease (403.9) \\
\hline $\mathrm{Fc} \mathrm{Cp}$ & 5 & 1.35 & 3.22 & Financial class \\
\hline Rca Cp & 1 & 27.91 & 2.78 & $\begin{array}{l}\text { Coronary atherosclerosis of native coronary artery } \\
(414.01)\end{array}$ \\
\hline Ruh Cp & 1 & 27.11 & 2.72 & Other and unspecified hyperlipidemia (272.4) \\
\hline $\mathrm{Rku} \mathrm{Cp}$ & 1 & 21.67 & 2.30 & Chronic kidney disease, unspecified (585.9) \\
\hline $\mathrm{Rpl} \mathrm{Cp}$ & 1 & 19.16 & 2.10 & Hyperplasia of prostate (600) \\
\hline MODEL & $\Delta \mathrm{df}$ & $\Delta \mathrm{BIC}$ & $\% \Delta \mathrm{H}$ & Variable description \\
\hline \multicolumn{5}{|c|}{ COARSE, IVs in AIC or BIC models but not in top 10} \\
\hline Rhd Cp (rank 11) & 1 & 14.76 & 1.76 & Other chronic pulmonary heart disease (416.8) \\
\hline Rgp Cp (rank 25) & 1 & -0.25 & 0.60 & Repair of cystocele with graft or prosthesis (70.54) \\
\hline MODEL & $\Delta \mathrm{df}$ & $\triangle \mathrm{BIC}$ & $\% \Delta \mathrm{H}$ & Variable description \\
\hline \multicolumn{5}{|c|}{ COARSE, best model (loopless) } \\
\hline \multicolumn{5}{|c|}{$\Delta \mathrm{BIC}$ (best model) } \\
\hline Nrb Rrd Cp & 5 & 71.05 & 8.61 & $\begin{array}{l}\text { Number of risks (binned), Unspecified hypertensive } \\
\text { renal disease (403.9) }\end{array}$ \\
\hline \multicolumn{5}{|c|}{ Inc.P \& $\triangle \mathrm{AIC}$ (same best model) } \\
\hline Ageb Nrb Cp & 2 & 66.21 & 10.10 & Age (binned), Number of risks (binned) \\
\hline MODEL & $\Delta \mathrm{df}$ & $\Delta \mathrm{BIC}$ & $\% \Delta H$ & Variable description \\
\hline \multicolumn{5}{|c|}{ FINE, best models (with loops) } \\
\hline \multicolumn{5}{|l|}{$\Delta \mathrm{BIC}($ best model) } \\
\hline $\begin{array}{l}\text { Ageb Cp : Nrb Cp : Rrd Cp : } \\
\text { Rca Cp : Rhd Cp : Rpl Cp }\end{array}$ & 8 & 109.68 & 13.46 & $\begin{array}{l}\text { Age (binned), Number of risks (binned), Unspecified } \\
\text { hypertensive renal disease (403.9), Coronary } \\
\text { atherosclerosis of native coronary artery }(414.01) \text {, } \\
\text { Other chronic pulmonary heart diseases }(416.8) \text {, } \\
\text { Hyperplasia of prostate }(600)\end{array}$ \\
\hline \multicolumn{5}{|c|}{ Inc.P \& $\triangle \mathrm{AIC}$ (same best model) } \\
\hline $\begin{array}{l}\text { Ageb Cp :Nrb Cp : Rrd Cp : } \\
\text { Rca Cp : Rhd Cp : Rpl Cp : } \\
\operatorname{Rgp~Cp}\end{array}$ & 9 & 109.23 & 14.05 & $\begin{array}{l}\text { Age (binned), Number of risks (binned), Unspecified } \\
\text { hypertensive renal disease (403.9), Coronary } \\
\text { atherosclerosis of native coronary artery (414.01), } \\
\text { Other chronic pulmonary heart diseases (416.8), } \\
\text { Hyperplasia of prostate (600), Repair of cystocele } \\
\text { with graft or prosthesis }(70.54)\end{array}$ \\
\hline
\end{tabular}


Looking at the single predicting variables, simply knowing the total number of comorbidities a patient had (Nrb) reduces the uncertainty in predicting Complication (Cp) by $6.72 \%$. Knowing only the surgeon (S) that performed the surgery reduces uncertainty by $5.17 \%$, admit diagnosis (Ad) by $4.69 \%$ and age (Ageb) by $4.19 \%$. The first predictive individual comorbidity is hypertensive renal disease (Rrd) which reduces uncertainty by $3.61 \%$, and so on. The best loopless coarse model shows that knowing both Nrb and Rrd reduces the uncertainty by $8.61 \%$.

- Fine Searches (Models with Loops)

As with the knee analysis, a fine-grained search has allowed for multiple components in the prediction of Complication (Cp). While this type of search allows for the detection of interaction effects, none were discovered in the best models for this search. There are 6 single predicting variables in the best BIC fine-grained model Ageb $\mathbf{C p}: \operatorname{Nrb} \mathbf{C p}: \operatorname{Rrd} \mathbf{C p}: \operatorname{Rca} \mathbf{C p}$ : Rhd $\mathbf{C p}: \operatorname{Rpl} \mathbf{C p}$. Five of these variables are in the top 10 as seen in Table 41 above, and the sixth, Rhd, is the 11th single predicting variable. While one might expect to see the more predictive of the single predicting variables included in the best fine-grained BIC model, the variables surgeon (S), admit diagnosis (Ad), financial class $(\mathrm{Fc})$ and the two Comorbidity IVs hyperlipidemia (Ruh) and chronic kidney disease (Rku) did not make it into the best BIC model. Any predictive effects offered by these variables may overlap with those of the variables already in the model, and so be excluded, or the effects may not improve the model enough to balance the cost that each incurs in increased complexity. The next variable that added new information was chronic pulmonary heart disease (Rhd). 


\section{- Comorbidity IVs}

- Coarse Searches (Models without Loops)

The most predictive Comorbidity IVs (top 10) are listed below in Table 35, which shows the search results including only the Comorbidity IVs. The comorbidities are in the same order as in Table 34 but show additional predictive comorbidities that were in a sense hidden by the more predictive All IVs Ageb and Nrb.

Table 35. Summary of Search Results for Comorbidity IVs (Hip). Search covers directed coarse, and fine models.

\begin{tabular}{|c|c|c|c|c|c|}
\hline MODEL & $\Delta \mathrm{df}$ & $\Delta \mathrm{BIC}$ & $\% \Delta \mathrm{H}$ & Alpha & Variable description \\
\hline \multicolumn{6}{|l|}{$\begin{array}{l}\text { COARSE, single } \\
\text { predictors }\end{array}$} \\
\hline Rrd Cp & 1 & 38.60 & 3.61 & 0.00 & Unspecified hypertensive renal disease (403.9) \\
\hline Rca Cp & 1 & 27.91 & 2.78 & 0.00 & $\begin{array}{l}\text { Coronary atherosclerosis of native coronary artery } \\
(414.01)\end{array}$ \\
\hline Ruh Cp & 1 & 27.11 & 2.72 & 0.00 & Other and unspecified hyperlipidemia (272.4) \\
\hline Rku Cp & 1 & 21.67 & 2.30 & 0.00 & Chronic kidney disease, unspecified (585.9) \\
\hline Rpl Cp & 1 & 19.16 & 2.10 & 0.00 & Hyperplasia of prostate $(600)$ \\
\hline Rhd Cp & 1 & 14.76 & 1.76 & 0.00 & Other chronic pulmonary heart diseases (416.8) \\
\hline Rdi Cp & 1 & 13.79 & 1.69 & 0.00 & Diabetes mellitus (250) \\
\hline Rkd Cp & 1 & 11.98 & 1.55 & 0.00 & $\begin{array}{l}\text { Chronic kidney disease, Stage III (moderate) } \\
(585.3)\end{array}$ \\
\hline Raf Cp & 1 & 11.35 & 1.50 & 0.00 & Atrial fibrillation $(427.31)$ \\
\hline Rck Cp & 1 & 6.55 & 1.13 & 0.00 & Anemia in chronic kidney disease (285.21) \\
\hline MODEL & $\Delta \mathrm{df}$ & $\Delta \mathrm{BIC}$ & $\% \Delta \mathrm{H}$ & Alpha & Variable description \\
\hline \multicolumn{6}{|c|}{ COARSE, IVs in AIC or BIC models but not in top 10} \\
\hline Rhe Cp (rank 12) & 1 & 4.52 & 0.97 & 0.00 & Unspecified essential hypertension (401.9) \\
\hline Rhh Cp (rank15) & 1 & 2.96 & 0.85 & 0.00 & Hyposmolality and/or hyponatremia (276.1) \\
\hline Ram Cp (rank 17) & 1 & 1.81 & 0.76 & 0.00 & Unspecified deficiency anemia (281.9) \\
\hline Rgp Cp (rank 19) & 1 & -0.25 & 0.60 & 0.00 & Repair of cystocele with graft or prosthesis (70.54) \\
\hline Rfr Cp (rank 23) & 1 & -1.02 & 0.54 & 0.01 & Nonunion of fracture $(733.82)$ \\
\hline Rra Cp (rank 26) & 1 & -1.68 & 0.49 & 0.01 & Alcohol abuse, in remission (305.03) \\
\hline MODEL & $\Delta \mathrm{df}$ & $\Delta \mathrm{BIC}$ & $\% \Delta \mathrm{H}$ & Alpha & Variable description \\
\hline \multicolumn{6}{|l|}{$\begin{array}{l}\text { COARSE, best model } \\
\text { (loopless) }\end{array}$} \\
\hline \multicolumn{6}{|l|}{$\Delta B I C$ (best model) } \\
\hline Rrd Rca Cp & 3 & 52.96 & 5.96 & 0.00 & $\begin{array}{l}\text { Unspecified hypertensive renal disease ( } 403.9) \text {, } \\
\text { Coronary atherosclerosis of native coronary artery } \\
(414.01) \text {, }\end{array}$ \\
\hline \multicolumn{6}{|l|}{$\Delta \mathrm{AIC}$ (best model) } \\
\hline Ruh Rrd Rca Rpl Cp & 15 & 8.98 & 10.05 & 0.00 & $\begin{array}{l}\text { Other and unspecified hyperlipidemia (272.4), } \\
\text { Unspecified hypertensive renal disease (403.9), } \\
\text { Coronary atherosclerosis of native coronary artery } \\
\text { (414.01), Hyperplasia of prostate (600) }\end{array}$ \\
\hline
\end{tabular}




\begin{tabular}{|c|c|c|c|c|c|}
\hline \multicolumn{6}{|l|}{ Inc.P (best model) } \\
\hline Ruh Rrd Rca Raf Rpl Cp & 31 & -93.15 & 12.14 & 0.01 & $\begin{array}{l}\text { Other and unspecified hyperlipidemia (272.4), } \\
\text { Unspecified hypertensive renal disease (403.9), } \\
\text { Coronary atherosclerosis of native coronary artery } \\
\text { (414.01), Atrial fibrillation (427.31), Hyperplasia } \\
\text { of prostate (600) }\end{array}$ \\
\hline MODEL & $\Delta \mathrm{df}$ & $\triangle \mathrm{BIC}$ & $\% \Delta \mathrm{H}$ & Alpha & Variable description \\
\hline \multicolumn{6}{|l|}{$\begin{array}{l}\text { FINE, best models } \\
\text { (with loops) }\end{array}$} \\
\hline \multicolumn{6}{|l|}{$\Delta B I C$ (best model) } \\
\hline $\begin{array}{l}\text { Rdi Cp : Ruh Cp : Rhh } \\
\text { Cp : Ram Cp : Rra Cp : } \\
\text { Rhe Cp : Rrd Cp : Rca } \\
\text { Cp : Rhd Cp : Rpl Cp : } \\
\text { Rgp Cp : Rfr Cp }\end{array}$ & 12 & 101.79 & 15.35 & 0.00 & $\begin{array}{l}\text { Diabetes mellitus (250), Other and unspecified } \\
\text { hyperlipidemia (272.4), Hyposmolality and/or } \\
\text { hyponatremia (276.1), Unspecified deficiency } \\
\text { anemia (281.9), Alcohol abuse, in remission } \\
\text { (305.03), Unspecified essential hypertension } \\
\text { (401.9), Unspecified hypertensive renal disease } \\
\text { (403.9), Coronary atherosclerosis of native } \\
\text { coronary artery (414.01), Other chronic } \\
\text { pulmonary heart diseases (416.8), Hyperplasia of } \\
\text { prostate (600), Repair of cystocele with graft or } \\
\text { prosthesis (70.54), Nonunion of fracture (733.82) }\end{array}$ \\
\hline \multicolumn{6}{|c|}{ Inc.P \& $\triangle \mathrm{AIC}$ (same best model) } \\
\hline $\begin{array}{l}\text { Rdi Cp : Ruh Cp : Rhh } \\
\text { Cp : Ram Cp : Rra Cp : } \\
\text { Rhe Cp : Rrd Cp : Rca } \\
\text { Cp : Rhd Cp : Raf Cp : } \\
\text { Rpl Cp : Rgp Cp : Rfr } \\
\text { Cp }\end{array}$ & 13 & 100.13 & 15.84 & 0.01 & $\begin{array}{l}\text { Diabetes mellitus (250), Other and unspecified } \\
\text { hyperlipidemia (272.4), Hyposmolality and/or } \\
\text { hyponatremia (276.1), Unspecified deficiency } \\
\text { anemia (281.9), Alcohol abuse, in remission } \\
\text { (305.03), Unspecified essential hypertension } \\
\text { (401.9), Unspecified hypertensive renal disease } \\
\text { (403.9), Coronary atherosclerosis of native } \\
\text { coronary artery (414.01), Other chronic } \\
\text { pulmonary heart diseases (416.8), Atrial } \\
\text { fibrillation (427.31), Hyperplasia of prostate } \\
\text { (600), Repair of cystocele with graft or prosthesis } \\
\text { (70.54), Nonunion of fracture (733.82) }\end{array}$ \\
\hline
\end{tabular}

With the All IVs included, the best coarse model by the BIC criterion was Nrb Rrd Cp, which had a $\% \Delta \mathrm{H}$ of 8.61 . The best coarse model in the Comorbidity IVs search is Rrd Rca Cp, with a \% $\% \mathrm{H}$ of 5.96. Hypertensive renal disease ( $\mathrm{Rrd})$ in fact is the top single predicting comorbidity in both hip and knee searches, and is included in the best BIC model for loopless searches. In the knee data set, Ruh was predictive and was included in the best loopless model along with Rrd. Just as in the knee data set, in the hip data set Ruh is the third most predictive single variable. In the hip search results Ruh is 
not selected by BIC but rather is selected by the AIC criterion as a predicting component in the loopless search. More comparisons between the hip and knee data sets within the DV are provided at the end of this section.

\section{- Fine Searches (Models with Loops)}

In the search that allows loops, the best model by BIC with Comorbidity IVs resulted in Rdi Cp : Ruh $\mathbf{C p}: \operatorname{Rhh} \mathbf{C p}: \operatorname{Ram} \mathbf{C p}: \operatorname{Rra} \mathbf{C p}: \operatorname{Rhe} \mathbf{C p}: \operatorname{Rrd} \mathbf{C p}: \operatorname{Rca} \mathbf{C p}:$ Rhd Cp : Rpl Cp : Rgp Cp : Rfr Cp with a \% $\%$ H of 15.35 (Table 35), a large improvement over the loopless model in this Comorbidity IVs search, and slight improvement over the best model from the prior search that included All IVs which had a $\% \Delta \mathrm{H}$ of 13.46 (Table 34 ). This best model does not have any interaction terms, and the comorbidities are all individually predictive of $\mathrm{Cp}$. Overall, however, there is a type of interaction effect — not the familiar kind — due to the combination of the multiple components of the model (Zwick, 2011c). Each of these IVs, diabetes mellitus (Rdi) other and unspecified hyperlipidemia (Ruh), hyposmolality and/or hyponatremia (Rhh), unspecified deficiency anemia (Ram), alcohol abuse, in remission (Rra), unspecified essential hypertension (Rhe), unspecified hypertensive renal disease (Rrd), coronary atherosclerosis of native coronary artery (Rca), other chronic pulmonary heart diseases (Rhd), hyperplasia of prostate (Rpl), repair of cystocele with graft or prosthesis $(\mathrm{Rgp})$, nonunion of fracture (Rfr), is examined in detail in the model's joint conditional probability distribution in Table 43. 


\section{- Comparing Search Results (Hip, Cp)}

All IVs and the Comorbidity IVs searches yield two sets of results that can be compared to each other and interpreted into a three-tiered classification of predicting variables, as summarized in Table 36.

Table 36. The 3-Tiered Classification of Predicting Variables for DV: Complication, Hip Analysis.

\begin{tabular}{|l|l|}
\hline Tier & Variables \\
\hline Tier 1 (Most Important) & Ageb, Nrb, Rrd, Rca, Rhd, Rpl \\
\hline Tier 2 & Rgp \\
\hline Tier 3 & Rdi, Ruh, Rhh, Ram, Rra, Rhe, Rfr \\
\hline
\end{tabular}

Tier 1 contains variables from the best-by-BIC model from the dataset All IVs. This is the most complete search, and the one that provides the most conservative predictors. The selected variables (Ageb, Nrb, Rrd, Rca, Rhd, Rpl) are shown in the first row of Table 36, and are considered the most important predicting variables.

Tier 2 contains variables not in Tier 1, but found in the AIC (less conservative) model of All IVs AND in the BIC model of Comorbidity IVs. For the Hip Analysis of DV Complication, this selects the variable Rgp as the next-most important predicting variable as shown in the second row of Table 36.

Finally, this classification places into Tier 3 any variables present elsewhere in the best model search results, but not included in Tier 1 or Tier 2 . That is, any variables unique to one of the two searches: variables in the AIC model of All IVs but not in the BIC model of Comorbidity IVs, and variables in the BIC model of Comorbidity IVs but not in the AIC model from All IVs. These variables (Rdi, Ruh, Rhh, Ram, Rra, Rhe, Rfr) are in the last row of Table 36. 
- Model FIT

The detailed content of the best fine-grained BIC model from the All IVs search, Ageb $\mathbf{C p}: \operatorname{Nrb} \mathbf{C p}: \operatorname{Rrd} \mathbf{C p}: \operatorname{Rca} \mathbf{C p}: \operatorname{Rhd} \mathbf{C p}: \operatorname{Rpl} \mathbf{C p}$, is shown as a conditional probability distribution in Table 37 below. This joint probability distribution contains many fewer rows than the distribution from the best BIC model from the knee data in Table 24, because rows have been excluded that either are not significant $(\mathrm{p}>0.05)$ or have too low a frequency $(<10)$, to focus on more informative results.

Table 37. Fit Table (Hip) for All IVs for Best Model Ageb Cp : Nrb Cp : Rrd Cp : Rca Cp : Rhd Cp : Rpl Cp.

Blue rows indicate ratio $<0.90$ and orange rows indicate ratio $>1.10$. (Ratios between 0.91 and 1.09 are close to the margins and are excluded.)

\begin{tabular}{|c|c|c|c|c|c|c|c|c|c|c|c|c|c|c|}
\hline & \multicolumn{10}{l|}{ IVs } & \multicolumn{10}{l|}{ Data } & \multicolumn{3}{l|}{ Model } \\
\hline & & & & & & & & \multicolumn{2}{l|}{ obs. p(DV|IV) } & \multicolumn{2}{c|}{ calc. q(DV|IV) } & \multicolumn{3}{l|}{} \\
\hline$\#$ & Ageb & Nrb & Rrd & Rca & Rhd & Rpl & freq & $\mathrm{Cp}=0$ & $\mathrm{Cp}=1$ & $\mathrm{Cp}=0$ & $\mathrm{Cp}=1$ & Ratio & $\mathrm{p}$ (margin) \\
\hline 1 & 1 & 1 & 0 & 0 & 0 & 0 & 475 & 97.90 & 2.11 & 98.49 & 1.52 & 0.30 & 0.00 \\
\hline 3 & 1 & 2 & 0 & 0 & 0 & 0 & 348 & 97.70 & 2.30 & 98.27 & 1.73 & 0.34 & 0.00 \\
\hline 13 & 2 & 1 & 0 & 0 & 0 & 0 & 355 & 98.03 & 1.97 & 98.25 & 1.76 & 0.34 & 0.00 \\
\hline 16 & 2 & 2 & 0 & 0 & 0 & 0 & 337 & 97.63 & 2.37 & 98.00 & 2.00 & 0.39 & 0.01 \\
\hline 37 & 3 & 3 & 0 & 0 & 0 & 0 & 312 & 87.82 & 12.18 & 89.66 & 10.34 & 2.02 & 0.00 \\
\hline 38 & 3 & 3 & 0 & 0 & 0 & 1 & 24 & 83.33 & 16.67 & 71.68 & 28.32 & 5.53 & 0.00 \\
\hline 40 & 3 & 3 & 0 & 1 & 0 & 0 & 55 & 78.18 & 21.82 & 79.84 & 20.17 & 3.94 & 0.00 \\
\hline 43 & 3 & 3 & 1 & 0 & 0 & 0 & 31 & 70.97 & 29.03 & 72.42 & 27.58 & 5.39 & 0.00 \\
\hline 46 & 3 & 3 & 1 & 1 & 0 & 0 & 11 & 54.55 & 45.46 & 54.53 & 45.47 & 8.89 & 0.00 \\
\hline & & & & & & & 3205 & 94.88 & 5.12 & 94.88 & 5.12 & 1.00 & \\
\hline
\end{tabular}

The values for the All IVs: number of risks binned (Nrb) and age binned (Ageb) are the variables that number of risks and age were discretized to with possible states being either in bin 1,2, or 3 . The bin ranges and frequencies are summarized in Table 38 and Table 39 . 
Table 38. Number of Risks Binned (Nrb)

Bin Range \& Frequency (Hip).

\begin{tabular}{|c|c|c|}
\hline Bin & Range & Frequency \\
\hline 1 & $0-1$ & 1,111 \\
\hline 2 & $2-3$ & 1,081 \\
\hline 3 & $4-19$ & 1,013 \\
\hline
\end{tabular}

Table 39. Age Binned (Ageb) Bin Range \& Frequency (Hip).

\begin{tabular}{|c|c|c|}
\hline Bin & Range & Frequency \\
\hline 1 & $15-59$ & 1,090 \\
\hline 2 & $60-69$ & 1,027 \\
\hline 3 & $70-96$ & 1,088 \\
\hline
\end{tabular}

Each of the component's individual projections is informative, yet not as informative as the full model's joint distribution (Table 37). In the component table below (Table 40) for Ageb $=2$, the probability of $\mathbf{C p}$ was $3.51 \%$ with a risk ratio of 0.68 . In the model's full joint distribution in row 13, when Ageb $=2$ and when Nrb was its lowest in bin 1 and the comorbidities in the model are all absent, then the probability of a complication is essentially cut in half at $1.76 \%$, with a risk ratio of 0.34 . Row 16 shows almost the same scenario; however, with Nrb slightly higher $(\mathrm{Nrb}=2)$, the probability of Cp of $2 \%$ was still lower than the overall data (the margins), with a ratio of 0.39 . Once Ageb and Nrb get to their highest states, we see increased risk (rows 37, 38, 40, 43, 46). Row 37 shows that when Ageb and Nrb were both in bin 3, and the individual comorbidities were absent, the risk of $\mathbf{C p}$ increased to $10.34 \%$, much higher than the complication rate for the whole sample, with a risk ratio of 2.02. Introduce the presence of Rpl (hyperplasia of prostate), and the risk more than doubles, with a probability of $\mathbf{C p}=28.32 \%$ and a ratio of 5.53 (row 38). With Ageb and Nrb each in bin 3, the presence of Rca (coronary atherosclerosis of native coronary artery) alone nearly doubled the risk 
to $20.17 \%$, with a ratio of 3.94 (row 40). The presence of just Rrd (hypertensive renal disease) with Ageb and Nrb each in bin 3 increased risk of $\mathbf{C p}$ to $7.58 \%$ with a ratio of 5.39 (row 43). When either hyperplasia of prostate (Rpl), coronary atherosclerosis of native coronary artery (Rca), or hypertensive renal disease (Rrd) were present along with high Ageb and a higher total number of risks in total (Nrb), then the probability of developing a complication increased at least four times that of the probability of entire sample. Row 46 shows what happens when an additional comorbidity, Rca, is present in the model when Ageb $=3, \mathrm{Nrb}=3$, and Rrd is present. In cases where these states occur, the patient had a $45.47 \%$ (ratio 8.89) probability of Complication (Cp) occurring.

The decision tree version of the conditional probability distribution provides a different perspective of the same results (Figure 8). 


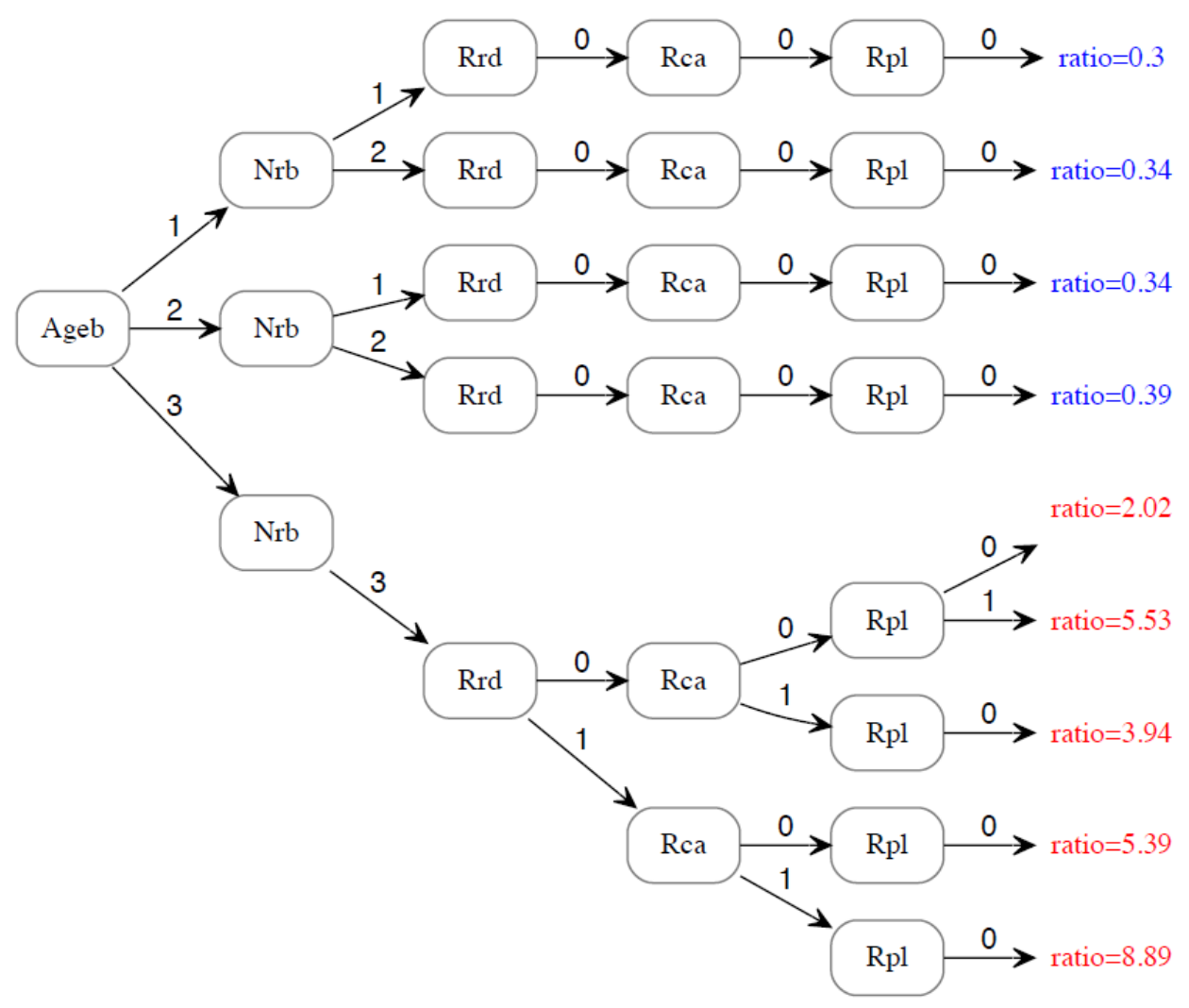

Figure 8. Decision Tree for DV Any Comp (Hip) All IVs for Best Model Ageb Cp : Nrb Cp : Rrd Cp : Rca Cp : Rhd Cp : Rpl Cp.

Each component of the model has its own individual probability distribution.

Table 40 shows the conditional probability of $\mathbf{C p}=1$ given Ageb $=1$ is $2.57 \%$, with a risk ratio of 0.50; when $\mathrm{Ageb}=2$, the probability of $\mathbf{C p}=1$ is $3.51 \%$; and when Ageb $=3$, the risk increased with the probability of $\mathbf{C p}=1$ at $9.19 \%$, with a risk ratio of 1.80. As was seen with the knee data, lower age offered a protective effect while higher age increased risk of Cp. Additional model components are listed in Table 41 and Table 42, but are self-explanatory. 
Table 40. Component Fit Table for IVAgeb in (Hip) All IVs Best Model: Ageb Cp : Nrb Cp : Rrd Cp : Rca Cp : Rhd Cp : Rpl Cp.

Blue rows indicate ratio $<0.90$ and orange rows indicate ratio $>1.10$.

\begin{tabular}{|c|c|c|c|c|c|c|}
\hline & IV & Data & \multicolumn{2}{|c|}{ obs. $\mathrm{p}(\mathrm{DV} \mid \mathrm{IV})$} & & \\
\hline$\#$ & Ageb & freq & $\mathrm{Cp}=0$ & $\mathrm{Cp}=1$ & ratio & $\mathrm{p}$ (margin) \\
\hline 1 & 1 & 1090 & 97.43 & 2.57 & 0.50 & 0.00 \\
\hline 2 & 2 & 1027 & 96.50 & 3.51 & 0.68 & 0.02 \\
\hline 3 & 3 & 1088 & 90.81 & 9.19 & 1.80 & 0.00 \\
\hline & & 3205 & 94.88 & 5.12 & 1.00 & \\
\hline
\end{tabular}

Table 41. Component Fit Table for IV Nrb in (Hip) All IVs Best Model: Ageb Cp : Nrb Cp : Rrd Cp : Rca Cp : Rhd Cp : Rpl Cp.

Blue rows indicate ratio $<0.90$ and orange rows indicate ratio $>1.10$.

\begin{tabular}{|c|c|c|c|c|c|c|}
\hline & IV & Data & \multicolumn{2}{|c|}{ obs. $\mathrm{p}(\mathrm{DV} \mid \mathrm{IV})$} & & \\
\hline$\#$ & Nrb & freq & $\mathrm{Cp}=0$ & $\mathrm{Cp}=1$ & ratio & $\mathrm{p}$ (margin) \\
\hline 1 & 1 & 1111 & 97.84 & 2.16 & 0.42 & 0.00 \\
\hline 2 & 2 & 1081 & 97.04 & 2.96 & 0.58 & 0.00 \\
\hline 3 & 3 & 1013 & 89.34 & 10.66 & 2.08 & 0.00 \\
\hline & \multicolumn{7}{|c|}{3205} & 94.88 & 5.12 & 1.00 & \\
\hline
\end{tabular}

Table 42. Condensed Component Fit Table for IVs Rhd, Rrd, Rpl and Rca in (Hip) All IVs Best Model: Ageb Cp : Nrb Cp : Rrd Cp : Rca Cp : Rhd Cp : Rpl Cp.

Blue rows indicate ratio $<0.90$ and orange rows indicate ratio $>1.10$.

(Ratios between 0.91 and 1.09 are close to the margins and are excluded.)

\begin{tabular}{|c|c|c|c|c|c|c|}
\hline & & Data & \multicolumn{2}{|c|}{ obs. $\mathrm{p}(\mathrm{DV} \mid \mathrm{IV})$} & & \\
\hline IV & IV States & freq & $\mathrm{Cp}=0$ & $\mathrm{Cp}=1$ & Ratio & $\mathrm{p}$ (margin) \\
\hline Rhd & 1 & 10 & 40.00 & 60.00 & 11.73 & 0.00 \\
\hline Rrd & 0 & 3128 & 95.46 & 4.54 & 0.89 & 0.14 \\
\hline Rrd & 1 & 77 & 71.43 & 28.57 & 5.58 & 0.00 \\
\hline Rpl & 1 & 77 & 77.92 & 22.08 & 4.31 & 0.00 \\
\hline Rca & 0 & 3039 & 95.56 & 4.44 & 0.87 & 0.09 \\
\hline Rca & 1 & 166 & 82.53 & 17.47 & 3.41 & 0.00 \\
\hline & & 3205 & 94.88 & 5.12 & 1.00 & \\
\hline
\end{tabular}

The joint probability distribution below in Table 43 show the details for the best fine model from the hip Comorbidity IVs search Rdi Cp : Ruh Cp : Rhh Cp : Ram Cp : Rra Cp : Rhe Cp : Rrd Cp : Rca Cp : Rhd Cp : Rpl Cp : Rgp Cp : Rfr Cp. In this distribution, each of the IVs are either 0 (comorbidity absent) or 1 (comorbidity present). Row 1 is the case where all of the comorbidities included in the model are absent, which 
lowers the risk of Complication (Cp) from 5.12\% (the model's marginal probability) to $1.92 \%$, with a ratio of 0.37 .

Table 43. Fit Table (Hip) for Comorbidity IVs for Best Model Rdi Cp : Ruh Cp : Rhh Cp : Ram Cp : Rra Cp : Rhe Cp : Rrd Cp : Rca Cp : Rhd Cp : Rpl Cp : Rgp Cp : Rfr Cp. Blue rows indicate ratio $<0.90$ and orange rows indicate ratio $>1.10$. (Ratios between 0.91 and 1.09 are close to the margins and are excluded.) (Variables Rhh, Ram, Rra, Rhd, Rgp, and Rfr only take value 0 for these rows.)

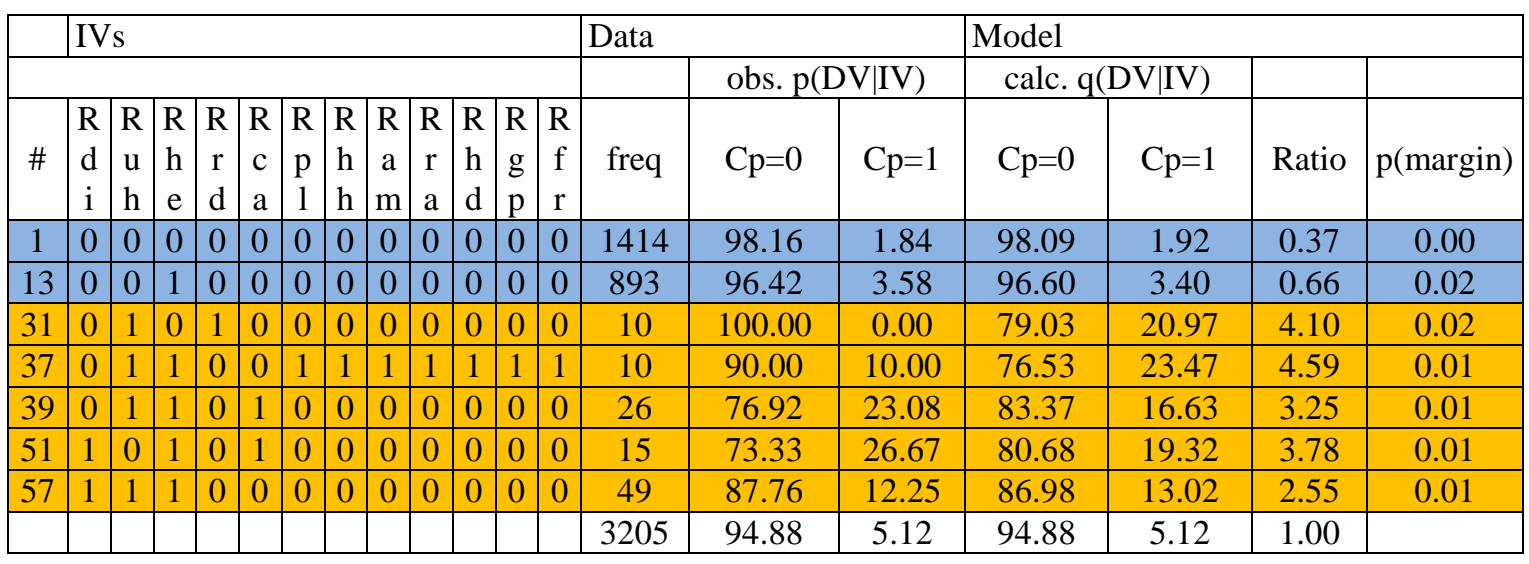

Having only the comorbidity Rhe (essential hypertension) still keeps the risk lower, with a ratio of 0.66 (row 13). However, with the additional presence of either Rdi (diabetes mellitus), Ruh (hyperlipidemia), Rca (coronary atherosclerosis of native coronary artery) or Rpl (Hyperplasia of prostate), the risk increases. Row 37 shows that in addition to the presence of Rhe (essential hypertension), the presence of Ruh (hyperlipidemia) and Rpl (Hyperplasia of prostate) increases risk of $\mathbf{C p}$ to $23.47 \%$ (ratio $=4.59$ ). Row 39 shows that with Rhe and Ruh present, but with the additional Rca comorbidity, the probability of $\mathbf{C p}=16.63 \%$ (ratio $=3.25)$. Row 51 shows that with Rhe present again, but this time along with Rdi and Rca, the probability of $\mathbf{C p}=19.32 \%$ (ratio $=3.78$ ), and with Rhe and Rdi both present, but with Ruh, the probability of $\mathbf{C p}=13.0 \%($ ratio $=2.55)$. Risk is also increased over Hypertension alone when even a single other comorbidity is present, as with diabetes mellitus (Rdi), hyperlipidemia (Ruh), 
coronary atherosclerosis of native coronary artery (Rca), and/or hyperplasia of prostate (Rpl). For these cases, the risk of Complication (Cp) increases from the marginal probability of $5.12 \%$ to between $13.02 \%$ and $23.47 \%$.

The decision tree for the conditional probability distribution for model Rdi Cp : Ruh Cp : Rhh Cp : Ram Cp : Rra Cp : Rhe Cp : Rrd Cp : Rca Cp : Rhd Cp : Rpl Cp : $\operatorname{Rgp} \mathbf{C p}$ : Rfr $\mathbf{C p}$ (Figure 9) offers perhaps a simplified way of looking at how the presence or absence of each Comorbidity IV, and their combinations, lead to increased or decreased risk of Complication (Cp).

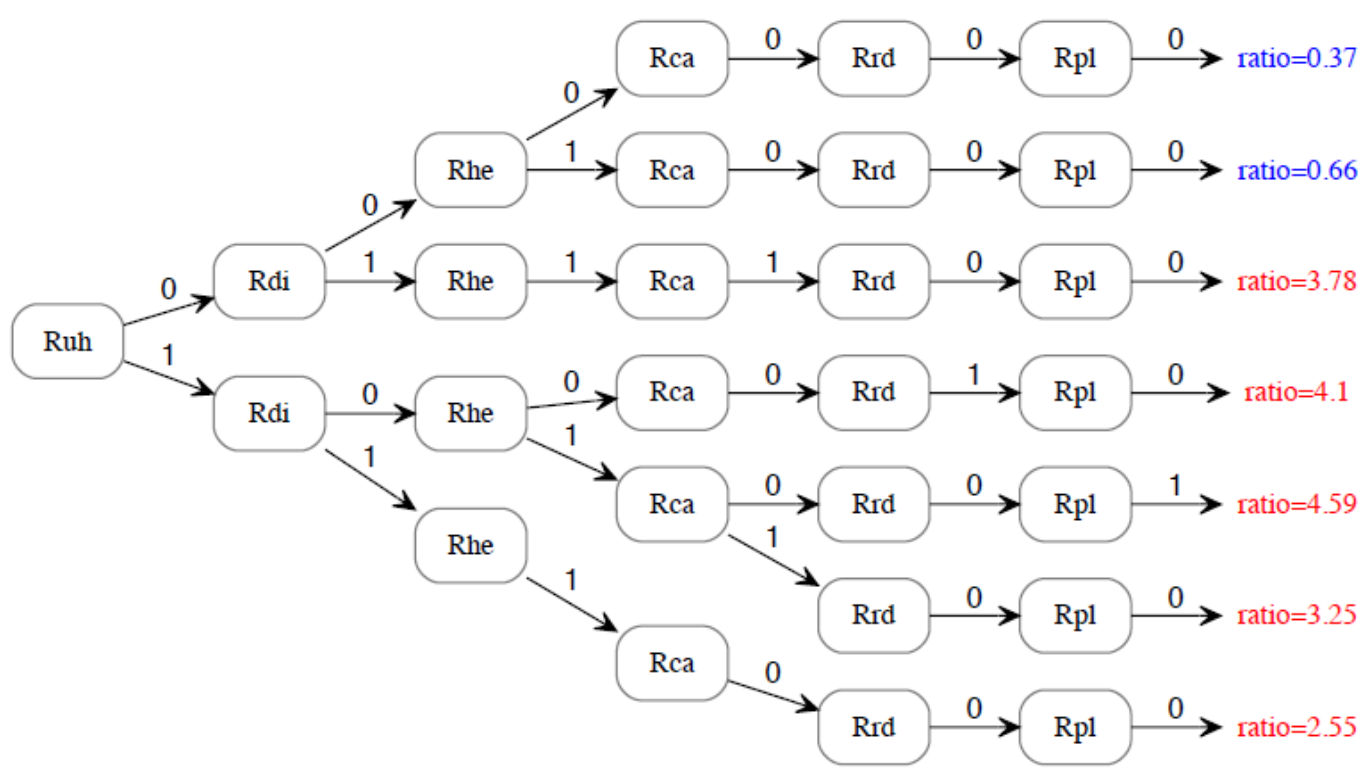

Figure 9. Decision Tree for DV Any Comp (Hip) with Comorbidity IVs for Best Model Rdi Cp : Ruh Cp : Rhh Cp : Ram Cp : Rra Cp : Rhe Cp : Rrd Cp : Rca Cp : Rhd Cp : Rpl Cp : Rgp Cp : Rfr Cp.

The individual components of the model each have a fit table, condensed into a single table below (Table 44). 
Table 44. Condensed Component Fit Table for IVs Rhd, Ram, Rhh, Rgp, Rfr, Rra, Rrd, and Rpl in (Hip) Comorbidity IVs Best Model: Rdi Cp : Ruh Cp : Rhh Cp : Ram Cp : Rra Cp : Rhe Cp : Rrd Cp : Rca Cp : Rhd Cp : Rpl Cp : Rgp Cp : Rfr Cp. Orange rows indicate ratio $>1.10$.

(Ratios between 0.91 and 1.09 are close to the margins and are excluded.)

\begin{tabular}{|c|c|c|c|c|c|c|c|}
\hline & & & Data & \multicolumn{2}{|c|}{ obs. p(DV|IV) } & & \\
\hline$\#$ & IV & IV State & freq & $\mathrm{Cp}=0$ & $\mathrm{Cp}=1$ & Ratio & $\mathrm{p}$ (margin) \\
\hline 1 & Rhd & 1 & 10 & 40.00 & 60.00 & 11.73 & 0.00 \\
\hline 2 & Ram & 1 & 6 & 50.00 & 50.00 & 9.77 & 0.00 \\
\hline 3 & Rhh & 1 & 10 & 60.00 & 40.00 & 7.82 & 0.00 \\
\hline 4 & Rgp & 1 & 8 & 62.50 & 37.50 & 7.33 & 0.00 \\
\hline 5 & Rfr & 1 & 9 & 66.67 & 33.33 & 6.51 & 0.00 \\
\hline 6 & Rra & 1 & 10 & 70.00 & 30.00 & 5.86 & 0.00 \\
\hline 7 & Rrd & 1 & 77 & 71.43 & 28.57 & 5.58 & 0.00 \\
\hline 8 & Rpl & 1 & 77 & 77.92 & 22.08 & 4.31 & 0.00 \\
\hline 9 & Rca & 1 & 166 & 82.53 & 17.47 & 3.41 & 0.00 \\
\hline 10 & Rdi & 1 & 305 & 88.53 & 11.48 & 2.24 & 0.00 \\
\hline 11 & Ruh & 1 & 466 & 88.63 & 11.37 & 2.22 & 0.00 \\
\hline 12 & Rhe & 1 & 1464 & 93.37 & 6.63 & 1.29 & 0.01 \\
\hline & & & 3205 & 94.88 & 5.12 & 1.00 & \\
\hline
\end{tabular}

Hip \& Knee Summary of Results for Complication

The most important IVs are summarized in Table 45. The IVs that show up in both searches are number of risks (Nrb), age (Ageb), chronic pulmonary heart disease (Rhd), and hyperlipidemia (Ruh).

Table 45. Summary of Most Important IVs by Tier across Hip and Knee for Complication.

\begin{tabular}{|c|l|l|}
\hline & \multicolumn{2}{|c|}{ Complication (Cp) } \\
\hline Tier & \multicolumn{1}{|c|}{ Knee } & \multicolumn{1}{c|}{ Hip } \\
\hline 1 & Nrb, Ageb, Ruh, Rhd, Rku & Ageb, Nrb, Rrd, Rca, Rhd, Rpl \\
\hline 2 & Reg, Raf, Ros, Rav, Ruu & Rgp \\
\hline 3 & Rro & Rdi, Ruh, Rhh, Ram, Rra, Rhe, Rfr \\
\hline
\end{tabular}

\section{DV: Skilled Nursing Facility (SNF)}

Knee Analysis

In this results section, the analysis is conducted with the same knee data set as with DV Complication (Cp), but using the DV Skilled Nursing Facility (SNF) instead. 
SNF is a binary variable, with a patient either being discharged to a SNF or not. SNF occurs much more frequently in the data than DV Cp, with approximately $17 \%$ of patients discharged to a SNF after a knee replacement compared to the occurrence of DV Cp of 4.7\%. In this analysis, a new series of best models, whose measure of goodness is also $\%$ reduction of uncertainty, are described and analyzed in detail. This analysis also looks at (a) All IVs, and (b) only the Comorbidity IVs.

(This SNF analysis results section is briefer than that provided above for DV Cp, with less discussion of the methodology. To the extent that each results section uses the same methodology, the detailed discussion given for $\mathbf{C p}$ can be used for reference, as can the RA methodology portion of the Methods chapter.)

- All IVs

- Coarse Searches (Models without Loops)

The final knee data set for SNF contained 67 IVs. The variable reduction method of performing a loopless search for single predicting variables found 55 IVs to be individually predictive of $\mathbf{S N F}$ with $\mathrm{p} \leq 0.05$. Of these $55 \mathrm{IVs}, 12$ were also found to be predictive of similar outcomes in the literature. However, the literature had found 12 IVs to be predictive that were not found to be individually predictive in the loopless search conducted in this project. These $12 \mathrm{IVs}$ were retained in the knee data set for this analysis.

In the prior analysis section, the search to predict Complication $(\mathbf{C p})$ in the knee data found the best single predictor to be surgeon (S) with a $\% \Delta \mathrm{H}$ of 6.45 . The top single predicting IV of $\mathbf{S N F}$ is financial class $(\mathrm{Fc})$ with a $\% \Delta \mathrm{H}$ of 10.55 (Table 46). The top 10 
single predicting IVs for SNF are listed below in Table 46 and are ordered smallest to largest for alpha, which is the inverse of $\% \Delta \mathrm{H}$.

Age (Ageb) reduces uncertainty of SNF by $10.5 \%$ and by simply knowing the surgeon $(\mathrm{S})$ there is a $\% \Delta \mathrm{H}$ of 7.18 . Number of risks $(\mathrm{Nrb})$ has a $\% \Delta \mathrm{H}$ of 2.57 , hospital location (L) a $\% \Delta \mathrm{H}$ of 2.14 , and admit diagnosis (Ad) a $\% \Delta \mathrm{H}$ of 1.03 . The first individual patient Comorbidity IV that is predictive of $\mathbf{S N F}$ is heart failure (Rhf), with a $\% \Delta \mathrm{H}$ of 0.95 , followed by glaucoma (Rug), with a value of 0.9 , and then persistent mental disorders (Rmd) at 0.85 . 
Table 46. Summary of Search Results for Knee data, DV SNF. Includes both All IVs for coarse and fine models.

\begin{tabular}{|c|c|c|c|c|c|}
\hline MODEL & $\Delta \mathrm{df}$ & $\triangle \mathrm{BIC}$ & $\% \Delta \mathrm{H}$ & Alpha & Variable description \\
\hline \multicolumn{6}{|c|}{ COARSE, single predictors (top 10) } \\
\hline Fc SNF & 5 & 383.00 & 10.55 & 0.00 & Financial class \\
\hline Ageb SNF & 2 & 406.13 & 10.50 & 0.00 & Age binned \\
\hline S SNF & 62 & -229.95 & 7.18 & 0.00 & Surgeon \\
\hline Nrb SNF & 2 & 86.87 & 2.57 & 0.00 & Number of risks binned \\
\hline L SNF & 6 & 35.81 & 2.14 & 0.00 & Location \\
\hline Ad SNF & 27 & -184.59 & 1.03 & 0.04 & Admit diagnosis \\
\hline Rhf SNF & 1 & 29.93 & 0.95 & 0.00 & Heart Failure (428) \\
\hline Rug SNF & 1 & 27.95 & 0.90 & 0.00 & Unspecified glaucoma (365.9), \\
\hline Rmd SNF & 1 & 26.03 & 0.85 & 0.00 & Persistent mental disorders (294.8) \\
\hline Svb SNF & 2 & 15.18 & 0.79 & 0.00 & Surgeon volume binned \\
\hline MODEL & $\Delta \mathrm{df}$ & $\Delta \mathrm{BIC}$ & $\% \Delta \mathrm{H}$ & Alpha & Variable description \\
\hline \multicolumn{6}{|c|}{ COARSE, IVs in AIC or BIC models but not in top 10} \\
\hline Rpa SNF (rank 21) & 1 & 2.99 & 0.28 & 0.00 & Parkinson's disease (332) \\
\hline Rbp SNF (rank 26) & 1 & 1.06 & 0.23 & 0.00 & Other and unspecified bipolar disorders (296.8) \\
\hline MODEL & $\Delta \mathrm{df}$ & $\Delta \mathrm{BIC}$ & $\% \Delta \mathrm{H}$ & Alpha & Variable description \\
\hline \multicolumn{6}{|c|}{ COARSE, best model (loopless) } \\
\hline \multicolumn{6}{|c|}{$\Delta \mathrm{BIC}$ (best model) } \\
\hline Ageb Nrb SNF & 8 & 451.49 & 12.87 & 0.00 & Age binned + Number of risks binned \\
\hline \multicolumn{6}{|c|}{$\triangle$ AIC \& Inc.P (same best model) } \\
\hline AgebNrbRmdSNF & 17 & 401.43 & 13.50 & 0.00 & $\begin{array}{l}\text { Age binned + Number of risks binned + persistent } \\
\text { mental disorders (294.8) }\end{array}$ \\
\hline MODEL & $\Delta \mathrm{df}$ & $\Delta \mathrm{BIC}$ & $\% \Delta \mathrm{H}$ & Alpha & Variable description \\
\hline \multicolumn{6}{|c|}{ FINE, best models (with loops) } \\
\hline \multicolumn{6}{|c|}{$\Delta \mathrm{BIC}$ (best model) } \\
\hline $\begin{array}{l}\text { L SNF : Fc SNF : } \\
\text { Ageb SNF : Svb } \\
\text { SNF : Nrb SNF : } \\
\text { Rmd SNF: Rug } \\
\text { SNF: Rhf SNF }\end{array}$ & 20 & 635.69 & 19.94 & 0.00 & $\begin{array}{l}\text { location, financial class, age binned, surgeon } \\
\text { volume binned, number of risks binned, persistent } \\
\text { mental disorders (294.8), Unspecified glaucoma } \\
\text { (365.9), Heart Failure (428) }\end{array}$ \\
\hline \multicolumn{6}{|c|}{ Inc.P \& $\triangle \mathrm{AIC}$ (same best model) } \\
\hline $\begin{array}{l}\text { L SNF : Fc SNF : } \\
\text { Ageb SNF : Svb } \\
\text { SNF : Nrb SNF } \\
\text { :Rmd SNF: Rbp } \\
\text { SNF : Rpa SNF : } \\
\text { Rug SNF : Rhf } \\
\text { SNF }\end{array}$ & 22 & 634.61 & 20.33 & 0.00 & $\begin{array}{l}\text { location, financial class, age binned, surgeon } \\
\text { volume binned, number of risks binned, persistent } \\
\text { mental disorders (294.8), Other and unspecified } \\
\text { bipolar disorders (296.8), Parkinson's disease } \\
\text { (332), Unspecified glaucoma (365.9), Heart Failure } \\
\text { (428) }\end{array}$ \\
\hline
\end{tabular}

The best coarse model allowing for more than one IV (but not allowing for loops)

is Ageb $\mathrm{Nrb}$ SNF, with a corresponding $\% \Delta \mathrm{H}$ of 12.87 and a $\Delta \mathrm{df}$ of 8 . Financial class

$(\mathrm{Fc})$, while individually predictive, is not included as it does not provide additional

information worth the complexity over the combined IVs of Ageb and Nrb. Ageb and 
$\mathrm{Nrb}$ form a conventional interaction effect that is different from the simple combination of two separate components (i.e., Ageb SNF : Nrb SNF).

- Fine Searches (Models with Loops)

Allowing for multiple components and loops, the best model by BIC found 8 IVs in the model L SNF : Fc SNF : Ageb SNF : Svb SNF : Nrb SNF : Rmd SNF : Rug SNF : Rhf SNF. All 8 of these IVs are also listed in the top 10 single predicting IVs of Table 46. Similar to the search results for $\mathbf{C p}$, surgeon (S) is individually predictive but does not appear in the best fine-grained model, presumably because of the high cardinality of S (63 states). Location (L), financial class ( $\mathrm{Fc})$, age binned (Ageb), surgeon volume binned $(\mathrm{Svb})$, number of risks binned $(\mathrm{Nrb})$, persistent mental disorders (Rmd), unspecified glaucoma (Rug), and heart failure (Rhf) reduce uncertainty of SNF by $19.94 \%$ with a $\Delta$ df of 20 .

Both the coarse and fine-grained searches were dominated by the effects of the non-comorbidity variables present in All IVs. In order to look only at the effect of the patient's individual comorbidities, a Comorbidity IVs search is performed.

- Comorbidity IVs

- Coarse Searches (Models without Loops)

Considering only Comorbidity IVs as predictors, the most predictive individual comorbidities are uncovered, yet no single comorbidity has a $\% \Delta \mathrm{H}$ over 1 (Table 47 ). Individual comorbidities alone do not seem very predictive of discharge to SNF. 
Table 47. Summary of Search Results (Knee) for Comorbidity IVs for SNF. Search covers directed coarse, and fine models.

\begin{tabular}{|c|c|c|c|c|c|}
\hline MODEL & $\Delta \mathrm{df}$ & $\Delta \mathrm{BIC}$ & $\% \Delta \mathrm{H}$ & Alpha & Variable description \\
\hline \multicolumn{6}{|l|}{ COARSE, single predictors } \\
\hline Rhf SNF & 1 & 29.93 & 0.95 & 0.00 & Heart failure (428) \\
\hline Rug SNF & 1 & 27.95 & 0.90 & 0.00 & Unspecified glaucoma (365.9), \\
\hline Rmd SNF & 1 & 26.03 & 0.85 & 0.00 & Persistent mental disorders (294.8) \\
\hline Rku SNF & 1 & 21.81 & 0.75 & 0.00 & Chronic kidney disease (585.9) \\
\hline Rrd SNF & 1 & 19.04 & 0.68 & 0.00 & $\begin{array}{l}\text { Unspecified hypertensive renal disease } \\
(403.9)\end{array}$ \\
\hline Rbn SNF & 1 & 16.49 & 0.62 & 0.00 & Other disorders of bone and cartilage (733) \\
\hline Rin SNF & 1 & 13.77 & 0.55 & 0.00 & Urinary incontinence (788.3) \\
\hline Rhy SNF & 1 & 12.34 & 0.51 & 0.00 & Hypothyroidism (244.9) \\
\hline Rhd SNF & 1 & 9.67 & 0.45 & 0.00 & $\begin{array}{l}\text { Other chronic pulmonary heart diseases } \\
(416.8)\end{array}$ \\
\hline Rau SNF & 1 & 9.04 & 0.43 & 0.00 & Anemia, unspecified (285.9) \\
\hline MODEL & $\Delta \mathrm{df}$ & $\Delta \mathrm{BIC}$ & $\% \Delta \mathrm{H}$ & Alpha & Variable description \\
\hline \multicolumn{6}{|c|}{ COARSE, IVs in AIC or BIC models but not in top 10} \\
\hline Rhe SNF (rank 14) & 1 & 5.06 & 0.33 & 0.00 & Essential hypertension (401.9) \\
\hline Rpa SNF (rank 15) & 1 & 2.99 & 0.28 & 0.00 & Parkinson's disease (332) \\
\hline MODEL & $\Delta \mathrm{df}$ & $\Delta \mathrm{BIC}$ & $\% \Delta \mathrm{H}$ & & Variable description \\
\hline \multicolumn{6}{|c|}{ COARSE, best model (loopless) } \\
\hline \multicolumn{6}{|c|}{$\Delta \mathrm{BIC}$ (best model) } \\
\hline RugRhf SNF & 3 & 50.72 & 1.88 & 0.00 & $\begin{array}{l}\text { Unspecified glaucoma }(365.9)+\text { Heart } \\
\text { Failure }(428)\end{array}$ \\
\hline \multicolumn{6}{|l|}{$\Delta \mathrm{AIC}$ (best model) } \\
\hline RmdRugRhfRku SNF & 15 & 9.20 & 3.35 & 0.00 & $\begin{array}{l}\text { persistent mental disorders (294.8) + } \\
\text { Unspecified glaucoma (365.9) + Heart } \\
\text { Failure }(428)+\text { Chronic kidney disease } \\
(585.9)\end{array}$ \\
\hline \multicolumn{6}{|l|}{ Inc.P (best model) } \\
\hline RmdRugRhfRkuRbn SNF & 31 & -98.97 & 3.99 & 0.02 & $\begin{array}{l}\text { persistent mental disorders }(294.8)+ \\
\text { Unspecified glaucoma }(365.9)+\text { Heart } \\
\text { Failure }(428)+\text { Chronic kidney disease } \\
(585.9)+\text { Other disorders of bone and } \\
\text { cartilage (733) }\end{array}$ \\
\hline MODEL & $\Delta \mathrm{df}$ & $\Delta \mathrm{BIC}$ & $\% \Delta \mathrm{H}$ & & Variable description \\
\hline \multicolumn{6}{|c|}{ FINE, best models (with loops) } \\
\hline \multicolumn{6}{|c|}{$\Delta \mathrm{BIC}$, Inc.P \& $\triangle \mathrm{AIC}$ (same best model) } \\
\hline $\begin{array}{l}\text { Rhy SNF : Rau SNF : } \\
\text { Rmd SNF : Rpa SNF : } \\
\text { Rug SNF : Rhe SNF : } \\
\text { Rhf SNF : Rku : } \\
\text { Rbn SNF : Rin SNF }\end{array}$ & 10 & 138.61 & 5.52 & 0.00 & $\begin{array}{l}\text { Hypothyroidism (244.9), Anemia (285.9), } \\
\text { persistent mental disorders (294.8), } \\
\text { Parkinson's disease (332), Unspecified } \\
\text { glaucoma (365.9), Essential hypertension } \\
\text { (401.9), Heart failure (428), Chronic } \\
\text { kidney disease (585.9), Other disorders of } \\
\text { bone and cartilage (733), Urinary } \\
\text { incontinence (788.3) }\end{array}$ \\
\hline
\end{tabular}


The results of a coarse search allowing for multiple IVs yields a best BIC model Rug Rhf SNF. Together, Rug and Rhf have a $\% \Delta H$ of 1.88 , close to what adding their separate effects of 0.9 and 0.95 would give.

○ Fine Searches (Models with Loops)

In a search that allows for loops, the best BIC model is Rhy SNF : Rau SNF : Rmd SNF : Rpa SNF : Rug SNF : Rhe SNF : Rhf SNF : Rku SNF : Rbn SNF : Rin SNF, with a corresponding reduction in uncertainty of $5.52 \%$ and a $\Delta \mathrm{df}$ of 10 . Hypothyroidism (Rhy), anemia (Rau), persistent mental disorders (Rmd), Parkinson's disease (Rpa), unspecified glaucoma (Rug), essential hypertension (Rhe), heart failure (Rhf), chronic kidney disease ( $\mathrm{Rku})$, other disorders of bone and cartilage (Rbn), and urinary incontinence (Rin) are not nearly as predictive as the model that allowed for All IVs.

\section{- Comparing Search Results (Knee, SNF)}

All IVs and the Comorbidity IVs searches yield two sets of results that can be compared to each other and interpreted into a three-tiered classification of predicting variables, as shown in Table 48.

Table 48. The 3-Tiered Classification of Predicting Variables for DV SNF, Knee Analysis.

\begin{tabular}{|l|l|}
\hline Tier & Variables \\
\hline Tier 1 (Most Important) & L, Fc, Ageb, Svb, Nrb, Rmd, Rug, Rhf \\
\hline Tier 2 & Rpa \\
\hline Tier 3 & Rbp, Rhy, Rau, Rhe, Rku, Rbn, Rin \\
\hline
\end{tabular}

As with earlier analyses, the Tier 1 variables are those from the BIC model from All IVs. This is the most complete and conservative search, and these are deemed the most important predicting variables: L, Fc, Ageb, Svb, Nrb, Rmd, Rug, and Rhf. 
The Tier 2 variables are those not in Tier 1, but still found in the best models of both searches: the AIC (less conservative) model of All IVs, and the BIC model of Comorbidity IVs. This selects Rpa as the best IV by Tier 2 .

Finally, Tier 3 contains the variables in only one of the best models (the same as with Tier 2), but not in both. For DV SNF, these lowest-tier predicting variables are: Rbp, Rhy, Rau, Rhe, Rku, Rbn, and Rin.

\section{- Model FIT}

The best fine-grained model, L SNF : Fc SNF : Ageb SNF : Svb SNF : Nrb SNF : Rmd SNF : Rug SNF : Rhf SNF, is explained in detail by the joint conditional probability distribution in Table 49 below. 
Table 49. Fit Table (Knee) for All IVs for Best Model L SNF : Fc SNF : Ageb SNF : Svb SNF : Nrb SNF : Rmd SNF : Rug SNF : Rhf SNF. Blue rows indicate ratio $<0.90$ and orange rows indicate ratio $>$ 1.10. (Ratios between 0.91 and 1.09 are close to the margins and are excluded.)

\begin{tabular}{|c|c|c|c|c|c|c|c|c|c|c|c|c|c|c|c|}
\hline \multicolumn{9}{|c|}{ IVs } & \multicolumn{3}{|l|}{ Data } & \multicolumn{4}{|c|}{ Model } \\
\hline & & & & & & & & & & \multicolumn{2}{|c|}{ obs. p(DV|IV) } & \multicolumn{3}{|c|}{ calc. $\mathrm{q}(\mathrm{DV} \mid \mathrm{IV})$} & \multirow[b]{2}{*}{$p$ (margin) } \\
\hline$\#$ & \begin{tabular}{|l|l}
$\mathrm{L} \mid \mathrm{I}$ \\
$\mathrm{s}$
\end{tabular} & $\mathrm{ac}$ & Ageb & Svb & $\mathrm{Nrb}$ & Rmd & Rug & Rhf & freq & $\mathrm{SNF}=1$ & $\mathrm{SNF}=2$ & $\mathrm{SNF}=1$ & $\mathrm{SNF}=2$ & Ratio & \\
\hline 1 & 1 & 2 & 1 & 2 & 1 & 0 & 0 & 0 & $55^{1}$ & 100.00 & 0.00 & 97.89 & 2.11 & 0.12 & 0.00 \\
\hline 2 & 2 & 1 & 2 & 1 & 3 & 0 & 0 & 0 & 41 & 78.05 & 21.95 & 66.44 & 33.56 & 1.91 & 0.01 \\
\hline 3 & 2 & 1 & 2 & 2 & 3 & 0 & 0 & 0 & 52 & 63.46 & 36.54 & 66.08 & 33.92 & 1.93 & 0.00 \\
\hline 5 & 2 & 1 & 3 & 1 & 1 & 0 & 0 & 0 & 20 & 70.00 & 30.00 & 64.65 & 35.35 & 2.01 & 0.04 \\
\hline 6 & 2 & 1 & 3 & 1 & 2 & 0 & 0 & 0 & 62 & 66.13 & 33.87 & 55.08 & 44.92 & 2.56 & 0.00 \\
\hline 7 & 2 & 1 & 3 & 1 & 3 & 0 & 0 & 0 & 41 & 46.34 & 53.66 & 44.47 & 55.53 & 3.16 & 0.00 \\
\hline 11 & 2 & 1 & 3 & 2 & 1 & 0 & 0 & 0 & 30 & 63.33 & 36.67 & 64.28 & 35.73 & 2.04 & 0.01 \\
\hline 12 & 2 & 1 & 3 & 2 & 2 & 0 & 0 & 0 & 62 & 51.61 & 48.39 & 54.68 & 45.32 & 2.58 & 0.00 \\
\hline 14 & 2 & 1 & 3 & 2 & 3 & 0 & 0 & 0 & 70 & 47.14 & 52.86 & 44.07 & 55.93 & 3.19 & 0.00 \\
\hline 18 & 2 & 1 & 3 & 3 & 2 & 0 & 0 & 0 & 134 & 68.66 & 31.34 & 69.97 & 30.04 & 1.71 & 0.00 \\
\hline 19 & 2 & 1 & 3 & 3 & 3 & 0 & 0 & 0 & 84 & 64.29 & 35.71 & 60.34 & 39.66 & 2.26 & 0.00 \\
\hline 22 & 2 & 2 & 1 & 1 & 1 & 0 & 0 & 0 & 49 & 97.96 & 2.04 & 96.38 & 3.62 & 0.21 & 0.01 \\
\hline 23 & 2 & 2 & 1 & 1 & 2 & 0 & 0 & 0 & 61 & 93.44 & 6.56 & 94.70 & 5.30 & 0.30 & 0.01 \\
\hline 24 & 2 & 2 & 1 & 2 & 1 & 0 & 0 & 0 & 55 & 98.18 & 1.82 & 96.33 & 3.67 & 0.21 & 0.01 \\
\hline 25 & 2 & 2 & 1 & 2 & 2 & 0 & 0 & 0 & 77 & 96.10 & 3.90 & 94.62 & 5.38 & 0.31 & 0.01 \\
\hline 26 & 2 & 2 & 1 & 2 & 3 & 0 & 0 & 0 & 68 & 89.71 & 10.29 & 91.99 & 8.01 & 0.46 & 0.04 \\
\hline 27 & 2 & 2 & 1 & 3 & 1 & 0 & 0 & 0 & 119 & 99.16 & 0.84 & 98.06 & 1.94 & 0.11 & 0.00 \\
\hline 28 & 2 & 2 & 1 & 3 & 2 & 0 & 0 & 0 & 118 & 95.76 & 4.24 & 97.14 & 2.86 & 0.16 & 0.00 \\
\hline 29 & 2 & 2 & 1 & 3 & 3 & 0 & 0 & 0 & 73 & 93.15 & 6.85 & 95.69 & 4.32 & 0.25 & 0.00 \\
\hline 30 & 2 & 2 & 2 & 3 & 1 & 0 & 0 & 0 & 35 & 97.14 & 2.86 & 97.06 & 2.94 & 0.17 & 0.03 \\
\hline 31 & 2 & 2 & 2 & 3 & 2 & 0 & 0 & 0 & 55 & 92.73 & 7.27 & 95.67 & 4.33 & 0.25 & 0.01 \\
\hline 33 & 3 & 1 & 3 & 1 & 3 & 0 & 0 & 0 & 84 & 58.33 & 41.67 & 70.84 & 29.16 & 1.66 & 0.01 \\
\hline 36 & 3 & 1 & 3 & 2 & 3 & 0 & 0 & 0 & 55 & 70.91 & 29.09 & 70.51 & 29.49 & 1.68 & 0.02 \\
\hline 39 & 3 & 2 & 1 & 1 & 1 & 0 & 0 & 0 & 40 & 100.00 & 0.00 & 98.78 & 1.22 & 0.07 & 0.01 \\
\hline 40 & 3 & 2 & 1 & 1 & 2 & 0 & 0 & 0 & 63 & 96.83 & 3.18 & 98.19 & 1.81 & 0.10 & 0.00 \\
\hline 41 & 3 & 2 & 1 & 1 & 3 & 0 & 0 & 0 & 49 & 100.00 & 0.00 & 97.25 & 2.75 & 0.16 & 0.01 \\
\hline 42 & 3 & 2 & 1 & 2 & 1 & 0 & 0 & 0 & 22 & 100.00 & 0.00 & 98.76 & 1.24 & 0.07 & 0.04 \\
\hline 43 & 3 & 2 & 1 & 2 & 2 & 0 & 0 & 0 & 34 & 100.00 & 0.00 & 98.16 & 1.84 & 0.10 & 0.02 \\
\hline 44 & 3 & 2 & 1 & 2 & 3 & 0 & 0 & 0 & 32 & 100.00 & 0.00 & 97.21 & 2.79 & 0.16 & 0.03 \\
\hline 45 & 3 & 2 & 1 & 3 & 1 & 0 & 0 & 0 & 21 & 100.00 & 0.00 & 99.35 & 0.65 & 0.04 & 0.05 \\
\hline 46 & 3 & 2 & 1 & 3 & 2 & 0 & 0 & 0 & 23 & 100.00 & 0.00 & 99.04 & 0.96 & 0.05 & 0.04 \\
\hline 47 & 3 & 2 & 1 & 3 & 3 & 0 & 0 & 0 & 37 & 100.00 & 0.00 & 98.54 & 1.47 & 0.08 & 0.01 \\
\hline 48 & 4 & 1 & 2 & 2 & 3 & 0 & 0 & 0 & 24 & 83.33 & 16.67 & 63.34 & 36.66 & 2.09 & 0.01 \\
\hline 52 & 4 & 1 & 3 & 2 & 1 & 0 & 0 & 0 & 21 & 76.19 & 23.81 & 61.47 & 38.53 & 2.20 & 0.01 \\
\hline 53 & 4 & 1 & 3 & 2 & 2 & 0 & 0 & 0 & 25 & 36.00 & 64.00 & 51.69 & 48.31 & 2.75 & 0.00 \\
\hline 54 & 4 & 1 & 3 & 2 & 3 & 0 & 0 & 0 & 46 & 34.78 & 65.22 & 41.14 & 58.86 & 3.35 & 0.00 \\
\hline 56 & 6 & 1 & 2 & 1 & 1 & 0 & 0 & 0 & 37 & 94.60 & 5.41 & 94.70 & 5.30 & 0.30 & 0.05 \\
\hline & & & & & & & & & 4336 & 82.45 & 17.55 & 82.45 & 17.55 & 1.00 & \\
\hline$\#$ & \begin{tabular}{ll|l}
$\mathrm{L}$ & $\mathrm{I}$ \\
$\mathrm{H}$
\end{tabular} & \begin{tabular}{ll|}
$\mathrm{Fc}$ & \\
\end{tabular} & Ageb & $\mathrm{Svb}$ & $\mathrm{Nrb}$ & Rmd & Rug & Rhf & freq & $\mathrm{SNF}=1$ & $\mathrm{SNF}=2$ & $\mathrm{SNF}=1$ & $\mathrm{SNF}=2$ & Ratio & $\mathrm{p}($ margin $)$ \\
\hline
\end{tabular}

Financial class $(\mathrm{Fc})$ at first seemed that it might be redundant with age. However, financial class $=1$ (Medicare) can cover patients who are age 65 and over, or those 
younger than 65 and receiving disability benefits. Moreover, the age bins do not line up along age requirements for Medicare, and the highest age bin has a minimum age of 72 (Table 50).

Table 50. Age bin distribution.

\begin{tabular}{|c|c|c|}
\hline Bin & Range & Frequency \\
\hline 1 & $32-62$ & 1,490 \\
\hline 2 & $63-71$ & 1,411 \\
\hline 3 & $72-95$ & 1,435 \\
\hline
\end{tabular}

There is some association between financial class $(\mathrm{Fc})$ and age binned (Ageb), which is supported by the finding that when $\mathrm{Fc}$ is made the DV, its single best predictor is Ageb with $\% \Delta \mathrm{H}(\mathrm{Fc})$ of 38.37 . However, the best model selected to predict SNF includes both $\mathrm{Fc}$ and Ageb, which indicates that each of these IVs offers additional information worth the added complexity.

For the calculated model, knowing the states of All IVs (L, Fc, Ageb, Svb, Nrb) or the presence or absence of the individual Comorbidity IVs (Rmd, Rug, Rhf) tells us about the probability of SNF occurring. Each of the model's components, namely L SNF or Fc SNF or Ageb SNF or Svb SNF or Nrb SNF or Rmd SNF or Rug SNF or Rhf SNF, has an individual conditional probability distribution and is individually informative. For example, looking only at location (L) (Table 51), the conditional probability of SNF given location 1 is $12.3 \%$, with the risk ratio of 0.70 . Additional locations that seem with low projected discharges to SNF include locations 3, 6, and 7, with risk ratios 0.69, 0.62, and 0.17 . Locations 2 and 4 show increased risk with ratios (1.15 and 1.67 respectively). Location 5 has a neutral risk ratio, with the projected discharge to SNF similar to that of the observed rate in the data with a risk ratio of 0.96 . In other words, knowing only the 
location of the surgery, regardless of the states of the other IVs in the model, there is a significant difference in the probability of $\mathbf{S N F}=\mathbf{2}$ between the locations.

Table 51. Component Fit Table for IV $\mathrm{L}$ in (Knee) All IVs Best Model L SNF : Fc SNF : Ageb SNF : Svb SNF : Nrb SNF : Rmd SNF : Rug SNF : Rhf SNF. Blue rows indicate ratio $<0.90$ and orange rows indicate ratio $>1.10$. (Ratios between 0.91 and 1.09 are close to the margins and are excluded.)

\begin{tabular}{|c|c|c|c|c|c|c|}
\hline & IV & Data & \multicolumn{2}{|c|}{ obs. p(DV|IV) } & & \\
\hline$\#$ & L & freq & SNF=1 & SNF=2 & Ratio & p(margin) \\
\hline 1 & 1 & 244 & 87.71 & 12.30 & 0.70 & 0.03 \\
\hline 2 & 2 & 2143 & 79.75 & 20.25 & 1.15 & 0.00 \\
\hline 3 & 3 & 1138 & 87.87 & 12.13 & 0.69 & 0.00 \\
\hline 4 & 4 & 349 & 70.77 & 29.23 & 1.67 & 0.00 \\
\hline 6 & 6 & 267 & 89.14 & 10.86 & 0.62 & 0.00 \\
\hline 7 & 7 & 34 & 97.06 & 2.94 & 0.17 & 0.03 \\
\hline & & 4336 & 82.45 & 17.55 & 1.00 & \\
\hline
\end{tabular}

Knowing only the financial class $(\mathrm{Fc})$ is individually informative as well.

Financial class 1 is Medicare, and patients with Medicare have $26.89 \%$ chance of discharging to SNF compared to $17.55 \%$ overall, with a risk ratio of 1.53 . Patients with financial class 2 (commercial payers) have a very low projection of discharging to a $\mathbf{S N F}$ at $4.4 \%$ and a risk ratio of 0.25 . Patients with financial class 3 (Medicaid) had an increased risk of SNF at 21.13\%, with a ratio of 1.2. Both financial classes 4 and 5 (Workers Comp and Other Government) offered decreased risk of SNF with risk ratios of 0.19 and 0 . Perhaps financial class 4 and 5 payers did not offer a skilled nursing facility benefit, something worth looking into in any future confirmatory analysis. The individual component table for Fc is below in Table 52, while the remainder of the individual component tables are available in Appendix I: Supplementary Tables. 
Table 52. Component Fit Table for IV Fc in (Knee) All IVs Best Model

L SNF : Fc SNF : Ageb SNF : Svb SNF : Nrb SNF : Rmd SNF : Rug SNF : Rhf SNF.

Blue rows indicate ratio $<0.90$ and orange rows indicate ratio $>1.10$.

\begin{tabular}{|c|c|c|c|c|c|c|}
\hline & IV & Data & \multicolumn{2}{|c|}{ obs. p(DV|IV) } & & \\
\hline$\#$ & Fc & freq & SNF=1 & SNF=2 & Ratio & p(margin) \\
\hline 1 & 1 & 2484 & 73.11 & 26.89 & 1.53 & 0.00 \\
\hline 2 & 2 & 1727 & 95.60 & 4.40 & 0.25 & 0.00 \\
\hline 3 & 3 & 71 & 78.87 & 21.13 & 1.20 & 0.43 \\
\hline 4 & 4 & 30 & 96.67 & 3.33 & 0.19 & 0.04 \\
\hline 5 & 5 & 17 & 100.00 & 0.00 & 0.00 & 0.07 \\
\hline 6 & 6 & 7 & 85.71 & 14.29 & 0.81 & 0.83 \\
\hline & & 4336 & 82.45 & 17.55 & 1.00 & \\
\hline
\end{tabular}

The cardinality of the All IVs for this knee analysis search and fit for SNF are many-valued, with seven possible states for location (L), six possible states for financial class $(\mathrm{Fc})$, and three possible states each for age binned (Ageb), surgeon volume binned (Svb), and number of risks binned (Nrb). Therefore, the combinations of IVs that are summarized above in Table 49 resulted in a very large decision tree. However, one can determine blue or orange by looking at $\mathrm{Fc}$ alone. For $\mathrm{Fc}=1$, one gets orange. For $\mathrm{Fc}=2$, one gets blue. There is only one exception to this in line 56, which has Fc $=1$ and is blue (Table 49). Decision trees have two purposes in the present context: (1) to distinguish coarsely between blue and orange (between decreased risk and increased risk IV states), and (2) to distinguish more finely between degrees of protectiveness or riskiness, as shown by the ratio values.

The details from the best fine model from the Comorbidity IVs search, Rhy SNF : Rau SNF : Rmd SNF : Rpa SNF : Rug SNF : Rhe SNF : Rhf SNF : Rku SNF : Rbn SNF : Rin SNF, are provided in Table 53 below and also in the decision tree in Figure 10 below. Again, each comorbidity is either present or absent. The absence of all of the comorbidities offers a protective effect against SNF with a risk ratio of 0.66. 
With only the presence of essential hypertension (Rhe), there is still a slight protective effect with ratio $=0.90$.

Table 53. Fit Table (Knee) for Comorbidity IVs, for Best Model, Rhy SNF : Rau SNF : Rmd SNF : Rpa SNF : Rug SNF : Rhe SNF : Rhf SNF : Rku SNF : Rbn SNF : Rin SNF. Blue rows indicate ratio $<0.90$ and orange rows indicate ratio $>1.10$.

(Ratios between 0.91 and 1.09 are close to the margins and are indicated in gray.)

\begin{tabular}{|c|c|c|c|c|c|c|c|c|c|c|c|c|c|c|c|c|c|}
\hline & IV & & & & & & & & & & & Data & & Model & & & \\
\hline & & & & & & & & & & & & obs. & $\mathrm{V} \mid \mathrm{IV})$ & calc. & $\mathrm{V} \mid \mathrm{IV})$ & & \\
\hline \# & \begin{tabular}{|l}
$\mathrm{R}$ \\
$\mathrm{h}$ \\
$\mathrm{y}$
\end{tabular} & $\begin{array}{l}\mathrm{R} \\
\mathrm{a} \\
\mathrm{u}\end{array}$ & $\begin{array}{l}\mathrm{R} \\
\mathrm{m} \\
\mathrm{d}\end{array}$ & $\begin{array}{l}\mathrm{R} \\
\mathrm{p} \\
\mathrm{a}\end{array}$ & $\begin{array}{l}\mathrm{R} \\
\mathrm{u} \\
\mathrm{g}\end{array}$ & $\begin{array}{l}\mathrm{R} \\
\mathrm{h} \\
\mathrm{e}\end{array}$ & $\begin{array}{l}\mathrm{R} \\
\mathrm{h} \\
\mathrm{f}\end{array}$ & $\begin{array}{l}\mathrm{R} \\
\mathrm{k} \\
\mathrm{u}\end{array}$ & $\begin{array}{l}\mathrm{R} \\
\mathrm{b} \\
\mathrm{n}\end{array}$ & $\begin{array}{c}\mathrm{R} \\
\mathrm{i} \\
\mathrm{n}\end{array}$ & freq & $\mathrm{SNF}=0$ & $\mathrm{SNF}=1$ & $\mathrm{SNF}=0$ & $\mathrm{SNF}=1$ & ratio & $\mathrm{p}$ (margin $)$ \\
\hline 1 & 0 & 0 & 0 & 0 & 0 & 0 & 0 & 0 & 0 & 0 & 1501 & 88.61 & 11.39 & 88.44 & 11.56 & 0.66 & 0.00 \\
\hline 2 & 0 & 0 & 0 & 0 & 0 & 0 & 0 & 1 & 0 & 0 & 43 & 74.42 & 25.58 & 68.89 & 31.11 & 1.77 & 0.02 \\
\hline 3 & 0 & 0 & 0 & 0 & 0 & 1 & 0 & 0 & 0 & 0 & 1771 & 84.30 & 15.70 & 84.27 & 15.73 & 0.90 & 0.05 \\
\hline 4 & 0 & 0 & 0 & 0 & 0 & 1 & 0 & 0 & 0 & 1 & 21 & 52.38 & 47.62 & 59.91 & 40.09 & 2.28 & 0.01 \\
\hline 5 & 0 & 0 & 0 & 0 & 0 & 1 & 0 & 0 & 1 & 0 & 51 & 62.75 & 37.26 & 69.83 & 30.17 & 1.72 & 0.02 \\
\hline 6 & 0 & 0 & 0 & 0 & 0 & 1 & 1 & 0 & 0 & 0 & 41 & 60.98 & 39.02 & 60.66 & 39.34 & 2.24 & 0.00 \\
\hline 7 & 0 & 0 & 0 & 0 & 1 & 1 & 0 & 0 & 0 & 0 & 31 & 58.07 & 41.94 & 59.20 & 40.80 & 2.32 & 0.00 \\
\hline 8 & 0 & 1 & 0 & 0 & 0 & 1 & 0 & 0 & 0 & 0 & 41 & 65.85 & 34.15 & 70.14 & 29.86 & 1.70 & 0.04 \\
\hline 9 & 1 & 0 & 0 & 0 & 0 & 0 & 0 & 1 & 0 & 0 & 18 & 50.00 & 50.00 & 59.81 & 40.19 & 2.29 & 0.01 \\
\hline 10 & 1 & 0 & 0 & 0 & 0 & 1 & 0 & 0 & 0 & 0 & 329 & 78.42 & 21.58 & 78.26 & 21.74 & 1.24 & 0.05 \\
\hline 11 & 1 & 0 & 0 & 0 & 0 & 1 & 0 & 0 & 1 & 0 & 16 & 50.00 & 50.00 & 60.87 & 39.13 & 2.23 & 0.03 \\
\hline 12 & 1 & 1 & 0 & 0 & 0 & 1 & 0 & 0 & 0 & 0 & 13 & 84.62 & 15.39 & 61.22 & 38.78 & 2.21 & 0.05 \\
\hline & & & & & & & & & & & 4336 & 82.45 & 17.55 & 82.45 & 17.55 & & \\
\hline
\end{tabular}

These results show that the occurrence of one or more of the Comorbidity IVshypothyroidism (Rhy), anemia (Rau), persistent mental disorders (Rmd), Parkinson's disease (Rpa), unspecified glaucoma (Rug), heart failure (Rhf), chronic kidney disease (Rku), other disorders of bone and cartilage (Rbn), and urinary incontinence (Rin) increases the risk of discharge to a skilled nursing facility (SNF). The model component's individual fit table is provided in Appendix I: Supplementary Tables. Another view of the combinations of these comorbidities is presented in the decision tree in

Figure 10 below. 


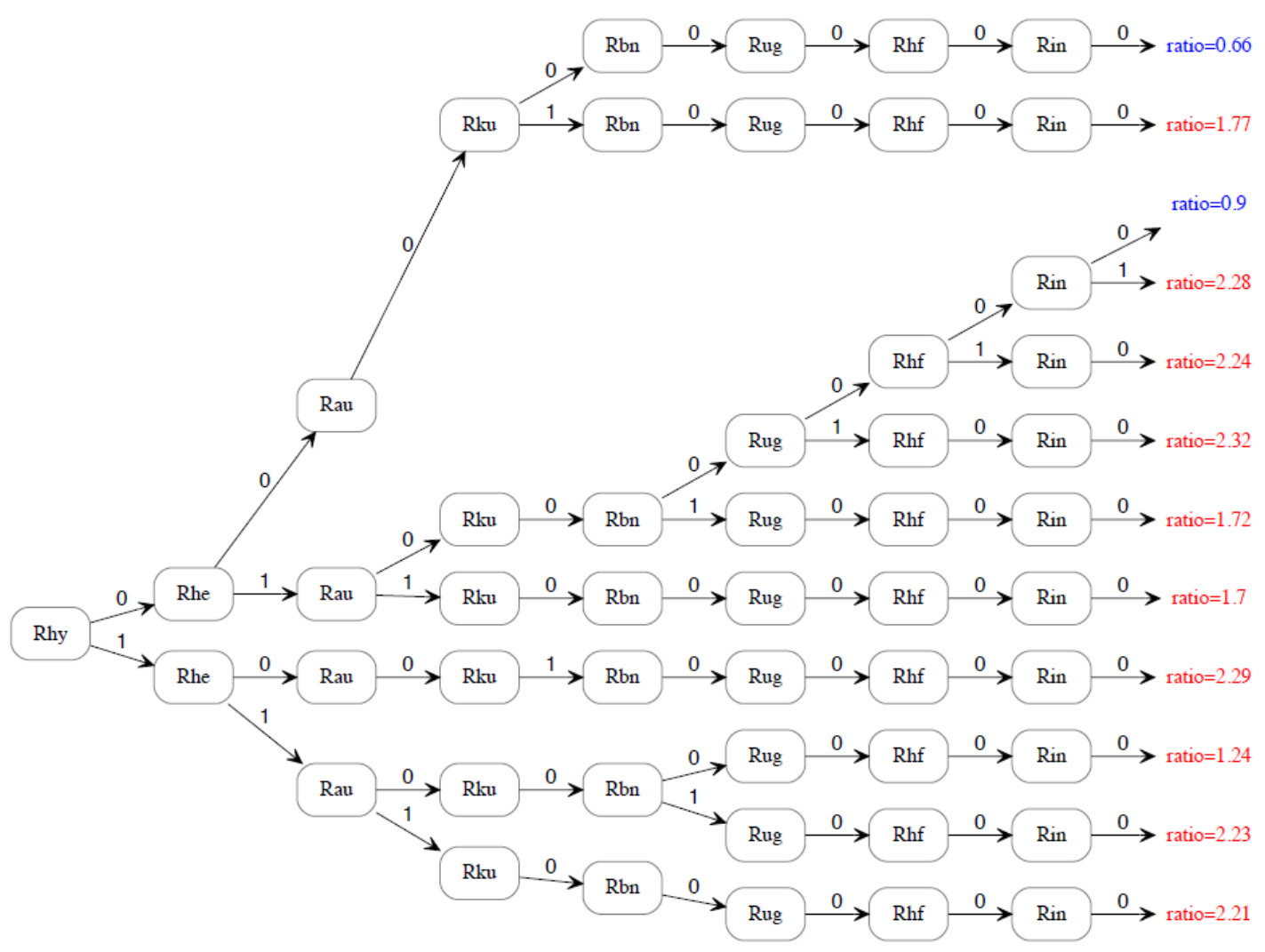

Figure 10. Decision Tree for DV SNF (Knee) with Comorbidity IVs for Best Model Rhy SNF : Rau SNF : Rmd SNF : Rpa SNF : Rug SNF : Rhe SNF : Rhf SNF : Rku SNF : Rbn SNF : Rin SNF.

Hip Analysis

As in the Knee Analysis presented for SNF above, this section analyzes the hip data seen previously in the analysis of DV Cp, but with the DV Skilled Nursing Facility (SNF) instead. SNF is a binary variable, and the positive value marks cases where a patient has been discharged to a skilled nursing facility after a hip replacement. The occurrence of SNF is more common than with the DV Cp, present in approximately 14\% of cases, while DV Cp had positive occurrence of approximately 5\% (Table 37). As before, new searches were conducted with Occam to select best models, considering the 
combined All IVs, and then separately the Comorbidity IVs alone. The set of eight administrative All IVs remains the same for these analyses.

All IVs

\section{- Coarse Searches (Models without Loops)}

The hip data set used for SNF analysis contained 71 IVs, selected through the same variable reduction method described in previous sections. That is, variables found to be individually predictive with $\mathrm{p} \leq 0.05$ were retained, as were a subset of IVs from the literature. The results of searching for predictive models with this data set are summarized in Table 54. As with other analyses presented here, the summary first presents the ten variables that best predict SNF individually. The best of these is financial class $(\mathrm{Fc})$, with a reduction of uncertainty $(\% \Delta \mathrm{H})$ of $12.57 \%$. This IV was also the top single predictor for the Knee Analysis of DV SNF. The other top single predictors are listed as well, sorted by the reduction in uncertainty for each model. The top seven of these predictors are all Hospital IVs, and only two of the ten are Comorbidity IVs. 
Table 54. Summary of Search Results (Hip) for All IVs for SNF. Search covers coarse and fine models.

\begin{tabular}{|c|c|c|c|c|c|}
\hline MODEL & $\Delta \mathrm{df}$ & $\Delta \mathrm{BIC}$ & $\% \Delta \mathrm{H}$ & Alpha & Variable description \\
\hline \multicolumn{6}{|c|}{ COARSE, single predictors (top 10) } \\
\hline Fc SNF & 5 & 289.60 & 12.57 & 0.00 & Financial class \\
\hline Ageb SNF & 2 & 299.44 & 12.02 & 0.00 & Age binned \\
\hline S SNF & 42 & -32.63 & 11.67 & 0.00 & Surgeon \\
\hline Svb SNF & 2 & 181.53 & 7.53 & 0.00 & Surgeon volume binned \\
\hline L SNF & 6 & 86.84 & 5.15 & 0.00 & Location \\
\hline Nrb SNF & 2 & 78.28 & 3.60 & 0.00 & Number of risks binned \\
\hline Ad SNF & 38 & -219.99 & 3.30 & 0.00 & Admit diagnosis \\
\hline Rhy SNF & 1 & 30.41 & 1.47 & 0.00 & Unspecified acquired hypothyroidism (244.9) \\
\hline Da SNF & 5 & -2.44 & 1.44 & 0.00 & Day of admit \\
\hline Rhe SNF & 1 & 25.46 & 1.28 & 0.00 & Unspecified essential hypertension (401.9) \\
\hline MODEL & $\Delta \mathrm{df}$ & $\Delta \mathrm{BIC}$ & $\% \Delta \mathrm{H}$ & Alpha & Variable description \\
\hline \multicolumn{6}{|c|}{ COARSE, IVs in AIC or BIC models but not in top 10} \\
\hline Rnr SNF (rank 15) & 1 & 11.29 & 0.74 & 0.00 & $\begin{array}{l}\text { Aseptic necrosis of head and neck of femur } \\
(733.42)\end{array}$ \\
\hline Rhd SNF (rank 24) & 1 & 4.82 & 0.49 & 0.00 & Acquired hypothyroidism (244) \\
\hline Rml SNF (rank 29) & 1 & 2.84 & 0.42 & 0.00 & Memory loss (780.93) \\
\hline MODEL & $\Delta \mathrm{df}$ & $\triangle \mathrm{BIC}$ & $\% \Delta \mathrm{H}$ & Alpha & Variable description \\
\hline \multicolumn{6}{|c|}{ COARSE, best model (loopless) } \\
\hline \multicolumn{6}{|c|}{$\Delta \mathrm{BIC}$ (best model) } \\
\hline Ageb Svb SNF & 8 & 445.91 & 19.44 & 0.00 & Age binned, Surgeon volume binned \\
\hline \multicolumn{6}{|c|}{ Inc.P \& $\triangle \mathrm{AIC}$ (same best model) } \\
\hline Ageb Svb Rhy Rmo SNF & 35 & 297.19 & 22.08 & 0.00 & $\begin{array}{l}\text { Age binned, Surgeon volume binned, Unspecified } \\
\text { acquired hypothyroidism (244.9), Morbid obesity } \\
\text { (278.01) }\end{array}$ \\
\hline \begin{tabular}{|l|l} 
MODEL & \\
\end{tabular} & $\Delta \mathrm{df}$ & $\Delta \mathrm{BIC}$ & $\% \Delta \mathrm{H}$ & Alpha & Variable description \\
\hline \multicolumn{6}{|c|}{ FINE, best models (with loops) } \\
\hline \multicolumn{6}{|c|}{$\Delta \mathrm{BIC}$ (best model) } \\
\hline $\begin{array}{l}\text { Fc SNF : Ageb SNF : } \\
\text { Svb SNF :Nrb SNF }\end{array}$ & 11 & 531.17 & 23.61 & 0.00 & $\begin{array}{l}\text { Financial class, Age binned, Surgeon volume } \\
\text { binned, Number of risks binned }\end{array}$ \\
\hline \multicolumn{6}{|c|}{ Inc.P \& $\triangle$ AIC (same best model) } \\
\hline $\begin{array}{l}\text { Fc SNF : Ageb SNF : } \\
\text { Svb SNF : Nrb SNF : } \\
\text { Rhd SNF : Rnr SNF : } \\
\text { Rml SNF }\end{array}$ & 14 & 527.73 & 24.41 & 0.01 & $\begin{array}{l}\text { Financial class, Age binned, Surgeon volume } \\
\text { binned, Number of risks binned, Acquired } \\
\text { hypothyroidism (244), Aseptic necrosis of head and } \\
\text { neck of femur (733.42), Memory loss (780.93) }\end{array}$ \\
\hline
\end{tabular}

After the single-predictor models, the next models to consider are those also selected by coarse (or loopless) search, but now also allowing for multiple predicting variables in the single component. (These are given in Table 54, under "COARSE, best model (loopless)"). The best of these models, selected by the $\Delta \mathrm{BIC}$ criterion, is Ageb Svb SNF, where the IVs Age binned (Ageb) and Surgeon volume binned (Svb) predict SNF 
with a of $\% \Delta H$ 19.44. As in other searches, the best model selected does not necessarily reflect the top single-predicting variables, indicating that the included variables (Ageb and $\mathrm{Svb}$ ) together balanced the reduction of uncertainty and increase in complexity (here, a $\Delta \mathrm{df}=8$ ) better than other IV combinations.

- Fine Searches (Models with Loops)

When allowing for multiple predicting components (that is, models with loops), the search for best model selects a model with four components: Fc SNF : Ageb SNF : Svb SNF : Nrb SNF. This model is the best by the BIC criterion for predicting the DV SNF in the Hip data set of All IVs. This model reduces uncertainty by $23.61 \%$ $(\% \Delta \mathrm{H})$, using 11 degrees of freedom $(\Delta \mathrm{df})$. All four of the IVs included in this model are All IVs: Financial class (Fc), Age binned (Ageb), Surgeon volume binned (Svb), Number of risks binned $(\mathrm{Nrb})$. The best model under the less conservative criterion of $\triangle \mathrm{AIC}$ also includes three Comorbidity IVs, and is more complex as a result, with $\Delta \mathrm{df}$ of 14 . In the similar results for Knee analysis of DV SNF, Comorbidity IVs were included in the models selected by each criterion.

\section{- Comorbidity IVs Only}

\section{- Coarse Searches (Models without Loops)}

When considering only the variables in the Comorbidity IVs data set, the most predictive models are substantially different. The results given in Table 54 above include relatively more of the All IVs and few of the Comorbidity IVs, so the list of the ten best single predictors in Table 55 is mostly different by necessity. (The reductions in uncertainty $(\% \Delta \mathrm{H})$ for these predictors are also lower than the best values in Table 54, or 
else they would have been included in that table.) The best single predictor of the

Comorbidity IVs by uncertainty reduction is unspecified acquired hypothyroidism (Rhy),

with a $\% \Delta \mathrm{H}$ of 1.47 .

Table 55. Summary of Search Results (Hip) for Comorbidity IVs for SNF.

Search covers both coarse and fine models.

\begin{tabular}{|c|c|c|c|c|c|}
\hline MODEL & $\Delta \mathrm{df}$ & $\Delta \mathrm{BIC}$ & $\% \Delta \mathrm{H}$ & Alpha & Variable description \\
\hline \multicolumn{6}{|c|}{ COARSE, single predictors } \\
\hline Rhy SNF & 1 & 30.41 & 1.47 & 0.00 & Unspecified acquired hypothyroidism (244.9) \\
\hline Rhe SNF & 1 & 25.46 & 1.28 & 0.00 & Unspecified essential hypertension (401.9) \\
\hline Rav SNF & 1 & 15.99 & 0.92 & 0.00 & Aortic valve disorders (424.1) \\
\hline Rrd SNF & 1 & 15.89 & 0.91 & 0.00 & Unspecified hypertensive renal disease (403.9) \\
\hline Rao SNF & 1 & 15.61 & 0.90 & 0.00 & Chronic airway obstruction (496) \\
\hline Rhf SNF & 1 & 12.87 & 0.80 & 0.00 & Heart failure (428) \\
\hline Rnr SNF & 1 & 11.29 & 0.74 & 0.00 & $\begin{array}{l}\text { Aseptic necrosis of head and neck of femur } \\
(733.42)\end{array}$ \\
\hline Rbn SNF & 1 & 10.25 & 0.70 & 0.00 & Other disorders of bone and cartilage (733) \\
\hline Rhh SNF & 1 & 7.98 & 0.61 & 0.00 & Hyposmolality and/or hyponatremia (276.1) \\
\hline Rkd SNF & 1 & 7.80 & 0.60 & 0.00 & Chronic kidney disease, Stage III (585.3) \\
\hline MODEL & $\Delta \mathrm{df}$ & $\triangle \mathrm{BIC}$ & $\% \Delta \mathrm{H}$ & Alpha & Variable description \\
\hline \multicolumn{6}{|c|}{ COARSE, IVs in AIC or BIC models but not in top 10} \\
\hline Rin SNF (rank 12) & 1 & 6.36 & 0.55 & 0.00 & Urinary incontinence (788.3) \\
\hline Rse SNF (rank 13) & 1 & 6.08 & 0.54 & 0.00 & Senile osteoporosis (733.01) \\
\hline Rug SNF (rank 19) & 1 & 3.21 & 0.43 & 0.00 & Unspecified glaucoma (365.9) \\
\hline Rml SNF (rank 21) & 1 & 2.84 & 0.42 & 0.00 & Memory loss (780.93) \\
\hline MODEL & $\Delta \mathrm{df}$ & $\Delta \mathrm{BIC}$ & $\% \Delta \mathrm{H}$ & Alpha & Variable description \\
\hline \multicolumn{6}{|c|}{ COARSE, best model (loopless) } \\
\hline \multicolumn{6}{|l|}{$\triangle \mathrm{BIC}$ (best model) } \\
\hline Rhy Rhe Rrd SNF & 7 & 46.95 & 3.94 & 0.00 & $\begin{array}{l}\text { Unspecified acquired hypothyroidism (244.9), } \\
\text { Unspecified essential hypertension (401.9), } \\
\text { Unspecified hypertensive renal disease (403.9) }\end{array}$ \\
\hline \multicolumn{6}{|c|}{$\triangle \mathrm{AIC}$ (best model) } \\
\hline Rhy Rhe Rrd Rao SNF & 15 & 7.77 & 4.91 & 0.00 & $\begin{array}{l}\text { Unspecified acquired hypothyroidism (244.9), } \\
\text { Unspecified essential hypertension (401.9), } \\
\text { Unspecified hypertensive renal disease (403.9), } \\
\text { Chronic airway obstruction (496) }\end{array}$ \\
\hline \multicolumn{6}{|c|}{ Inc.P (best model) } \\
\hline $\begin{array}{l}\text { Rhy Rhe Rrd Rbn Rin } \\
\text { SNF }\end{array}$ & 31 & -95.30 & 5.90 & 0.03 & $\begin{array}{l}\text { Unspecified acquired hypothyroidism (244.9), } \\
\text { Unspecified essential hypertension (401.9), } \\
\text { Unspecified hypertensive renal disease (403.9), } \\
\text { Other disorders of bone and cartilage (733), } \\
\text { Urinary incontinence (788.3) }\end{array}$ \\
\hline MODEL & $\Delta \mathrm{df}$ & $\triangle \mathrm{BIC}$ & $\% \Delta \mathrm{H}$ & Alpha & Variable description \\
\hline
\end{tabular}




\begin{tabular}{|c|c|c|c|c|c|}
\hline \multicolumn{6}{|c|}{ FINE, best models (with loops) } \\
\hline \multicolumn{6}{|c|}{$\Delta \mathrm{BIC}$ (best model) } \\
\hline $\begin{array}{l}\text { Rhy SNF : Rhh SNF : } \\
\text { Rug SNF : Rhe SNF : } \\
\text { Rrd SNF : Rav Rbn } \\
\text { SNF : Rao SNF : Rse } \\
\text { SNF : Rnr SNF }\end{array}$ & 11 & 111.97 & 7.65 & 0.00 & $\begin{array}{l}\text { Unspecified acquired hypothyroidism (244.9), } \\
\text { Hyposmolality and/or hyponatremia (276.1), } \\
\text { Unspecified glaucoma (365.9), Unspecified } \\
\text { essential hypertension (401.9), Unspecified } \\
\text { hypertensive renal disease (403.9), Aortic valve } \\
\text { disorders (424.1) + Other disorders of bone and } \\
\text { cartilage (733), Chronic airway obstruction (496), } \\
\text { Senile osteoporosis (733.01), Aseptic necrosis of } \\
\text { head and neck of femur (733.42) }\end{array}$ \\
\hline \multicolumn{6}{|c|}{ Inc.P \& $\triangle \mathrm{AIC}$ (same best model) } \\
\hline $\begin{array}{l}\text { Rhy SNF : Rhh SNF : } \\
\text { Rug SNF : Rhe SNF : } \\
\text { Rrd SNF : Rav Rbn } \\
\text { SNF : Rao SNF : Rse } \\
\text { SNF : Rnr SNF : Rml } \\
\text { SNF : Rin SNF }\end{array}$ & 13 & 110.79 & 8.22 & 0.01 & $\begin{array}{l}\text { Unspecified acquired hypothyroidism (244.9), } \\
\text { Hyposmolality and/or hyponatremia (276.1), } \\
\text { Unspecified glaucoma (365.9), Unspecified } \\
\text { essential hypertension (401.9), Unspecified } \\
\text { hypertensive renal disease (403.9), Aortic valve } \\
\text { disorders (424.1) + Other disorders of bone and } \\
\text { cartilage (733), Chronic airway obstruction (496), } \\
\text { Senile osteoporosis (733.01), Aseptic necrosis of } \\
\text { head and neck of femur (733.42), Memory loss } \\
\text { (780.93), Urinary incontinence (788.3) }\end{array}$ \\
\hline
\end{tabular}

When the coarse search is widened to include more than one predicting variable in its (loopless) models, the best model by $\triangle \mathrm{BIC}$ is Rhy Rhe Rrd SNF. This model combines the IVs unspecified acquired hypothyroidism (Rhy), unspecified essential hypertension (Rhe), and unspecified hypertensive renal disease (Rrd), resulting in a $\% \Delta \mathrm{H}$ of only 3.94. This is much lower than the value for the model above that included All IVs (Ageb Svb SNF), which reduced uncertainty by $19.44 \%$.

\section{- Fine Searches (Models with Loops)}

The final step in the Hip analysis of the Comorbidity IVs data set is to allow for the inclusion of models with loops. Like the search for loopless models with this data set, the results continue to show that the Comorbidity IVs do not predict as well as the All IVs do. The best model by $\Delta$ BIC (Rhy SNF : Rhh SNF : Rug SNF : Rhe SNF : Rrd SNF : Rav Rbn SNF : Rao SNF : Rse SNF : Rnr SNF) includes 10 IVs in nine components, but 
only reduces uncertainty by $7.65 \%$. This model uses 11 degrees of freedom, which is also the $\Delta \mathrm{df}$ of the best model from the All IVs data set, though that model achieved a $\% \Delta \mathrm{H}$ of 23.61 for the same complexity, with four predicting variables.

\section{- Comparing Search Results (Hip, SNF)}

All IVs and the Comorbidity IVs searches yield two sets of results that can be compared to each other and interpreted into a three-tiered classification of predicting variables for DV SNF. These three sets of IVs are shown in Table 56.

Table 56. The 3-Tiered Classification of Predicting Variables for DV SNF, Hip Analysis.

\begin{tabular}{|l|l|}
\hline Tier & Variables \\
\hline Tier 1 - Most Important & Fc, Ageb, Svb, Nrb \\
\hline Tier 2 & Rhy, Rur \\
\hline Tier 3 & Rml, Rhh, Rug, Rhe, Rrd, Rav, Rnn, Rao, Rse \\
\hline
\end{tabular}

The first tier includes the most important predicting variables, which are those that were included in the best model by BIC in the All IVs data set: Fc, Ageb, Svb, and Nrb.

The Tier 2 variables are those that appeared both in the best model by AIC in the combined All IVs search, and in the best model by BIC in the Comorbidity IVs search. This tier adds the two Comorbidity IVs, Rhy and Rur.

Tier 3 includes those variables that occur in either of the two searches from Tier 2 but not both. This tier includes nine Comorbidity IVs Rml, Rhh, Rug, Rhe, Rrd, Rav, Rnn, Rao, and Rse. 
- Model FIT

The best fine-grained model for All IVs, Fc SNF : Ageb SNF : Svb SNF : Nrb SNF, which reduced uncertainty by $23.61 \%$, is explained in detail by the conditional probability distribution in Table 57 below. When Financial class $(\mathrm{Fc})=1$, one tends to get orange, and when $\mathrm{Fc}=2$, one tends to get blue, but there are some exceptions on lines 16, 17, 25, 26 (Table 57). Note that in this composite table one gets only Fc $=1$ or 2; Fc can also be 3, 4, 5, 6, but these other states have much lower frequencies, so one doesn't see them in a composite table. 
Table 57. Fit Table (Hip) for All IVs for Best Model

Fc SNF : Ageb SNF : Svb SNF : Nrb SNF.

Blue rows indicate ratio $<0.90$ and orange rows indicate ratio $>1.10$.

(Ratios between 0.91 and 1.09 are close to the margins and are excluded.)

\begin{tabular}{|c|c|c|c|c|c|c|c|c|c|c|c|}
\hline & IVs & \multicolumn{1}{l|}{ Data } & \multicolumn{2}{l|}{ Model } & & \\
\hline & & & & & & \multicolumn{2}{l|}{ obs. p(DV|IV) } & \multicolumn{2}{c|}{ calc. q(DV|I $)$} & & \\
\hline$\#$ & Fc & Ageb & Svb & Nrb & freq & SNF=0 & SNF=1 & SNF=0 & SNF=1 & Ratio & p(margin) \\
\hline 15 & 1 & 2 & 2 & 3 & 56 & 71.43 & 28.57 & 73.48 & 26.52 & 1.86 & 0.01 \\
\hline 16 & 1 & 2 & 3 & 1 & 43 & 97.67 & 2.33 & 98.27 & 1.73 & 0.12 & 0.02 \\
\hline 17 & 1 & 2 & 3 & 2 & 53 & 100.00 & 0.00 & 97.58 & 2.42 & 0.17 & 0.01 \\
\hline 19 & 1 & 3 & 1 & 1 & 85 & 71.77 & 28.24 & 75.77 & 24.23 & 1.70 & 0.01 \\
\hline 20 & 1 & 3 & 1 & 2 & 128 & 69.53 & 30.47 & 69.00 & 31.00 & 2.17 & 0.00 \\
\hline 21 & 1 & 3 & 1 & 3 & 172 & 60.47 & 39.54 & 55.69 & 44.31 & 3.11 & 0.00 \\
\hline 22 & 1 & 3 & 2 & 1 & 94 & 61.70 & 38.30 & 67.29 & 32.71 & 2.29 & 0.00 \\
\hline 23 & 1 & 3 & 2 & 2 & 114 & 56.14 & 43.86 & 59.41 & 40.59 & 2.85 & 0.00 \\
\hline 24 & 1 & 3 & 2 & 3 & 142 & 47.89 & 52.11 & 45.25 & 54.75 & 3.84 & 0.00 \\
\hline 25 & 1 & 3 & 3 & 1 & 83 & 97.59 & 2.41 & 94.41 & 5.59 & 0.39 & 0.02 \\
\hline 26 & 1 & 3 & 3 & 2 & 110 & 91.82 & 8.18 & 92.32 & 7.68 & 0.54 & 0.05 \\
\hline 28 & 2 & 1 & 1 & 1 & 131 & 99.24 & 0.76 & 97.56 & 2.45 & 0.17 & 0.00 \\
\hline 29 & 2 & 1 & 1 & 2 & 95 & 97.90 & 2.11 & 96.60 & 3.40 & 0.24 & 0.00 \\
\hline 31 & 2 & 1 & 2 & 1 & 127 & 98.43 & 1.58 & 96.33 & 3.67 & 0.26 & 0.00 \\
\hline 32 & 2 & 1 & 2 & 2 & 104 & 97.12 & 2.89 & 94.92 & 5.08 & 0.36 & 0.01 \\
\hline 34 & 2 & 1 & 3 & 1 & 160 & 99.38 & 0.63 & 99.54 & 0.46 & 0.03 & 0.00 \\
\hline 35 & 2 & 1 & 3 & 2 & 116 & 99.14 & 0.86 & 99.35 & 0.65 & 0.05 & 0.00 \\
\hline 36 & 2 & 1 & 3 & 3 & 85 & 98.82 & 1.18 & 98.86 & 1.14 & 0.08 & 0.00 \\
\hline 37 & 2 & 2 & 1 & 1 & 51 & 98.04 & 1.96 & 97.11 & 2.89 & 0.20 & 0.02 \\
\hline 38 & 2 & 2 & 1 & 2 & 46 & 93.48 & 6.52 & 95.99 & 4.01 & 0.28 & 0.05 \\
\hline 40 & 2 & 2 & 2 & 1 & 48 & 93.75 & 6.25 & 95.67 & 4.33 & 0.30 & 0.05 \\
\hline 43 & 2 & 2 & 3 & 1 & 90 & 98.89 & 1.11 & 99.45 & 0.55 & 0.04 & 0.00 \\
\hline 44 & 2 & 2 & 3 & 2 & 91 & 98.90 & 1.10 & 99.23 & 0.77 & 0.05 & 0.00 \\
\hline 45 & 2 & 2 & 3 & 3 & 54 & 98.15 & 1.85 & 98.65 & 1.35 & 0.09 & 0.01 \\
\hline & & & & & 3204 & 85.74 & 14.26 & 85.74 & 14.26 & 1.00 & \\
\hline$\#$ & Fc & Ageb & Svb & Nrb & freq & SNF=0 & SNF=1 & SNF=0 & SNF=1 & Ratio & p(margin) \\
\hline
\end{tabular}

Each of the component's individual projections is somewhat informative, yet not as informative as the full model's joint distribution. These components are provided in the Appendix I: Supplementary Tables, Table 111 through Table 117.

The joint probability distribution below in Table 58 shows the details for the best fine model from the hip Comorbidity IVs search Rhy SNF : Rhh SNF : Rug SNF : Rhe SNF : Rrd SNF : Rav Rbn SNF : Rao SNF : Rse SNF : Rnr SNF. In this distribution, each of the IVs is either 0 (comorbidity absent) or 1 (comorbidity present). 
Row 1 is the case where all of the comorbidities included in the model are absent, which lowers the risk of Skilled Nursing Facility (SNF) from 14.26\% (the model's marginal probability) to $7.85 \%$, with a ratio of 0.55

Table 58. Fit Table (Hip) for Comorbidity IVs for Best Model Rhy SNF : Rhh SNF : Rug SNF : Rhe SNF : Rrd SNF : Rav Rbn SNF : Rao SNF : Rse SNF : Rnr SNF . Blue rows indicate ratio $<0.90$ and orange rows indicate ratio $>1.10$. (Ratios between 0.91 and 1.09 are close to the margins and are excluded.)

\begin{tabular}{|c|c|c|c|c|c|c|c|c|c|c|c|c|c|c|c|c|c|}
\hline \multicolumn{11}{|c|}{ IVs } & \multicolumn{3}{|l|}{ Data } & \multirow{2}{*}{\multicolumn{2}{|c|}{$\begin{array}{l}\text { Model } \\
\text { calc. } \mathrm{g}(\mathrm{DV} \mid \mathrm{IV})\end{array}$}} & \multirow[b]{3}{*}{ ratio } & \multirow[b]{3}{*}{$\mathrm{p}($ margin $)$} \\
\hline & & & & & & & & & & & & obs. p( & DV|IV) & & & & \\
\hline \# & $\overrightarrow{\underline{a}}$ & 류 & $\overline{\underline{\alpha}}$ & $\frac{\square}{\simeq}$ & $\bar{\varepsilon}$ & $\stackrel{\vec{z}}{4}$ & $\stackrel{8}{\approx}$ & 气్๊ & $\stackrel{0}{2}$ & 表 & freq & $\mathrm{SNF}=0$ & $\mathrm{SNF}=1$ & $\mathrm{SNF}=0$ & $\mathrm{SNF}=1$ & & \\
\hline 1 & 0 & 0 & 0 & 0 & 0 & 0 & 0 & 0 & 0 & 0 & 1371 & 92.34 & 7.66 & 92.15 & 7.85 & 0.55 & 0.00 \\
\hline 10 & 0 & 0 & 0 & 0 & 1 & 0 & 0 & 0 & 0 & 0 & 42 & 71.43 & 28.57 & 4.60 & 25.40 & 1.78 & \\
\hline 18 & 0 & 0 & 0 & 1 & 0 & 0 & 0 & 0 & 0 & 1 & & 73.47 & 26.53 & 88.93 & 31.07 & 2.18 & 0.00 \\
\hline 20 & 0 & 0 & 0 & 1 & 0 & 0 & 0 & 1 & 0 & 0 & 4 & 79.1 & 20.93 & 2 & 24. & 1.1 & \\
\hline 22 & 0 & 0 & 0 & 1 & 0 & 0 & 1 & 0 & 0 & 0 & & 76.9 & 23.08 & & 27.99 & 1.96 & 0.05 \\
\hline 26 & 0 & 0 & 0 & 1 & 0 & 1 & 0 & 0 & 0 & $\underline{0}$ & & & 50.00 & & 55. & 3.8 & \\
\hline 50 & 1 & 0 & 0 & 0 & 1 & 0 & 0 & 0 & 0 & 0 & 1 & 78.57 & 21.43 & 59. & 40.67 & 2.85 & \\
\hline 53 & 1 & 0 & 0 & 1 & 0 & 0 & 0 & 0 & 0 & 0 & 17 & 74 & 25.15 & 44 & 24. & 1.69 & 00 \\
\hline 56 & 1 & 0 & 0 & 1 & 0 & 0 & 0 & 1 & 0 & 0 & 1 & 66. & 33.33 & 59. & 40.13 & 2.81 & 0.01 \\
\hline & & & & & & & & & & & 3204 & 85.74 & 14.26 & 85.74 & 14.26 & & \\
\hline
\end{tabular}

The additional IV states show if one or more of the comorbidities from the model are present, the risk is increased. This is illustrated in the decision tree below in Figure 11. 


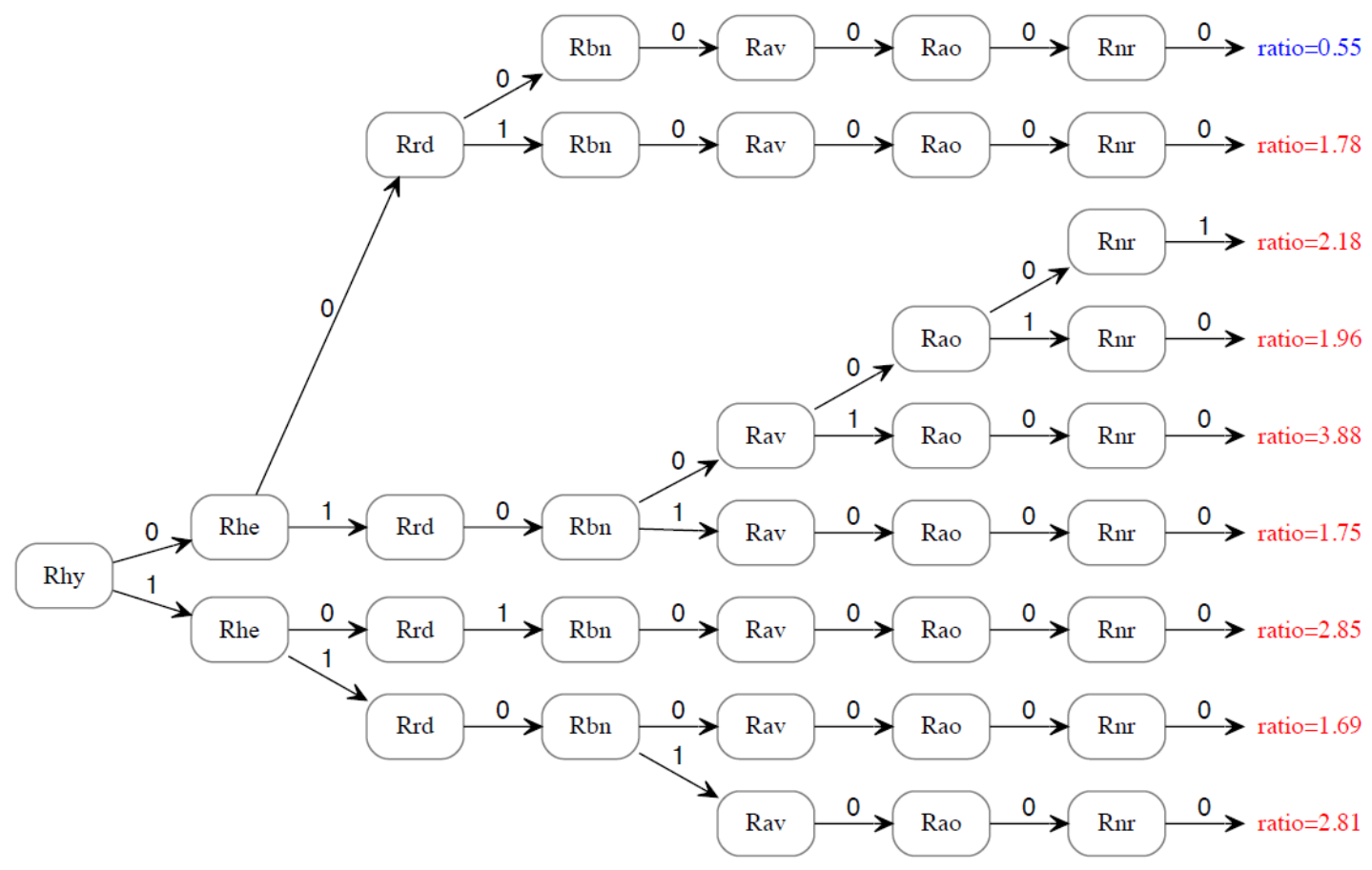

Figure 11. Decision Tree for DV SNF (Hip) with Comorbidity IVs, for Best Model, Rhy SNF : Rhh SNF : Rug SNF :Rhe SNF : Rrd SNF : Rav Rbn SNF : Rao SNF : Rse SNF : Rnr SNF.

Hip \& Knee Summary of Results for SNF

The most important IVs are summarized in Table 59. The IVs that are important across both hip and knee searches are number of risks (Nrb), age (Ageb), surgeon volume (Svb), financial class (Fc), glaucoma (Rug), essential hypertension (Rhe), and acquired hypothyroidism (Rhy)

Table 59. Summary of Most Important IVs by Tier across Hip and Knee for SNF.

\begin{tabular}{|r|l|l|}
\hline Tier & Knee & Hip \\
\hline 1 & L, Fc, Ageb, Svb, Nrb, Rmd, Rug, Rhf & Fc, Ageb, Svb, Nrb \\
\hline 2 & Rpa & Rhy, Rur \\
\hline 3 & Rbp, Rhy, Rau, Rhe, Rku, Rbn, Rin & Rml, Rhh, Rug, Rhe, Rrd, Rav, Rnn, Rao, Rse \\
\hline
\end{tabular}




\section{DV: Readmission $(\mathrm{Re})$}

Knee Analysis

In this results section, another series of best models are proposed and analyzed in detail. This section looks both at (a) All IVs together, and (b) only the Comorbidity IVs.

- All IVs

○ Coarse Searches (Models without Loops)

The original 188 patient Comorbidity IVs were reduced to 35 IVs, of which 17 had $\mathrm{p} \leq 0.05$ in a single predicting search for the dependent variable Readmission (Re), 18 were literature-based but not individually predictive in the search, and five were found both in the literature and in the single predicting search. The search results for the top 10 models are listed in Table 60. 
Table 60. Summary of Search Results (Knee) for All IVs for Readmission (Re). Search covers coarse and fine models.

\begin{tabular}{|c|c|c|c|c|c|}
\hline MODEL & $\Delta \mathrm{df}$ & $\triangle \mathrm{BIC}$ & $\% \Delta \mathrm{H}$ & Alpha & Variable description \\
\hline \multicolumn{6}{|c|}{ COARSE, single predictors (top 10$)$} \\
\hline $\mathrm{Fc} \mathrm{Re}$ & 5 & -28.66 & 1.26 & 0.02 & Financial class \\
\hline Nrb Re & 2 & -4.02 & 1.22 & 0.00 & Number of risks binned \\
\hline $\mathrm{L} \operatorname{Re}$ & 6 & -37.85 & 1.18 & 0.05 & Location \\
\hline $\operatorname{Rco} \operatorname{Re}$ & 1 & 3.88 & 1.17 & 0.00 & Chronic obstructive asthma (493.2) \\
\hline $\operatorname{Rgp} \operatorname{Re}$ & 1 & 1.73 & 0.96 & 0.00 & Repair of cystocele with graft or prosthesis (70.54) \\
\hline Rcj Re & 1 & 0.66 & 0.86 & 0.00 & Contracture of joint, lower leg (718.46) \\
\hline Rci Re & 1 & -0.43 & 0.76 & 0.00 & Other forms of chronic ischemic heart disease (414) \\
\hline Rhf Re & 1 & -1.58 & 0.65 & 0.01 & Heart failure (428) \\
\hline Rca Re & 1 & -1.83 & 0.63 & 0.01 & $\begin{array}{l}\text { Coronary atherosclerosis of native coronary artery } \\
(414.01)\end{array}$ \\
\hline Rdf $\mathrm{Re}$ & 1 & -1.87 & 0.62 & 0.01 & Chronic diastolic heart failure (428.32) \\
\hline MODEL & $\Delta \mathrm{df}$ & $\Delta \mathrm{BIC}$ & $\% \Delta \mathrm{H}$ & Alpha & Variable description \\
\hline \multicolumn{6}{|c|}{ COARSE, IVs in AIC or BIC models but not in top 10} \\
\hline $\operatorname{Rep} \operatorname{Re}(\operatorname{Rank} 12)$ & 1 & -2.54 & 0.56 & 0.02 & Epilepsy (345.9) \\
\hline MODEL & $\Delta \mathrm{df}$ & $\Delta \mathrm{BIC}$ & $\% \Delta \mathrm{H}$ & Alpha & Variable description \\
\hline \multicolumn{6}{|c|}{ COARSE, best model (loopless) } \\
\hline \multicolumn{6}{|c|}{$\Delta \mathrm{BIC}($ best model) } \\
\hline Rco Re & 1 & 3.88 & 1.17 & 0.00 & Chronic obstructive asthma (493.2) \\
\hline \multicolumn{6}{|c|}{ Inc.P \& $\triangle \mathrm{AIC}$ (same best model) } \\
\hline Rco Rcj Rin Re & 7 & -23.56 & 3.35 & 0.01 & $\begin{array}{l}\text { Chronic obstructive asthma (493.2) + Contracture of } \\
\text { joint, lower leg (718.46) + Urinary incontinence }(788.3)\end{array}$ \\
\hline \multicolumn{6}{|c|}{ FINE, best models (with loops) } \\
\hline \multicolumn{6}{|c|}{$\Delta \mathrm{BIC}$ (best model) } \\
\hline $\begin{array}{l}\operatorname{Rci} \operatorname{Re}: \operatorname{Rco} \operatorname{Re}: \\
\operatorname{Rgp} \operatorname{Re}: \operatorname{Rcj} \operatorname{Re}\end{array}$ & 4 & 6.00 & 3.77 & 0.00 & $\begin{array}{l}\text { Other forms of chronic ischemic heart disease (414), } \\
\text { Chronic obstructive asthma (493.2), Repair of cystocele } \\
\text { with graft or prosthesis }(70.54) \text {, Contracture of joint, } \\
\text { lower leg (718.46) }\end{array}$ \\
\hline \multicolumn{6}{|c|}{ Inc.P \& $\triangle \mathrm{AIC}$ (same best model) } \\
\hline $\begin{array}{l}\operatorname{Rep} \operatorname{Re}: \operatorname{Rci} \operatorname{Re}: \\
\operatorname{Rca} \operatorname{Re}: \operatorname{Rdf} \operatorname{Re}: \\
\operatorname{Rco} \operatorname{Re}: \operatorname{Rgp} \operatorname{Re}: \\
\operatorname{Rcj} \operatorname{Re}\end{array}$ & 7 & 42.78 & 5.42 & 0.02 & $\begin{array}{l}\text { Epilepsy (345.9), Other forms of chronic ischemic heart } \\
\text { disease (414), Coronary atherosclerosis of native } \\
\text { coronary artery (414.01), Chronic diastolic heart failure } \\
\text { (428.32), Chronic obstructive asthma (493.2), Repair of } \\
\text { cystocele with graft or prosthesis ( } 70.54) \text {, Contracture of } \\
\text { joint, lower leg (718.46) }\end{array}$ \\
\hline
\end{tabular}

Knowing the financial class $(\mathrm{Fc})$ of the patient is the most predictive single variable, reducing uncertainty $(\% \Delta \mathrm{H})$ by $1.26 \%$. Number of risks $(\mathrm{Nrb})$ followed with a $\% \Delta \mathrm{H}$ of 1.22 and then location $(\mathrm{L})$ at a $\% \Delta \mathrm{H}$ of 1.18 (Table 60 ). The Comorbidity IV that is individually the post predictive of Readmission ( $\mathbf{R e})$ is chronic obstructive asthma (Rco) with a $\% \Delta \mathrm{H}$ of 1.17 , with additional comorbidities listed in Table 60 . The 
best coarse model by $\triangle \mathrm{BIC}$ is the top Comorbidity $I V$ single predictor, Rco $\mathbf{R e}$ with a $\% \Delta H$ of 1.17 .

○ Fine Searches (Models with Loops)

In this next search, models with loops are considered. The best fine model by $\Delta \mathrm{BIC}$ is Rci $\operatorname{Re}: \operatorname{Rco} \boldsymbol{R e}: \operatorname{Rgp} \operatorname{Re}: \operatorname{Rcj} \operatorname{Re}$ with a $\% \Delta \mathrm{H}$ of 3.77 and $\Delta \mathrm{df}=4$ (Table 60 ). In this model, chronic ischemic heart disease (Rci), chronic obstructive asthma (Rco), repair of cystocele with graft or prosthesis (Rgp), and contracture of joint, lower leg (Rcj) provide the most information balanced by their complexity $(\Delta \mathrm{df})$. While this project uses the $\triangle \mathrm{BIC}$ criterion for model selection, it is interesting to see that even with the AIC criterion, the best model selected contains only Comorbidity IVs, and not the top three single predicting variables, which were the All IVs financial class $(\mathrm{Fc})$, number of risks (Nrb), and location (L).

\section{- Comorbidity IVs}

\section{- Coarse Searches (Models without Loops)}

In the next search, only Comorbidity IVs are considered. These search results exclude the three All IVs from the previous search, and therefore an additional three Comorbidity IVs are included in the top 10 single predictors in Table 61. The best models by BIC are the exact same models selected from the prior search. In other words, the best coarse model is Rco $\operatorname{Re}$ with a $\% \Delta \mathrm{H}$ of 1.17 . 
Table 61. Summary of Search Results (Knee) for Comorbidity IVs for DV Readmission (Re). Search covers coarse and fine models.

\begin{tabular}{|c|c|c|c|c|c|}
\hline MODEL & $\Delta \mathrm{df}$ & $\triangle \mathrm{BIC}$ & $\% \Delta \mathrm{H}$ & Alpha & Variable description \\
\hline \multicolumn{6}{|c|}{ COARSE, single predictors } \\
\hline $\operatorname{Rco} \operatorname{Re}$ & 1 & 3.88 & 1.17 & 0.00 & Chronic obstructive asthma (493.2) \\
\hline $\operatorname{Rgp} \operatorname{Re}$ & 1 & 1.73 & 0.96 & 0.00 & Repair of cystocele with graft or prosthesis (70.54) \\
\hline $\operatorname{Rcj} \operatorname{Re}$ & 1 & 0.66 & 0.86 & 0.00 & Contracture of joint, lower leg (718.46) \\
\hline Rci Re & 1 & -0.43 & 0.76 & 0.00 & Other forms of chronic ischemic heart disease (414) \\
\hline Rhf Re & 1 & -1.58 & 0.65 & 0.01 & Heart failure (428) \\
\hline Rca Re & 1 & -1.83 & 0.63 & 0.01 & $\begin{array}{l}\text { Coronary atherosclerosis of native coronary artery } \\
(414.01)\end{array}$ \\
\hline Rdf Re & 1 & -1.87 & 0.62 & 0.01 & Chronic diastolic heart failure (428.32) \\
\hline Rse Re & 1 & -2.44 & 0.57 & 0.01 & Senile osteoporosis (733.01) \\
\hline $\operatorname{Rep} \operatorname{Re}$ & 1 & -2.54 & 0.56 & 0.02 & Epilepsy (345.9) \\
\hline Ros Re & 1 & -2.92 & 0.52 & 0.02 & Obstructive sleep apnea (327.23) \\
\hline MODEL & $\Delta \mathrm{df}$ & $\Delta \mathrm{BIC}$ & $\% \Delta \mathrm{H}$ & Alpha & Variable description \\
\hline \multicolumn{6}{|c|}{ COARSE, best model (loopless) } \\
\hline \multicolumn{6}{|c|}{$\Delta \mathrm{BIC}$ (best model) } \\
\hline Rco Re & 1 & 3.88 & 1.17 & 0.00 & Chronic obstructive asthma (493.2) \\
\hline \multicolumn{6}{|c|}{ Inc.P \& $\triangle \mathrm{AIC}$ (same best model) } \\
\hline Rco Rcj Rin Re & 7 & -23.56 & 3.35 & 0.01 & $\begin{array}{l}\text { Chronic obstructive asthma (493.2) + Contracture of } \\
\text { joint, lower leg }(718.46)+\text { Urinary incontinence }(788.3)\end{array}$ \\
\hline MODEL & $\Delta \mathrm{df}$ & $\Delta \mathrm{BIC}$ & $\% \Delta H$ & Alpha & Variable description \\
\hline \multicolumn{6}{|c|}{ FINE, best models (with loops) } \\
\hline \multicolumn{6}{|c|}{$\triangle \mathrm{BIC}$ (best model) } \\
\hline $\begin{array}{l}\text { Rci Re : Rco Re : } \\
\operatorname{Rgp} \operatorname{Re}: \operatorname{Rcj} \operatorname{Re}\end{array}$ & 4 & 6.00 & 3.77 & 0.00 & $\begin{array}{l}\text { Other forms of chronic ischemic heart disease (414), } \\
\text { Chronic obstructive asthma (493.2), Repair of cystocele } \\
\text { with graft or prosthesis (70.54), Contracture of joint, } \\
\text { lower leg (718.46) }\end{array}$ \\
\hline \multicolumn{6}{|c|}{ Inc.P \& $\triangle \mathrm{AIC}$ (same best model) } \\
\hline $\begin{array}{l}\text { Rep Re : Rci Re : } \\
\text { Rca Re }: \text { Rdf Re : } \\
\operatorname{Rco} \operatorname{Re}: \operatorname{Rgp} \operatorname{Re}: \\
\operatorname{Rcj} \operatorname{Re}\end{array}$ & 7 & -1.84 & 5.42 & 0.02 & $\begin{array}{l}\text { Epilepsy (345.9), Other forms of chronic ischemic heart } \\
\text { disease (414), Coronary atherosclerosis of native } \\
\text { coronary artery (414.01), Chronic diastolic heart failure } \\
\text { (428.32), Chronic obstructive asthma (493.2), Repair of } \\
\text { cystocele with graft or prosthesis (70.54), Contracture of } \\
\text { joint, lower leg ( } 718.46)\end{array}$ \\
\hline
\end{tabular}

\section{- Fine Searches (Models with Loops)}

The Comorbidity IVs search provided no additional best model beyond what was previously identified in the Hospital-Based + Comorbidities search. The best fine-grained BIC model is again Rci $\operatorname{Re}: \operatorname{Rco} \mathbf{R e}: \operatorname{Rgp} \mathbf{R e}: \operatorname{Rcj} \mathbf{R e}$ with a \% $\%$ H of 3.77 (Table 61). 


\section{- Comparing Search Results (Knee, Re)}

All IVs and the Comorbidity IVs searches yield two sets of results that can be compared to each other and interpreted into a three-tiered classification of results, as described below and summarized in Table 62.

Table 62. The 3-Tiered Classification of Predicting Variables for DV: $R e$, Knee Analysis.

\begin{tabular}{|l|l|}
\hline Tier & Variables \\
\hline Tier 1 - Most Important & Rci, Rco, Rgp, Rcj \\
\hline Tier 2 & None \\
\hline Tier 3 & Rep, Rca, Rdf \\
\hline
\end{tabular}

Tier 1 contains variables from the best-by-BIC model from the dataset All IVs. This is the most complete search, and the one that provides the most conservative predictors. The selected variables Rci, Rco, Rgp, and Rcj are shown in the first row of Table 62, and are considered the most important predicting variables.

Tier 2 contains variables not in Tier1, but found in the AIC (less conservative) model of All IVs AND in the BIC model of Comorbidity IVs. For the Knee Analysis of DV Readmission, there are no additional predictors. These are shown in the second row of Table 62.

Finally, this classification places into Tier 3 any variables present elsewhere in the best model search results, but not included in Tier 1 or Tier 2 ; that is, any variables unique to one of the two searches - variables in the AIC model of All IVs but not in the BIC model of Comorbidity IVs, and variables in the BIC model of Comorbidity IVs but not in the AIC model from All IVs. The next most important predicting variables are Rep, Rca, and Rdf as summarized in row 3 of Table 62. 
- Model FIT

The best models for both All IVs search and the Comorbidity IVs search yielded the same best model of Rci Re $: \operatorname{Rco} \mathbf{R e}: \operatorname{Rgp} \operatorname{Re}: \operatorname{Rcj} \mathbf{R e}$. The detailed content of this model is shown below in the joint conditional probability distribution of the model (Table 63). Rows with a frequency $<10$ and $p>0.05$ are excluded.

Table 63. Full Fit Table (Knee) for both All IVs search and Comorbidity IVs search for Best Model: Rci Re : Rco Re : Rgp Re : Rcj Re.

Blue rows indicate ratio $<0.90$ and orange rows indicate ratio $>1.10$. (Ratios between 0.91 and 1.09 are close to the margins and are indicated in gray.)

\begin{tabular}{|c|c|c|c|c|c|c|c|c|c|c|c|}
\hline & \multicolumn{4}{|l|}{ IVs } & \multicolumn{2}{l|}{ Data } & \multicolumn{2}{l|}{ Model } & & \\
\hline & & & & & & \multicolumn{2}{|c|}{ obs. p(DV|IV) } & \multicolumn{2}{c|}{ calc. q(DV|IV) } & & \\
\hline$\#$ & Rci & Rco & Rgp & Rcj & freq & Re $=0$ & $\mathrm{Re}=1$ & $\mathrm{Re}=0$ & $\mathrm{Re}=1$ & ratio & $\mathrm{p}$ (margin) \\
\hline 2 & 0 & 0 & 0 & 1 & 22 & 86.36 & 13.64 & 84.44 & 15.56 & 5.97 & 0.00 \\
\hline 4 & 0 & 1 & 0 & 0 & 38 & 86.84 & 13.16 & 85.73 & 14.27 & 5.48 & 0.00 \\
\hline 6 & 1 & 0 & 0 & 0 & 141 & 93.62 & 6.38 & 93.29 & 6.71 & 2.57 & 0.00 \\
\hline & & & & & 4334 & 97.39 & 2.61 & 97.39 & 2.61 & 1.00 & \\
\hline
\end{tabular}

The presence of contracture of joint, lower leg (Rcj) increases the chance of readmission (Re) from $2.61 \%$ to $15.56 \%$, with a risk ratio of 5.97 (Table 63). Chronic obstructive asthma (Rco) increases readmission ( $\mathrm{Re}$ ) to $14.27 \%$, with a ratio of 5.48, and chronic ischemic heart disease (Rci) increases risk of readmission to $6.71 \%$, with a ratio of 2.57. This is shown clearly in the decision tree below in Figure 12. 


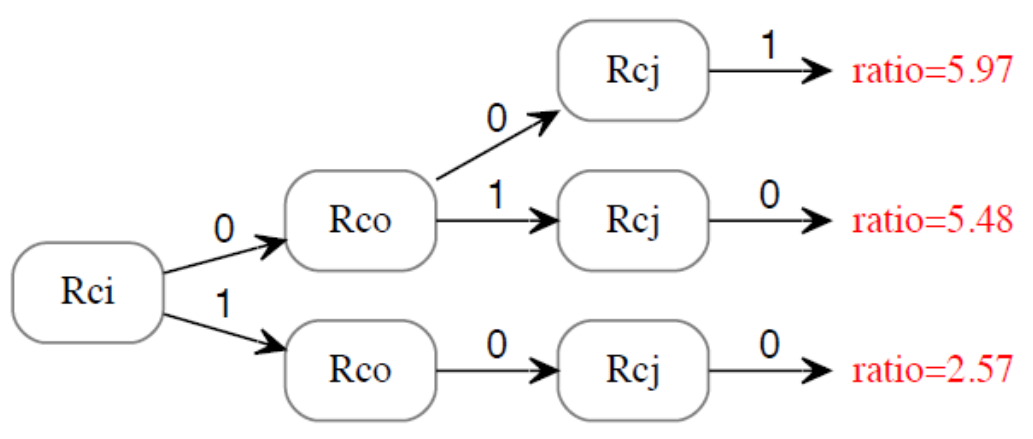

Figure 12. Decision Tree for DV Readmit (Knee) for Best Model Rci Re : Rco Re : Rgp Re : Rcj Re.

Hip Analysis

In this portion of the results section, a similar series of results to the knee analysis are presented for the dependent variable Readmission (Re). Here, two sets of results are again produced: (a) All IVs, and (b) Comorbidity IVs. The hip data set contains the same eight administrative All IVs but a different set of Comorbidity IVs.

- All IVs

- Coarse Searches (Models without Loops)

A single predicting search reduced the IVs in the Hip data set to 30, of which 14 were found to be predictive with $\mathrm{p} \leq 0.05,16$ were retained as literature-based IVs, and only one IV in common.

The top 10 individual predicting variables are listed below in Table 64. The most predictive IV is surgeon (S) with a $\% \Delta \mathrm{H}$ of 8.41 and a $\Delta \mathrm{df}$ of 42 . The day of admit (Da) interestingly is the next single predictor with $\% \Delta \mathrm{H}$ of 2.03 and a $\Delta \mathrm{df}$ of 5 . The best 
loopless coarse model by the $\triangle \mathrm{BIC}$ criterion is the independence model, meaning that nothing predicts Readmission ( $\mathbf{R e})$.

Table 64. Summary of Search Results (Hip) for All IVs for DV Readmit (Re). Search covers directed coarse, and fine models.

\begin{tabular}{|c|c|c|c|c|c|}
\hline MODEL & $\Delta \mathrm{df}$ & $\triangle \mathrm{BIC}$ & $\% \Delta \mathrm{H}$ & Alpha & Variable description \\
\hline \multicolumn{6}{|c|}{ COARSE, single predictors (top 10) } \\
\hline $\mathrm{S} \operatorname{Re}$ & 42 & -271.92 & 8.41 & 0.01 & Surgeon \\
\hline $\mathrm{Da} \mathrm{Re}$ & 5 & -24.18 & 2.03 & 0.01 & Day of admit \\
\hline Nrb Re & 2 & -3.19 & 1.62 & 0.00 & Number of risk binned \\
\hline Ageb Re & 2 & -5.14 & 1.38 & 0.00 & Age binned \\
\hline Svb Re & 2 & -7.91 & 1.03 & 0.02 & Surgeon volume binned \\
\hline Rdd $\operatorname{Re}$ & 1 & -0.29 & 0.97 & 0.01 & Dysthymic disorder (300.4) \\
\hline Rer Re & 1 & -0.95 & 0.89 & 0.01 & Esophageal reflux (530.81) \\
\hline Rki Re & 1 & -1.03 & 0.88 & 0.01 & Chronic kidney disease, Stage II (585.2) \\
\hline Rhp Re & 1 & -1.70 & 0.80 & 0.01 & Hypopotassemia (276.8) \\
\hline Rys Re & 1 & -1.70 & 0.80 & 0.01 & Cardiac dysrhythmias (427) \\
\hline MODEL & $\Delta \mathrm{df}$ & $\Delta \mathrm{BIC}$ & $\% \Delta \mathrm{H}$ & Alpha & Variable description \\
\hline \multicolumn{6}{|c|}{ COARSE, IVs in AIC or BIC models but not in top 10} \\
\hline $\operatorname{Rep}(\operatorname{rank} 11)$ & 1 & -2.04 & 0.75 & 0.01 & Epilepsy (345.9) \\
\hline Rpa (rank 12) & 1 & -2.75 & 0.67 & 0.02 & Parkinson's disease (332) \\
\hline Rhe (rank 13) & 1 & -3.11 & 0.62 & 0.03 & Essential hypertension (401.9) \\
\hline MODEL & $\Delta \mathrm{df}$ & $\triangle \mathrm{BIC}$ & $\% \Delta \mathrm{H}$ & Alpha & Variable description \\
\hline \multicolumn{6}{|c|}{ COARSE, best model (loopless) } \\
\hline \multicolumn{6}{|c|}{$\Delta \mathrm{BIC}$ (best model) } \\
\hline $\operatorname{Re}$ & 0 & 0.00 & 0.00 & 0.00 & * Independence model is the best model \\
\hline \multicolumn{6}{|c|}{$\Delta$ AIC (best model) } \\
\hline Rep Rer Re & 3 & -7.53 & 2.09 & 0.00 & Epilepsy (345.9) + Esophageal reflux (530.81) \\
\hline \multicolumn{6}{|c|}{ Inc.P (best model) } \\
\hline $\mathrm{SRe}$ & 42 & -272.01 & 8.38 & 0.01 & Surgeon \\
\hline MODEL & $\Delta \mathrm{df}$ & $\Delta \mathrm{BIC}$ & $\% \Delta \mathrm{H}$ & Alpha & Variable description \\
\hline \multicolumn{6}{|c|}{ FINE, best models (with loops) } \\
\hline \multicolumn{6}{|c|}{$\Delta \mathrm{BIC}$ (best model) } \\
\hline $\mathrm{Re}$ & 0 & 0.00 & 0.00 & 0.00 & * Independence model is the best model \\
\hline \multicolumn{6}{|c|}{$\triangle \mathrm{AIC}$ (best model) } \\
\hline $\begin{array}{l}\text { Rhp Re : Rdd } \\
\operatorname{Re}: \operatorname{Rpa} \operatorname{Re}: \\
\operatorname{Rep} \operatorname{Re}: \text { Rhe } \\
\operatorname{Re}: \text { Rys Re : } \\
\text { Rki Re }\end{array}$ & 7 & -12.33 & 5.53 & 0.04 & $\begin{array}{l}\text { Hypopotassemia (276.8), Dysthymic disorder (300.4), } \\
\text { Parkinson's disease (332), Epilepsy (345.9), Essential } \\
\text { hypertension (401.9), Cardiac dysrhythmias (427), } \\
\text { Chronic kidney disease, Stage II (585.2) }\end{array}$ \\
\hline \multicolumn{6}{|c|}{ Inc.P (best model) } \\
\hline $\mathrm{S} \mathrm{Re}$ & 42 & -272.01 & 8.38 & 0.01 & Surgeon \\
\hline
\end{tabular}


○ Fine Searches (Models with Loops)

The best fine-grained search allowing for multiple components and models with loops by BIC is simply shown as Re, saying again that nothing predicts better than the independence model (Table 64). In the hip data, the occurrence of readmission was comparable to the knee data readmission rate at $2.72 \%$. For purposes of the readmission analysis, the AIC model will be used. The best fine-grained model by AIC, Rhp Re :

Rdd $\mathbf{R e}: \operatorname{Rpa} \mathbf{R e}: \operatorname{Rep} \mathbf{R e}: \operatorname{Rhe} \mathbf{R e}: \operatorname{Rys} \mathbf{R e}:$ Rki $\mathbf{R e}$, is less conservative but suggests that hypopotassemia (Rhp), dysthymic disorder (Rdd), Parkinson's disease (Rpa), epilepsy (Rep), essential hypertension (Rhe), cardiac dysrhythmias (Rys), and chronic kidney disease (Rki) reduce the uncertainty of readmission with $\% \Delta \mathrm{H}$ of 5.53 and $\Delta \mathrm{df}=7$

- Comorbidity IVs

○ Coarse Searches (Models without Loops)

The top 10 variables from a search only considering the Comorbidity IVs are shown in Table 65. The top single predicting comorbidity for Readmission (Re) is dysthymic disorder (Rdd) followed by additional comorbidities listed in the table below. 
Table 65. Summary of Search Results (Hip) for Comorbidity IVs. Search covers directed coarse, and fine models.

\begin{tabular}{|c|c|c|c|c|c|}
\hline MODEL & $\Delta \mathrm{df}$ & $\Delta \mathrm{BIC}$ & $\% \Delta \mathrm{H}$ & Alpha & Variable description \\
\hline \multicolumn{6}{|c|}{ COARSE, single predictors } \\
\hline Rdd Re & 1 & -0.29 & 0.97 & 0.01 & Dysthymic disorder (300.4) \\
\hline Rer Re & 1 & -0.95 & 0.89 & 0.01 & Esophageal reflux (530.81) \\
\hline Rki Re & 1 & -1.03 & 0.88 & 0.01 & Chronic kidney disease, Stage II (585.2) \\
\hline $\operatorname{Rhp} \operatorname{Re}$ & 1 & -1.71 & 0.80 & 0.01 & Hypopotassemia (276.8) \\
\hline Rys Re & 1 & -1.71 & 0.80 & 0.01 & Cardiac dysrhythmias (427) \\
\hline $\operatorname{Rep} \operatorname{Re}$ & 1 & -2.06 & 0.75 & 0.01 & Epilepsy (345.9) \\
\hline Rpa Re & 1 & -2.76 & 0.66 & 0.02 & Parkinson's disease (332) \\
\hline Rhe Re & 1 & -3.09 & 0.62 & 0.03 & Essential hypertension (401.9) \\
\hline $\operatorname{Rcm} \operatorname{Re}$ & 1 & -4.17 & 0.49 & 0.05 & Other primary cardiomyopathies (425.4) \\
\hline Rvi Re & 1 & -4.44 & 0.45 & 0.06 & Venous (peripheral) insufficiency (459.81) \\
\hline MODEL & $\Delta \mathrm{df}$ & $\Delta \mathrm{BIC}$ & $\% \Delta \mathrm{H}$ & Alpha & Variable description \\
\hline \multicolumn{6}{|c|}{ COARSE, best model (loopless) } \\
\hline \multicolumn{6}{|c|}{$\Delta \mathrm{BIC}$ (best model) } \\
\hline $\operatorname{Re}$ & 0 & 0.00 & 0.00 & 0.00 & * Independence model is the best model \\
\hline \multicolumn{6}{|l|}{$\Delta$ AIC (best model) } \\
\hline Rep Rca Rer Re & 7 & -27.76 & 3.60 & 0.02 & $\begin{array}{l}\text { Epilepsy }(345.9)+\text { Coronary atherosclerosis of } \\
\text { native coronary artery }(414.01)+\text { Esophageal } \\
\text { reflux }(530.81)\end{array}$ \\
\hline \multicolumn{6}{|l|}{ Inc.P (best model) } \\
\hline Rpa Rep Rca Rer Re & 15 & -78.88 & 5.28 & 0.04 & $\begin{array}{l}\text { Parkinson's disease (332) + Epilepsy }(345.9)+ \\
\text { Coronary atherosclerosis of native coronary artery } \\
(414.01)+\text { Esophageal reflux }(530.81)\end{array}$ \\
\hline MODEL & $\Delta \mathrm{df}$ & $\Delta \mathrm{BIC}$ & $\% \Delta \mathrm{H}$ & Alpha & Variable description \\
\hline \multicolumn{6}{|c|}{ FINE, best models (with loops) } \\
\hline \multicolumn{6}{|c|}{$\Delta \mathrm{BIC}$ (best model) } \\
\hline $\operatorname{Re}$ & 0 & 0.00 & 0.00 & 0.00 & * Independence model is the best model \\
\hline \multicolumn{6}{|c|}{ Inc.P \& $\Delta$ AIC (same best model) } \\
\hline $\begin{array}{l}\text { Rdd Re : Rpa Re : } \\
\text { Rep Re : Rhe Re : } \\
\text { Rys Re : Rer Re : } \\
\text { Rki Re }\end{array}$ & 7 & -12.40 & 5.52 & 0.03 & $\begin{array}{l}\text { Dysthymic disorder (300.4), Parkinson's disease } \\
\text { (332), Epilepsy (345.9), Essential hypertension } \\
\text { (401.9), Cardiac dysrhythmias (427), Esophageal } \\
\text { reflux (530.81), Chronic kidney disease, Stage II } \\
(585.2)\end{array}$ \\
\hline
\end{tabular}

\section{- Fine Searches (Models with Loops)}

In the search that allows for loops, and as was seen with the prior search results, the best model by BIC is the independence model, and therefore the best model by AIC will be used for additional analysis. The AIC model is slightly different: Rdd $\mathbf{R e}$ :

Rpa $\operatorname{Re}: \operatorname{Rep} \operatorname{Re}: \operatorname{Rhe} \boldsymbol{\operatorname { R e }}: \operatorname{Rys} \boldsymbol{\operatorname { R e }}: \operatorname{Rer} \boldsymbol{\operatorname { R e }}: \operatorname{Rki} \boldsymbol{\operatorname { R e }}$, with a $\% \Delta H$ of 5.52 . Unlike the 
best AIC from the prior search, this model includes esophageal reflux (Rer) and excludes hypopotassemia (Rhp).

\section{- Comparing Search Results (Hip, Re)}

All IVs and the Comorbidity IVs searches yield 2 sets of results that can be compared to each other and interpreted into a 3-tiered classification of results, as follows.

\section{Table 66. The 3-Tiered Classification of Predicting} Variables for DV: Readmission, Hip Analysis.

\begin{tabular}{|l|l|}
\hline Tier & Variables \\
\hline Tier 1 (Most Important) & *Independence model \\
\hline Tier 2 & None \\
\hline Tier 3 & Rhp, Rdd, Rpa, Rep, Rhe, Rys, Rki \\
\hline
\end{tabular}

Tier 1 contains variables from the best-by-BIC model from the dataset All IVs. This is the most complete search, and the one that provides the most conservative predictors. In the Readmission analysis for Hip, there were no predictors better than the independence model according to BIC.

Tier 2 contains variables not in Tier1, but found in the AIC (less conservative) model of All IVs AND in the BIC model of Comorbidity IVs. For the Hip Analysis of DV Readmission, there were no variables that remained.

Finally, this classification places into Tier 3 any variables present elsewhere in the best model search results, but not included in Tier 1 or Tier 2 ; that is, any variables unique to one of the two searches_-variables in the AIC model of All IVs but not in the BIC model of Comorbidity IVs, and variables in the BIC model of Comorbidity IVs but not in the AIC model from All IVs. Tier 3 is the only classification that shows the predictors for Readmission (Rhp, Rdd, Rpa, Rep, Rhe, Rys, and Rki). 


\section{- Model FIT}

The detailed content of the best fine-grained AIC model from the All IVs search, Rhp $\mathbf{R e}: \operatorname{Rdd} \mathbf{R e}: \operatorname{Rpa} \mathbf{R e}: \operatorname{Rep} \mathbf{R e}:$ Rhe $\mathbf{R e}:$ Rys $\mathbf{R e}:$ Rki $\mathbf{R e}$, is shown as a conditional probability distribution in Table 67.

Table 67. Fit Table (Hip) for All IVs for Best Model Rhp Re : Rdd Re : Rpa Re : Rep Re : Rhe Re : Rys Re : Rki Re.

Blue rows indicate ratio $<0.90$ and orange rows indicate ratio $>1.10$.

(Frequencies $<10$ and ratios between 0.91 and 1.09 are close to the margins and are excluded.)

\begin{tabular}{|c|c|c|c|c|c|c|c|c|c|c|c|c|c|c|}
\hline & \multicolumn{7}{|l|}{ IVs } & \multicolumn{3}{|l|}{ Data } & \multirow{2}{*}{\multicolumn{2}{|c|}{ Model }} & \multirow[b]{3}{*}{ ratio } & \multirow[b]{3}{*}{$\mathrm{p}($ margin $)$} \\
\hline & & & & & & & & & obs. p & DV|IV) & & & & \\
\hline \# & Rhp & Rdd & Rpa & Rep & Rhe & Rys & Rki & freq & $\mathrm{Re}=0$ & $\mathrm{Re}=1$ & $\mathrm{Re}=0$ & $\mathrm{Re}=1$ & & \\
\hline 1 & 0 & 0 & 0 & 0 & 0 & 0 & 0 & 1680 & 98.10 & 1.91 & 98.26 & 1.75 & 0.64 & 0.01 \\
\hline 2 & 0 & 0 & 0 & 1 & 0 & 0 & 0 & 12 & 91.67 & 8.33 & 86.89 & 13.11 & 4.82 & 0.03 \\
\hline 3 & 0 & 1 & 0 & 0 & 1 & 0 & 0 & 27 & 81.48 & 18.52 & 87.04 & 12.96 & 4.77 & 0.00 \\
\hline & & & & & & & & 3200 & 97.28 & 2.72 & 97.28 & 2.72 & 1 & \\
\hline
\end{tabular}

This resulted in a conditional probability distribution where after deleting rows with $\mathrm{p}>0.05$ and freq $<10$, only three rows remained. Table 67 and the following decision tree in Figure 13 show that when none of the comorbidities are present, there is a protective effect relative to the margins with a risk of readmission at $1.75 \%$ and a risk ratio of 0.64 , compared to $2.72 \%$ observed in the data. When epilepsy (Rep) alone is present, or dysthymic disorder (Rdd) along with essential hypertension (Rhe), the risk increases to $13.11 \%$ and $12.96 \%$ (ratio 4.82 and 4.77 ). 


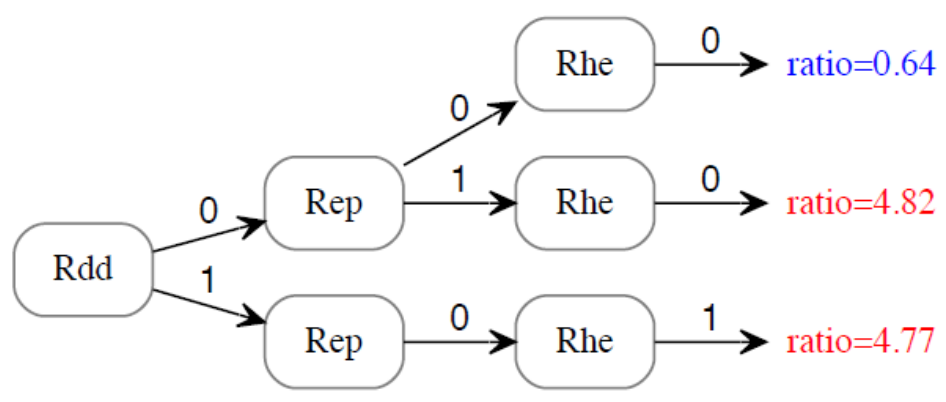

Figure 13. Decision Tree for DV Readmit (Hip) All IVs for Best AIC Model Rhp Re : Rdd Re : Rpa Re : Rep Re : Rhe Re : Rys Re : Rki Re.

The individual components of the model each have a fit table, condensed into a single table below (Table 68).

Table 68. Condensed Fit Table of Components (Hip) All IVs Best Model Rhp Re : Rdd Re : Rpa Re : Rep Re : Rhe Re : Rys Re : Rki Re. Orange rows indicate ratio $>\mathbf{1 . 1 0}$. (Frequencies $<10$ are indicated in gray).

\begin{tabular}{|c|c|c|c|c|c|c|c|}
\hline & & & Data & \multicolumn{2}{|c|}{ obs. p(DV|IV) } & & \\
\hline$\#$ & IV & State & freq & Re=0 & Re=1 & ratio & p(margin) \\
\hline 1 & Rhp & 1 & 7 & 71.43 & 28.57 & 10.51 & 0.00 \\
\hline 2 & Rdd & 1 & 58 & 89.66 & 10.35 & 3.80 & 0.00 \\
\hline 3 & Rpa & 1 & 9 & 77.78 & 22.22 & 8.17 & 0.00 \\
\hline 4 & Rep & 1 & 19 & 84.21 & 15.79 & 5.81 & 0.00 \\
\hline 5 & Rhe & 1 & 1462 & 96.58 & 3.42 & 1.26 & 0.10 \\
\hline 6 & Rys & 1 & 7 & 71.43 & 28.57 & 10.51 & 0.00 \\
\hline 7 & Rki & 1 & 6 & 66.67 & 33.33 & 12.26 & 0.00 \\
\hline & & & 3200 & 97.28 & 2.72 & 1.00 & \\
\hline
\end{tabular}

The Comorbidity IVs model, Rdd $\mathbf{R e}:$ Rpa $\mathbf{R e}: \operatorname{Rep} \mathbf{R e}:$ Rhe $\mathbf{R e}: \operatorname{Rys} \mathbf{R e}:$

Rer Re : Rki Re, resulted in a conditional probability distribution where after deleting rows with $\mathrm{p}>0.05$ and freq $<10$, only two rows remained (Table 69). 
Table 69. Fit Table (Hip) for Comorbidity IVs for Best Model Rdd Re :

Rpa Re : Rep Re : Rhe Re : Rys Re : Rer Re : Rki Re.

Blue rows indicate ratio $<0.90$ and orange rows indicate ratio $>1.10$.

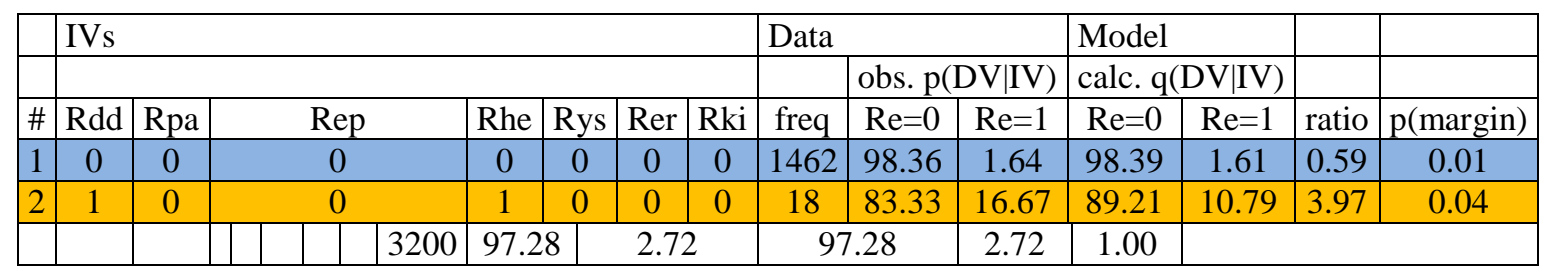

The decision tree providers a very simple explanation where the occurrence of dysthymic disorder (Rdd) along with essential hypertension (Rhe) result in increased risk of readmission with a ratio of 3.97 , and the absence of the comorbidities result in a reduced risk with ratio 0.59 .

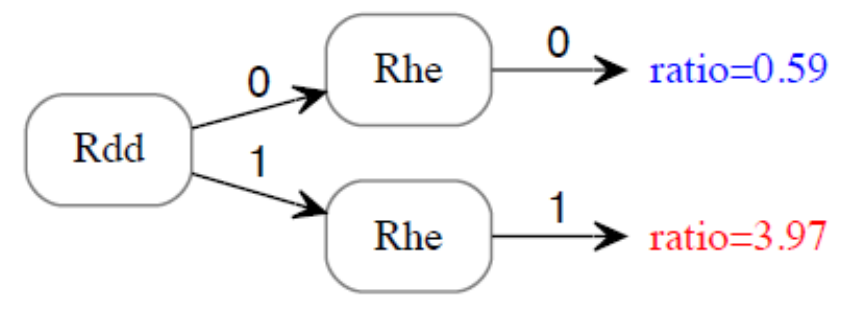

Figure 14. Decision Tree for DV Readmit (Hip) with Comorbidity IVs for Best AIC Model Rdd Re : Rpa Re : Rep Re : Rhe Re : Rys Re : Rer Re : Rki Re Re.

The individual components of the model each have a fit table, condensed into a single table below (Table 70). 
Table 70. Condensed Fit Table of Components (Hip) All IVs

Best AIC Model Rdd Re : Rpa Re : Rep Re : Rhe Re : Rys Re : Rer Re : Rki Re Re. Orange rows indicate ratio $>1.10$. (Frequencies $<10$ are indicated in gray).

\begin{tabular}{|c|c|c|c|c|c|c|c|}
\hline & & & Data & \multicolumn{2}{|c|}{ obs. p(DV|IV) } & & \\
\hline$\#$ & IV & State & freq & Re=0 & $\operatorname{Re}=1$ & ratio & $\mathrm{p}$ (margin) \\
\hline 1 & Rdd & 1 & 58 & 89.66 & 10.35 & 3.80 & 0.00 \\
\hline 2 & Rpa & 1 & 9 & 77.78 & 22.22 & 8.17 & 0.00 \\
\hline 3 & Rep & 1 & 19 & 84.21 & 15.79 & 5.81 & 0.00 \\
\hline 4 & Rhe & 1 & 1462 & 96.58 & 3.42 & 1.26 & 0.10 \\
\hline 5 & Rys & 1 & 7 & 71.43 & 28.57 & 10.51 & 0.00 \\
\hline 6 & Rer & 1 & 554 & 95.49 & 4.51 & 1.66 & 0.01 \\
\hline 7 & Rki & 1 & 6 & 66.67 & 33.33 & 12.26 & 0.00 \\
\hline & & & 3200 & 97.28 & 2.72 & 1.00 & \\
\hline
\end{tabular}

\section{Hip \& Knee Summary for Readmissions}

The most important IVs are summarized in Table 71 . The only IV that shows up in both searches is epilepsy (Rep).

Table 71. Summary of Most Important IVs by Tier Across Hip and Knee for Readmission.

\begin{tabular}{|c|l|l|}
\hline & \multicolumn{2}{|c|}{ Readmission (Re) } \\
\hline Tier & Knee & Hip \\
\hline 1 & Rci, Rco, Rgp, Rcj & *Independence model \\
\hline 2 & None & None \\
\hline 3 & Rep, Rca, Rdf & Rhp, Rdd, Rpa, Rep, Rhe, Rys, Rki \\
\hline
\end{tabular}

\section{Total Cost (Tcb)}

Knee Analysis

- All IVs

- Coarse Searches (Models without Loops)

After examining loopless models for the dependent variable Total Cost Binned (Tcb), single predicting variables with $\mathrm{p} \leq 0.05$ were retained, resulting in 57 IVs to keep for the next round of searches. The reduced data set contained 68 IVs in total, of which 
57 were individually predictive with $\mathrm{p} \leq 0.05$. Of the 23 literature-based IVs, 11 were not found individually predictive but were retained regardless, and 12 literature-based IVs were found to be in common with the individually predictive IVs. The top 10 predicting variables are below in Table 72 .

Table 72. Summary of Search Results (Knee) for All IVs for Total Cost Binned (Tcb). Search covers coarse and fine models.

\begin{tabular}{|c|c|c|c|c|c|}
\hline MODEL & $\Delta \mathrm{df}$ & $\Delta \mathrm{BIC}$ & $\% \Delta \mathrm{H}$ & Alpha & Variable description \\
\hline \multicolumn{6}{|c|}{ COARSE, single predictors (top 10) } \\
\hline S Tcb & 124 & 1220.44 & 23.71 & 0.00 & Surgeon \\
\hline L Tcb & 12 & 408.12 & 5.34 & 0.00 & Location \\
\hline Svb Tcb & 4 & 406.28 & 4.62 & 0.00 & Surgeon volume binned \\
\hline Da Tcb & 8 & 200.53 & 2.81 & 0.00 & Day of admit \\
\hline Ad Tcb & 54 & -190.79 & 2.74 & 0.00 & Admit diagnosis \\
\hline $\mathrm{Nrb}$ Tcb & 4 & 140.10 & 1.82 & 0.00 & Number of risks binned \\
\hline Rmo Tcb & 2 & 31.17 & 0.50 & 0.00 & Morbid obesity (278.01) \\
\hline Ros Tcb & 2 & 19.72 & 0.38 & 0.00 & Obstructive sleep apnea (327.23) \\
\hline Rde Tcb & 2 & 17.37 & 0.36 & 0.00 & Depressive disorder (311) \\
\hline Ruh Tcb & 2 & 12.95 & 0.31 & 0.00 & Hyperlipidemia (272.4) \\
\hline MODEL & $\Delta \mathrm{df}$ & $\triangle \mathrm{BIC}$ & $\% \Delta \mathrm{H}$ & Alpha & Variable description \\
\hline \multicolumn{6}{|c|}{ COARSE, IVs in AIC or BIC models but not in top 10} \\
\hline Rht Tcb (rank 29) & 2 & -6.68 & 0.11 & 0.01 & Heart disease (429.9) \\
\hline Rnn Tcb (rank 30) & 2 & -7.00 & 0.10 & 0.01 & Nephritis and nephropathy (583.81) \\
\hline Rmn Tcb (rank 40) & 2 & -8.16 & 0.09 & 0.01 & Mononeuritis (355.9) \\
\hline Rug Tcb (rank 46) & 2 & -9.01 & 0.08 & 0.02 & Glaucoma (365.9) \\
\hline MODEL & $\Delta \mathrm{df}$ & $\triangle \mathrm{BIC}$ & $\% \Delta \mathrm{H}$ & Alpha & Variable description \\
\hline \multicolumn{6}{|c|}{ COARSE, best model (loopless) } \\
\hline \multicolumn{6}{|c|}{$\Delta \mathrm{BIC}$, Inc.P \& $\triangle \mathrm{AIC} \quad$ (same best model) } \\
\hline S Tcb & 124 & 1220.44 & 23.71 & 0.00 & Surgeon \\
\hline MODEL & $\Delta \mathrm{df}$ & $\Delta \mathrm{BIC}$ & $\% \Delta \mathrm{H}$ & Alpha & Variable description \\
\hline \multicolumn{6}{|c|}{ FINE, best models (with loops) } \\
\hline \multicolumn{6}{|c|}{$\Delta \mathrm{BIC}$ (best model) } \\
\hline S Tcb : Nrb Tcb & 128 & 1322.66 & 25.13 & 0.00 & Surgeon, Number of Risks Binned \\
\hline \multicolumn{6}{|c|}{ Inc.P \& $\triangle \mathrm{AIC}$ (same best model) } \\
\hline $\begin{array}{l}\text { S Tcb : Nrb Tcb : } \\
\text { Rmo Tcb : Rmn Tcb } \\
\text { Rug Tcb : Rht Tcb : } \\
\text { Rnn Tcb }\end{array}$ & 138 & 1296.32 & 25.74 & 0.01 & $\begin{array}{l}\text { Surgeon, Number of Risks Binned, } \\
\text { Morbid obesity (278.01), Mononeuritis } \\
\text { (355.9), Glaucoma (365.9), Heart disease } \\
\text { (429.9), Nephritis and nephropathy } \\
\text { (583.81) }\end{array}$ \\
\hline
\end{tabular}

Simply knowing the surgeon $(\mathrm{S})$ reduces the uncertainty in predicting Total Cost

Binned (Tcb) by $23.71 \%$ (Table 72 ). This is a very substantial $\% \Delta \mathrm{H}$, the relevance of which will be discussed further in the discussion chapter. Because surgeon (S) has 64 
possible states, the $\Delta \mathrm{df}$ is quite large. Location (L) is the next most individually predictive, with a $\% \Delta \mathrm{H}$ of 5.34 , followed by surgeon volume binned $(\mathrm{Svb})$ with $\% \Delta \mathrm{H}$ at 4.62. In fact, the most predictive single variables with a $\% \Delta \mathrm{H}$ greater than $1 \%$ are in All IVs. Day of admit (Da), admit diagnosis (Ad), and number of risks binned (Nrb) all have $\% \Delta \mathrm{H}>1$ at $2.81,2.74$, and 1.82 respectively (Table 72 ). In coarse searches that allow for more than one predicting IV, the best model, S Tcb, still has only one predictor, namely the top single predicting variable S.

\section{- Fine Searches (Models with Loops)}

The fine-grained search allows for multiple components as well as interaction effects, but none were found in any of the fine-grained models, regardless of selection criteria used for picking the best model. There is an improvement in $\% \Delta \mathrm{H}$ with the addition of number of risks binned ( $\mathrm{Nrb}$ ), resulting in the best fine-grained model by BIC of S Tcb : Nrb Tcb with a $\% \Delta \mathrm{H}$ of 25.13 . In the single predicting variable search, $\mathrm{Nrb}$ was the sixth most individually predictive, but in the fine-grained model Nrb added the most information relative to its added complexity than the four All IVs that were individually more predictive of Tcb (L, Svb, Da, and Ad).

- Comorbidity IVs

- Coarse Searches (Models without Loops)

The most predictive comorbidity, in the Comorbidity IVs search is morbid obesity (Rmo), which was the seventh most predictive individual variable with a $\% \Delta \mathrm{H}$ of 0.50 . Obstructive sleep apnea (Ros), depressive disorder (Rde), and hyperlipidemia (Ruh) are then the next most predictive Comorbidity IVs, but with relatively small predictive value 
on Tcb compared to the All IVs. Additional predictive single Comorbidity IVs are listed in Table 73.

Table 73. Summary of Search Results (Knee) for Comorbidity IVs for DV Total Cost Binned (Tcb). Search covers coarse and fine models.

All $\mathbf{p}$ values $=\mathbf{0}$.

\begin{tabular}{|c|c|c|c|c|}
\hline MODEL & $\Delta \mathrm{df}$ & $\Delta \mathrm{BIC}$ & $\% \Delta \mathrm{H}$ & Variable description \\
\hline \multicolumn{5}{|c|}{ COARSE, single predictors } \\
\hline Rmo Tcb & 2 & 31.17 & 0.50 & Morbid obesity (278.01) \\
\hline Ros Tcb & 2 & 19.72 & 0.38 & Obstructive sleep apnea (327.23) \\
\hline Rde Tcb & 2 & 17.37 & 0.36 & Depressive disorder (311) \\
\hline Ruh Tcb & 2 & 12.95 & 0.31 & Hyperlipidemia (272.4) \\
\hline Rrd Tcb & 2 & 7.54 & 0.25 & Hypertensive renal disease (403.9) \\
\hline Rcp Tcb & 2 & 5.55 & 0.23 & Chronic pain (338.29) \\
\hline Rad Tcb & 2 & 5.11 & 0.23 & Anxiety, dissociative and somatoform disorders (300) \\
\hline Rer Tcb & 2 & 4.44 & 0.22 & Esophageal reflux (530.81) \\
\hline Rhe Tcb & 2 & 0.29 & 0.18 & Essential hypertension (401.9) \\
\hline Rdi Tcb & 2 & -0.01 & 0.18 & Diabetes mellitus (250) \\
\hline MODEL & $\Delta \mathrm{df}$ & $\Delta \mathrm{BIC}$ & $\% \Delta \mathrm{H}$ & Variable description \\
\hline \multicolumn{5}{|c|}{ COARSE, best model (loopless) } \\
\hline \multicolumn{5}{|c|}{$\Delta \mathrm{BIC}$ (best model) } \\
\hline Rmo Tcb & 2 & 31.17 & 0.50 & Morbid obesity (278.01) \\
\hline \multicolumn{5}{|l|}{$\Delta$ AIC (best model) } \\
\hline Ruh Rmo Rde Ros Tcb & 30 & -104.39 & 1.54 & $\begin{array}{l}\text { Hyperlipidemia }(272.4)+\text { Morbid obesity }(278.01)+ \\
\text { Depressive disorder }(311)+\text { Obstructive sleep apnea } \\
(327.23)\end{array}$ \\
\hline \multicolumn{5}{|l|}{ Inc.P (best model) } \\
\hline $\begin{array}{l}\text { Ruh Rmo Rde Ros Rhe } \\
\text { Tcb }\end{array}$ & 62 & -319.60 & 2.10 & $\begin{array}{l}\text { Hyperlipidemia }(272.4)+\text { Morbid obesity }(278.01)+ \\
\text { Depressive disorder }(311)+\text { Obstructive sleep apnea } \\
(327.23)+\text { Essential hypertension }(401.9)\end{array}$ \\
\hline MODEL & $\Delta \mathrm{df}$ & $\Delta \mathrm{BIC}$ & $\% \Delta \mathrm{H}$ & Variable description \\
\hline \multicolumn{5}{|c|}{ FINE, best models (with loops) } \\
\hline \multicolumn{5}{|c|}{$\Delta \mathrm{BIC}$ (best model) } \\
\hline $\begin{array}{l}\text { Ruh Tcb : Rmo Tcb : } \\
\text { Rde Tcb : Ros Tcb : } \\
\text { Rhe Tcb }\end{array}$ & 10 & 60.11 & 1.51 & $\begin{array}{l}\text { Hyperlipidemia }(272.4)+\text { Morbid obesity }(278.01)+ \\
\text { Depressive disorder }(311)+\text { Obstructive sleep apnea } \\
(327.23)+\text { Essential hypertension }(401.9)\end{array}$ \\
\hline \multicolumn{5}{|c|}{ Inc.P \& $\triangle \mathrm{AIC}$ (same best model) } \\
\hline $\begin{array}{l}\text { Ruh Tcb : Rmo Tcb : } \\
\text { Rad Tcb : Rde Tcb : } \\
\text { Ros Tcb : Rcp Tcb : } \\
\text { Rhe Tcb }\end{array}$ & 14 & 56.49 & 1.82 & $\begin{array}{l}\text { Hyperlipidemia (272.4) + Morbid obesity (278.01)+ } \\
\text { Anxiety, dissociative and somatoform disorders (300) + } \\
\text { Depressive disorder (311) + Obstructive sleep apnea } \\
\text { (327.23) + Chronic pain (338.29) + Essential } \\
\text { hypertension (401.9) }\end{array}$ \\
\hline
\end{tabular}

- Fine Searches (Models with Loops)

The best model by BIC for Comorbidity IVs is Ruh Tcb : Rmo Tcb : Rde Tcb :

Ros Tcb : Rhe Tcb, with a $\% \Delta H$ of 1.51 (Table 73) and a $\Delta$ df of 10. 


\section{- Comparing Search Results (Knee, Tcb)}

The All IVs and the Comorbidity IVs searches yield two sets of results that can be compared to each other and interpreted with a three-tiered classification of results, as described below and summarized in Table 74.

Table 74. The 3-Tiered Classification of Predicting Variables for DV: Total Cost, Knee Analysis

\begin{tabular}{|l|l|}
\hline Tier & Variables \\
\hline Tier 1-Most Important & S, Nrb \\
\hline Tier 2 & Rmo \\
\hline Tier 3 & Rmn, Rug, Rht, Rnn, Ruh, Rde, Ros, Rhe \\
\hline
\end{tabular}

Tier 1 contains variables from the best-by-BIC model from the dataset All IVs. This is the most complete search, and the one that provides the most conservative predictors. The selected variables $(\mathrm{S}, \mathrm{Nrb})$ are shown in the first row of Table 74, and are considered the most important predicting variables.

Tier 2 contains variables not in Tier1, but found in the AIC (less conservative) model of All IVs AND in the BIC model of Comorbidity IVs. For the Knee Analysis of DV Total Cost Binned (Tcb), this selects the variable Rmo as the next-most important predicting variable. These are shown in the second row of Table 74.

Finally, this classification places into Tier 3 any variables present elsewhere in the best model search results, but not included in Tier 1 or Tier 2 ; that is, any variables unique to one of the two searches_-variables in the AIC model of All IVs but not in the BIC model of Comorbidity IVs, and variables in the BIC model of Comorbidity IVs but not in the AIC model from All IVs. This criterion shows the next most important predicting variables (Rmn, Rug, Rht, Rnn, Ruh, Rde, Ros, and Rhe) as seen in row 3 of Table 74. 


\section{- Model FIT}

The detailed content of the best BIC model from the All IVs search, S Tcb : Nrb

Tcb, is shown as a conditional probability distribution in Table 76 below. The values for

Total Cost were discretized into 3 bins. The bin ranges, averages, and frequencies are

below in Table 75 .

Table 75. Bin values for DV Total Cost.

\begin{tabular}{|c|c|c|c|c|}
\hline Bin & Min Cost & Max Cost & Average Cost & Frequency \\
\hline 1 & $\$ 8,553$ & $\$ 16,780$ & $\$ 15,269$ & 1446 \\
\hline 2 & $\$ 16,781$ & $\$ 19,139$ & $\$ 17,922$ & 1445 \\
\hline 3 & $\$ 19,140$ & $\$ 96,880$ & $\$ 22,318$ & 1445 \\
\hline
\end{tabular}

In the conditional probability distribution below, the ratio is based on the

Expected Value column. Here, the ratio is calculated by taking the expected cost for a

row and dividing it by the expected cost for the marginal distribution.

Table 76. Full Fit Table (Knee) All IVs for Best Model: S Tcb : Nrb Tcb.

Blue rows indicate ratio $<0.90$ and orange rows indicate ratio $>1.10$

(ratios between 0.91 and 1.09 are close to the margins and are indicated in gray).

\begin{tabular}{|c|c|c|c|c|c|c|c|c|c|c|c|c|c|c|}
\hline & \multicolumn{1}{|l}{ IVs } & \multicolumn{1}{|c|}{ Data } & \multicolumn{1}{|l|}{ Model } & & & & \\
\hline & & & & \multicolumn{3}{|c|}{ obs. $\mathrm{pV} \mid \mathrm{IV})$} & \multicolumn{2}{c|}{ calc. q(DV|IV) } & & & \\
\hline$\#$ & $\mathrm{~S}$ & $\mathrm{Nrb}$ & freq & $\mathrm{Tcb}=1$ & $\mathrm{Tcb}=2$ & $\mathrm{Tcb}=3$ & $\mathrm{Tcb}=1$ & $\mathrm{Tcb}=2$ & $\mathrm{Tcb}=3$ & Exp. Value & Ratio & rule & p(margin) \\
\hline 1 & 1 & 1 & 32 & 96.88 & 3.13 & 0.00 & 96.84 & 3.16 & 0.00 & $\$ 15,353.04$ & 0.83 & 1 & 0.00 \\
\hline 2 & 1 & 2 & 21 & 100.00 & 0.00 & 0.00 & 95.66 & 4.34 & 0.00 & $\$ 15,384.34$ & 0.83 & 1 & 0.00 \\
\hline 3 & 1 & 3 & 16 & 87.50 & 12.50 & 0.00 & 93.26 & 6.74 & 0.00 & $\$ 15,448.07$ & 0.83 & 1 & 0.00 \\
\hline 5 & 2 & 2 & 21 & 0.00 & 23.81 & 76.19 & 2.41 & 22.46 & 75.13 & $\$ 21,160.63$ & 1.14 & 3 & 0.00 \\
\hline 6 & 2 & 3 & 24 & 4.17 & 12.50 & 83.33 & 1.01 & 14.98 & 84.01 & $\$ 21,588.06$ & 1.17 & 3 & 0.00 \\
\hline 8 & 4 & 1 & 10 & 0.00 & 40.00 & 60.00 & 0.00 & 36.03 & 63.97 & $\$ 20,733.93$ & 1.12 & 3 & 0.05 \\
\hline 9 & 4 & 2 & 11 & 0.00 & 27.27 & 72.73 & 0.00 & 33.56 & 66.44 & $\$ 20,842.54$ & 1.13 & 3 & 0.04 \\
\hline 10 & 4 & 3 & 16 & 0.00 & 25.00 & 75.00 & 0.00 & 23.16 & 76.84 & $\$ 21,299.87$ & 1.15 & 3 & 0.00 \\
\hline 14 & 8 & 1 & 28 & 67.86 & 25.00 & 7.14 & 71.40 & 22.16 & 6.44 & $\$ 16,311.11$ & 0.88 & 1 & 0.00 \\
\hline 15 & 8 & 2 & 32 & 65.63 & 31.25 & 3.13 & 63.64 & 27.46 & 8.90 & $\$ 16,624.95$ & 0.90 & 1 & 0.00 \\
\hline 17 & 9 & 1 & 32 & 12.50 & 0.00 & 87.50 & 26.00 & 12.45 & 61.55 & $\$ 19,937.71$ & 1.08 & 3 & 0.00 \\
\hline 18 & 9 & 2 & 12 & 33.33 & 25.00 & 41.67 & 18.74 & 12.48 & 68.78 & $\$ 20,448.31$ & 1.11 & 3 & 0.03 \\
\hline 19 & 9 & 3 & 17 & 23.53 & 23.53 & 52.94 & 8.43 & 8.94 & 82.63 & $\$ 21,330.66$ & 1.15 & 3 & 0.00 \\
\hline 24 & 12 & 3 & 21 & 0.00 & 14.29 & 85.71 & 1.57 & 22.62 & 75.81 & $\$ 21,212.89$ & 1.15 & 3 & 0.00 \\
\hline 28 & 14 & 1 & 35 & 51.43 & 37.14 & 11.43 & 56.22 & 34.58 & 9.21 & $\$ 16,835.67$ & 0.91 & 1 & 0.00 \\
\hline 29 & 14 & 2 & 32 & 53.13 & 37.50 & 9.38 & 47.41 & 40.55 & 12.04 & $\$ 17,193.74$ & 0.93 & 1 & 0.04 \\
\hline 32 & 15 & 2 & 37 & 13.51 & 43.24 & 43.24 & 12.82 & 49.56 & 37.62 & $\$ 19,235.69$ & 1.04 & 2 & 0.02 \\
\hline 35 & 16 & 2 & 37 & 10.81 & 35.14 & 54.05 & 14.37 & 34.70 & 50.93 & $\$ 19,780.04$ & 1.07 & 3 & 0.03 \\
\hline 36 & 16 & 3 & 53 & 9.43 & 26.42 & 64.15 & 6.98 & 26.88 & 66.14 & $\$ 20,644.05$ & 1.12 & 3 & 0.00 \\
\hline
\end{tabular}




\begin{tabular}{|c|c|c|c|c|c|c|c|c|c|c|c|c|c|}
\hline 37 & 17 & 1 & 203 & 20.20 & 11.33 & 68.47 & 20.04 & 11.19 & 68.78 & $\$ 20,413.64$ & 1.10 & 3 & 0.00 \\
\hline 41 & 18 & 2 & 95 & 24.21 & 36.84 & 38.95 & 21.23 & 46.99 & 31.78 & $\$ 18,756.09$ & 1.01 & 2 & 0.01 \\
\hline 42 & 18 & 3 & 98 & 13.27 & 47.96 & 38.78 & 11.73 & 41.37 & 46.91 & $\$ 19,672.84$ & 1.06 & 3 & 0.00 \\
\hline 43 & 19 & 1 & 23 & 65.22 & 30.44 & 4.35 & 73.04 & 21.17 & 5.79 & $\$ 16,239.04$ & 0.88 & 1 & 0.00 \\
\hline 44 & 19 & 2 & 29 & 72.41 & 20.69 & 6.90 & 65.54 & 26.41 & 8.06 & $\$ 16,537.72$ & 0.89 & 1 & 0.00 \\
\hline 47 & 21 & 1 & 41 & 2.44 & 51.22 & 46.34 & 6.82 & 59.03 & 34.15 & $\$ 19,242.29$ & 1.04 & 2 & 0.00 \\
\hline 48 & 21 & 2 & 73 & 6.85 & 56.16 & 36.99 & 4.81 & 57.86 & 37.33 & $\$ 19,435.38$ & 1.05 & 2 & 0.00 \\
\hline 49 & 21 & 3 & 110 & 2.73 & 50.91 & 46.36 & 2.45 & 46.87 & 50.69 & $\$ 20,085.28$ & 1.09 & 3 & 0.00 \\
\hline 53 & 23 & 1 & 19 & 0.00 & 42.11 & 57.90 & 0.00 & 42.12 & 57.89 & $\$ 20,466.61$ & 1.11 & 3 & 0.01 \\
\hline 54 & 24 & 1 & 24 & 12.50 & 62.50 & 25.00 & 8.85 & 45.80 & 45.35 & $\$ 19,680.98$ & 1.06 & 2 & 0.05 \\
\hline 55 & 24 & 2 & 34 & 5.88 & 41.18 & 52.94 & 6.19 & 44.58 & 49.22 & $\$ 19,921.44$ & 1.08 & 3 & 0.00 \\
\hline 56 & 24 & 3 & 26 & 0.00 & 23.08 & 76.92 & 2.97 & 34.04 & 63.00 & $\$ 20,612.53$ & 1.11 & 3 & 0.00 \\
\hline 58 & 26 & 1 & 30 & 6.67 & 56.67 & 36.67 & 1.91 & 59.77 & 38.32 & $\$ 19,555.86$ & 1.06 & 2 & 0.00 \\
\hline 59 & 26 & 2 & 67 & 0.00 & 68.66 & 31.34 & 1.32 & 57.54 & 41.13 & $\$ 19,695.28$ & 1.06 & 2 & 0.00 \\
\hline 60 & 26 & 3 & 83 & 0.00 & 37.35 & 62.65 & 0.65 & 45.19 & 54.15 & $\$ 20,285.32$ & 1.10 & 3 & 0.00 \\
\hline 61 & 27 & 1 & 44 & 18.18 & 72.73 & 9.09 & 31.31 & 60.27 & 8.42 & $\$ 17,461.73$ & 0.94 & 2 & 0.00 \\
\hline 62 & 27 & 2 & 47 & 27.66 & 57.45 & 14.89 & 24.43 & 65.38 & 10.19 & $\$ 17,722.09$ & 0.96 & 2 & 0.00 \\
\hline 63 & 27 & 3 & 43 & 25.58 & 62.79 & 11.63 & 15.68 & 66.86 & 17.46 & $\$ 18,273.95$ & 0.99 & 2 & 0.00 \\
\hline 73 & 32 & 1 & 50 & 96.00 & 4.00 & 0.00 & 90.10 & 7.13 & 2.77 & $\$ 15,653.51$ & 0.85 & 1 & 0.00 \\
\hline 74 & 32 & 2 & 38 & 89.47 & 5.26 & 5.26 & 86.38 & 9.50 & 4.12 & $\$ 15,811.37$ & 0.85 & 1 & 0.00 \\
\hline 75 & 32 & 3 & 21 & 57.14 & 28.57 & 14.29 & 76.77 & 13.46 & 9.77 & $\$ 16,314.99$ & 0.88 & 1 & 0.00 \\
\hline 79 & 35 & 1 & 48 & 85.42 & 12.50 & 2.08 & 74.95 & 20.74 & 4.31 & $\$ 16,123.06$ & 0.87 & 1 & 0.00 \\
\hline 80 & 35 & 2 & 84 & 66.67 & 27.38 & 5.95 & 67.85 & 26.10 & 6.05 & $\$ 16,387.88$ & 0.89 & 1 & 0.00 \\
\hline 81 & 35 & 3 & 100 & 50.00 & 36.00 & 14.00 & 54.02 & 33.12 & 12.86 & $\$ 17,054.47$ & 0.92 & 1 & 0.00 \\
\hline 87 & 37 & 3 & 34 & 5.88 & 41.18 & 52.94 & 5.51 & 36.62 & 57.88 & $\$ 20,320.42$ & 1.10 & 3 & 0.00 \\
\hline 90 & 38 & 3 & 18 & 5.56 & 5.56 & 88.89 & 4.48 & 2.99 & 92.53 & $\$ 21,870.39$ & 1.18 & 3 & 0.00 \\
\hline 96 & 41 & 1 & 163 & 73.01 & 22.09 & 4.91 & 69.06 & 23.09 & 7.84 & $\$ 16,434.82$ & 0.89 & 1 & 0.00 \\
\hline 97 & 41 & 2 & 190 & 60.00 & 29.47 & 10.53 & 60.94 & 28.33 & 10.73 & $\$ 16,777.31$ & 0.91 & 1 & 0.00 \\
\hline 98 & 41 & 3 & 112 & 41.07 & 33.04 & 25.89 & 45.22 & 33.51 & 21.27 & $\$ 17,657.72$ & 0.95 & 1 & 0.01 \\
\hline 101 & 44 & 1 & 146 & 70.55 & 27.40 & 2.06 & 72.01 & 23.99 & 4.01 & $\$ 16,187.92$ & 0.87 & 1 & 0.00 \\
\hline 102 & 44 & 2 & 217 & 62.21 & 32.26 & 5.53 & 64.54 & 29.89 & 5.57 & $\$ 16,454.65$ & 0.89 & 1 & 0.00 \\
\hline 103 & 44 & 3 & 163 & 55.22 & 31.29 & 13.50 & 50.80 & 37.50 & 11.70 & $\$ 17,089.05$ & 0.92 & 1 & 0.00 \\
\hline 105 & 45 & 2 & 68 & 29.41 & 50.00 & 20.59 & 27.15 & 52.44 & 20.40 & $\$ 18,098.80$ & 0.98 & 2 & 0.00 \\
\hline 106 & 45 & 3 & 81 & 14.82 & 55.56 & 29.63 & 16.43 & 50.58 & 32.99 & $\$ 18,936.35$ & 1.02 & 2 & 0.00 \\
\hline 115 & 49 & 3 & 36 & 11.11 & 38.89 & 50.00 & 8.92 & 29.92 & 61.17 & $\$ 20,374.21$ & 1.10 & 3 & 0.00 \\
\hline 118 & 51 & 2 & 12 & 0.00 & 0.00 & 100.00 & 0.00 & 6.58 & 93.42 & $\$ 22,028.56$ & 1.19 & 3 & 0.00 \\
\hline 119 & 51 & 3 & 44 & 0.00 & 6.82 & 93.18 & 0.00 & 4.03 & 95.97 & $\$ 22,140.51$ & 1.20 & 3 & 0.00 \\
\hline 120 & 52 & 1 & 15 & 0.00 & 20.00 & 80.00 & 4.92 & 31.97 & 63.11 & $\$ 20,565.84$ & 1.11 & 3 & 0.03 \\
\hline 121 & 52 & 2 & 22 & 0.00 & 27.27 & 72.73 & 3.34 & 30.19 & 66.47 & $\$ 20,755.05$ & 1.12 & 3 & 0.00 \\
\hline 122 & 52 & 3 & 36 & 5.56 & 27.78 & 66.67 & 1.46 & 21.01 & 77.53 & $\$ 21,291.19$ & 1.15 & 3 & 0.00 \\
\hline 127 & 54 & 3 & 72 & 19.44 & 27.78 & 52.78 & 21.13 & 31.01 & 47.86 & $\$ 19,465.04$ & 1.05 & 3 & 0.02 \\
\hline 142 & 60 & 2 & 17 & 0.00 & 11.77 & 88.24 & 2.76 & 16.75 & 80.49 & $\$ 21,387.00$ & 1.16 & 3 & 0.00 \\
\hline 143 & 60 & 3 & 14 & 0.00 & 14.29 & 85.71 & 1.13 & 10.92 & 87.95 & $\$ 21,758.27$ & 1.18 & 3 & 0.00 \\
\hline 149 & 63 & 1 & 33 & 0.00 & 27.27 & 72.73 & 2.09 & 32.63 & 65.28 & $\$ 20,736.38$ & 1.12 & 3 & 0.00 \\
\hline 150 & 63 & 2 & 62 & 3.23 & 33.87 & 62.90 & 1.40 & 30.52 & 68.08 & $\$ 20,877.44$ & 1.13 & 3 & 0.00 \\
\hline \multirow[t]{2}{*}{151} & 63 & 3 & 73 & 0.00 & 20.55 & 79.45 & 0.61 & 20.97 & 78.42 & $\$ 21,353.26$ & 1.15 & 3 & 0.00 \\
\hline & & & 4336 & 33.35 & 33.33 & 33.33 & 33.35 & 33.33 & 33.33 & $\$ 18,502.55$ & 1.00 & 1 & \\
\hline \# & $\mathrm{S}$ & $\mathrm{Nrb}$ & freq & $\mathrm{Tcb}=1$ & $\mathrm{Tcb}=2$ & $\mathrm{Tcb}=3$ & $\mathrm{Tcb}=1$ & $\mathrm{Tcb}=2$ & $\mathrm{Tcb}=3$ & Exp. Value & Ratio & rule & $p($ margin $)$ \\
\hline
\end{tabular}

- 156 - 
Surgeon 1, regardless of the number of risks ( $\mathrm{Nrb}$ ) of the patient, consistently is expected to have low cost. This is shown in rows 1,2, and 3 of Table 76. Surgeons 8 and 9 also have low expected total cost, although their cases both had Nrb in states 1 or 2 (rows 14-18). Surgeons 32 and 35, regardless of Nrb state also are predicted to have low expected cost (rows 73-81), although there is an increase in expected cost as the number of risks increase. Surgeons 41 and 44 also have consistently low expected costs regardless of Nrb (rows 96-103), although a couple of these rows have risk ratio under, but close to 1 . Surgeons 52 and 63 (rows 120-122 and 149-151, respectively) have high expected total cost regardless of $\mathrm{Nrb}$ state (Table 76).

Each component of the model is interesting, particularly in the analysis of Tcb. In this project, the names of the individual surgeons are not included. However, each S state correlates to an individual surgeon. The surgeon component table shows the observed $\mathrm{p}(\mathrm{DV} \mid \mathrm{IV})$. In Table 77, the probability of $\mathrm{Tcb}=1$ given surgeon $(\mathrm{S})=1$ is $95.65 \%$ compared to the margin projections of 33.4\%. Given this surgeon, with 69 knee surgeries in this data set, the Expected Value (the expected total cost) per case is $\$ 15,385$ compared to the average overall for all knee patients at $\$ 18,503$. Surgeon $(S)=2$ has comparable volume (freq $=52$ ) but only $1.92 \%$ of observed cases in the low total cost bin, and just over $19 \%$ in bin 2 . This surgeon has $78.6 \%$ of cases in the high-cost bin, with an expected total cost of $\$ 21,337$. The surgeon (S) with the highest Expected Value is surgeon 51, with 62 surgeries in the data set and an expected total cost of $\$ 22,105$. 
Table 77. Component Fit Table for IV S (Knee) All IVs

Best Model: L Tcb : Svb Tcb : Nrb Tcb.

Blue rows indicate ratio $<0.90$ and orange rows indicate ratio $>1.10$.

(Ratios between 0.91 and 1.09 are close to the margins and are indicated in gray.)

\begin{tabular}{|c|c|c|c|c|c|c|c|c|c|}
\hline \multirow[b]{3}{*}{ \# } & \multirow{3}{*}{$\begin{array}{l}\text { IV } \\
\text { S }\end{array}$} & \multicolumn{4}{|l|}{ Data } & \multirow[b]{3}{*}{ Exp. Value } & \multirow[b]{3}{*}{ Ratio } & \multirow[b]{3}{*}{ rule } & \multirow[b]{3}{*}{$\mathrm{p}$ (margin) } \\
\hline & & & \multicolumn{3}{|c|}{ obs. p(DV|IV) } & & & & \\
\hline & & freq & $\mathrm{Tcb}=1$ & $\mathrm{Tcb}=2$ & $\mathrm{Tcb}=3$ & & & & \\
\hline 1 & 1 & 69 & 95.65 & 4.35 & 0.00 & $\$ 15,384.61$ & 0.83 & 1 & 0.00 \\
\hline 2 & 2 & 52 & 1.92 & 19.23 & 78.85 & $\$ 21,336.88$ & 1.15 & 3 & 0.00 \\
\hline 3 & 3 & 2 & 0.00 & 0.00 & 100.00 & $\$ 22,317.68$ & 1.21 & 3 & 0.14 \\
\hline 4 & 4 & 37 & 0.00 & 29.73 & 70.27 & $\$ 21,010.96$ & 1.14 & 3 & 0.00 \\
\hline 5 & 5 & 3 & 0.00 & 33.33 & 66.67 & $\$ 20,852.60$ & 1.13 & 3 & 0.37 \\
\hline 6 & 7 & 2 & 50.00 & 0.00 & 50.00 & $\$ 18,793.47$ & 1.02 & $* 1$ & 0.61 \\
\hline 7 & 8 & 84 & 61.91 & 27.38 & 10.71 & $\$ 16,750.88$ & 0.91 & 1 & 0.00 \\
\hline 8 & 9 & 61 & 19.67 & 11.48 & 68.85 & $\$ 20,426.53$ & 1.10 & 3 & 0.00 \\
\hline 9 & 10 & 8 & 0.00 & 25.00 & 75.00 & $\$ 21,218.86$ & 1.15 & 3 & 0.03 \\
\hline 10 & 11 & 1 & 100.00 & 0.00 & 0.00 & $\$ 15,269.25$ & 0.83 & 1 & 0.37 \\
\hline 11 & 12 & 37 & 2.70 & 27.03 & 70.27 & $\$ 20,939.25$ & 1.13 & 3 & 0.00 \\
\hline 12 & 13 & 17 & 0.00 & 35.29 & 64.71 & $\$ 20,766.41$ & 1.12 & 3 & 0.01 \\
\hline 13 & 14 & 107 & 44.86 & 40.19 & 14.95 & $\$ 17,389.42$ & 0.94 & 1 & 0.00 \\
\hline 14 & 15 & 78 & 12.82 & 47.44 & 39.74 & $\$ 19,329.28$ & 1.04 & 2 & 0.00 \\
\hline 15 & 16 & 110 & 11.82 & 30.91 & 57.27 & $\$ 20,126.16$ & 1.09 & 3 & 0.00 \\
\hline 16 & 17 & 206 & 19.90 & 11.17 & 68.93 & $\$ 20,424.10$ & 1.10 & 3 & 0.00 \\
\hline 17 & 18 & 244 & 18.85 & 44.26 & 36.89 & $\$ 19,043.25$ & 1.03 & 2 & 0.00 \\
\hline 18 & 19 & 78 & 62.82 & 26.92 & 10.26 & $\$ 16,706.44$ & 0.90 & 1 & 0.00 \\
\hline 19 & 20 & 1 & 0.00 & 100.00 & 0.00 & $\$ 17,922.40$ & 0.97 & 2 & 0.37 \\
\hline 20 & 21 & 224 & 4.02 & 52.68 & 43.30 & $\$ 19,719.31$ & 1.07 & 2 & 0.00 \\
\hline 21 & 22 & 7 & 0.00 & 57.14 & 42.86 & $\$ 19,806.09$ & 1.07 & 2 & 0.20 \\
\hline 22 & 23 & 19 & 0.00 & 42.11 & 57.90 & $\$ 20,467.05$ & 1.11 & 3 & 0.01 \\
\hline 23 & 24 & 84 & 5.95 & 41.67 & 52.38 & $\$ 20,066.78$ & 1.08 & 3 & 0.00 \\
\hline 24 & 25 & 2 & 50.00 & 50.00 & 0.00 & $\$ 16,595.83$ & 0.90 & $* 1$ & 0.61 \\
\hline 25 & 26 & 180 & 1.11 & 52.22 & 46.67 & $\$ 19,944.07$ & 1.08 & 2 & 0.00 \\
\hline 26 & 27 & 134 & 23.88 & 64.18 & 11.94 & $\$ 17,813.60$ & 0.96 & 2 & 0.00 \\
\hline 27 & 28 & 40 & 42.50 & 42.50 & 15.00 & $\$ 17,454.10$ & 0.94 & $* 1$ & 0.05 \\
\hline 28 & 29 & 20 & 0.00 & 50.00 & 50.00 & $\$ 20,120.04$ & 1.09 & $* 2$ & 0.01 \\
\hline 29 & 30 & 3 & 0.00 & 66.67 & 33.33 & $\$ 19,387.48$ & 1.05 & 2 & 0.37 \\
\hline 30 & 31 & 1 & 0.00 & 100.00 & 0.00 & $\$ 17,922.40$ & 0.97 & 2 & 0.37 \\
\hline 31 & 32 & 109 & 86.24 & 9.17 & 4.59 & $\$ 15,835.96$ & 0.86 & 1 & 0.00 \\
\hline 32 & 33 & 5 & 0.00 & 60.00 & 40.00 & $\$ 19,680.51$ & 1.06 & 2 & 0.25 \\
\hline 33 & 34 & 1 & 0.00 & 100.00 & 0.00 & $\$ 17,922.40$ & 0.97 & 2 & 0.37 \\
\hline 34 & 35 & 232 & 63.36 & 28.02 & 8.62 & $\$ 16,620.23$ & 0.90 & 1 & 0.00 \\
\hline 35 & 36 & 35 & 5.71 & 48.57 & 45.71 & $\$ 19,779.88$ & 1.07 & 2 & 0.00 \\
\hline 36 & 37 & 57 & 8.77 & 40.35 & 50.88 & $\$ 19,925.85$ & 1.08 & 3 & 0.00 \\
\hline 37 & 38 & 28 & 7.14 & 3.57 & 89.29 & $\$ 21,657.26$ & 1.17 & 3 & 0.00 \\
\hline 38 & 39 & 40 & 32.50 & 50.00 & 17.50 & $\$ 17,829.30$ & 0.96 & 2 & 0.04 \\
\hline 39 & 40 & 2 & 0.00 & 50.00 & 50.00 & $\$ 20,120.04$ & 1.09 & $* 2$ & 0.61 \\
\hline 40 & 41 & 465 & 60.00 & 27.74 & 12.26 & $\$ 16,869.28$ & 0.91 & 1 & 0.00 \\
\hline 41 & 42 & 1 & 0.00 & 0.00 & 100.00 & $\$ 22,317.68$ & 1.21 & 3 & 0.37 \\
\hline 42 & 43 & 2 & 0.00 & 50.00 & 50.00 & $\$ 20,120.04$ & 1.09 & $* 2$ & 0.61 \\
\hline 43 & 44 & 526 & 62.36 & 30.61 & 7.03 & $\$ 16,576.96$ & 0.90 & 1 & 0.00 \\
\hline 44 & 45 & 187 & 24.06 & 50.80 & 25.13 & $\$ 18,388.66$ & 0.99 & 2 & 0.00 \\
\hline
\end{tabular}




\begin{tabular}{|c|c|c|c|c|c|c|c|c|c|}
\hline 45 & 46 & 4 & 0.00 & 0.00 & 100.00 & $\$ 22,317.68$ & 1.21 & 3 & 0.02 \\
\hline 46 & 47 & 2 & 0.00 & 0.00 & 100.00 & $\$ 22,317.68$ & 1.21 & 3 & 0.14 \\
\hline 47 & 48 & 14 & 28.57 & 50.00 & 21.43 & $\$ 18,106.23$ & 0.98 & 2 & 0.42 \\
\hline 48 & 49 & 61 & 13.12 & 32.79 & 54.10 & $\$ 19,952.20$ & 1.08 & 3 & 0.00 \\
\hline 49 & 50 & 10 & 20.00 & 50.00 & 30.00 & $\$ 18,710.35$ & 1.01 & 2 & 0.50 \\
\hline 50 & 51 & 62 & 0.00 & 4.84 & 95.16 & $\$ 22,104.99$ & 1.19 & 3 & 0.00 \\
\hline 51 & 52 & 73 & 2.74 & 26.03 & 71.23 & $\$ 20,980.59$ & 1.13 & 3 & 0.00 \\
\hline 52 & 53 & 3 & 33.33 & 66.67 & 0.00 & $\$ 17,038.03$ & 0.92 & 2 & 0.37 \\
\hline 53 & 54 & 209 & 33.49 & 31.58 & 34.93 & $\$ 18,568.96$ & 1.00 & 3 & 0.84 \\
\hline 54 & 55 & 4 & 25.00 & 25.00 & 50.00 & $\$ 19,456.75$ & 1.05 & 3 & 0.78 \\
\hline 55 & 56 & 1 & 0.00 & 0.00 & 100.00 & $\$ 22,317.68$ & 1.21 & 3 & 0.37 \\
\hline 56 & 57 & 10 & 90.00 & 10.00 & 0.00 & $\$ 15,534.57$ & 0.84 & 1 & 0.00 \\
\hline 57 & 58 & 25 & 32.00 & 56.00 & 12.00 & $\$ 17,600.83$ & 0.95 & 2 & 0.03 \\
\hline 58 & 59 & 17 & 0.00 & 17.65 & 82.35 & $\$ 21,542.04$ & 1.16 & 3 & 0.00 \\
\hline 59 & 60 & 40 & 2.50 & 15.00 & 82.50 & $\$ 21,482.18$ & 1.16 & 3 & 0.00 \\
\hline 60 & 61 & 21 & 52.38 & 33.33 & 14.29 & $\$ 17,160.56$ & 0.93 & 1 & 0.11 \\
\hline 61 & 62 & 9 & 55.56 & 33.33 & 11.11 & $\$ 16,936.78$ & 0.92 & 1 & 0.31 \\
\hline 62 & 63 & 168 & 1.19 & 26.79 & 72.02 & $\$ 21,056.48$ & 1.14 & 3 & 0.00 \\
\hline 63 & 64 & 2 & 0.00 & 0.00 & 100.00 & $\$ 22,317.68$ & 1.21 & 3 & 0.14 \\
\hline & & 4336 & 33.35 & 33.33 & 33.33 & $\$ 18,502.55$ & 1.00 & 1 & \\
\hline$\#$ & $\mathrm{~S}$ & freq & Tcb=1 & Tcb=2 & Tcb=3 & Exp. Value & Ratio & rule & $\mathrm{p}$ (margin) \\
\hline
\end{tabular}

The component Fit table for Nrb does not seem to show a strong effect for the Nrb component alone. In fact, the ratios for all of the bins of Nrb are close to the average for the entire sample and are within \pm 0.10 of 1 . So the effect size is small. However, because of the large frequencies of these three Nrb states, these effects are statistically significant, as the very small $p$-values indicate.

Table 78. Component Fit Table for IV Nrb (Knee) All IVs

Best Model: L Tcb : Svb Tcb : Nrb Tcb.

(Ratios between 0.91 and 1.09 are close to the margins and are indicated in gray.)

\begin{tabular}{|c|c|c|c|c|c|c|c|c|c|}
\hline & IV & \multicolumn{5}{|c|}{ Data } & & & \\
\hline & & & \multicolumn{3}{|c|}{ obs. $\mathrm{p}(\mathrm{DV} \mid \mathrm{IV})$} & & & \\
\hline$\#$ & Nrb & freq & Tcb=1 & Tcb=2 & Tcb=3 & Expected Value & Ratio & rule & $\mathrm{p}(\mathrm{margin})$ \\
\hline 1 & 1 & 1309 & 41.79 & 28.50 & 29.72 & 18119.85 & 0.98 & 1 & 0.00 \\
\hline 2 & 2 & 1474 & 37.31 & 36.09 & 26.59 & 18101.13 & 0.98 & 1 & 0.00 \\
\hline 3 & 3 & 1553 & 22.47 & 34.77 & 42.76 & 19205.40 & 1.04 & 3 & 0.00 \\
\hline & & 4336 & 33.35 & 33.33 & 33.33 & 18502.55 & 1.00 & 1 & \\
\hline
\end{tabular}

The Comorbidity IVs search resulted in the best model Ruh Tcb : Rmo Tcb :

Rde Tcb : Ros Tcb : Rhe Tcb that had a relatively small reduction in uncertainty of 
$1.5 \%$. The conditional probability distribution for this model does not provide for very meaningful predictions of expected total cost (Table 79). For the few rows that had a $\mathrm{p}($ margin $) \leq 0.05$ (rows $1,2,10,12$, and 18 ), the ratio is within \pm 0.10 of 1 , signifying that one would not expect a total cost much different than the average cost for the entire population. However, these small effects are statistically significant. These small effects could add up given high numbers of patients. While the ratio does indicate an important effect size, even small effects could have important financial impact given a large enough population of patients.

Table 79. Full Fit Table (Knee) with Comorbidity IVs for Best Model:

Ruh Tcb : Rmo Tcb : Rde Tcb : Ros Tcb : Rhe Tcb.

Blue rows indicate ratio $<0.90$ and orange rows indicate ratio $>1.10$ (ratios between 0.91 and 1.09 are close to the margins and are indicated in gray).

\begin{tabular}{|c|c|c|c|c|c|c|c|c|c|c|c|c|c|c|c|c|}
\hline \multicolumn{6}{|c|}{ IVs } & \multicolumn{4}{|l|}{ Data } & \multirow{2}{*}{\multicolumn{3}{|c|}{\begin{tabular}{|l} 
Model \\
calc $a($ DVIIV $)$
\end{tabular}}} & \multirow[b]{3}{*}{ Exp. Value } & \multirow[b]{3}{*}{ Ratio } & \multirow[b]{3}{*}{ rule } & \multirow[b]{3}{*}{$\mathrm{p}($ margin $)$} \\
\hline & & & & & & & \multicolumn{3}{|c|}{ obs. p(DV|IV) } & & & & & & & \\
\hline$\#$ & $\begin{array}{l}\mathrm{R} \\
\mathrm{u} \\
\mathrm{h}\end{array}$ & $\begin{array}{c}\mathrm{R} \\
\mathrm{m} \\
\mathrm{o}\end{array}$ & $\mid \begin{array}{c}\mathrm{R} \\
\mathrm{d} \\
\mathrm{e}\end{array}$ & $\begin{array}{l}\mathrm{R} \\
\mathrm{O} \\
\mathrm{s}\end{array}$ & $\begin{array}{l}R \\
h \\
e\end{array}$ & freq & $\mathrm{Tcb}=1$ & $\mathrm{Tcb}=2$ & $\mathrm{Tcb}=3$ & $\mathrm{Tcb}=1$ & $\mathrm{Tcb}=2$ & $T c b=3$ & & & & \\
\hline 1 & 0 & 0 & 0 & 0 & 0 & 1563 & 37.56 & 30.20 & 32.25 & 37.97 & 30.31 & 31.72 & $\$ 18,309.15$ & 0.99 & 1 & 0.00 \\
\hline 2 & 0 & 0 & 0 & 0 & 1 & 1559 & 36.37 & 36.24 & 27.39 & 36.17 & 36.27 & 27.57 & $\$ 18,174.41$ & 0.98 & 2 & 0.00 \\
\hline 3 & 0 & 0 & 0 & 1 & 0 & 37 & 24.32 & 21.62 & 54.05 & 27.99 & 23.76 & 48.25 & $\$ 19,300.57$ & 1.04 & 3 & 0.15 \\
\hline 4 & 0 & 0 & 0 & 1 & 1 & 76 & 31.58 & 27.63 & 40.79 & 27.48 & 29.31 & 43.22 & $\$ 19,092.76$ & 1.03 & 3 & 0.18 \\
\hline 5 & 0 & 0 & 1 & 0 & 0 & 134 & 28.36 & 31.34 & 40.30 & 27.21 & 30.33 & 42.46 & $\$ 19,066.79$ & 1.03 & 3 & 0.07 \\
\hline 6 & 0 & 0 & 1 & 0 & 1 & 170 & 27.06 & 35.88 & 37.06 & 26.15 & 36.62 & 37.23 & $\$ 18,865.04$ & 1.02 & 3 & 0.14 \\
\hline 7 & 0 & 0 & 1 & $1 \mid$ & 0 & 9 & 33.33 & 22.22 & 44.44 & 18.50 & 21.93 & 59.57 & $\$ 20,049.81$ & 1.08 & 3 & 0.29 \\
\hline 8 & 0 & 0 & 1 & 1 & 1 & 11 & 0.00 & 54.55 & 45.46 & 18.43 & 27.44 & 54.13 & $\$ 19,812.98$ & 1.07 & 3 & 0.32 \\
\hline 9 & 0 & 1 & 0 & 0 & 0 & 31 & 25.81 & 32.26 & 41.94 & 19.94 & 32.40 & 47.66 & $\$ 19,487.92$ & 1.05 & 3 & 0.18 \\
\hline 10 & 0 & 1 & 0 & 0 & 1 & 106 & 19.81 & 38.68 & 41.51 & 19.15 & 39.09 & 41.76 & $\$ 19,249.77$ & 1.04 & 3 & 0.01 \\
\hline 11 & 0 & 1 & 0 & 1 & 0 & 7 & 28.57 & 14.29 & 57.14 & 13.06 & 22.56 & 64.38 & $\$ 20,405.46$ & 1.10 & 3 & 0.26 \\
\hline 12 & 0 & 1 & 0 & 1 & 1 & 26 & 7.69 & 23.08 & 69.23 & 13.04 & 28.31 & 58.66 & $\$ 20,154.71$ & 1.09 & 3 & 0.02 \\
\hline 13 & 0 & 1 & 1 & 0 & 0 & 8 & 0.00 & 50.00 & 50.00 & 12.93 & 29.34 & 57.73 & $\$ 20,116.60$ & 1.09 & 3 & 0.29 \\
\hline 14 & 0 & 1 & 1 & \begin{tabular}{l|l}
0 \\
\end{tabular} & 1 & 25 & 4.00 & 40.00 & 56.00 & 12.62 & 35.97 & 51.40 & $\$ 19,846.72$ & 1.07 & 3 & 0.06 \\
\hline 15 & 0 & 1 & 1 & 1 & 0 & 4 & 25.00 & 0.00 & 75.00 & 7.92 & 19.12 & 72.96 & $\$ 20,918.99$ & 1.13 & 3 & 0.23 \\
\hline 16 & 0 & 1 & 1 & 1 & 1 & 6 & 0.00 & 50.00 & 50.00 & 8.04 & 24.38 & 67.58 & $\$ 20,679.17$ & 1.12 & 3 & 0.18 \\
\hline 17 & 1 & 0 & 0 & 0 & 0 & 128 & 31.25 & 32.81 & 35.94 & 27.82 & 30.94 & 41.24 & $\$ 18,996.71$ & 1.03 & 3 & 0.15 \\
\hline 18 & 1 & 0 & 0 & 0 & 1 & 293 & 24.57 & 38.91 & 36.52 & 26.67 & 37.26 & 36.07 & $\$ 18,800.37$ & 1.02 & 2 & 0.05 \\
\hline 19 & 1 & 0 & 0 & 1 & 0 & 4 & 50.00 & 50.00 & 0.00 & 19.08 & 22.57 & 58.35 & $\$ 19,981.08$ & 1.08 & 3 & 0.57 \\
\hline 20 & 1 & 0 & 0 & 1 & 1 & 29 & 13.79 & 27.59 & 58.62 & 18.95 & 28.17 & 52.89 & $\$ 19,744.07$ & 1.07 & 3 & 0.07 \\
\hline 21 & 1 & 0 & 1 & 0 & 0 & 24 & 4.17 & 25.00 & 70.83 & 18.79 & 29.18 & 52.03 & $\$ 19,710.74$ & 1.07 & 3 & 0.12 \\
\hline 22 & 1 & 0 & 1 & 0 & 1 & 38 & 31.58 & 21.05 & 47.37 & 18.26 & 35.62 & 46.12 & $\$ 19,465.14$ & 1.05 & 3 & 0.11 \\
\hline 23 & 1 & 0 & 1 & 1 & 0 & 3 & 0.00 & 0.00 & 100.00 & 11.95 & 19.75 & 68.30 & $\$ 20,607.26$ & 1.11 & 3 & 0.43 \\
\hline 24 & 1 & 0 & 1 & 1 & 1 & 8 & 12.50 & 25.00 & 62.50 & 12.07 & 25.04 & 62.90 & $\$ 20,366.80$ & 1.10 & 3 & 0.19 \\
\hline
\end{tabular}




\begin{tabular}{|c|c|c|c|c|c|c|c|c|c|c|c|c|c|c|c|}
\hline 25 & 1 & 0 & 0 & 0 & 8 & 25.00 & 25.00 & 50.00 & 13.32 & 30.17 & 56.51 & $\$ 20,052.45$ & 1.08 & 3 & 0.32 \\
\hline 26 & 1 & 1 & 0 & 1 & 17 & 17.65 & 35.29 & 47.06 & 12.96 & 36.88 & 50.16 & $\$ 19,783.03$ & 1.07 & 3 & 0.18 \\
\hline 27 & 1 & 10 & 1 & 0 & 2 & 0.00 & 0.00 & 100.00 & 8.23 & 19.81 & 71.97 & $\$ 20,867.33$ & 1.13 & 3 & 0.50 \\
\hline 28 & 1 & 1 & 1 & 1 & 2 & 0.00 & 0.00 & 100.00 & 8.33 & 25.20 & 66.48 & $\$ 20,623.37$ & 1.11 & 3 & 0.58 \\
\hline 29 & 1 & 1 & 0 & 0 & 1 & 0.00 & 0.00 & 100.00 & 8.28 & 26.17 & 65.56 & $\$ 20,584.28$ & 1.11 & 3 & 0.77 \\
\hline 30 & 1 & & 0 & 1 & 6 & 0.00 & 33.33 & 66.67 & 8.20 & 32.56 & 59.24 & $\$ 20,308.86$ & 1.10 & 3 & 0.31 \\
\hline 31 & 1 & 11 & 1 & 1 & 1 & 0.00 & 100.00 & 0.00 & 4.97 & 20.98 & 74.06 & $\$ 21,045.75$ & 1.14 & 3 & 0.68 \\
\hline & & & & & 4336 & 33.35 & 33.33 & 33.33 & 33.35 & 33.33 & 33.33 & $\$ 18,502.55$ & 1.00 & 1 & \\
\hline \# & $\mathrm{h}$ & & & $\begin{array}{l}\mathrm{R} \\
\mathrm{h} \\
\mathrm{e}\end{array}$ & freq & $\mathrm{Tcb}=1$ & $\mathrm{Tcb}=2$ & $\mathrm{Tcb}=3$ & $\mathrm{Tcb}=1$ & $\mathrm{Tcb}=2$ & $\mathrm{Tcb}=3$ & Exp. Value & Ratio & rule & $\mathrm{p}($ margin $)$ \\
\hline
\end{tabular}

The decision tree for the Comorbidities IVs fit table shows that when the individual comorbidities are all absent, or when only Rhe is present, the expected cost is less than the average (Figure 15). Notice that these risk ratios are all very close to 1 , however, and while significant, the difference is small.

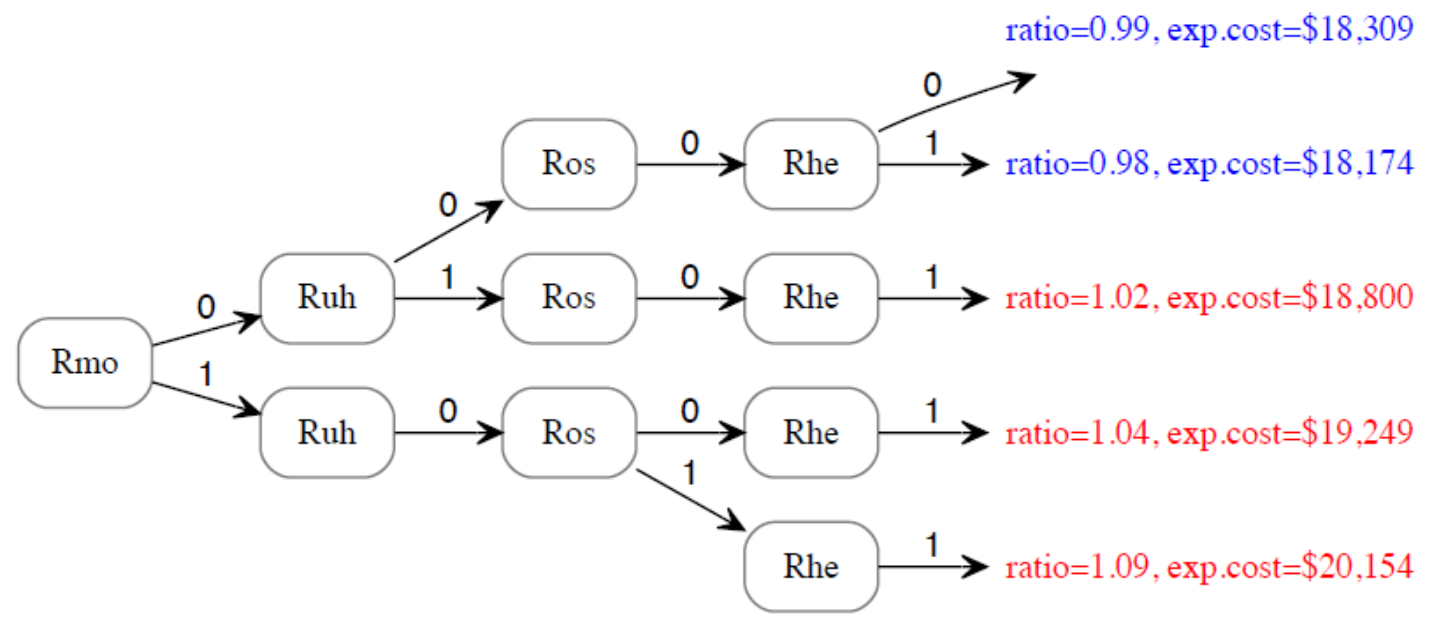

Figure 15. Decision Tree for DV Total Cost (Knee) with Comorbidity IVs for Best Model Ruh Tcb : Rmo Tcb : Rde Tcb : Ros Tcb : Rhe Tcb. 
Hip Analysis

- All IVs

○ Coarse Searches (Models without Loops)

In the final data set there were a total of 54 IVs retained after variable reduction

by a single predicting search. Of these IVs, four were found to be individually predictive with $\mathrm{p} \leq 0.05,17$ were from the literature and six of these 17 were in common with the single predicting variables from the search, while 11 IVs were found in the literature but did not have significant p-values in the single predicting search. The top 10 single predicting variables are shown in Table 80.

Table 80. Summary of Search Results (Hip) for All IVs for Total Cost Binned (Tcb). Search covers directed coarse, and fine models. All $p$ values $=0$.

\begin{tabular}{|c|c|c|c|c|}
\hline MODEL & $\Delta \mathrm{df}$ & $\triangle \mathrm{BIC}$ & $\% \Delta \mathrm{H}$ & Variable description \\
\hline \multicolumn{5}{|c|}{ COARSE, single predictors (top 10) } \\
\hline $\mathrm{S} \mathrm{Tcb}$ & 84 & 1026.62 & 24.2 & Surgeon \\
\hline $\mathrm{L} \mathrm{Tcb}$ & 12 & 849.389 & 13.4 & Location \\
\hline Svb Tcb & 4 & 893.509 & 13.1 & Surgeon volume binned \\
\hline $\mathrm{Da} \mathrm{Tcb}$ & 10 & 414.476 & 7.03 & Day of admit \\
\hline Ad Tcb & 76 & -333.31 & 3.98 & Admit diagnosis \\
\hline $\mathrm{Fc} \mathrm{Tcb}$ & 10 & -7.3465 & 1.04 & Financial class \\
\hline Nrb Tcb & 4 & 29.68 & 0.88 & Number of risks binned \\
\hline Rmo Tcb & 2 & 19.2819 & 0.5 & Morbid obesity (278.01) \\
\hline Ageb Tcb & 4 & 1.754 & 0.48 & Age binned \\
\hline Rrd Tcb & 2 & 14.5431 & 0.44 & Hypertensive renal disease (403.9) \\
\hline MODEL & $\Delta \mathrm{df}$ & $\triangle \mathrm{BIC}$ & $\% \Delta \mathrm{H}$ & Variable description \\
\hline \multicolumn{5}{|c|}{ COARSE, best model (loopless) } \\
\hline \multicolumn{5}{|c|}{$\Delta \mathrm{BIC}$} \\
\hline Svb Tcb & 40 & 1173.50 & 21.25 & Surgeon volume binned \\
\hline \multicolumn{5}{|c|}{ Inc.P \& $\triangle \mathrm{AIC}$ (same best model) } \\
\hline $\mathrm{S} \mathrm{Tcb}$ & 84 & 1026.62 & 24.21 & Surgeon \\
\hline MODEL & $\Delta \mathrm{df}$ & $\Delta \mathrm{BIC}$ & $\% \Delta \mathrm{H}$ & Variable description \\
\hline \multicolumn{5}{|c|}{ FINE, best models (with loops) } \\
\hline \multicolumn{5}{|c|}{$\Delta \mathrm{BIC}$ (best model) } \\
\hline $\begin{array}{l}\text { L Tcb : Svb Tcb: } \\
\text { Nrb Tcb }\end{array}$ & 20 & 1330.92 & 21.19 & $\begin{array}{l}\text { Location, Surgeon volume binned, Number of risks } \\
\text { binned }\end{array}$ \\
\hline \multicolumn{5}{|c|}{ Inc.P \& $\triangle \mathrm{AIC}$ (same best model) } \\
\hline S Tcb : Nrb Tcb & 88 & 1084.66 & 25.49 & Surgeon, Number of risks binned \\
\hline
\end{tabular}


Very similar to the knee analysis, the hip search results show that knowing the surgeon (S) reduces the uncertainty in predicting Total Cost Binned (Tcb) by $24.2 \%$ (Table 80), just a slight increase from the knee analysis. Location (L) is the next most individually predictive, with a $\% \Delta \mathrm{H}$ of 13.4 , followed by surgeon volume binned (Svb) with $\% \Delta \mathrm{H}$ at 13.1 . These are the same top three single predicting IVs as for the knee, but the $\% \Delta \mathrm{H}$ is quite a bit higher in the hip data. Financial class $(\mathrm{Fc})$ and age (Ageb) show up in the top 10 single predictors with $\% \Delta \mathrm{H}$ of 1.04 and 0.48 . The best coarse model that allows for more than one predicting component is L Svb Tcb (Table 80). Surgeon (S) is not in the best model selected by BIC, presumably because the complexity of the model $(\Delta \mathrm{df}=84)$ is not worth the information it adds. Apparently, surgeon volume $(\mathrm{Svb})$ along with location $(\mathrm{L})$ have enough information worth the complexity $(\Delta \mathrm{df}=40)$ with a $\% \Delta \mathrm{H}$ of 21. 25. The best coarse model allowing for more than one predicting variable (Table 80), L Svb Tcb $(\% \Delta H=21.25)$, has less than half the $\Delta$ df of single predicting model S Tcb $(\% \Delta H=24.2)$.

- Fine Searches (Models with Loops)

The fine-grained search did not detect an interaction effect, but found three single predicting components that together resulted in a $\% \Delta \mathrm{H}$ of 21.19 . This best fine-grained model by BIC, L Tcb : Svb Tcb : Nrb Tcb, has a slightly smaller $\% \Delta H$ than the best coarse model L Svb Tcb of $21.25 \%$, but the $\Delta$ df is exactly half with a $\Delta$ df of 20 rather than 40. This reduction in complexity more than compensates for the slightly smaller $\% \Delta \mathrm{H}$. 
- Comorbidity IVs

- Coarse Searches (Models without Loops)

The most predictive comorbidity in the Comorbidity IVs search is morbid obesity (Rmo), with a $\% \Delta \mathrm{H}$ of 0.50 (Table 81 ), identical to the most predictive single variable from the Comorbidity IVs search in the knee analysis (Table 73). The best coarse model that allows for more than one predicting variable adds hypertensive renal disease (Rrd), resulting in the best coarse model Rmo Rrd Tcb with a $\% \Delta \mathrm{H}$ of 0.97 and a $\Delta$ df of 6 (Table 81). These are very small reductions of uncertainty. 
Table 81. Summary of Search Results (Hip) for Comorbidity IVs for DV Total Cost Binned (Tcb). Search covers coarse and fine models. All $p$ values $=0$.

\begin{tabular}{|c|c|c|c|c|}
\hline MODEL & $\Delta \mathrm{df}$ & $\Delta \mathrm{BIC}$ & $\% \Delta \mathrm{H}$ & Variable description \\
\hline \multicolumn{5}{|c|}{ COARSE, single predictors } \\
\hline Rmo Tcb & 2 & 19.28 & 0.50 & Morbid obesity (278.01) \\
\hline Rrd Tcb & 2 & 14.54 & 0.44 & Hypertensive renal disease (403.9) \\
\hline Rkd Tcb & 2 & 9.23 & 0.36 & Chronic kidney disease, Stage III (585.3) \\
\hline Rnr Tcb & 2 & 5.08 & 0.30 & Aseptic necrosis of head and neck of femur (733.42) \\
\hline Rcp Tcb & 2 & 4.17 & 0.29 & Chronic pain (338.29) \\
\hline Raf Tcb & 2 & 1.92 & 0.26 & Atrial fibrillation (427.31) \\
\hline Rdi Tcb & 2 & 1.06 & 0.24 & Diabetes mellitus (250) \\
\hline Rog Tcb & 2 & -0.26 & 0.23 & Osteoarthrosis (715.96) \\
\hline Rku Tcb & 2 & -1.55 & 0.21 & Chronic kidney disease (585.9) \\
\hline Rin Tcb & 2 & -2.52 & 0.19 & Urinary incontinence (788.3) \\
\hline MODEL & $\Delta \mathrm{df}$ & $\Delta \mathrm{BIC}$ & $\% \Delta \mathrm{H}$ & Variable description \\
\hline \multicolumn{5}{|c|}{ COARSE, IVs in AIC or BIC models but not in top 10} \\
\hline $\operatorname{Rcg}(\operatorname{rank} 23)$ & 2 & -5.14 & 0.16 & Other disorders of bone and cartilage (733.99) \\
\hline MODEL & $\Delta \mathrm{df}$ & $\triangle \mathrm{BIC}$ & $\% \Delta \mathrm{H}$ & Variable description \\
\hline \multicolumn{5}{|c|}{ COARSE, best model (loopless) } \\
\hline \multicolumn{5}{|c|}{$\Delta \mathrm{BIC}$ (best model) } \\
\hline RmoRrd Tcb & 6 & 19.91 & 0.97 & $\begin{array}{l}\text { Morbid obesity (278.01) + Hypertensive renal disease } \\
(403.9)\end{array}$ \\
\hline \multicolumn{5}{|l|}{$\Delta \mathrm{AIC}$ (best model) } \\
\hline RmoRrdRnr Tcb & 14 & -18.73 & 1.34 & $\begin{array}{l}\text { Morbid obesity (278.01) + Hypertensive renal disease } \\
(403.9)+\text { Aseptic necrosis of head and neck of femur } \\
(733.42)\end{array}$ \\
\hline \multicolumn{5}{|l|}{ Inc.P (best model) } \\
\hline RmoRcpRrdRnr Tcb & 30 & -121.70 & 1.71 & $\begin{array}{l}\text { Morbid obesity (278.01) + Chronic pain (338.29) + } \\
\text { Hypertensive renal disease }(403.9)+\text { Aseptic necrosis of } \\
\text { head and neck of femur }(733.42)\end{array}$ \\
\hline MODEL & $\Delta \mathrm{df}$ & $\Delta \mathrm{BIC}$ & $\% \Delta \mathrm{H}$ & Variable description \\
\hline \multicolumn{5}{|c|}{ FINE, best models (with loops) } \\
\hline \multicolumn{5}{|l|}{$\triangle \mathrm{BIC}$ (best model) } \\
\hline $\begin{array}{l}\text { Rmo Tcb : Rcp Tcb : } \\
\text { Rrd Tcb : Rnr Tcb }\end{array}$ & 8 & 41.95 & 1.51 & $\begin{array}{l}\text { Morbid obesity (278.01), Chronic pain (338.29), } \\
\text { Hypertensive renal disease (403.9), Aseptic necrosis of } \\
\text { head and neck of femur (733.42) }\end{array}$ \\
\hline \multicolumn{5}{|c|}{ Inc.P \& $\triangle$ AIC (same best model) } \\
\hline $\begin{array}{l}\text { Rmo Tcb : Rcp Tcb : } \\
\text { Rrd Tcb : Raf Tcb : } \\
\text { Rog Tcb : Rnr Tcb : } \\
\text { Rcg Tcb }\end{array}$ & 14 & 33.79 & 2.08 & $\begin{array}{l}\text { Morbid obesity (278.01), Chronic pain (338.29), } \\
\text { Hypertensive renal disease (403.9), Atrial fibrillation } \\
\text { (427.31), Osteoarthrosis (715.96), Aseptic necrosis of } \\
\text { head and neck of femur (733.42), Other disorders of bone } \\
\text { and cartilage (733.99) }\end{array}$ \\
\hline
\end{tabular}

\section{- Fine Searches (Models with Loops)}

The best model by BIC for Comorbidity IVs is Rmo Tcb : Rcp Tcb : Rrd Tcb :

Rnr Tcb, with a \% $\Delta \mathrm{H}$ of $1.51(\Delta \mathrm{df}=8)$. Morbid obesity (Rmo), chronic pain (Rcp), 
hypertensive renal disease (Rrd), and aseptic necrosis of head and neck of femur (Rnr) are looked at in detail in the model's conditional probability distribution, given below.

\section{- Comparing Search Results (Hip, Tcb)}

The All IVs and the Comorbidity IVs searches yield two sets of results that can be compared to each other and interpreted with a three-tiered classification of results, as described below and summarized in Table 82 .

Table 82. The 3-Tiered Classification of Predicting Variables for DV: Total Cost, Hip Analysis

\begin{tabular}{|l|l|}
\hline Tier & Variables \\
\hline Tier 1 - Most Important & L, Svb, Nrb \\
\hline Tier 2 & None \\
\hline Tier 3 & S, Rmo, Rcp, Rrd, Rnr \\
\hline
\end{tabular}

Tier 1 contains variables from the best-by-BIC model from the dataset All IVs. This is the most complete search, and the one that provides the most conservative predictors. The selected variables ( $\mathrm{L}, \mathrm{Svb}$, and $\mathrm{Nrb}$ ) are shown in the first row of Table 82, and are considered the most important predicting variables.

Tier 2 contains variables not in Tier1, but found in the AIC (less conservative) model of All IVs AND in the BIC model of Comorbidity IVs. For the Hip Analysis of DV Total Cost, there are no variables remaining.

Finally, this classification places into Tier 3 any variables present elsewhere in the best model search results, but not included in Tier 1 or Tier 21 that is, any variables unique to one of the two searches - variables in the AIC model of All IVs but not in the BIC model of Comorbidity IVs, and variables in the BIC model of Comorbidity IVs but 
not in the AIC model from All IVs. This shows the next most important predicting variables (S, Rmo, Rcp, Rrd, and Rnr) in the last row of Table 82.

\section{- Model FIT}

The detailed joint conditional probability distribution for best model L Tcb : Svb Tcb : Nrb Tcb from the All IVs search is shown in Table 84. Total Cost was binned into 3 bins, with the ranges, frequencies, and average costs shown in Table 83.

Table 83. Total Cost Range, Average Cost, and Frequency.

\begin{tabular}{|c|c|c|c|c|}
\hline Bin & Min Cost & Max Cost & Average Cost & Frequency \\
\hline 1 & $\$ 11,147$ & $\$ 16,768$ & $\$ 15,244$ & 1068 \\
\hline 2 & $\$ 16,772$ & $\$ 19,192$ & $\$ 17,997$ & 1069 \\
\hline 3 & $\$ 19,195$ & $\$ 71,264$ & $\$ 22,534$ & 1068 \\
\hline
\end{tabular}


Table 84. Full Fit Table (Hip) with All IVs for Best Model: L Tcb : Svb Tcb : Nrb Tcb.

Blue rows indicate ratio $<0.90$ and orange rows indicate ratio $>1.10$.

(Ratios between 0.91 and 1.09 are close to the margins and are indicated in gray.)

\begin{tabular}{|c|c|c|c|c|c|c|c|c|c|c|c|c|c|c|}
\hline \multicolumn{4}{|c|}{ IVs } & \multicolumn{4}{|l|}{ Data } & \multicolumn{3}{|c|}{ Model } & \multirow[b]{3}{*}{ Exp. Value } & \multirow[b]{3}{*}{ Ratio } & \multirow[b]{3}{*}{ rule } & \multirow[b]{3}{*}{$\mathrm{p}($ margin $)$} \\
\hline & & & & & \multicolumn{3}{|c|}{ obs. p(DV|IV) } & calc & c. $q(D V$ & IV) & & & & \\
\hline$\#$ & $\mathrm{~L}$ & Svb & $\mathrm{Nrb}$ & freq & $\mathrm{Tcb}=1$ & $\mathrm{Tcb}=2$ & $\mathrm{Tcb}=3$ & $\mathrm{Tcb}=1$ & $\mathrm{Tcb}=2$ & $\mathrm{Tcb}=3$ & & & & \\
\hline 1 & 1 & 1 & 1 & 21 & 0.00 & 23.81 & 76.19 & 2.05 & 23.53 & 74.42 & $\$ 21,317.04$ & 1.15 & 3 & 0.00 \\
\hline 2 & 1 & 2 & 1 & 81 & 2.47 & 25.93 & 71.61 & 1.94 & 26.00 & 72.06 & $\$ 21,213.25$ & 1.14 & 3 & 0.00 \\
\hline 3 & 2 & 1 & 1 & 215 & 12.56 & 48.84 & 38.61 & 11.01 & 50.34 & 38.65 & $19,447.72$ & 1.05 & 2 & \\
\hline 4 & 2 & 1 & 2 & 214 & 4.21 & 44.39 & 51.40 & 7.51 & 45.10 & 47.39 & $19,940.50$ & 1.07 & 3 & \\
\hline 5 & 2 & 1 & 3 & 198 & 3.54 & 35.35 & 61.11 & 4.13 & 37.68 & 58.19 & $20,523.33$ & 1.10 & 3 & \\
\hline 6 & 2 & 2 & 1 & 172 & 9.30 & 59.88 & 30.81 & 10.05 & 53.77 & 36.18 & $19,362.02$ & 1.04 & 2 & \\
\hline 7 & 2 & 2 & 2 & 193 & 7.25 & 50.78 & 41.97 & 6.90 & 48.48 & 44.63 & $\$ 19,832.19$ & 1.07 & 2 & \\
\hline 8 & 2 & 2 & 3 & 168 & 7.14 & 37.50 & 55.36 & 3.83 & 40.87 & 55.31 & & 1.10 & 3 & \\
\hline 9 & 3 & 1 & 1 & 52 & 11.54 & 40.39 & 48.08 & 21.05 & 37.29 & 41.66 & $19,307.42$ & 1.04 & 3 & \\
\hline 10 & 3 & 1 & 2 & 77 & 12.99 & 31.17 & 55.84 & 14.52 & 33.81 & 51.67 & 941.54 & 1.07 & 3 & \\
\hline 11 & 3 & 1 & 3 & 125 & 4.80 & 32.00 & 63.20 & 8.02 & 28.33 & 63.65 & 664.32 & 1.11 & 3 & \\
\hline 12 & 3 & 2 & 1 & 36 & 8.33 & 30.56 & 61.11 & 19.60 & 40.63 & 39.77 & & 1.04 & 2 & \\
\hline 13 & 3 & 2 & 2 & 48 & 10.42 & 27.08 & 62.50 & 13.57 & 36.95 & 49.49 & 88.89 & 1.07 & 3 & \\
\hline 14 & 3 & 2 & 3 & 51 & 7.84 & 29.41 & 62.75 & 7.53 & 31.15 & 61.32 & 571.98 & 1.11 & 3 & \\
\hline 15 & 3 & 3 & 1 & 393 & 77.10 & 19.08 & 3.82 & 74.65 & 18.86 & & 5.60 & $0 . \varepsilon$ & 1 & \\
\hline 16 & 3 & 3 & 2 & 396 & 68.94 & 23.23 & 7.83 & 67.20 & 22.31 & 10.49 & 22.80 & 0.89 & 1 & 0.0 \\
\hline 17 & 3 & 3 & 3 & 354 & 53.67 & 27.40 & 18.93 & 53.97 & 27.22 & 18.81 & 64.37 & 0.93 & 1 & 0.0 \\
\hline 18 & 4 & 1 & 1 & 13 & 46.15 & 30.77 & 23.08 & 5.24 & 40.73 & 54.03 & 04.03 & 1.09 & 3 & 0.1 \\
\hline 19 & 4 & 1 & 2 & 19 & 42.11 & 42.11 & 15.79 & 3.36 & 34.33 & 62.31 & 731.58 & 1.12 & 3 & 0.01 \\
\hline 20 & 4 & 1 & 3 & 15 & 20.00 & 60.00 & 20.00 & 1.73 & 26.80 & 71.48 & 92.62 & 1.14 & 3 & 0.00 \\
\hline 21 & 4 & 3 & 1 & 42 & 19.05 & 33.33 & 47.62 & 39.05 & 43.29 & 17.66 &, 723.28 & 0.95 & 2 & 0.09 \\
\hline 22 & 4 & 3 & 2 & 67 & 22.39 & 43.28 & 34.33 & 30.58 & 44.55 & 24.87 & $\$ 18,283.50$ & 0.98 & 2 & 0.1 \\
\hline 23 & 4 & 3 & 3 & 48 & 16.67 & 43.75 & 39.58 & 19.89 & 44.01 & 36.11 & $\$ 19,087.69$ & 1.03 & 2 & 0.1 \\
\hline 24 & 5 & 3 & 1 & 27 & 51.85 & 29.63 & 18.52 & 53.73 & 32.70 & 13.58 & $\$ 17,133.84$ & 0.92 & 1 & 0.04 \\
\hline 25 & 5 & 3 & 2 & 30 & 43.33 & 36.67 & 20.00 & 44.36 & 35.48 & 20.16 & $\$ 17,690.49$ & 0.95 & 1 & 0.26 \\
\hline 26 & 5 & 3 & 3 & 20 & 35.00 & 40.00 & 25.00 & 30.97 & 37.62 & 31.42 & $\$ 18,569.76$ & 1.00 & 2 & 0.92 \\
\hline 27 & 6 & 1 & 1 & 47 & 91.49 & 6.38 & 2.13 & 89.53 & 6.01 & 4.46 & $\$ 15,734.15$ & 0.85 & 1 & 0.00 \\
\hline 28 & 6 & 1 & 2 & 37 & 86.49 & 5.41 & 8.11 & 84.91 & 7.49 & 7.61 & $\$ 16,004.23$ & 0.86 & 1 & 0.00 \\
\hline 29 & 6 & 1 & 3 & 34 & 70.59 & 11.77 & 17.65 & 74.97 & 10.04 & 14.99 & $\$ 16,612.93$ & 0.89 & 1 & 0.00 \\
\hline 30 & 7 & 2 & 1 & 12 & 0.00 & 0.00 & 100.00 & 0.00 & 0.00 & 100.00 & $\$ 22,533.93$ & 1.21 & 3 & 0.00 \\
\hline & & & & 3205 & 33.32 & 33.35 & 33.32 & 33.32 & 33.35 & 33.32 & $\$ 18,591.45$ & 1.00 & 2 & \\
\hline$\#$ & $\mathrm{~L}$ & Svb & $\mathrm{Nrb}$ & freq & $\mathrm{Tcb}=1$ & $\mathrm{Tcb}=2$ & $\mathrm{Tcb}=3$ & $\mathrm{Tcb}=1$ & $T c b=2$ & $\mathrm{Tcb}=3$ & Exp. Value & Ratio & rule & $\mathrm{p}($ margin $)$ \\
\hline
\end{tabular}

Location 1 with a lower surgeon volume (Svb) and patients with low number of

risks (Nrb 1 or 2) has high expected costs (rows 1 and 2) as seen in Table 84. For

location $=2$, regardless of the state of $\mathrm{Svb}$ or $\mathrm{Nrb}$, it is consistently close to the observed

average total cost in the data, and even though $p<0.05$ criteria, the ratio is within \pm 0.10 .

For location 3, when $\mathrm{Svb}$ is low (bin 1 or 2), and $\mathrm{Nrb}$ is 2 or 3, the costs are expected to 
be higher (rows 11 and 14). For that same location, when Svb is high (bin 3), Nrb is lower in bins 1 and 2, and the costs are expected to be lower (rows 15 and 16). In any of the rows for location 4 where $\mathrm{p} \leq 0.05$, the surgeon volume (Svb) is low (bin 1$)$ and number of risks higher (bin 1 and 2) each time, resulting in expected higher costs. Rows 27-29 show that location 6, with Svb low in bin 1 and regardless of Nrb, always has lower expected costs (Table 84).

The individual component table for location (L) in Table 85 below show that location $=4$ alone does not predict expected cost much higher than the average (ratio 1.02). However, when combined with surgeon volume (Svb) and number of risks $(\mathrm{Nrb})$ in the joint distribution (Table 84$)$, location $=4$ has higher ratios $(1.12$ and 1.14 in rows 19 and 20, respectively).

Table 85. Component Fit Table for IV L (Hip) All IVs Best Model: L Tcb : Svb Tcb : Nrb Tcb. Blue rows indicate ratio $<0.90$ and orange rows indicate ratio $>1.10$.

(Ratios between 0.91 and 1.09 are close to the margins and are indicated in gray.)

\begin{tabular}{|c|c|c|c|c|c|c|c|c|c|}
\hline & IV & Data & \multicolumn{3}{|c|}{ obs. $\mathrm{p}(\mathrm{DV} \mid \mathrm{IV})$} & & & \\
\hline$\#$ & $\mathrm{~L}$ & freq & $\mathrm{Tcb}=1$ & $\mathrm{Tcb}=2$ & $\mathrm{Tcb}=3$ & Expected Value & Ratio & rule & $\mathrm{p}(\mathrm{margin})$ \\
\hline 1 & 1 & 102 & 1.96 & 25.49 & 72.55 & $\$ 21,234.61$ & 1.14 & 3 & 0.00 \\
\hline 2 & 2 & 1160 & 7.33 & 46.03 & 46.64 & $\$ 19,911.36$ & 1.07 & 3 & 0.00 \\
\hline 3 & 3 & 1532 & 52.22 & 25.33 & 22.45 & $\$ 17,577.82$ & 0.95 & 1 & 0.00 \\
\hline 4 & 4 & 204 & 23.53 & 41.67 & 34.80 & $\$ 18,928.35$ & 1.02 & 2 & 0.01 \\
\hline 5 & 5 & 77 & 44.16 & 35.07 & 20.78 & $\$ 17,724.06$ & 0.95 & 1 & 0.04 \\
\hline 6 & 6 & 118 & 83.90 & 7.63 & 8.48 & $\$ 16,071.44$ & 0.86 & 1 & 0.00 \\
\hline 7 & 7 & 12 & 0.00 & 0.00 & 100.00 & $\$ 22,533.93$ & 1.21 & 3 & 0.00 \\
\hline & & 3205 & 33.32 & 33.35 & 33.32 & $\$ 18,591.45$ & 1.00 & 2 & \\
\hline
\end{tabular}

Table 86. Component Fit Table for IV Svb (Hip) All IVs Best Model: L Tcb : Svb Tcb : Nrb Tcb. (Ratios between 0.91 and 1.09 are close to the margins and are indicated in gray.)

\begin{tabular}{|c|c|c|c|c|c|c|c|c|c|}
\hline & IV & Data & \multicolumn{3}{|c|}{ obs. $\mathrm{p}(\mathrm{DV} \mid \mathrm{IV})$} & & & & \\
\hline$\#$ & Svb & freq & Tcb=1 & Tcb=2 & Tcb=3 & Expected Value & Ratio & rule & $\mathrm{p}(\mathrm{margin})$ \\
\hline 1 & 1 & 1067 & 16.96 & 36.55 & 46.49 & $\$ 19,638.90$ & 1.06 & 3 & 0.00 \\
\hline 2 & 2 & 761 & 7.36 & 42.58 & 50.07 & $\$ 20,066.20$ & 1.08 & 3 & 0.00 \\
\hline 3 & 3 & 1377 & 60.35 & 25.78 & 13.87 & $\$ 16,964.92$ & 0.91 & 1 & 0.00 \\
\hline & & 3205 & 33.32 & 33.35 & 33.32 & $\$ 18,591.45$ & 1.00 & 2 & \\
\hline
\end{tabular}


Table 87. Component Fit Table for IV Nrb (Hip) All IVs Best Model: L Tcb : Svb Tcb : Nrb Tcb.

(Ratios between 0.91 and 1.09 are close to the margins and are indicated in gray.)

\begin{tabular}{|c|c|c|c|c|c|c|c|c|c|}
\hline & IV & Data & \multicolumn{3}{|c|}{ obs. $\mathrm{p}(\mathrm{DV} \mid \mathrm{IV})$} & & & \\
\hline$\#$ & Nrb & freq & Tcb=1 & Tcb=2 & Tcb=3 & Expected Value & Ratio & rule & $\mathrm{p}$ (margin) \\
\hline 1 & 1 & 1111 & 38.52 & 33.30 & 28.17 & $\$ 18,214.59$ & 0.98 & 1 & 0.00 \\
\hline 2 & 2 & 1081 & 35.06 & 34.41 & 30.53 & $\$ 18,416.77$ & 0.99 & 1 & 0.14 \\
\hline 3 & 3 & 1013 & 25.77 & 32.28 & 41.96 & $\$ 19,191.18$ & 1.03 & 3 & 0.00 \\
\hline & & 3205 & 33.32 & 33.35 & 33.32 & $\$ 18,591.45$ & 1.00 & 2 & \\
\hline
\end{tabular}

The Comorbidity IVs search resulted in the best BIC model Rmo Tcb : Rcp Tcb :

Rrd Tcb : Rnr Tcb, whose conditional probability distribution is shown in Table 88.

Row 11 shows that when morbid obesity (Rmo) and chronic pain (Rcp) are both present

$($ freq $=11)$, the expected cost is $\$ 20,841$, significantly higher than the margin of $\$ 18,591$.

Table 88. Full Fit Table (Hip) with Comorbidity IVs for

Best Model: Rmo Tcb : Rcp Tcb : Rrd Tcb : Rnr Tcb.

Blue rows indicate ratio $<0.90$ and orange rows indicate ratio $>1.10$.

(Ratios between 0.91 and 1.09 are close to the margins and are indicated in gray.)

\begin{tabular}{|c|c|c|c|c|c|c|c|c|c|c|c|c|c|c|c|}
\hline & \multicolumn{4}{|c|}{ IVs } & \multicolumn{4}{|c|}{ Data } & \multirow{2}{*}{\multicolumn{3}{|c|}{ Model }} & \multirow[b]{3}{*}{ Exp. Value } & \multirow[b]{3}{*}{ Ratio } & \multirow[b]{3}{*}{ rule } & \multirow[b]{3}{*}{$\mathrm{p}($ margin $)$} \\
\hline & & & & & & \multicolumn{3}{|c|}{ obs. p(DV|IV) } & & & & & & & \\
\hline \# & $\begin{array}{c}\mathrm{R} \\
\mathrm{m} \\
\mathrm{O}\end{array}$ & $\begin{array}{l}\mathrm{R} \\
\mathrm{c} \\
\mathrm{p}\end{array}$ & $\begin{array}{c}\mathrm{R} \\
\mathrm{r} \\
\mathrm{d}\end{array}$ & $\begin{array}{l}\mathrm{R} \\
\mathrm{n} \\
\mathrm{r}\end{array}$ & freq & $\mathrm{Tcb}=1$ & $\mathrm{Tcb}=2$ & $\mathrm{Tcb}=3$ & $\mathrm{Tcb}=1$ & $\mathrm{Tcb}=2$ & $\mathrm{Tcb}=3$ & & & & \\
\hline 1 & 0 & 0 & 0 & 0 & 2797 & 35.65 & 33.82 & 30.53 & 35.73 & 33.83 & 30.44 & $\$ 18,394.66$ & 0.99 & 1 & 0.00 \\
\hline 2 & 0 & 0 & 0 & 1 & 88 & 20.46 & 30.68 & 48.86 & 19.36 & 28.12 & 52.53 & $\$ 19,847.44$ & 1.07 & 3 & 0.00 \\
\hline 3 & 0 & 0 & 1 & 0 & 69 & 11.59 & 31.88 & 56.52 & 10.96 & 30.99 & 58.05 & $\$ 20,329.12$ & 1.09 & 3 & 0.00 \\
\hline 4 & 0 & 0 & 1 & 1 & 1 & 0.00 & 0.00 & 100.00 & 4.50 & 19.53 & 75.97 & $\$ 21,319.72$ & 1.15 & 3 & 0.65 \\
\hline 5 & 0 & 1 & 0 & 0 & 94 & 26.60 & 25.53 & 47.87 & 24.51 & 26.72 & 48.78 & $\$ 19,535.15$ & 1.05 & 3 & 0.01 \\
\hline 6 & 0 & 1 & 0 & 1 & 6 & 0.00 & 0.00 & 100.00 & 11.10 & 18.56 & 70.35 & $\$ 20,883.27$ & 1.12 & 3 & 0.15 \\
\hline 7 & 0 & 1 & 1 & 0 & 6 & 0.00 & 16.67 & 83.33 & 6.01 & 19.58 & 74.41 & $\$ 21,207.25$ & 1.14 & 3 & 0.09 \\
\hline 8 & 1 & 0 & 0 & 0 & 128 & 15.63 & 34.38 & 50.00 & 14.67 & 35.32 & 50.01 & $\$ 19,862.43$ & 1.07 & 3 & 0.00 \\
\hline 9 & 1 & 0 & 0 & 1 & 4 & 0.00 & 0.00 & 100.00 & 6.43 & 23.75 & 69.83 & $\$ 20,988.02$ & 1.13 & 3 & 0.28 \\
\hline 10 & 1 & 0 & 1 & 0 & 1 & 0.00 & 0.00 & 100.00 & 3.40 & 24.47 & 72.13 & $\$ 21,175.72$ & 1.14 & 3 & 0.69 \\
\hline 11 & 1 & 1 & 0 & 0 & 11 & 0.00 & 45.46 & 54.55 & 8.52 & 23.62 & 67.86 & $\$ 20,841.43$ & 1.12 & 3 & 0.04 \\
\hline & & & & & 3205 & 33.32 & 33.35 & 33.32 & 33.32 & 33.35 & 33.32 & $\$ 18,591.45$ & 1.00 & 2 & \\
\hline
\end{tabular}

The decision tree for model Rmo Tcb : Rcp Tcb : Rrd Tcb : Rnr Tcb (Figure 16) shows that when Rmo and Rcp are both present, the ratio for expected cost is 1.12. 
Table 89. Component Fit Table (Hip) for morbid obesity (Rmo) with Comorbidity IVs for Best Model: Rmo Tcb : Rcp Tcb : Rrd Tcb : Rnr Tcb.

Blue rows indicate ratio $<0.90$ and orange rows indicate ratio $>1.10$.

(Ratios between 0.91 and 1.09 are close to the margins and are indicated in gray.)

\begin{tabular}{|c|c|c|c|c|c|c|c|c|c|}
\hline & IV & Data & \multicolumn{3}{|c|}{ obs. $\mathrm{p}(\mathrm{DV} \mid \mathrm{IV})$} & & & \\
\hline$\#$ & Rmo & freq & Tcb=1 & Tcb=2 & Tcb=3 & Expected Value & Ratio & rule & $\mathrm{p}(\mathrm{margin})$ \\
\hline 1 & 0 & 3061 & 34.24 & 33.32 & 32.44 & $\$ 18,526.04$ & 1.00 & 1 & 0.48 \\
\hline 2 & 1 & 144 & 13.89 & 34.03 & 52.08 & $\$ 19,977.69$ & 1.07 & 3 & 0.00 \\
\hline & & 3205 & 33.32 & 33.35 & 33.32 & $\$ 18,591.45$ & 1.00 & 2 & \\
\hline
\end{tabular}

Table 90. Component Fit Table (Hip) for chronic pain (Rcp) with Comorbidity IVs for Best Model: Rmo Tcb : Rcp Tcb : Rrd Tcb : Rnr Tcb.

Blue rows indicate ratio $<0.90$ and orange rows indicate ratio $>1.10$.

(Ratios between 0.91 and 1.09 are close to the margins and are indicated in gray.)

\begin{tabular}{|c|c|c|c|c|c|c|c|c|c|}
\hline & IV & Data & obs. p(D) & IV) & & & & & \\
\hline$\#$ & Rcp & freq & $\mathrm{Tcb}=1$ & $\mathrm{Tcb}=2$ & $\mathrm{Tcb}=3$ & Expected Value & Ratio & rule & $\mathrm{p}($ margin $)$ \\
\hline 1 & 0 & 3088 & 33.78 & 33.65 & 32.58 & $\$ 18,545.18$ & 1.00 & 1 & 0.68 \\
\hline 2 & 1 & 117 & 21.37 & 25.64 & 52.99 & $\$ 19,812.92$ & 1.07 & 3 & 0.00 \\
\hline & & 3205 & 33.32 & 33.35 & 33.32 & $\$ 18,591.45$ & 1.00 & 2 & \\
\hline
\end{tabular}

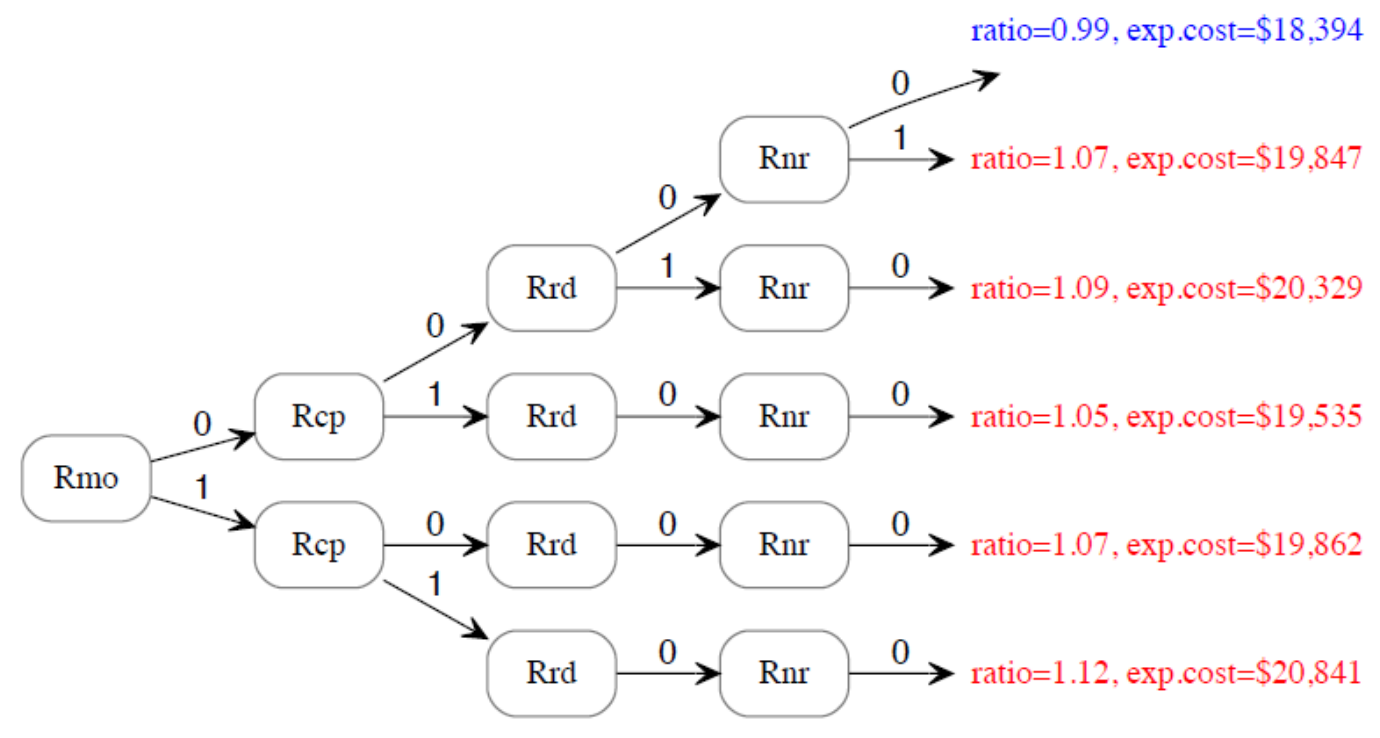

Figure 16. Decision Tree for DV Total Cost (Hip) with Comorbidity IVs for Best Model Rmo Tcb : Rcp Tcb : Rrd Tcb : Rnr Tcb. 
Hip \& Knee Summary for Total Cost

The most important IVs across both the hip and knee searches for Total Cost (Tcb) are summarized in Table 91. The IVs in common are surgeon (S), number of risks (Nrb) and morbid obesity (Rmo).

Table 91. Summary of Most Important IVs by Tier Across Hip and Knee for Total Cost.

\begin{tabular}{|c|l|l|}
\hline & \multicolumn{2}{|c|}{ Total Cost (Tcb) } \\
\hline Tier & Knee & Hip \\
\hline 1 & S, Nrb & L, Svb, Nrb \\
\hline 2 & Rmo & None \\
\hline 3 & Rmn, Rug, Rht, Rnn, Ruh, Rde, Ros, Rhe & S, Rmo, Rcp, Rrd, Rnr \\
\hline
\end{tabular}

In this chapter, LR and RA were compared using both confirmatory and exploratory modes. Then, RA was used for exploratory modeling, resulting in 16 predictive models. These best predictors were determined, and the models themselves were analyzed in detail. The Results chapter looked at each DV separately. In the next chapter, Discussion, summaries are provided that look across the DVs. After summarizing predictors and risk ratios within and between DVs, conclusions are offered that aim to relay a few key insights resulting from this project. 


\section{Chapter 5. Discussion}

This project applied Reconstructability Analysis (RA) to outcomes in hip and knee replacement surgery, and asked if RA could create useful models of outcomes, and whether these models could produce predictions complimentary to - and even stronger than-models produced by LR. This inquiry resulted in substantive findings of interest to clinical and healthcare administrative professionals as well as to researchers and data scientists interested in improving the methodological toolkit for predictive analytics.

The discussion that follows summarizes the contributions of the project in the terms and interests of these audiences. The first section, organized around a healthcare perspective, includes (a) identifying important predictors of outcome variables,

(b) distinguishing between patient-centered and delivery system-centered predictors, and (c) classifying patients as being of higher or lower risk for adverse outcomes. The second section, organized toward a methodological perspective, includes (a) relating RA, the primary methodology used in this project, to the more commonly used approach of logistic regression, and (b) augmenting the use of RA for the analysis of this type of biomedical data. Finally, additional discussion that addresses future research and applications is provided. 


\section{Clinical and Healthcare-Related Contributions}

\section{Best Models}

A primary purpose of this project was to perform RA exploratory analysis and 16 best models predictive of each of the DVs. The amount of uncertainty reduced $(\% \Delta H)$ is the primary measure indicating prediction (similar to \%variance). The 16 models are listed below in Table 92 . These models are sorted by $\% \Delta \mathrm{H}$, with the most predictive models at the top. The IV descriptions are provided with novel IVs indicated in blue and any interaction terms underlined.

Table 92. List of 16 Models sorted by $\% \Delta H$.

\begin{tabular}{|c|c|c|c|}
\hline Data Set & MODEL & $\% \Delta H$ & Variable description \\
\hline $\begin{array}{l}\text { Knee, All } \\
\text { IVs }\end{array}$ & S Tcb : Nrb Tcb & 25.13 & Surgeon, Number of Risks Binned \\
\hline $\begin{array}{l}\text { Hip, All } \\
\text { IVs }\end{array}$ & $\begin{array}{l}\text { Fc SNF : Ageb SNF : } \\
\text { Svb SNF :Nrb SNF }\end{array}$ & 23.61 & $\begin{array}{l}\text { Financial class, Age binned, Surgeon volume binned, } \\
\text { Number of risks binned }\end{array}$ \\
\hline $\begin{array}{l}\text { Hip, All } \\
\text { IVs }\end{array}$ & $\begin{array}{l}\text { L Tcb : Svb Tcb : } \\
\text { Nrb Tcb }\end{array}$ & 21.19 & $\begin{array}{l}\text { Location, Surgeon volume binned, Number of risks } \\
\text { binned }\end{array}$ \\
\hline $\begin{array}{l}\text { Knee, All } \\
\text { IVs }\end{array}$ & $\begin{array}{l}\text { L SNF : Fc SNF : } \\
\text { Ageb SNF : } \\
\text { Svb SNF : Nrb SNF : } \\
\text { Rmd SNF: Rug SNF: } \\
\text { Rhf SNF }\end{array}$ & 19.94 & $\begin{array}{l}\text { Location, financial class, age binned, surgeon volume } \\
\text { binned, number of risks binned, persistent mental } \\
\text { disorders (294.8), Unspecified glaucoma (365.9), } \\
\text { Heart Failure (428) }\end{array}$ \\
\hline $\begin{array}{l}\text { Hip, Como } \\
\text { IVs }\end{array}$ & $\begin{array}{l}\text { Rdi Cp : Ruh Cp : } \\
\text { Rhh Cp : Ram Cp : } \\
\text { Rra Cp : Rhe Cp : } \\
\text { Rrd Cp : Rca Cp : } \\
\text { Rhd Cp : Rpl Cp : } \\
\text { Rgp Cp : Rfr Cp }\end{array}$ & 15.35 & $\begin{array}{l}\text { Diabetes mellitus (250), Other and unspecified } \\
\text { hyperlipidemia (272.4), Hyposmolality and/or } \\
\text { hyponatremia (276.1), Unspecified deficiency anemia } \\
\text { (281.9), Alcohol abuse, in remission (305.03), } \\
\text { Unspecified essential hypertension (401.9), } \\
\text { Unspecified hypertensive renal disease (403.9), } \\
\text { Coronary atherosclerosis of native coronary artery } \\
\text { (414.01), Other chronic pulmonary heart diseases } \\
\text { (416.8), Hyperplasia of prostate (600), Repair of } \\
\text { cystocele with graft or prosthesis (70.54), Nonunion of } \\
\text { fracture (733.82) }\end{array}$ \\
\hline $\begin{array}{l}\text { Hip, All } \\
\text { IVs }\end{array}$ & $\begin{array}{l}\text { Ageb Cp : Nrb Cp : } \\
\text { Rrd Cp : Rca Cp : } \\
\text { Rhd Cp : Rpl Cp }\end{array}$ & 13.46 & $\begin{array}{l}\text { Age (binned), Number of risks (binned), Unspecified } \\
\text { hypertensive renal disease ( } 403.9) \text {, Coronary } \\
\text { atherosclerosis of native coronary artery ( } 414.01) \text {, } \\
\text { Other chronic pulmonary heart diseases (416.8), } \\
\text { Hyperplasia of prostate }(600)\end{array}$ \\
\hline $\begin{array}{l}\text { Knee, All } \\
\text { IVs }\end{array}$ & $\begin{array}{l}\text { Ageb Cp : Nrb Cp : } \\
\text { Ruh Cp:Rhd Cp : } \\
\text { Rku Cp : Rro Cp }\end{array}$ & 10.4 & $\begin{array}{l}\text { Age (binned), Number of risks (binned), Other and } \\
\text { unspecified hyperlipidemia (272.4), Other chronic } \\
\text { pulmonary heart disease (416.8), Chronic kidney } \\
\text { disease, unspecified (585.9), Rosacea (695.3) }\end{array}$ \\
\hline
\end{tabular}




\begin{tabular}{|c|c|c|c|}
\hline $\begin{array}{l}\text { Knee, } \\
\text { Como IVs }\end{array}$ & $\begin{array}{l}\text { Ruh Raf Cp : } \\
\text { Ros Cp : Rhd Cp : } \\
\text { Rav Cp : Reg Cp : } \\
\text { Rku Cp : Ruu Cp : } \\
\text { Rro Cp }\end{array}$ & 10.39 & $\begin{array}{l}\text { Other and unspecified hyperlipidemia (272.4) + Atrial } \\
\text { fibrillation (427.31), Obstructive sleep apnea (327.23), } \\
\text { Other chronic pulmonary heart disease (416.8), Aortic } \\
\text { valve disorders (424.1), Esophagitis (530.1), Chronic } \\
\text { kidney disease, unspecified (585.9), Other disorders of } \\
\text { urethra and urinary tract (599), Rosacea (695.3) }\end{array}$ \\
\hline $\begin{array}{l}\text { Hip, Como } \\
\text { IVs }\end{array}$ & $\begin{array}{l}\text { Rhy SNF : Rhh SNF : } \\
\text { Rug SNF :Rhe SNF : } \\
\text { Rrd SNF : } \\
\text { Rav Rbn SNF : } \\
\text { Rao SNF : Rse SNF : } \\
\text { Rnr SNF }\end{array}$ & 7.65 & $\begin{array}{l}\text { Unspecified acquired hypothyroidism (244.9), } \\
\text { Hyposmolality and/or hyponatremia (276.1), } \\
\text { Unspecified glaucoma (365.9), Unspecified essential } \\
\text { hypertension (401.9), Unspecified hypertensive renal } \\
\text { disease (403.9), Aortic valve disorders (424.1) + Other } \\
\text { disorders of bone and cartilage (733), Chronic airway } \\
\text { obstruction (496), Senile osteoporosis (733.01), Aseptic } \\
\text { necrosis of head and neck of femur (733.42) }\end{array}$ \\
\hline $\begin{array}{l}\text { Hip, All } \\
\text { IVs }\end{array}$ & $\begin{array}{l}\text { Rhp Re : Rdd Re : } \\
\text { Rpa Re : Rep Re : } \\
\text { Rhe Re }: \text { Rys Re : } \\
\text { Rki Re (by AIC, } \\
\text { p=.04) }\end{array}$ & 5.53 & $\begin{array}{l}\text { Hypopotassemia (276.8), Dysthymic disorder (300.4), } \\
\text { Parkinson's disease (332), Epilepsy (345.9), Essential } \\
\text { hypertension (401.9), Cardiac dysrhythmias (427), } \\
\text { Chronic kidney disease, Stage II (585.2) }\end{array}$ \\
\hline $\begin{array}{l}\text { Knee, } \\
\text { Como IVs }\end{array}$ & $\begin{array}{l}\text { Rhy SNF : Rau SNF : } \\
\text { Rmd SNF : Rpa } \\
\text { SNF : Rug SNF : } \\
\text { Rhe SNF : Rhf SNF : } \\
\text { Rku : Rbn SNF : } \\
\text { Rin SNF }\end{array}$ & 5.52 & $\begin{array}{l}\text { Hypothyroidism (244.9), Anemia (285.9), persistent } \\
\text { mental disorders (294.8), Parkinson's disease (332), } \\
\text { Unspecified glaucoma (365.9), Essential hypertension } \\
\text { (401.9), Heart failure (428), Chronic kidney disease } \\
\text { (585.9), Other disorders of bone and cartilage (733), } \\
\text { Urinary incontinence (788.3) }\end{array}$ \\
\hline $\begin{array}{l}\text { Hip, Como } \\
\text { IVs }\end{array}$ & $\begin{array}{l}\text { Rdd Re : Rpa Re : } \\
\text { Rep Re : Rhe Re : } \\
\text { Rys Re : Rer Re : } \\
\text { Rki Re (by AIC, } \\
\text { p=.03) }\end{array}$ & 5.52 & $\begin{array}{l}\text { Dysthymic disorder (300.4), Parkinson's disease (332), } \\
\text { Epilepsy (345.9), Essential hypertension (401.9), } \\
\text { Cardiac dysrhythmias (427), Esophageal reflux } \\
\text { (530.81), Chronic kidney disease, Stage II (585.2) }\end{array}$ \\
\hline $\begin{array}{l}\text { Knee, All } \\
\text { IVs }\end{array}$ & $\begin{array}{l}\text { Rci Re : Rco Re : } \\
\text { Rgp Re : Rcj Re }\end{array}$ & 3.77 & $\begin{array}{l}\text { Other forms of chronic ischemic heart disease (414), } \\
\text { Chronic obstructive asthma (493.2), Repair of cystocele } \\
\text { with graft or prosthesis (70.54), Contracture of joint, } \\
\text { lower leg ( } 718.46)\end{array}$ \\
\hline $\begin{array}{l}\text { Knee, } \\
\text { Como IVs }\end{array}$ & $\begin{array}{l}\text { Rci Re : Rco Re : } \\
\text { Rgp Re : Rcj Re }\end{array}$ & 3.77 & $\begin{array}{l}\text { Other forms of chronic ischemic heart disease (414), } \\
\text { Chronic obstructive asthma (493.2), Repair of cystocele } \\
\text { with graft or prosthesis (70.54), Contracture of joint, } \\
\text { lower leg (718.46) }\end{array}$ \\
\hline $\begin{array}{l}\text { Knee, } \\
\text { Como IVs }\end{array}$ & $\begin{array}{l}\text { Ruh Tcb : Rmo Tcb : } \\
\text { Rde Tcb : Ros Tcb : } \\
\text { Rhe Tcb }\end{array}$ & 1.51 & $\begin{array}{l}\text { Hyperlipidemia (272.4), Morbid obesity (278.01), } \\
\text { Depressive disorder (311), Obstructive sleep apnea } \\
\text { (327.23), Essential hypertension (401.9) }\end{array}$ \\
\hline $\begin{array}{l}\text { Hip, Como } \\
\text { IVs }\end{array}$ & $\begin{array}{l}\text { Rmo Tcb : Rcp Tcb : } \\
\text { Rrd Tcb : Rnr Tcb }\end{array}$ & 1.51 & $\begin{array}{l}\text { Morbid obesity (278.01), Chronic pain (338.29), } \\
\text { Hypertensive renal disease (403.9), Aseptic necrosis of } \\
\text { head and neck of femur (733.42) }\end{array}$ \\
\hline
\end{tabular}

The models listed above in Table 92 are the models selected by BIC, and do not readily demonstrate the importance of the individual predictors. In the next discussion, these predictors are discussed across the data sets and DVs. 


\section{Important Predictors of Outcome Variables}

One of the purposes of this research project was to determine the variables that were the most predictive of each of the DVs. A sample of previously known IVs was included in the data sets for this project, and results validated many as indeed important predictors, while excluding others. This finding serves to confirm the importance of previously known comorbidity variables. Additionally, the exploratory modeling approach used in this project sought to detect novel or surprising IVs that may not have been hypothesized previously in the literature. Indeed, a number of novel IVs were found to be important, and these are summarized in the following discussion.

In the search results for each of the DVs, the best models by both BIC and AIC were reported (the "search" action of Occam). While BIC was the criterion used for selecting the best models to analyze in detail (the "fit" action of Occam), the variables included in the AIC models have importance as well. Variables from both the BIC and AIC models were included in the three-tiered classification of results after the search results in each of the DV sections. In this three-tiered classification, the IVs from both All IVs and the Comorbidity IVs are integrated. The Tier 1 variables include the most important predicting variables, which are those that were included in the best model by $\mathrm{BIC}$ in the All IVs data set. The Tier 2 variables are those additional variables that appeared both in the best model by AIC in the combined All IVs search, and in the best model by BIC in the Comorbidity IVs search. Tier 3 includes those variables that occurred in either of the two searches from Tier 2 but not both.

To get a better understanding of the importance of variables throughout all of the analyses, a simple ranking system was created as follows. Every time an IV was flagged 
as a Tier 1 variable, it was assigned 3 points, Tier 2 IVs were assigned 2 points, and Tier 3 IVs were assigned 1 point. When applied across the 4 DVs and across both the hip and knee data sets, this simple point system shows which IVs were the most influential overall (Table 93). The single most influential IV is number of risks binned (Nrb), which shows up as a Tier 1 IV in both hip and knee searches for Complication (Cp), Skilled Nursing Facility (SNF), and Total Cost (Tcb). The second most influential variable is patient age (Ageb), which shows up as a Tier 1 variable in both the hip and knee searches for Cp and SNF. The top five most influential IVs were from All IVs, which were seen to have significant effects in the prediction of the DVs. Of the IVs with an assigned point value of 5 or greater, only hypertensive renal disease (Rrd) was not shared across the hip and knee searches; however, Rrd is an important variable for hip cases for the DVs Cp (Complication), SNF (Skilled Nursing Facility), and Tcb (Total Cost Binned). 
Table 93. Top Important IVs across Hip and Knee Data Sets, and across the 4 DVs.

$(\mathrm{Cp}=$ Complication, $\mathrm{SNF}=$ Skilled Nursing Facility, $\mathrm{Re}=$ Readmit, and Tcb $=$ Total Cost Binned.$)$

Novel variables are indicated by *.

The Shared column indicates IVs located in both hip and knee data sets.

\begin{tabular}{|c|c|c|c|c|c|c|c|c|c|c|c|}
\hline & & 정 & 疍 & 중 & 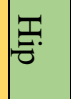 & 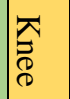 & $\underline{\Xi}$ & 중 & $\Xi$ & & \\
\hline IV ID & Variable Name & $\approx$ & 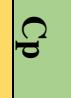 & $\sum_{x}^{2}$ & $\frac{2}{x}$ & $\ddot{0}$ & $\ddot{0}$ & $\frac{2}{2}$ & $\vec{a}$ & Shared & $\begin{array}{l}\text { Total } \\
\text { Points }\end{array}$ \\
\hline $\mathrm{Nrb}$ & Number of Risks (binned) & 1 & 1 & 1 & 1 & & & 1 & 1 & yes & 18 \\
\hline Ageb & Age (binned) & 1 & 1 & 1 & 1 & & & & & yes & 12 \\
\hline $\mathrm{Svb}$ & Surgeon volume (binned) & & & 1 & 1 & & & & 1 & yes & 9 \\
\hline $\mathrm{L}$ & Location & & & 1 & & & & & 1 & yes & 6 \\
\hline Fc & Financial class & & & 1 & 1 & & & & & yes & 6 \\
\hline Rhd & Other chronic pulmonary heart diseases & 1 & 1 & & & & & & & yes & 6 \\
\hline Rhe & Unspecified essential hypertension & & 3 & 3 & 3 & & 3 & 3 & & yes & 5 \\
\hline Ruh & Other and unspecified hyperlipidemia & 1 & 3 & & & & & 3 & & yes & 5 \\
\hline Rrd & Unspecified hypertensive renal disease & & 1 & & 3 & & & & 3 & & 5 \\
\hline Rgp & Repair of cystocele with graft or prosthesis & & 2 & & & 1 & & & & yes & 5 \\
\hline *Rug & Unspecified glaucoma & & & 1 & 3 & & & 3 & & yes & 5 \\
\hline$S$ & Surgeon & & & & & & & 1 & 3 & yes & 4 \\
\hline Rca & $\begin{array}{l}\text { Coronary atherosclerosis of native coronary } \\
\text { artery }\end{array}$ & & 1 & & & 3 & & & & yes & 4 \\
\hline Rku & Chronic kidney disease, unspecified & 1 & & 3 & & & & & & & 4 \\
\hline Rci & Chronic ischemic heart disease & & & & & 1 & & & & & 3 \\
\hline Rhf & Heart failure & & & 1 & & & & & & & 3 \\
\hline Rav & Aortic valve disorders & 2 & & & 3 & & & & & yes & 3 \\
\hline Rmo & Morbid obesity & & & & & & & 2 & 3 & yes & 3 \\
\hline Rcj & Contracture of joint, lower leg & & & & & 1 & & & & & 3 \\
\hline Rco & Chronic obstructive asthma & & & & & 1 & & & & & 3 \\
\hline$*$ Rmd & $\begin{array}{l}\text { Other persistent mental disorders due to } \\
\text { conditions classified elsewhere }\end{array}$ & & & 1 & & & & & & & 3 \\
\hline *Rpa & Parkinson's disease & & & 2 & & & 3 & & & yes & 3 \\
\hline *Rhy & Unspecified acquired hypothyroidism & & & 3 & 2 & & & & & yes & 3 \\
\hline$* \mathrm{Rpl}$ & Hyperplasia of prostate & & 1 & & & & & & & & 3 \\
\hline *Ros & Obstructive sleep apnea & 2 & & & & & & 3 & & & 3 \\
\hline Raf & Atrial fibrillation & 2 & & & & & & & & & 2 \\
\hline Rur & Retention of urine & & & & 2 & & & & & & 2 \\
\hline Ruu & Other disorders of urethra and urinary tract & 2 & & & & & & & & & 2 \\
\hline$*$ Rnn & Nephritis and nephropathy & & & & 3 & & & 3 & & yes & 2 \\
\hline *Rep & Epilepsy unspecified & & & & & 3 & 3 & & & yes & 2 \\
\hline *Reg & Esophagitis & 2 & & & & & & & & & 2 \\
\hline$* \mathrm{Rhh}$ & Hyposmolality and/or hyponatremia & & 3 & & 3 & & & & & & 2 \\
\hline Rdf & Chronic diastolic heart failure & & & & & 3 & & & & & 1 \\
\hline Rht & Heart disease, unspecified & & & & & & & 3 & & & 1 \\
\hline Rys & Cardiac dysrhythmias & & & & & & 3 & & & & 1 \\
\hline Rdi & Diabetes mellitus & & 3 & & & & & & & & 1 \\
\hline Rki & Chronic kidney disease, Stage II (mild) & & & & & & 3 & & & & 1 \\
\hline Rin & Urinary incontinence & & & 3 & & & & & & & 1 \\
\hline Rbn & Other disorders of bone and cartilage & & & 3 & & & & & & & 1 \\
\hline Rnr & Aseptic necrosis of head and neck of femur & & & & & & & & 3 & & 1 \\
\hline
\end{tabular}




\begin{tabular}{|c|c|c|c|c|c|c|c|c|c|c|c|}
\hline & & $\begin{array}{l}\text { 중 } \\
\text { 尺े } \\
\bar{\varnothing}\end{array}$ & Eִ & 중 & 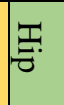 & 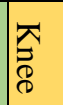 & $\underline{E}$ & $\begin{array}{l}\text { 중 } \\
\text { ఫ్ } \\
\end{array}$ & $\Xi$ & & \\
\hline IV ID & Variable Name & $f$ & $\approx$ & 至 & 告 & $\approx$ & 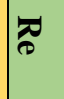 & $\overrightarrow{2}$ & $\overrightarrow{2}$ & Shared & $\begin{array}{l}\text { Total } \\
\text { Points }\end{array}$ \\
\hline Rfr & Nonunion of fracture & & 3 & & & & & & & & 1 \\
\hline $\mathrm{Rmn}$ & Mononeuritis of unspecified site & & & & & & & 3 & & & 1 \\
\hline Rse & Senile osteoporosis & & & & 3 & & & & & & 1 \\
\hline Rao & Chronic airway obstruction & & & & 3 & & & & & & 1 \\
\hline Rep & Other chronic pain & & & & & & & & 3 & & 1 \\
\hline *Rde & Depressive disorder & & & & & & & 3 & & & 1 \\
\hline$* \mathrm{Rbp}$ & Other and unspecified bipolar disorders & & & 3 & & & & & & & 1 \\
\hline *Rdd & Dysthymic disorder & & & & & & 3 & & & & 1 \\
\hline *Rra & Alcohol abuse, in remission & & 3 & & & & & & & & 1 \\
\hline$* \mathrm{Rml}$ & Memory loss & & & & 3 & & & & & & 1 \\
\hline$*$ Rhp & Hypopotassemia & & & & & & 3 & & & & 1 \\
\hline$* \mathrm{Rau}$ & Anemia, unspecified & & & 3 & & & & & & & 1 \\
\hline *Ram & Unspecified deficiency anemia & & 3 & & & & & & & & 1 \\
\hline *Rro & Rosacea & 3 & & & & & & & & & 1 \\
\hline IV ID & Variable Name & fo & o & 章 & $\frac{z}{\mathbf{Z}}$ & $\approx$ & $\pi$ & $\frac{\overrightarrow{2}}{2}$ & $\frac{1}{2}$ & Shared & $\begin{array}{l}\text { Total } \\
\text { Points }\end{array}$ \\
\hline
\end{tabular}

Recall that some of the IVs used in the final data sets for the exploratory searches were retained because they had an individual predictive value with $\mathrm{p}$-value $\leq 0.05$. Additionally, recall that there were some IVs that were kept, not because of their predictive value in the analysis of this project, but because they were found to be predictive in the literature (Table 9). There is an extensive literature that discusses additional comorbidities for joint replacement and other surgeries that does not offer actual predictive models that include them. These were not flagged as "literature-based IVs" in this project, yet they are also not "surprising." For example, in Table 93, the IV hypertensive renal disease ( $\mathrm{Rrd}$ ) is important for $\mathbf{C p}, \mathbf{S N F}$, and $\mathbf{T c b}$. The IV Rrd was not used in any of the predictive models from the literature; however, diabetes was. Clinically, it is well known that diabetes is associated with, and may lead to, renal 
disease, and therefore it is not that surprising or novel that hypertensive renal disease (Rrd) shows up as having an effect in the exploratory searches in this project.

However, there are 19 predictive IVs in Table 93 that seem to be surprising and novel. An asterisk next to an IV name in Table 93 indicates that the IV was a novel variable that has not been present in the literature of hip and knee outcomes thus far. For example, unspecified glaucoma (Rug) is not a comorbidity found in the literature (Table 9) to be predictive of outcomes in hip or knee replacement, or for other surgeries. These novel IVs should be considered in future confirmatory research, and their clinical relevance should be explored.

\section{Patient Variables versus Delivery System Variables}

Predictive analytics in healthcare is still relatively new, but the use of predictive tools is going to play an increasingly important role in measuring quality and the provisioning of resources in care delivery. In healthcare analytics today, predictive analytics are often discussed as methods to predict outcomes per patient, given a set of patient-specific clinical diagnoses (and more recently genomic data). Without a doubt, personalized medicine will increase providers' ability to diagnose with better accuracy and apply more appropriate targeted therapies.

The delivery system, however, has hardly turned the lens on itself. Applying advanced predictive modeling techniques on patient level data, without including data about the way care is delivered, will miss large opportunities. This research showed that by looking at variables related to the delivery system (hospital, surgeon, surgeon volume), there is quite a bit of information that clearly shows, regardless of the patient's 
risk profile, that the delivery system itself is highly predictive of discharge practices and costly outcomes.

In this section of the discussion, variables are now partitioned into either PatientRelated IVs or Delivery System IVs. This partitioning of IVs is not the same as the All IVs and Comorbidity IVs schema. The rationale for this is to see how much of the predicted "risk." per outcome, is really attributable to a patient's risk versus the amount of "risk" that is actually attributable to the Delivery System itself-in other words, the manner in which we deliver care.

The Comorbidity IVs were the binary, individual patient comorbidities that were generated from the ICD-9 diagnosis codes that were indicated as present on admit. All IVs contained both the individual patient Comorbidity IVs in addition to noncomorbidity patient-related IVs and delivery system-centered IVs. The IVs from All IVs that are non-comorbidity patient-related are number of risks binned (Nrb), patient age (Ageb), and admit diagnosis (Ad). These are now considered alongside the individual Comorbidity IVs as Patient-Related (Figure 17). The All IVs that are delivery systemcentered are location (L), surgeon (S), surgeon volume (Svb), and day of admit (Da) and are now assigned as Delivery System.

\begin{tabular}{|c|c|c|}
\hline \multicolumn{3}{|c|}{ All IVs } \\
\hline & & Comorbidity IVs \\
\hline Delivery System-Centered & Non-comorbidity, Patient-Related & Comorbidities \\
\hline Delivery System IVs & \multicolumn{2}{|c|}{ Patient-Related IVs } \\
\hline
\end{tabular}

Figure 17. New Partition of IVs into Delivery System and Patient-Related IVs. 
In the introduction to this project, there was a discussion of the emerging trend of providers (for knee and hip replacements, the surgeons) to be increasingly contracted under value-based payment models that reimburse for delivering high-quality outcomes, often without taking into consideration the differential risks posed by varying comorbidities (patient risk) in the patient population. One of the perceived and unintended side effects of this is what is called "cherry picking," where some providers select patients with no or few comorbidities and therefore have healthier patient populations with a lower risk of adverse outcomes. Similarly, refraining from cherry picking behavior would result in a patient population that would have a greater risk of adverse outcomes than their cherry-picked peers. Often, there is a suspicion that cherry picking may be occurring, but little evidence is provided to support this argument. Regardless, one cannot make a fair or valid comparison of outcomes across providers without looking at patient risk.

The results of this project show that the Delivery System itself plays a role in these outcomes. The surgeon, the surgeon's volume, and the location were all very predictive of SNF and Tcb. If patients seeking care are being turned away because of their comorbidities out of concern that they will result in higher-cost care, then the results that the delivery system is more predictive of the total cost than patient comorbidities has implications.

The best model predicting Complication (Cp) includes IVs that are Comorbidity IVs as well as non-comorbidity IVs within All IVs. From the best model Ageb Cp : $\operatorname{Nrb} \mathbf{C p}$ : Ruh $\mathbf{C p}$ : Rhd $\mathbf{C p}$ : Rku $\mathbf{C p}$ : Rro $\mathbf{C p}$, two submodels were generated; one with the subset of IVs that are Delivery System only, and one with IVs that are Patient- 
Related only. Separate Occam runs were then conducted, and the $\% \Delta \mathrm{H}$ of each of the new submodels is presented in Table 94 below. Looking at the DV Complication (Cp) for the knee patients, Table 94 shows that all of the information in the model $(\% \Delta \mathrm{H})$ is attributed to the Patient-Related IVs. In other words, it is indeed the patient's individual comorbidities that predict Complication $(\mathbf{C p})$. This is also true for the hip replacement patients.

Table 94. Delivery System vs. Patient-Related Submodels Comparison of Reduction of Uncertainty for DV Complication (Cp).

\begin{tabular}{|l|l|l|}
\hline DV: Complication (Cp) \\
\hline \multirow{5}{*}{ Knee } & Full Model by BIC for Cp: & $\% \Delta \mathrm{H}$ (Total) \\
\cline { 2 - 3 } & IV:AgebCp:NrbCp:CpRuh:CpRhd:CpRku:CpRro & 10.40 \\
\cline { 2 - 3 } & Patient Related IVs: & $\% \Delta \mathrm{H}$ (patient-related) \\
\cline { 2 - 3 } & IV:AgebCp:NrbCp:CpRuh:CpRhd:CpRku:CpRro & 10.40 \\
\cline { 2 - 3 } & Delivery System IVs: & $\% \Delta \mathrm{H}$ (delivery system) \\
\cline { 2 - 3 } & None & 0.00 \\
\hline \multirow{5}{*}{ Hip } & Best Model by BIC for Cp: & $\% \Delta \mathrm{H}$ (Total) \\
\cline { 2 - 3 } & IV:AgebCp:NrbCp:CpRrd:CpRca:CpRhd:CpRpl & 13.46 \\
\cline { 2 - 3 } & Patient Related IVs: & $\% \Delta \mathrm{H}$ (patient-related) \\
\cline { 2 - 3 } & IV:AgebCp:NrbCp:CpRrd:CpRca:CpRhd:CpRpl & 13.46 \\
\cline { 2 - 3 } & Delivery System IVs: & 0.00 \\
\cline { 2 - 3 } & None & \\
\hline
\end{tabular}

When looking at the DV Skilled Nursing Facility (SNF), there is a bit of a mix of Patient-Related and Delivery System IVs, although the majority of the information is provided by the Patient-Related IVs (Table 95). Note that the reductions of uncertainty are not expected to be strictly additive. For example, in the Knee values of Table 95, 19.94 is not the sum of $16.08+3.14$. Entropy and entropy reduction numbers are not expected to show linearity or additivity; they are nonlinear expressions. 
Table 95. Delivery System vs. Patient-Related Submodels Comparison of Reduction of Uncertainty for DV Skilled Nursing Facility (SNF).

\begin{tabular}{|l|l|l|}
\hline DV: Skilled Nursing Facility (SNF) & $\% \Delta \mathrm{H}$ (Total) \\
\hline \multirow{5}{*}{ Knee } & Full Model by BIC for SNF: & 19.94 \\
\cline { 2 - 3 } & IV:LSNF:FcSNF:SNFAgeb:SNFSvb: & \\
\cline { 2 - 3 } SNFNrb:SNFRmd:SNFRug:SNFRhf & Patient Related IVs: & $\% \Delta \mathrm{H}$ (patient-related) \\
\cline { 2 - 3 } & IV:FcSNF:SNFAgeb:SNFNrb:SNFRmd:SNFRug:SNFRhf & 16.08 \\
\cline { 2 - 3 } & Delivery System IVs: & $\% \Delta \mathrm{H}$ (delivery system) \\
\cline { 2 - 3 } & IV:LSNF:SNFSvb & 3.14 \\
\hline \multirow{5}{*}{ Hip } & Best Model by BIC for SNF: & $\% \Delta \mathrm{H}$ (Total) \\
\cline { 2 - 3 } & IV:FcSNF:SNFAgeb:SNFSvb:SNFNrb & 23.61 \\
\cline { 2 - 3 } & Patient Related IVs: & $\% \Delta \mathrm{H}$ (patient-related) \\
\cline { 2 - 3 } & IV:FcSNF:SNFAgeb:SNFNrb & 17.21 \\
\cline { 2 - 3 } & Delivery System IVs: & 7.53 \\
\cline { 2 - 3 } & IV:SNFSvb & $\mathrm{H}$ (delivery system) \\
\hline
\end{tabular}

Readmission is not very well predicted, but when it is, the information is captured by Patient-Related IVs (Table 96), as there are no IVs in the model that are either

Delivery System IVs or even All IVs.

Table 96. Delivery System vs. Patient-Related Submodels Comparison of Reduction of Uncertainty for DV Readmission (Re).

\begin{tabular}{|l|l|l|}
\hline DV: Readmission (Re) & $\% \Delta \mathrm{H}$ (Total) \\
\hline \multirow{4}{*}{ Knee } & Full Model by BIC for Re: & 3.77 \\
\cline { 2 - 3 } & IV:RciRe:RcoRe:RgpRe:RcjRe & $\% \Delta \mathrm{H}$ (patient-related) \\
\cline { 2 - 3 } & Patient Related IVs: & 3.77 \\
\cline { 2 - 3 } & IV:RciRe:RcoRe:RgpRe:RcjRe & $\% \Delta \mathrm{H}$ (delivery system) \\
\cline { 2 - 3 } & Delivery System IVs: & 0.00 \\
\cline { 2 - 3 } & None & $\% \Delta \mathrm{H}$ (Total) \\
\hline \multirow{5}{*}{ Hip } & Best Model by BIC for Re: & 5.53 \\
\cline { 2 - 3 } & IV:RhpRe:RddRe:RpaRe:RepRe:RheRe:RysRe:RkiRe & $\% \Delta \mathrm{H}$ (patient-related) \\
\cline { 2 - 3 } & Patient Related IVs: & 5.53 \\
\cline { 2 - 3 } & IV:RhpRe:RddRe:RpaRe:RepRe:RheRe:RysRe:RkiRe & $\% \Delta \mathrm{H}$ (delivery system) \\
\cline { 2 - 3 } & Delivery System IVs: & 0.00 \\
\cline { 2 - 3 } & None & \\
\hline
\end{tabular}

The analysis of the DV total cost differs considerably from the other DVs in the relative importance of Patient-Related versus Delivery System IVs. Recall the concern that sicker patients cost more and the subsequent cherry picking behavior. By partitioning the IVs, it is shown that nearly all of the information about total cost $(\mathrm{Tcb})$ is attributed to 
the Delivery System (i.e., Surgeon) and not the patient's risk (i.e., Nrb). In fact, for the knee patients, the $\% \Delta \mathrm{H}$ using only the Delivery System IV surgeon (S) is 23.71 (Table 97) and the Patient-Related IV, number of risks (Nrb), only reduces uncertainty by $1.82 \%$. This predictive effect of surgeon $(\mathrm{S})$ could be due to either the surgeon being a more efficient provider or an artifact of the surgeon "cherry picking" and selecting healthier patients with lesser numbers of risks (Nrb). An Occam search was thus performed looking at the predictive effect of $\mathrm{Nrb}$ on $\mathrm{S}$, and the resulting $\% \Delta \mathrm{H}$ for knee data was 3.24 and 2.32 for hip — not a strong association. There does not appear to be strong evidence for cherry picking in this project's data set. Rather, the efficiency of surgeons (i.e their practice patterns) is a likely culprit. Measures of surgeon efficiency would be good additions for an enhanced delivery-system data set. For the Hip patients it is a similar scenario, where the Delivery System IVs location (L) and surgeon volume (Svb) reduce uncertainty by $19.71 \%$, while Patient-Related IVs number of risks (Nrb) reduce uncertainty by $0.88 \%$ (Table 97 ). Location (L) alone reduces uncertainty of Tcb by $13.44 \%$, and surgeon volume (Svb) by $13.15 \%$ as seen below in Table 97 . 
Table 97. Delivery System vs. Patient-Related Submodels Comparison of Reduction of Uncertainty for DV Total Cost (Tcb).

\begin{tabular}{|c|c|c|}
\hline \multicolumn{3}{|c|}{ DV: Total Cost (Tcb) } \\
\hline \multirow{6}{*}{ Knee } & Full Model by BIC for Tcb: & $\% \Delta \mathrm{H}($ Total $)$ \\
\hline & IV:STcb:TcbNrb & 25.13 \\
\hline & Patient Related IVs: & $\% \Delta H$ (patient-related) \\
\hline & IV:TcbNrb & 1.82 \\
\hline & Delivery System IVs: & $\% \Delta \mathrm{H}$ (delivery system) \\
\hline & IV:STcb & 23.71 \\
\hline \multirow{4}{*}{ Hip } & Best Model by BIC for Tcb: & $\% \Delta \mathrm{H}($ Total $)$ \\
\hline & IV:LTcb:TcbSvb:TcbNrb & 21.19 \\
\hline & Patient Related IVs: & $\% \Delta \mathrm{H}$ (patient-related) \\
\hline & IV:TcbNrb & 0.88 \\
\hline & Delivery System IVs: & $\% \Delta \mathrm{H}$ (delivery system) \\
\hline & IV:LTcb:TcbSvb & 19.71 \\
\hline & $>$ IV:LTcb & 13.44 \\
\hline & $>\mathrm{IV}: \mathrm{SvbTcb}$ & 13.15 \\
\hline
\end{tabular}

The effect of delivery system variables on total cost is sizeable. Larger and larger data repositories are being cultivated by health systems, and interoperability between electronic health record systems, in addition to the collection of patient-reported health data and increasing genomic data collection, will result in increasingly rich data sets. However, as tentatively suggested from the results of the analysis in this project, increasing the quantity of patient-related data may not provide better predictions. Total Cost in particular was predicted by variables in the domain of the delivery system. In this project, a very small subset of possible delivery system IVs were used. As practitioners move forward with developing predictive analytics in healthcare, new domains of data measuring how the healthcare system delivers care ought to be created and included in these efforts. These variables might represent surgeon efficiency (e.g., implant selection, dedicated OR staff, surgical time, etc.) or care delivery processes (time to ambulation, pain management techniques, preoperative patient education, etc.). Future research 
should be conducted to further identify additional variables representative of the delivery system and tested for their effect on outcomes.

Readmissions were not well predicted by this project's data. Possibilities are either (a) that the delivery system and patient comorbidities are not the correct domains informative of readmissions, or (b) readmissions are unpredictable. In order to test the former, additional data should be generated. There is some evidence that patient social and demographic factors (e.g., living alone, income, education, etc.) and historic utilization patterns (e.g., number of ED visits in prior 12 months) are helpful in predicting readmissions; however, this data is not readily available by health systems.

\section{Classifying Patients as Higher or Lower Risk for Adverse Outcomes}

Predictive models can augment clinical decision-making by providing additional information. The models resulting from this research provide new information about risk for a sizeable proportion of the patient population. Table 98 summarizes, for all the DVs, the percent of patients in the sample who are at increased and decreased risk, where this increase or decrease of risk is defined as more than a $10 \%$ difference between the conditional probability of an adverse outcome given the predictors and the marginal probability of this outcome, where this difference is statistically significant at the 0.05 level. 
Table 98. Summary of Decreased and Increased IV States by DV

(Cp=Complication, SNF=Skilled Nursing Facility, Re=Readmission, Tcb=Total Cost).

\begin{tabular}{|c|c|c|c|c|c|}
\hline \multicolumn{6}{|c|}{ Knee } \\
\hline & & $\mathrm{Cp}$ & SNF & $\operatorname{Re}$ & Tcb \\
\hline \multirow{2}{*}{ All IVs } & Decreased Risk IV States & $40.50 \%$ & $25.90 \%$ & $0.00 \%$ & $21.86 \%$ \\
\hline & Increased Risk IV States & $15.73 \%$ & $19.63 \%$ & $4.64 \%$ & $21.49 \%$ \\
\hline & $\%$ Total & $56.23 \%$ & $45.53 \%$ & $4.64 \%$ & $43.35 \%$ \\
\hline \multirow{2}{*}{ Comorbidity IVs } & Decreased Risk IV States & $76.36 \%$ & $75.46 \%$ & $0.00 \%$ & $0.00 \%$ \\
\hline & Increased Risk IV States & $16.86 \%$ & $13.93 \%$ & $4.64 \%$ & $0.00 \%$ \\
\hline & $\%$ Total & $93.22 \%$ & $89.39 \%$ & $4.64 \%$ & $0.00 \%$ \\
\hline \multicolumn{6}{|c|}{ Hip } \\
\hline & & $\mathrm{Cp}$ & SNF & $\operatorname{Re}$ & Tcb \\
\hline \multirow{2}{*}{ All IVs } & Decreased Risk IV States & $47.27 \%$ & $46.41 \%$ & $52.50 \%$ & $28.30 \%$ \\
\hline & Increased Risk IV States & $13.51 \%$ & $24.69 \%$ & $1.22 \%$ & $21.53 \%$ \\
\hline & $\%$ Total & $60.78 \%$ & $71.10 \%$ & $53.72 \%$ & $49.83 \%$ \\
\hline \multirow{2}{*}{ Comorbidity IVs } & Decreased Risk IV States & $71.98 \%$ & $42.79 \%$ & $45.69 \%$ & $0.00 \%$ \\
\hline & Increased Risk IV States & $3.43 \%$ & $11.45 \%$ & $0.56 \%$ & $0.34 \%$ \\
\hline & $\%$ Total & $75.41 \%$ & $54.24 \%$ & $46.25 \%$ & $0.34 \%$ \\
\hline
\end{tabular}

In the summary of results that follows, all of the patients at increased or decreased risk, relative to the margins, across the different IV states for each model are tallied up, and the percent of the whole sample is calculated. Additionally, a weighted average of the risk ratios (weighted by the frequencies of the IV states) shows the average risk across the multiple IV states in the model. While the fraction of patients at increased or decreased risk is statistically significant, the average risk ratio has not been subjected to any statistical test. All models discussed in this section are from the All IVs searches, as individual patient comorbidities are included within All IVs.

\section{Complications}

For example, a complication (Cp) was observed in $4.73 \%$ (32 patients) of the 4,336 patients in the knee data set. The simplest model based on this value, taking into account nothing else about the patients or delivery system, would predict that $4.73 \%$ of knee patients will experience complications. However, the best model from this project's 
analyses (Ageb Cp : Nrb Cp : Ruh $\mathbf{C p}$ : Rhd $\mathbf{C p}$ : Rku Cp : Rro Cp) identified several groups of patients who were at increased risk of $\mathbf{C p}$ with particular combinations of IV states from the model (Table 24). Considering these high-risk groups together, $15.73 \%$ of the total patients in the sample had an increased risk of complication (Table 99). For these patients at increased risk, the weighted average risk ratio is 2.41 ; thus $11.40 \%$ (or 78 patients) out of that group (15.73\% of the whole) would be predicted to experience complications (that is, 2.41 times the observed marginal complication rate of $4.73 \%$ for the knee data set).

Table 99. Increased and Decreased Risk IV States for Complication (Cp).

\begin{tabular}{|c|c|c|c|c|}
\hline \multicolumn{3}{|l|}{ Complication (Cp) } & & \\
\hline \multicolumn{5}{|l|}{ Knee $($ All IVs) } \\
\hline $\begin{array}{l}\text { Model: Ageb Cp : Nrb Cp : Ruh Cp : Rhd Cp : } \\
\text { Rku Cp : Rro Cp }\end{array}$ & Freq & $\%$ of Cases & Ratio Average & Margin \\
\hline Increased Risk IV States & 682 & $15.73 \%$ & 2.41 & 11.40 \\
\hline Decreased Risk IV States & 1756 & $40.50 \%$ & 0.32 & 1.51 \\
\hline No difference (by significance or frequency) & 1898 & $43.77 \%$ & & \\
\hline Total & 3654 & & & 4.73 \\
\hline \multicolumn{5}{|l|}{ Hip (All IVs) } \\
\hline $\begin{array}{l}\text { Model: Ageb Cp : Nrb Cp : Rrd Cp : Rca Cp : Rhd Cp } \\
\text { Rpl Cp }\end{array}$ & Freq & $\%$ of Cases & Ratio Average & Margin \\
\hline Increased Risk IV States & 433 & $13.51 \%$ & 2.88 & 14.75 \\
\hline Decreased Risk IV States & 1515 & $47.27 \%$ & 0.34 & 1.74 \\
\hline No difference (by significance or frequency) & 1257 & $39.22 \%$ & & \\
\hline Total & 3205 & & & 5.12 \\
\hline
\end{tabular}

Not only do the risk groups show the IV states that put patients at greater risk of having a complication $(\mathbf{C p})$, but they show the groups of patients who were at decreased risk. There were several groups of knee patients (Table 24) whose IV states for the model Ageb Cp : Nrb Cp : Ruh Cp : Rhd Cp : Rku Cp : Rro Cp predicted a decreased risk of Cp (blue rows). Added together, $40.50 \%$ of the knee patients were identified as at decreased risk of $\mathbf{C p}$ (Table 99). Of these patients, $1.51 \%$ would be expected to have complications compared to the observed rate of $4.73 \%$ for the entire sample. 
For the patients with hip replacements, the best model predicting a complication, Ageb Cp : Nrb Cp : Rrd Cp : Rca Cp : Rhd Cp : Rpl Cp, uncovered IVs whose states resulted in $13.51 \%$ of the patients identified as at higher risk of $\mathbf{C p}$ (Table 99). Of these, $14.75 \%$ would be expected to have $\mathbf{C p}$, which was 2.88 times the observed rate of $5.12 \%$ for the entire hip data set. Of the hip patients, 47.27 would be expected to have decreased risk of $\mathbf{C p}$, with $1.74 \%$ expected to have $\mathbf{C p}$.

\section{Discharge to skilled nursing facility}

The best All IVs models predicting discharge to a skilled nursing facility (SNF) also presented IVs whose combination of states resulted in higher risk or lower risk scenarios for each specific combination of states. These IV states were analyzed in the models' fit, (detailed in Table 49 and Table 57). These tables presented rows where the IV states of the model resulted in increased risk (orange) and decreased risk (blue). Considered together, the IV states can be tallied up to show the percentage of patients in each of the risk groups, their actual rate of discharge to SNF, as well as the average risk ratio per risk group.

Table 100 summarizes the risk groups of each model for SNF across both the hip and the knee data sets. The best All IVs model in the knee data set for SNF resulted in $19.63 \%$ of the total sample expected to be at increased risk with an expected SNF rate of 39.66, much higher than the observed rate of $17.55 \%$ for the entire sample. Lower risk of SNF was predicted for $25.90 \%$ of knee cases, of which $3.34 \%$ would be expected to be discharged to a SNF (compared to the observed rate of $17.55 \%$ of the entire knee sample). 
Table 100. Increased and Decreased Risk IV States for Skilled Nursing Facility (SNF)

\begin{tabular}{|c|c|c|c|c|}
\hline SNF & & & & \\
\hline Knee (All IVs) & & & & \\
\hline $\begin{array}{l}\text { Model: L SNF : Fc SNF : Ageb SNF : Svb SNF : Nrb SNF } \\
\text { : Rmd SNF : Rug SNF : Rhf SNF }\end{array}$ & Freq & $\%$ of Cases & Ratio Average & Margin \\
\hline Increased Risk IV States & 851 & $19.63 \%$ & 2.26 & 39.66 \\
\hline Decreased Risk IV States & 1123 & $25.90 \%$ & 0.19 & 3.34 \\
\hline No difference (by significance or frequency) & 2362 & $54.47 \%$ & & \\
\hline Total & 4336 & & & 17.55 \\
\hline Hip (All IVs) & & & & \\
\hline Model: Fc SNF : Ageb SNF : Svb SNF : Nrb SNF & Freq & $\%$ of Cases & Ratio Average & Margin \\
\hline Increased Risk IV States & 791 & $24.69 \%$ & 2.71 & 38.65 \\
\hline Decreased Risk IV States & 1487 & $46.41 \%$ & 0.19 & 2.71 \\
\hline No difference (by significance or frequency) & 926 & $28.90 \%$ & & \\
\hline Total & 3204 & & & 14.26 \\
\hline
\end{tabular}

The All IVs model for the hip data set placed $24.69 \%$ in a high-risk group and $46.41 \%$ of patients in a lower-risk group (Table 100). Of these patients, the high-risk group had an expected SNF rate of $38.65 \%$ compared with the overall observed SNF rate for the entire hip sample of $14.26 \%$, while the low-risk group had an expected SNF rate of $2.71 \%$.

\section{Readmissions}

Readmissions were not as predictable as the DVs Cp and SNF. For the knee data, $4.64 \%$ of patients were in a higher-risk group compared to the observed readmission of $2.61 \%$ of the overall knee data set. Of the higher-risk patients identified, $9.11 \%$ had a predicted readmission ( $\mathbf{R e})$ on the basis of an average risk ratio of 3.49 (Table 101). There were no combinations of IV states that resulted in patients being considered at lower risk of $\mathbf{R e}$. 
Table 101. Increased and Decreased Risk IV States for Readmission (Re).

\begin{tabular}{|c|c|c|c|c|}
\hline \multicolumn{5}{|l|}{ Readmission } \\
\hline \multicolumn{5}{|l|}{ Knee (All IVs) } \\
\hline Model: Rci Re : Rco Re : Rgp Re : Rcj Re & Freq & $\%$ of Cases & Ratio Average & Margin \\
\hline Increased Risk IV States & 201 & $4.64 \%$ & 3.49 & 9.11 \\
\hline Decreased Risk IV States & 0 & $0.00 \%$ & & \\
\hline No difference (by significance or frequency) & 4133 & $95.36 \%$ & & \\
\hline 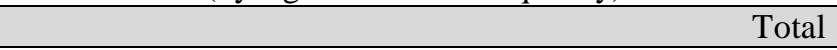 & 4334 & & & 2.61 \\
\hline \multicolumn{5}{|l|}{ Hip $(A l l ~ I V s)$} \\
\hline $\begin{array}{l}\text { Model: Rhp Re : Rdd Re : Rpa Re : Rep Re : Rhe Re : } \\
\text { Rys Re : Rki Re }\end{array}$ & Freq & $\%$ of Cases & Ratio Average & Margin \\
\hline Increased Risk IV States & 39 & $1.22 \%$ & 4.79 & 13.03 \\
\hline Decreased Risk IV States & 1680 & $52.50 \%$ & 0.64 & 1.74 \\
\hline No difference (by significance or frequency) & 1481 & $46.28 \%$ & & \\
\hline Total & 3200 & & & 2.72 \\
\hline
\end{tabular}

The average readmission ( $\mathbf{R e})$ rate for the overall hip sample was $2.72 \%$. The best models for the hip data set for readmission (Re) identified groups of patients both at higher risk and at lower risk of readmission with $1.22 \%$ of patients being classified into the higher-risk group and $52.50 \%$ of patients being classified at lower risk. Of the very small percent of patients placed in the higher-risk group, $13.03 \%$ of these would be predicted to have a readmission (Table 101). For those in the lower-risk group, $1.74 \%$ would be predicted to be readmitted.

\section{Total cost}

The best All IVs model from the knee data set identified several groups of patients whose particular combinations of IV states from the model S Tcb : Nrb Tcb (Table 76) would be expected to have higher total cost (Tcb). Considering all of these groups of patients together, $21.49 \%$ of the total patients (Table 102) were placed in the higher expected cost group, with an expected cost of \$20,907.88, higher (although not tested for 
statistical significance) than the average of the total knee data set $(\$ 18,502.55)$. For this same model, $21.86 \%$ of patients had a lower expected cost of $\$ 16,282.24$.

Table 102. Increased and Decreased Risk IV States for Total Cost (Tcb).

\begin{tabular}{|c|c|c|c|c|}
\hline \multicolumn{3}{|l|}{ Total Cost Binned (Tcb) } & & \\
\hline \multicolumn{3}{|l|}{ Knee (All IVs) } & & \\
\hline Model: S Tcb : Nrb Tcb & Freq & $\%$ of Cases & Ratio Average & Expected Value \\
\hline Increased Expected Cost IV States & 932 & $21.49 \%$ & 1.13 & $\$ 20,907.88$ \\
\hline Decreased Expected Cost IV States & 948 & $21.86 \%$ & 0.88 & $\$ 16,282.24$ \\
\hline No difference (by significance or frequency) & 2456 & $56.64 \%$ & & \\
\hline Total & 4336 & & & $\$ 18,502.55$ \\
\hline \multicolumn{3}{|l|}{ Hip (All IVs) } & & \\
\hline Model: L Tcb : Svb Tcb : Nrb Tcb & Freq & $\%$ of Cases & Ratio Average & Expected Value \\
\hline Increased Expected Cost IV States & 690 & $21.53 \%$ & 1.11 & $\$ 20,636.51$ \\
\hline Decreased Expected Cost IV States & 907 & $28.30 \%$ & 0.88 & $\$ 16,360.48$ \\
\hline No difference (by significance or frequency) & 1608 & $50.17 \%$ & & \\
\hline Total & 3205 & & & $\$ 18.591 .45$ \\
\hline
\end{tabular}

The best model for hip (L Tcb : Svb Tcb : Nrb Tcb) also identified groups of patients who would be expected to have total cost (Tcb) both higher and lower than the average of $\$ 18,591.45$. Of the hip patients, $21.53 \%$ were calculated to have an expected cost of $\$ 20,636.51$, while $28.30 \%$ would be expected to have a total cost of $\$ 16,360.48$ (Table 102).

\section{Methodological Considerations}

In addition to the clinical impacts, this project provides two primary methodological contributions. The first of these is the comparison of Reconstructability Analysis (the primary methodology of this project) to the more common approach of logistic regression. The second contribution is the demonstration of multiple techniques that exemplify and augment the use of RA for the analysis of biomedical data. 


\section{Relating RA to LR}

RA, as implemented in the Occam software package, was shown to produce equivalent results to LR models implemented in the R programming language, for those cases where the two methods overlap. This equivalency was verified with the measures Alpha (p-value), $\Delta$ df, $\Delta \mathrm{LR}, \Delta \mathrm{AIC}$, and odds ratios (OR) calculated from the RA model's conditional probability distributions. RA detected novel variables and interaction effects that were not found in the LR analysis. RA was demonstrated to provide a distinct and useful approach for searching for novel variables and interaction effects that resulted in stronger predictions. LR does not consider the full lattice of structures that RA does, nor does LR, as normally implemented in software, provide an approach for searching this lattice. The information-theoretic RA used in this research project generated an entropy reduction $(\% \Delta \mathrm{H})$, useful in interpreting the results, but not available with other methods.

The LR model augmented with interactions found by RA provided for an interesting test of the methods; it showed that non-hypothesized interaction effects discovered by RA offered additional predictive strength when added to LR models (Table 18). The models generated as the primary results of this project are therefore likely to be either equivalent or more predictive than an LR analysis could have generated. RA is useful as a standalone method and has the potential to generate more predictive models than is possible using the LR methodology. 


\section{Augmenting RA Occam Results}

This project supplemented Occam's standard outputs with existing nominal variable techniques that may be useful to researchers using the RA method in future analysis of biomedical data. The use of risk ratios, expected value calculations, tiered classifications of variables, and decision trees are all techniques that served to help make RA results visible and comprehensible.

\section{Tiered Classification System of Important Predicting Variables}

In this project, the importance of the IVs was assessed according to a multi-tiered classification system. This system allowed the identification and communication of important variables both within and across multiple models. RA yielded multiple models, which differed in how aggressively or conservatively they asserted predictive relations. While the models selected to look at in greater detail in the results section of this project were selected by the more conservative BIC criterion, the models and their variables that were selected by the less conservative AIC criterion are also relevant. While Tier 1 best predicting variables included only those from the best BIC models from All IVs, Tier 2 expanded and included variables detected as important predictors from the AIC selection.

\section{Decision Trees to Summarize Fit Results}

Each model's variables show the IVs and IV relationships that are important, but it is the model's internal structure, namely its conditional probability distribution (Occam's "fit" output), that allows the actual predictions of the model. These conditional probability distributions require some further analysis in order to determine the IV states of the model that are most important. In this research project, IV states with a frequency 
$\geq 10$ and a $p$-value $\leq 0.05$ were selected as thresholds, and IV states that did not meet these thresholds were removed from further analysis. Even with this reduction in the model IV states, the number of IVs, and the number of states for each of these IVs, resulted in fairly large tables, and the combinations of IV states that were important were not always readily comprehensible. Displaying combinations of important IV states in decision trees provides a visualization of results that is easily understood by a variety of audiences, allowing complex predictive models to offer descriptive insights from each model.

\section{Generating Continuous Predictions}

In this research project, continuous expected value predictions were generated for total cost with the b-systems approach (Zwick, Fusion, \& Wilmot). RA as programmed in Occam calculated probabilities of the DV in low-cost, mid-cost, and high-cost bins, conditioned on the model's IV states. These probabilities were used as weights on the expected value of each bin (the average value of each bin) to calculate the expected value for each of the IV states. Instead of using the bin with the highest probability to make a prediction, ignoring other bins whose probabilities, though smaller, may still not be negligible, this project used a weighted average which took into account how much the model predicted for the probability of each bin. Instead of picking one out of three bins for each of the IV states of the model, this method allowed for a finer-grained continuous prediction, with the ability to predict more accurately. Other augmentations of RA can be used to generate continuous predictions, which will be discussed in the Future Research 
section to follow. Such continuous predictions could in principle be compared to the prediction of regression methods.

\section{Risk Ratios}

Recall that each predictive model had an associated conditional probability distribution for all the DV states for each of the IV states that were observed. Each probability of an adverse DV state given a particular IV state was then divided by probability of the adverse outcome for the full sample. This calculated ratio conveyed the effect size, while the p-value for that IV state assessed the significance of this effect size, more precisely the statistical significance of the deviation of the IV conditional probabilities from the margins. This ratio allowed for the effect size of each predictive model to be compared across hip and knee data sets, for All IVs and Comorbidity IVs, and across each of the four DVs. The risk ratios from the individual model results were then averaged by the weighted frequency, thus allowing comprehensible and comparable average risk ratios for higher-risk and lower-risk IV states. Additionally, when multiplied by the margin of the overall sample, the risk ratio allowed for a new calculation of the expected rate of the outcome (Cp, SNF, and Re) and the expected value (Tcb) for the higher-risk and lower-risk IV states.

In this project, an effect size of $10 \%$ (a ratio $\geq 1.10$ or $\leq 0.90$ ) was considered to be a meaningful effect size. The selection of effect size depended on what was justifiable in the clinical and financial context of hip and knee replacement. At the time this research project was conducted, the largest payer of hip and knee replacements, Center for Medicare and Medicaid Services (CMS), had just implemented the first year of a 
five-year bundled payment model for hip and knee replacements. In this program, hospitals stand to gain or lose based on a percentage of CMS's contracted price (target price). In year one of this program, gains (upside risk) and losses (downside risk) are capped at $5 \%$, but will increase to $20 \%$ in the remainder of the program. At the same time that the risk is increased, the reimbursement prices will also be declining. This places hospitals at increasing risk of owing money back to CMS.

The following is a rough example of what the effect size looks like in terms of total cost. Looking at the higher-risk IV states (with the $10 \%$ or greater effect size) results in 324 hip patients at increased risk of higher expected total cost. Overall, the expected total cost for these 324 patients is $\$ 6,780,339$. If these same 324 patients were instead expected to have the average total cost, this would be approximately $\$ 6,023,629$ a difference of $\$ 756,709$, or about $\$ 2,335$ per patient. This difference is a real opportunity if care could be tailored for these patients so that costs for them could be reduced. If only half of the opportunity is realized in terms of cost savings, that is still about $\$ 1,167$ per patient. The 5\% reduction in payment (CMS pricing based on total average cost) is about $\$ 930$ in reduced payments, so this $\$ 1,167$ would more than offset the reduction of CMS reimbursement. The clinical impacts, one could argue, are even more meaningful. Complications and readmissions are certainly costly, but the effect on the patients can be profound. 


\section{Future Research \& Applications}

Future research should be performed in order to (a) address limitations of the current project, and (b) facilitate the real-world application of these models. There are at least three primary areas for future research that should be considered to address the limitations of this project: (1) The data used in this project must be updated according to new diagnosis coding standards and claims data availability; (2) data sets should be divided into training and test splits; and (3) additional RA techniques should be explored that may create more powerful analyses.

Potential applications of this research for hip and knee replacement surgery include enhancing value through measuring quality and implementing real-time risk prediction. Additional potential applications of the RA methodology used in this project are both desirable and feasible in clinical services beyond orthopedics, such as cardiovascular interventions.

\section{RA Analysis}

This project did not exhaust the RA methodological toolbox. Future research could use state-based RA modeling to create more powerful analyses. Additionally, other techniques for dealing with the continuous DV Total Cost should be explored, such as "K-systems" and "U-systems" analysis (Zwick, 2011b). Lastly, training and test splits of the data would allow for comparisons of sensitivity and specificity.

\section{State-based Models}

Taking only the most important predictor variables, state-based modeling would allow for the detection of interaction effects at a finer resolution. This ultra-fine-grained 
view may pick out subtle interaction effects that went undetected in the coarse and finegrained exploratory searches that were used in this project. It is possible that the statebased model will provide a larger reduction in uncertainty $(\% \Delta \mathrm{H})$ and better $\%$ correct than the best variable-based model with loops generated in this project.

$K$-systems and $U$-systems Analysis

This project derived continuous values for the binned DV Total Cost using expected value calculations, which is referred to as b-systems analysis in the literature (Zwick, 2011b). There are other RA techniques that are available for the analysis of continuous functions (i.e., total cost), such as k-systems and u-systems analysis. In k-systems analysis, continuous function values are treated as pseudo frequencies, whereas in u-systems they are treated as the expected value of a lottery (Zwick, 2011b).

\section{Training/Test Splits}

This project was exploratory in nature. Although an optimal confirmatory test of results found by exploratory modeling requires the availability of new data, a kind of confirmatory test is accomplished via the use of training and test splits of the data. The training portion of the data is used to find and fit the predictive models, and the test portion is used to validate these models. Ideally, multiple training/test splits should be used to obtain a probability distribution of test data outcomes, including but not limited to \%correct. This standard measure would allow easy comparisons of RA with other prediction methods. Test data can be used to calculate additional useful measures, such as sensitivity and specificity and their tradeoff in the ROC curve. 


\section{Binning}

In this project, binning (e.g., of the IVs age and surgeon volume and the DV total cost) was done, wherever possible, with equally sampled bins, which is mathematically optimal for prediction; this is as opposed to binning according to field-specific substantive conventions. While the use of three bins allows for detection of nonlinearities and a uniform distribution allows for optimal predicting power, other binning rules could be tested in future work. For example, when age was binned into three equal-samplesized bins, the age for enrollment onto Medicare was not selected as one of the bin boundaries. Binning according to the commonly used age groups in health outcomes research may change the predictions, which could be informative and could also have implications for healthcare policy.

\section{Enhancing the Data}

\section{Updating Coded Data}

The primary procedure codes used in this project—both those indicating hip or knee replacement procedures and those indicating patient comorbidities — were constructed from ICD-9 diagnosis codes. Effective October 2015, new ICD-10 coding standards were introduced. These new coding standards are not simply an updated version of the previous coding schema. There are more than ten times as many procedure codes and five times as many diagnosis codes in ICD-10 than in its ICD-9 predecessor. This update allows for a much more detailed system of coding. A single diagnosis code in ICD-9 may now be broken down into much more specific diagnosis codes. Most certainly this finer resolution of coding will provide for new discoveries as to the most predictive 
patient comorbidity variables. While dual coding for ICD-9 and ICD-10 is still occurring in order for existing initiatives to be sustained, there is no doubt that ICD-9 coding will soon be phased out completely.

\section{Social Determinants of Health}

Social determinants of health, such as poverty, access to healthcare services, and racism, may be important predictors of differences in health outcomes. Widespread collection and measurement of social determinants would need to be initiated in order to have consistent and available data for predictive models.

\section{Adding Post-Discharge Data}

Additional enrichment to the data could be performed to enhance the dependent variables used in this project. One limitation in this project is in the DV Total Cost. The DV Total Cost only captures costs associated with the inpatient procedure, and does not include post-discharge costs. These post-discharge costs may be incurred by the utilization of a skilled nursing facility (SNF) as well as by Readmissions and other postdischarge services (i.e., outpatient therapy, home health visits, etc.). Payer claims data would allow for Total Cost to extend beyond the inpatient stay, and beyond that, a DV representative of a 90-day episode could be constructed. Additionally, the DV Complication only captured complications that occurred within the inpatient stay, and does not include events post-discharge. Joint replacement patients may do well in the hospital but suffer complications post-discharge that indicate poor quality and incur additional costs. Bundled payments (such as the 90-day CJR model) will increasingly 
drive healthcare systems to be able to capture costs and outcomes that extend beyond the initial inpatient surgical encounter.

As health systems form Accountable Care Organizations (ACOs) and as CMS reimbursement models increase, access to claims data is more feasible than was the case historically. ACOs and CMS have rich data sources with very detailed claims data. Claims data provides an effective way to look at the utilization in services and could be used to create more meaningful and stronger predictions. At this time, however, detailed claims data is not available for all patients. Additionally, uncertainty about how claims data can be used often results in restrictions that block the ACO and CMS data from being part of the healthcare organization's larger data repositories. The propensity for healthcare systems to treat analysis of data as a research effort, rather than a quality improvement and patient care effort, prevents most analytical efforts — and certainly anything referred to as "exploratory" analysis - from occurring. However, as the terms "big data" and "predictive analytics" become a more mainstream part of the vocabulary of healthcare delivery systems, ACO and CMS claims data will have the potential to become part of the larger data environment with greater access for use in predictive analytics.

\section{Adding the Delivery System Data Domain}

Currently, the handful of predictive analytics tools that exist use only patient comorbidity IVs. This project also utilized delivery system variables, but only a subset of the potential variables that may impact predictions. The small number of delivery system IVs that were used in this project turned out to be very important predictors in a number 
of the models. In order to enhance value through better predictions, healthcare systems will need to look at the processes of care, including the clinicians, locations, medications, implants, and other supplies used in those processes as potentially predictive of patient outcomes and cost.

\section{Reimbursement \& Allocation of Payments}

Hip and knee replacements are the first set of procedures in the bundled payment Comprehensive Care for Joint Replacement (CJR) model. As discussed in the introduction to this project, this model includes fixed payments to the delivery system based on DRG. If these payments exceed the cost of the episode, the leftover dollars are eligible to be paid out in collaborator "cost savings" or "gainsharing" payments. In these collaborator agreements, it is often the surgeon who is identified as the collaborator, and how the payments are allocated is determined by the hospital. Such payments can serve as an incentive to reward surgeons not only for low-cost care, but for quality outcomes as well. Incorporating risk into the projection of expected outcomes can help drive better care without penalizing surgeons for taking on sicker patients. As delivery systems determine how to allocate savings among surgeon collaborators under the CJR model, using a quality measure (discussed below) that adequately accounts for predicted patient risk would be worth considering. 


\section{Translating Predictions into Value}

\section{Real-Time Risk Prediction}

In the introduction to this project, a primary justification for this research was that predictive models have utility both for retrospectively predicting risks and outcomes for quality measurement, and_-potentially_also for real-time prediction.

Many total joint replacement patients prefer to be in and out of the hospital as quickly as possible, and hospitals (and outpatient surgery centers) are now marketing short-stay joint procedures as a way to attract healthy patients. However, this preference must be considered alongside patient risk and clinical appropriateness, and the risk algorithms for determining which patients may qualify for these short stays are lacking. In other instances, healthy patients who may qualify as short-stay candidates request to stay in the hospital as long as possible with expectations to be discharged to a skilled nursing facility. Under a fee-for-service reimbursement model, patients and providers had no incentive to guard against patient preference as the primary driver of length of stay or discharge setting. As fee-for-service comes to an end in joint replacement, patient preference or physician practice patterns alone can no longer be the primary driver of appropriate care. Assessing patient risk in real time could in principle augment clinical decision-making and provide additional evidence supporting a short-stay care pathway for low-risk patients, or a more resource-intensive care pathway for high-risk patients. Furthermore, increasing the education of patients about their individual risks can help set expectations about their individualized care pathways. 
Retrospective analysis is much simpler than developing and operationalizing a near-real-time risk-prediction solution in a healthcare organization. To make predictions in near real time, one would need access to or integration with an electronic health record (EHR) system or another data aggregation system overlaying the EHR. This additional application layer would need to access the patient's clinical data and transform the data into a structured format according to predefined rules similar to the procedures used to create the data sets used in this project. Predictive models would have to be updated and calibrated on a continuing basis, to improve the models based on their performance in prediction and to keep up with clinical coding schema changes.

Not only would models need to be integrated with the clinical electronic health record system, but the model predictions would need to be accessible within the EHR in order for the clinicians at the point of care to utilize the output to support real-time decision making. Recent advances in machine learning and AI techniques have increased focus on the development of the algorithms but have given less attention to how predictions made by algorithms might inform patient care in real-time clinical work streams. Great predictions do not help if no action is taken.

Integrating predictions into the everyday workflows of clinical staff is much more than a technical feat. It is an organizational undertaking that would require the buy-in of health system leadership and of the clinical teams. The development of predictive models is only a first step. Few health systems currently use predictive analytics at scale to influence health care delivery, and strategies that successfully implement predictive risk algorithms into clinical practice must still be identified (Parikh, Kakad \& Bates, 2016). 


\section{Measuring Quality \& Outcomes}

With value defined as outcomes achieved per dollar spent, an increasing focus of concern will be the optimization of that ratio. Deploying predictive analytics at the point of care can help improve this ratio by focusing costly resources on higher-risk patients. Tailoring patient care with increased precision thus has the potential not only to lower the overall cost of care, but to improve outcomes by ensuring that higher-risk patients are provided the extra care necessary to ensure good outcomes. In addition to optimizing care in real time, the demonstration of value requires the measurement of outcomes.

Adequately measuring the quality of the outcome achieved is essential both for fair measurement of hospital or physician quality and for identifying the causes of variation in care, both of which will be required in order to strategically focus quality improvement initiatives. A widely-accepted measurement of quality across healthcare providers is an observed versus expected ratio. The denominator of this ratio is the sum of predicted risk of a given outcome while the numerator is the actual observed occurrences of this outcome. In the field of cardiac surgery, the Society of Thoracic Surgeons (STS) Risk Score (Shahian et al., 2009) generates an O/E ratio for mortality in addition to a number of specific complications. The resulting scores from the O/E ratios are used as measures of quality both at a hospital and a surgeon level. For example, if Dr. A's patients were predicted to have a higher risk of complications given their comorbidity IVs, and subsequently the observed complications were calculated, then an observed versus expected ratio could be constructed and used as a measure of quality. If the cohort of patients had fewer complications than predicted, then the ratio would be less than one, and this distance from one could be considered a measure indicating higher quality 
outcomes. If the ratio is greater than one, then this would indicate surgeon performance was worse than expected. The result of this observed versus predicted ratio can then be assessed across physicians in a relatively fair, "apples-to-apples" comparison. Similar to cardiac surgery, joint replacement surgeons are considered the most accountable for the quality and outcomes of the joint replacement procedure. However, unlike cardiac surgery, there is no widely accepted observed versus expected risk score in joint replacement.

\section{Value Beyond Orthopedics}

A primary justification for this project was that better predictions, utilized for quality measurement and real-time predictive analytics, could enhance value in healthcare. This project demonstrated that Reconstructability Analysis (RA) can create useful models of outcomes in hip and knee replacements. Hip and knee replacements are the first procedures mandated under a bundled payment model by CMS. Other payers are coming to the payment reform table as well, and the list of conditions and procedures that CMS and other payers will be transitioning to innovative reimbursement models is extensive. In fact, CMS has set a goal, for both public and private payers, to shift $80 \%$ of their populations into value-based alternative payment models in the next five years (Rajkumar, Conway \& Tavenner, 2014). The drive to achieve greater value across multiple clinical domains is now an imperative for healthcare systems.

CMS has passed legislation for two cardiac bundled payments set to launch in 2018 which will cover Acute Myocardial Infarction (AMI) and Coronary Artery Bypass Graft (CABG) procedures (Song \& Blumenthal, 2016). Similar to joint replacement 
procedures, these cardiac procedures have high variation in cost and outcomes. Unlike the field of orthopedics, the cardiovascular field has been collecting data and measuring quality and outcomes utilizing risk models for decades. In fact, cardiovascular procedures are some of the most well studied procedures, with highly validated risk models. The Society of Thoracic Surgeons' (STS) Cardiac Surgery Risk Model has been developed over a 20-year period, and is continuously updated based on STS registry data (Shahian et al., 2009). The data that is used for this model is manually abstracted from patient charts by clinicians and hand-entered into a certified STS database. This curated registry data is then submitted to the national STS registry, and model updates are performed. Standard Logistic Regression (LR) has been the primary method for STS model development, with the most recent updates to the model using backwards stepwise LR for feature selection in order to determine predictor variables for model updates. The updated STS model is considered to have excellent performance, but it is certainly plausible the performance could be improved.

Literature in cardiac risk prediction confirms LR to be the standard approach within the broader cardiac field (Nilsson, Algotsson, Höglund, Lührs \& Brandt, 2006; Thalji, Suri, Greason \& Schaff, 2014). However, researchers view more recent techniques such as Neural Networks (NNs) as holding promise. Nilsson et al., (2006) discuss NNs as a method that could improve risk prediction given their capacity to model complex, nonlinear relationships, but concede that only a few studies have been done in this area and that further investigation is required. As discussed in the literature review of this project, NNs are promising, but their "black box" nature results in models that lack explainability. This lack of transparency can pose a problem with clinician buy-in during 
implementation in a clinical setting. Perhaps the need for explainability will diminish over time as NNs prove their worth, and trust in these models increases.

Reconstructability Analysis (RA) has both the capacity to model complex, nonlinear relationships, and the ability to produce models that are transparent and intuitive to understand. This project showed that RA was able to find a more predictive model than stepwise LR, and included additional predictor variables and interaction terms not detected using stepwise LR. It would be of great interest to cardiac clinicians and researchers if RA could improve on the STS Risk Score predictions. If RA can provide new insights with stronger predictions in the cardiovascular domain, it is likely the methodology would be able to add value across the full spectrum of clinical service lines.

The field of cardiovascular risk prediction may benefit from additional analytical approaches used in this project, beyond potentially detecting novel predictor variables and interaction effects. This project demonstrated the importance of delivery system IVs in predicting outcomes. Currently, the risk prediction efforts in the cardiovascular domain limit predictors to patient-related comorbidity IVs. Additionally, the use of risk ratios would be intuitive and meaningful to clinicians in understanding — beyond simply the significance of the prediction - the magnitude of the expected effect. The use of tiered analysis of predictors may reveal common predictors across cardiac and joint replacement surgeries and allow for a broader implementation of predictive analytics in clinical care and the measurement of quality across clinical domains.

Enhancing value through better predictions is an increasing imperative across multiple clinical domains. As demonstrated in this project, Reconstructability Analysis is an approach that may strengthen or augment existing predictions and even perhaps 
replace existing methods. With risk and outcomes adequately predicted, areas for potential improvement become clearer, and focused changes can be made to drive improvements in patient care. Better predictions, such as those resulting from the Reconstructability Analysis methodology, can thus support improvement in value-better outcomes at a lower cost. As reimbursement increasingly evolves into value-based programs, understanding the outcomes achieved and customizing patient care to reduce unnecessary costs while improving outcomes will be active areas for clinicians, healthcare administrators, researchers, and data scientists for many years to come. 


\section{References}

Amarasingham, R. (2012). Applying Data Analytics And Information Exchange To Improve Care For Patients. Health Affairs, 31(12), 2785-2786.

http://doi.org/10.1377/hlthaff.2012.1114

Andrew, J. G., Palan, J., Kurup, H. V., Gibson, P., Murray, D. W., \& Beard, D. J. (2008). Obesity in total hip replacement. Journal of Bone \& Joint Surgery, British Volume, 90-B(4), 424-429. http://doi.org/10.1302/0301-620X.90B4.20522

Ash AS, E. R. (2012). Risk-adjusted payment and performance assessment for primary care. Medical Care, 50(8), 643-53.

Ashby, R. (1964). Constraint analysis of many-dimensional relations. General Systems Yearbook, 9, 99-105.

Barsoum, W. K., Murray, T. G., Klika, A. K., Green, K., Miniaci, S. L., Wells, B. J., \& Kattan, M. W. (2010). Predicting Patient Discharge Disposition After Total Joint Arthroplasty in the United States. The Journal of Arthroplasty, 25(6), 885-892. http://doi.org/10.1016/j.arth.2009.06.022

Bates, D. W., Saria, S., Ohno-Machado, L., Shah, A., \& Escobar, G. (2014). Big Data In Health Care: Using Analytics To Identify And Manage High-Risk And High-Cost Patients. Health Affairs, 33(7), 1123-1131. http://doi.org/10.1377/hlthaff.2014.0041

Bellows, J., Patel, S., \& Young, S. S. (2014). Use of IndiGO individualized clinical guidelines in primary care. Journal of the American Medical Informatics Association, 21(3), 432-437. http://doi.org/10.1136/amiajnl-2012-001595

Berbari, E. F., Hanssen, A. D., Duffy, M. C., Steckelberg, J. M., Ilstrup, D. M., Harmsen, W. S., \& Osmon, D. R. (1998). Risk Factors for Prosthetic Joint Infection: CaseControl Study. Clinical Infectious Diseases, 27(5), 1247-1254.

http://doi.org/10.1086/514991

Berbari, E. F., Osmon, D. R., Lahr, B., Eckel-Passow, J. E., Tsaras, G., Hanssen, A. D., ... Thompson, R. (2012). The Mayo prosthetic joint infection risk score: implication for surgical site infection reporting and risk stratification. Infection Control and Hospital Epidemiology: The Official Journal of the Society of Hospital Epidemiologists of America, 33(8), 774-781. http://doi.org/10.1086/666641

Bjorgul, K., Novicoff, W. M., \& Saleh, K. J. (2010). Evaluating comorbidities in total hip and knee arthroplasty: available instruments. Journal of Orthopaedics and Traumatology, 11(4), 203-209. http://doi.org/10.1007/s10195-010-0115-x 
Bolognesi, M. P., Marchant, M. H., Viens, N. A., Cook, C., Pietrobon, R., \& Vail, T. P. (2008). The Impact of Diabetes on Perioperative Patient Outcomes After Total Hip and Total Knee Arthroplasty in the United States. The Journal of Arthroplasty, 23(6), 92-98. http://doi.org/10.1016/j.arth.2008.05.012

Bozic, K. J., Ong, K., Lau, E., Berry, D. J., Vail, T. P., Kurtz, S. M., \& Rubash, H. E. (2012). Estimating Risk in Medicare Patients With THA: An Electronic Risk Calculator for Periprosthetic Joint Infection and Mortality. Clinical Orthopaedics and Related Research®, 471(2), 574-583. http://doi.org/10.1007/s11999-0122605-z

Bozic, K. J., Smith, A. R., \& Mauerhan, D. R. (2007). Pay-For-Performance in Orthopedics: Implications for Clinical Practice. The Journal of Arthroplasty, 22(6, Supplement), 8-12. http://doi.org/10.1016/j.arth.2007.04.015

Bozic, K. J., Wagie, A., Naessens, J. M., Berry, D. J., \& Rubash, H. E. (2006). Predictors of Discharge to an Inpatient Extended Care Facility After Total Hip or Knee Arthroplasty. The Journal of Arthroplasty, 21(6), 151-156. http://doi.org/10.1016/j.arth.2006.04.015

Broekstra, G. (1979). Nonprobabilistic constraint analysis and a two-stage approximation method of structure identification. In Proceedings of teh 23rd Annual SGSR Meeting, Houston.

Cangur, O. (2009). Modeling Subprime Mortgage Delinqency, Default, Prepayment and Loss.

Caracciolo, B., \& Giaquinto, S. (2005). Determinants of the subjective functional outcome of total joint arthroplasty. Archives of Gerontology \& Geriatrics, 41(2), 169-176. http://doi.org/10.1016/j.archger.2005.01.005

Caring for High-Need, High-Cost Patients - An Urgent Priority — NEJM. (n.d.). Retrieved May 6, 2017, from http://www.nejm.org/doi/full/10.1056/NEJMp1608511\#t=article

Carletti, R. (2004). A Study of the Relationship Between Education, Literacy, and Health.

Cavallo, R. E. (1979). The role of systems methodology in social science research.

Cellier, F. E., \& Yandell, D. W. (1987). SAPS-II: A NEW IMPLEMENTATION OF THE SYSTEMS APPROACH PROBLEM SOLVER. International Journal of General Systems International Journal of General Systems, 13(4), 307-322. 
Centers for Medicare \& Medicaid Services. Comprehensive care for joint replacement model. (2015).

Centers for Medicare \& Medicaid Services (CMS), HHS. (2011). Medicare program; Medicare Shared Savings Program: Accountable Care Organizations. Final rule. Federal Register, 76(212), 67802-67990. Retrieved from http://www.ncbi.nlm.nih.gov/pubmed/22046633

Chambless, B., Lendaris, G. G., \& Zwick, M. (2001). An information theoretic methodology for prestructuring neural networks (Vol. 1, pp. 365-370 vol.1). http://doi.org/10.1109/IJCNN.2001.939047

Clague, J. E., Craddock, E., Andrew, G., Horan, M. A., \& Pendleton, N. (2002).

Predictors of outcome following hip fracture. Admission time predicts length of stay and in-hospital mortality. Injury, 33(1), 1-6. Retrieved from http://www.injuryjournal.com/article/S0020-1383(01)00142-5/abstract

Conant, R. C. (1981). Set-Theoretic Structure Modeling. International Journal of General Systems, 7, 93-107.

Conant, R. C. (1988). EXTENDED DEPENDENCY ANALYSIS OF LARGE SYSTEMS*. International Journal of General Systems International Journal of General Systems, 14(2), 97-123.

Cram P, L. X. (2011). CLinical characteristics and outcomes of medicare patients undergoing total hip arthroplasty, 1991-2008. JAMA, 305(15), 1560-1567. http://doi.org/10.1001/jama.2011.478

Dall, G. F., Ohly, N. E., Ballantyne, J. A., \& Brenkel, I. J. (2009). The influence of preoperative factors on the length of in-patient stay following primary total hip replacement for osteoarthritis A MULTIVARIATE ANALYSIS OF 2302 PATIENTS. Journal of Bone \& Joint Surgery, British Volume, 91-B(4), 434-440. http://doi.org/10.1302/0301-620X.91B4.21505

Dauty, M., Schmitt, X., Menu, P., Rousseau, B., \& Dubois, C. (2012). Using the Risk Assessment and Predictor Tool (RAPT) for patients after total knee replacement surgery. Annals of Physical and Rehabilitation Medicine, 55(1), 4-15. http://doi.org/10.1016/j.rehab.2011.10.006

Doyle, J., Graves, J., \& Gruber, J. (2015). Uncovering Waste in U.S. Healthcare. Retrieved from http://www.nber.org/papers/w21050 
Eddy, D. M., Adler, J., \& Morris, M. (2012). The "Global Outcomes Score”: A Quality Measure, Based On Health Outcomes, That Compares Current Care To A Target Level Of Care. Health Affairs, 31(11), 2441-2450. http://doi.org/10.1377/hlthaff.2011.1274

Everhart, J. S., Altneu, E., \& Calhoun, J. H. (2013). Medical Comorbidities Are Independent Preoperative Risk Factors for Surgical Infection After Total Joint Arthroplasty. Clinical Orthopaedics and Related Research. http://doi.org/10.1007/s11999-013-2923-9

Fehring, T. K., Odum, S. M., Troyer, J. L., Iorio, R., Kurtz, S. M., \& Lau, E. C. (2010). Joint Replacement Access in 2016: A Supply Side Crisis. The Journal of Arthroplasty, 25(8), 1175-1181. http://doi.org/10.1016/j.arth.2010.07.025

Fisher, E. S. (2003). The Implications of Regional Variations in Medicare Spending. Part 1: The Content, Quality, and Accessibility of Care. Annals of Internal Medicine, 138(4), 273. http://doi.org/10.7326/0003-4819-138-4-200302180-00006

Gandhi, R., Dhotar, H., Razak, F., Tso, P., Davey, J. R., \& Mahomed, N. N. (2010). Predicting the longer term outcomes of total knee arthroplasty. Knee, 17(1), 15-18. http://doi.org/10.1016/j.knee.2009.06.003

Gholson, J. J., Pugely, A. J., Bedard, N. A., Duchman, K. R., Anthony, C. A., \& Callaghan, J. J. (2016). Can We Predict Discharge Status After Total Joint Arthroplasty? A Calculator to Predict Home Discharge. The Journal of Arthroplasty, 31(12), 2705-2709. http://doi.org/10.1016/j.arth.2016.08.010

Goroll AH, \& Schoenbaum SC. (2012). Payment reform for primary care within the accountable care organization: A critical issue for health system reform. JAMA, 308(6), 577-578. http://doi.org/10.1001/jama.2012.8696

Head, S. J., Osnabrugge, R. L. J., Howell, N. J., Freemantle, N., Bridgewater, B., Pagano, D., \& Kappetein, A. P. (2013). A systematic review of risk prediction in adult cardiac surgery: considerations for future model development. European Journal of Cardio-Thoracic Surgery: Official Journal of the European Association for CardioThoracic Surgery, 43(5), e121-129. http://doi.org/10.1093/ejcts/ezt044

Hines Al, Barrett M1, Jiang Hj, \& Steiner Ca. (2011). Conditions With the Largest Number of Adult Hospital Readmissions by Payer, 2011: Statistical Brief \#172. Retrieved from http://europepmc.org/abstract/med/24901179 
HIPAA. (2009, September). HIPAA "Protected Health Information": What Does PHI Include? Retrieved from https://www.hipaa.com/hipaa-protected-healthinformation-what-does-phi-include/

Hosmer, D.W, Lemeshow, S. (2005). Applied logistic regression (second edi). John Wiley \& Sons Inc.

Jacobs JP, J. M. (2009). Stratification of complexity improves the utility and accuracy of outcomes analysis in a Multi-Institutional Congenital Heart Surgery Database: Application of the Risk Adjustment in Congenital Heart Surgery (RACHS-1) and Aristotle Systems in the Society o. Pediatric Cardiology, 30(8), 1117-30.

Jafari, S. M., Huang, R., Joshi, A., Parvizi, J., \& Hozack, W. J. (2010). Renal Impairment Following Total Joint Arthroplasty: Who Is at Risk? The Journal of Arthroplasty, 25(6, Supplement), 49-53.e2. http://doi.org/10.1016/j.arth.2010.04.008

Jain, N. B., Guller, U., Pietrobon, R., Bond, T. K., \& Higgins, L. D. (2005). Comorbidities Increase Complication Rates in Patients Having Arthroplasty. Clinical Orthopaedics and Related Research, \&NA;(435), 232-238. http://doi.org/10.1097/01.blo.0000156479.97488.a2

Jones, B. (1985). Reconstructability analysis for general functions. International Journal of General Systems, 11, 133-142.

Jones, B. (1989). A PROGRAM FOR RECONSTRUCTABILUY ANALYSIS. International Journal of General Systems, 15(3), 199-205. http://doi.org/10.1080/03081078908935045

Klir, G. (1976). IDENTIFICATION OF GENERATIVE STRUCTURES IN EMPIRICAL DATA $\uparrow$. Int. J. of General Systems International Journal of General Systems, 3(2), 89-104.

Kramer, P., Westaway, S. K., Zwick, M., \& Shervais, S. (2012). Reconstructability analysis of genetic loci associated with Alzheimer disease. In The 6th International Conference on Soft Computing and Intelligent Systems, and The 13th International Symposium on Advanced Intelligence Systems (pp. 2104-2110). Ieee. http://doi.org/10.1109/SCIS-ISIS.2012.6505196

Krippendorff, K. (2009). No TitleRoss Ashby's information theory: a bit of history, some solutions to problems, and what we face today. International Journal of General Systems, 38(2), 189-212. 
Krippendorff, K. (1981). An algorithm for identifying structural models of multivariate data. International Journal of General Systems, 7(1), 63-79.

Kurtz S, O. K. (2007). Projections of primary and revision hip and knee arthroplasty in the United States from 2005 to 2030. The Journal of Bone and Joint Surgery. American Volume, 89(4), 780-5.

Lawrence, R. C., Helmick, C. G., Arnett, F. C., Deyo, R. A., Felson, D. T., Giannini, E. H., ... Hunder, G. G. (1998). Estimates of the Prevalence of Arthritis and Selected Musculoskeletal Disorders in the United States. ARTHRITIS AND RHEUMATISM ATLANTA-, 41(5), 778-799.

Lippmann, R. P., Kukolich, L., \& Shahian, D. (1995). Predicting the Risk of Complications in Coronary Artery Bypass Operations Using Neural Networks. Advances in Neural Information Processing Systems., (7), 1055.

Lippmann RP, S. D. (1997). Coronary artery bypass risk prediction using neural networks. The Annals of Thoracic Surgery, 63(6), 1635-43.

Lorenzoni, L., Belloni, A., \& Sassi, F. (2014). Health-care expenditure and health policy in the USA versus other high-spending OECD countries. The Lancet, 384(9937), 83-92. http://doi.org/10.1016/S0140-6736(14)60571-7

Luft, H. S. (2009). Economic Incentives to Promote Innovation in Healthcare Delivery. Clinical Orthopaedics and Related Research®, 467(10), 2497-2505. http://doi.org/10.1007/s11999-009-0930-7

Maciejewski, M. L., \& Liu, C.-F. (2005). The Performance of Administrative and SelfReported Measures for Risk Adjustment of Veterans Affairs Expenditures. Health Services Research, 40(3), 887-904.

Md, C. A. H., Karim Elsharkawy MD, M., Ms, A. K. K., Bs, M. B., \& Md, W. K. B. (2011). 2010 Mid-America Orthopaedic Association Physician in Training Award: Predictors of Early Adverse Outcomes after Knee and Hip Arthroplasty in Geriatric Patients. Clinical Orthopaedics and Related Research®, 469(5), 1391-1400. http://doi.org/10.1007/s11999-011-1804-3

Medicare, C. for. (2016, December). National Health Accounts Historical. Retrieved May 7, 2017, from https://www.cms.gov/Research-Statistics-Data-and-Systems/ Statistics-Trends-and-Reports/NationalHealthExpendData/ NationalHealthAccountsHistorical.html 
Meding, J. B. (2007). The Prescreening History and Physical in Elective Total Joint Arthroplasty. The Journal of Arthroplasty., 22(2), 21.

Memtsoudis, S. G., González Della Valle, A., Besculides, M. C., Esposito, M., Koulouvaris, P., \& Salvati, E. A. (2010). Risk Factors for Perioperative Mortality After Lower Extremity Arthroplasty: A Population-Based Study of 6,901,324 Patient Discharges. The Journal of Arthroplasty, 25(1), 19-26. http://doi.org/10.1016/j.arth.2008.11.010

Memtsoudis, S. G., Ma, Y., Chiu, Y. L., Walz, J. M., Voswinckel, R., \& Mazumdar, M. (2010). Perioperative Mortality in Patients with Pulmonary Hypertension Undergoing Major Joint Replacement. Anesthesia \& Analgesia, 111(5), 1110-1116. http://doi.org/10.1213/ANE.0b013e3181f43149

Miller, D. C., Gust, C., Dimick, J. B., Birkmeyer, N., Skinner, J., \& Birkmeyer, J. D. (2011). Large Variations In Medicare Payments For Surgery Highlight Savings Potential From Bundled Payment Programs. Health Affairs, 30(11), 2107-2115. http://doi.org/10.1377/hlthaff.2011.0783

Mist, S. D. (2007). Prediction of Traditional Chinese Medicine from Psychosocial Questionnaires.

Munin MC, K. C. (1995). Predicting discharge outcome after elective hip and knee arthroplasty. American Journal of Physical Medicine \& Rehabilitation / Association of Academic Physiatrists, 74(4).

Nichols, L. M., \& O'Malley, A. S. (2006). Hospital payment systems: will payers like the future better than the past? Health Affairs (Project Hope), 25(1), 81-93. http://doi.org/10.1377/hlthaff.25.1.81

Nilsson, J., Algotsson, L., Höglund, P., Lührs, C., \& Brandt, J. (2006). Comparison of 19 pre-operative risk stratification models in open-heart surgery. European Heart Journal, 27(7), 867-874. http://doi.org/10.1093/eurheartj/ehi720

Obama, B. (2016). United States Health Care Reform: Progress to Date and Next Steps. JAMA, 316(5), 525-532. http://doi.org/10.1001/jama.2016.9797

Oldmeadow, L. B., McBurney, H., \& Robertson, V. J. (2003). Predicting risk of extended inpatient rehabilitation after hip or knee arthroplasty. The Journal of Arthroplasty, 18(6), 775-779. http://doi.org/10.1016/S0883-5403(03)00151-7 
Parikh, R. B., Kakad, M., \& Bates, D. W. (2016). Integrating Predictive Analytics Into High-Value Care: The Dawn of Precision Delivery. JAMA, 315(7), 651-652. http://doi.org/10.1001/jama.2015.19417

Patient Protection and Affordable Care Act of 2010. (2010). Public Law 111, 48.

Polanczyk, C. A., Marcantonio, E., Goldman, L., Rohde, L. E. P., Orav, J., Mangione, C. M., \& Lee, T. H. (2001). Impact of Age on Perioperative Complications and Length of Stay in Patients Undergoing Noncardiac Surgery. Annals of Internal Medicine, 134(8), 637-643. http://doi.org/10.7326/0003-4819-134-8-200104170-00008

Porter, M. E. (2010). What Is Value in Health Care? New England Journal of Medicine, 363(26), 2477-2481. http://doi.org/10.1056/NEJMp1011024

Porter, M. E., \& Teisberg, E. O. (2006). Redefining Health Care: Creating Value-based Competition on Results. Harvard Business Press. Retrieved from https://books.google.com/books?id=Kp5fCkAzzS8C

Powers, B., \& Chaguturu, S. (2016). Not All High-Risk Populations Are the Same. New England Journal of Medicine, Catalyst.

Pulido L, P. J. (2008). In hospital complications after total joint arthroplasty. The Journal of Arthroplasty, 23(6), 139-45.

R. Core Team. (2016). R: A Language and Environment for Statistical Computing. Vienna, Austria: R Foundation for Statistical Computing; 2014. R Foundation for Statistical Computing.

Radcliff, K. E., Orozco, F. R., Quinones, D., Rhoades, D., Sidhu, G. S., \& Ong, A. C. (2012). Preoperative Risk Stratification Reduces the Incidence of Perioperative Complications After Total Knee Arthroplasty. The Journal of Arthroplasty, 27(8, Supplement), 77-80.e8. http://doi.org/10.1016/j.arth.2012.03.026

Rajkumar, R., Conway, P. H., \& Tavenner, M. (2014). CMS—Engaging Multiple Payers in Payment Reform. JAMA, 311(19), 1967-1968. http://doi.org/10.1001/jama.2014.3703

Romine, L. B., May, R. G., Taylor, H. D., \& Chimento, G. F. (2013). Accuracy and Clinical Utility of a Peri-Operative Risk Calculator for Total Knee Arthroplasty. The Journal of Arthroplasty, 28(3), 445-448. http://doi.org/10.1016/j.arth.2012.08.014 
Rozell, J. C., Courtney, P. M., Dattilo, J. R., Wu, C. H., \& Lee, G.-C. (2016). Should All Patients Be Included in Alternative Payment Models for Primary Total Hip Arthroplasty and Total Knee Arthroplasty? The Journal of Arthroplasty, 31(9), 4549. http://doi.org/10.1016/j.arth.2016.03.020

Schroer WC, C. G. (2008). Effects of increased surgical volume on total knee arthroplasty complications. The Journal of Arthroplasty, 23(6), 61-7.

Shahian, D. M., O’Brien, S. M., Filardo, G., Ferraris, V. A., Haan, C. K., Rich, J. B., ... Anderson, R. P. (2009). The Society of Thoracic Surgeons 2008 Cardiac Surgery Risk Models: Part 1 - Coronary Artery Bypass Grafting Surgery. The Annals of Thoracic Surgery, 88(1), S2-S22. http://doi.org/10.1016/j.athoracsur.2009.05.053

Shannon, C. E. (1948). A Mathematical Theory of Communication. The Bell System Technical Journal, 27, 379-423 and 623-656.

Shervais, S., Kramer, P. L., Westaway, S. K., Cox, N. J., \& Zwick, M. (2010). Reconstructability Analysis as a Tool for Identifying Gene-Gene Interactions in Studies of Human Diseases. Statistical Applications in Genetics \& Molecular Biology, 9(1), 1-25. Retrieved from http://stats.lib.pdx.edu/proxy.php?url=http://search.ebscohost.com/login.aspx?direct $=$ true $\& \mathrm{db}=\mathrm{a} 9 \mathrm{~h} \& \mathrm{AN}=48991209 \&$ site $=$ ehost-live

Song, Z., \& Blumenthal, D. M. (2016). Expanding Payment Reform in Medicare: The Cardiology Episode-Based Payment Model. JAMA, 316(19), 1973-1974. http://doi.org/10.1001/jama.2016.16146

Suleiman, L. I., Ortega, G., Ong'uti, S. K., Gonzalez, D. O., Tran, D. D., Onyike, A., ... Fullum, T. M. (2012). Does BMI affect perioperative complications following total knee and hip arthroplasty? The Journal of Surgical Research, 174(1), 7-11. http://doi.org/10.1016/j.jss.2011.05.057

Thalji, N. M., Suri, R. M., Greason, K. L., \& Schaff, H. V. (2014). Risk assessment methods for cardiac surgery and intervention. Nature Reviews Cardiology, 11(12), 704-714. http://doi.org/10.1038/nrcardio.2014.136

Tomek, I. M., Sabel, A. L., Froimson, M. I., Muschler, G., Jevsevar, D. S., Koenig, K. M., ... Weinstein, J. N. (2012). A Collaborative Of Leading Health Systems Finds Wide Variations In Total Knee Replacement Delivery And Takes Steps To Improve Value. Health Affairs, 31(6), 1329-1338. http://doi.org/10.1377/hlthaff.2011.0935 
Tu JV, W. M. (1998). Predicting mortality after coronary artery bypass surgery: what do artificial neural networks learn? The Steering Committee of the Cardiac Care Network of Ontario. Medical Decision Making : An International Journal of the Society for Medical Decision Making, 18(2).

Weiner, J. P., Trish, E., Abrams, C., \& Lemke, K. (2012). Adjusting For Risk Selection In State Health Insurance Exchanges Will Be Critically Important And Feasible, But Not Easy. Health Affairs, 31(2), 306-315. http://doi.org/10.1377/hlthaff.2011.0420

Willett, K., \& Zwick, M. (2004). A software architecture for reconstructability analysis. Kybernetes: The International Journal of Systems \& Cybernetics, 33(5/6), 9971008.

Zwick, M. (2000). OCCAM: organization complexity computation and modeling. Portland State University Systems Science Program Internal Document.

Zwick, M. (2001). Wholes and parts in general systems methodology. In The character concept in evolutionary biology (pp. 237-256).

Zwick, M. (2004). An overview of reconstructability analysis. Kybernetes: The International Journal of Systems \& Cybernetics, 33(5/6), 877-905.

Zwick, M. (2011a). Reconstructability Analysis of Epistasis. Annals of Human Genetics, 75(1), 157-171. http://doi.org/10.1111/j.1469-1809.2010.00628.x

Zwick, M. (2011b). Reconstructability analysis of epistasis. Annals of Human Genetics, 75(1), 157-71. http://doi.org/10.1111/j.1469-1809.2010.00628.x

Zwick, M. (2011c). Reconstructability Analysis of Epistasis: Reconstructability Analysis of Epistasis. Annals of Human Genetics, 75(1), 157-171. http://doi.org/10.1111/j.1469-1809.2010.00628.x

Zwick, M., \& Johnson, M. S. (2004). State-based reconstructability analysis. Kybernetes, 33(5/6), 1041-1052. http://doi.org/10.1108/03684920410534092 


\section{Appendix: Supplementary Tables}

Table 103. Full List of IVs in Hip and Knee Data Sets with Variable IDs.

\begin{tabular}{|c|c|c|c|}
\hline \# & ICD-9 Code & ICD-9 Description & Variable ID \\
\hline 1 & RISK 135 & Sarcoidosis & Rs \\
\hline 2 & RISK 174.9 & Malignant neoplasm of breast (female), unspecified & $\mathrm{Rb}$ \\
\hline 3 & RISK 185 & Malignant neoplasm of prostate & Rnp \\
\hline 4 & RISK 202.8 & Other malignant lymphomas & Rly \\
\hline 5 & RISK 204.1 & Lymphoid leukemia chronic & Rle \\
\hline 6 & RISK 238.75 & Myelodysplastic syndrome, unspecified & $\mathrm{Rmp}$ \\
\hline 7 & RISK 242 & Thyrotoxicosis with or without goiter & Rth \\
\hline 8 & RISK 244 & Acquired hypothyroidism & $\mathrm{Rh}$ \\
\hline 9 & RISK 244.9 & Unspecified acquired hypothyroidism & Rhy \\
\hline 10 & RISK 246.9 & Unspecified disorder of thyroid & Rtd \\
\hline 11 & RISK 250 & Diabetes mellitus & Rdi \\
\hline 12 & RISK 250.4 & Diabetes with renal manifestations & $\mathrm{Rdr}$ \\
\hline 13 & RISK 250.5 & Diabetes with ophthalmic manifestations & Rdo \\
\hline 14 & RISK 250.6 & Diabetes with neurological manifestations & Rdn \\
\hline 15 & RISK 253.6 & Other disorders of neurohypophysis & Rne \\
\hline 16 & RISK 255.41 & Glucocorticoid deficiency & $\operatorname{Rgd}$ \\
\hline 17 & RISK 257.2 & Other testicular hypofunction & Rte \\
\hline 18 & RISK 266.2 & Other B-complex deficiencies & $\mathrm{Rbc}$ \\
\hline 19 & RISK 268.2 & Osteomalacia, unspecified & Rou \\
\hline 20 & RISK 268.9 & Unspecified vitamin D deficiency & Rvd \\
\hline 21 & RISK 272 & Disorders of lipoid metabolism & Rli \\
\hline 22 & RISK 272.1 & Pure hyperglyceridemia & Rhg \\
\hline 23 & RISK 272.2 & Mixed hyperlipidemia & $\mathrm{Rmh}$ \\
\hline 24 & RISK 272.4 & Other and unspecified hyperlipidemia & Ruh \\
\hline 25 & RISK 274.9 & Gout, unspecified & $\operatorname{Rg}$ \\
\hline 26 & RISK 275.03 & Disorders of phosphorus metabolism & Rpm \\
\hline 27 & RISK 275.49 & Other disorders of calcium metabolism & Red \\
\hline 28 & RISK 276.1 & Hyposmolality and/or hyponatremia & Rhh \\
\hline 29 & RISK 276.8 & Hypopotassemia & Rhp \\
\hline 30 & RISK 277.7 & Dysmetabolic syndrome $\mathrm{X}$ & Rdy \\
\hline 31 & RISK 278 & Overweight, obesity and other hyperalimentation & Roo \\
\hline 32 & RISK 278.01 & Morbid obesity & Rmo \\
\hline 33 & RISK 278.02 & Overweight & Rov \\
\hline 34 & RISK 280 & Iron deficiency anemias & Ria \\
\hline 35 & RISK 280.9 & Iron deficiency anemia, unspecified & Rid \\
\hline 36 & RISK 281.9 & Unspecified deficiency anemia & Ram \\
\hline 37 & RISK 285.1 & Acute posthemorrhagic anemia & Rph \\
\hline 38 & RISK 285.21 & Anemia in chronic kidney disease & Rck \\
\hline 39 & RISK 285.29 & Anemia of other chronic disease & Ran \\
\hline 40 & RISK 285.9 & Anemia, unspecified & Rau \\
\hline 41 & RISK 287.5 & Thrombocytopenia, unspecified & Rtc \\
\hline 42 & RISK 289.81 & Primary hypercoagulable state & Rhs \\
\hline 43 & RISK 294.8 & $\begin{array}{l}\text { Other persistent mental disorders due to conditions classified } \\
\text { elsewhere }\end{array}$ & Rmd \\
\hline 44 & RISK 295.9 & Unspecified schizophrenia & Rsc \\
\hline 45 & RISK 296.8 & Other and unspecified bipolar disorders & Rbp \\
\hline 46 & RISK 300 & Anxiety, dissociative and somatoform disorders & Rad \\
\hline
\end{tabular}




\begin{tabular}{|c|c|c|c|}
\hline \# & ICD-9 Code & ICD-9 Description & Variable ID \\
\hline 47 & RISK 300.01 & Panic disorder with agoraphobia & Rpd \\
\hline 48 & RISK 300.02 & Generalized anxiety disorder & Rga \\
\hline 49 & RISK 300.4 & Dysthymic disorder & Rdd \\
\hline 50 & RISK 303.9 & Other and unspecified alcohol dependence & Rup \\
\hline 51 & RISK 303.91 & Other and unspecified alcohol dependence, continuous & Rah \\
\hline 52 & RISK 303.93 & Other and unspecified alcohol dependence, in remission & Rrn \\
\hline 53 & RISK 304 & Drug dependence & Rdg \\
\hline 54 & RISK 304.01 & Opioid type dependence continuous use & Rop \\
\hline 55 & RISK 305 & Nondependent abuse of drugs & Rab \\
\hline 56 & RISK 305.03 & Alcohol abuse, in remission & Rra \\
\hline 57 & RISK 305.1 & Tobacco use disorder & Rtu \\
\hline 58 & RISK 309.81 & Posttraumatic stress disorder & Rps \\
\hline 59 & RISK 311 & Depressive disorder, not elsewhere classified & Rde \\
\hline 60 & RISK 314 & Hyperkinetic syndrome of childhood & Rhk \\
\hline 61 & RISK 314.01 & Attention deficit disorder with hyperactivity & Rat \\
\hline 62 & RISK 327.23 & Obstructive sleep apnea & Ros \\
\hline 63 & RISK 331 & Other cerebral degenerations & Rce \\
\hline 64 & RISK 332 & Parkinson's disease & Rpa \\
\hline 65 & RISK 333.94 & Restless legs syndrome & Rrl \\
\hline 66 & RISK 338.29 & Other chronic pain & Rcp \\
\hline 67 & RISK 338.4 & Chronic pain syndrome & Rpc \\
\hline 68 & RISK 340 & Multiple sclerosis & Rms \\
\hline 69 & RISK 345.9 & Epilepsy unspecified & Rep \\
\hline 70 & RISK 346.9 & Migraine unspecified & Rmu \\
\hline 71 & RISK 355.9 & Mononeuritis of unspecified site & Rmn \\
\hline 72 & RISK 356.9 & Unspecified hereditary and idiopathic peripheral neuropathy & Rpn \\
\hline 73 & RISK 357.2 & Polyneuropathy in diabetes & Rpo \\
\hline 74 & RISK 357.6 & Polyneuropathy due to drugs & Rpp \\
\hline 75 & RISK 362.01 & Background diabetic retinopathy & Rba \\
\hline 76 & RISK 362.5 & Degeneration of macula and posterior pole of retina & $\mathrm{Rdm}$ \\
\hline 77 & RISK 365.9 & Unspecified glaucoma & Rug \\
\hline 78 & RISK 366.9 & Unspecified cataract & Rcr \\
\hline 79 & RISK 369.4 & Legal blindness, as defined in USA & Rlb \\
\hline 80 & RISK 369.6 & Profound vision impairment one eye & Rip \\
\hline 81 & RISK 369.8 & Unqualified visual loss, one eye & Rvl \\
\hline 82 & RISK 386 & $\begin{array}{l}\text { Vertiginous syndromes and other disorders of vestibular } \\
\text { system }\end{array}$ & Rve \\
\hline 83 & RISK 388.3 & Tinnitus & Rti \\
\hline 84 & RISK 389.9 & Unspecified hearing loss & Rhl \\
\hline 85 & RISK 397 & Diseases of other endocardial structures & Res \\
\hline 86 & RISK 401.1 & Benign essential hypertension & Rbe \\
\hline 87 & RISK 401.9 & Unspecified essential hypertension & Rhe \\
\hline 88 & RISK 403.9 & Unspecified hypertensive renal disease & Rrd \\
\hline 89 & RISK 413.9 & Other and unspecified angina pectoris & Rap \\
\hline 90 & RISK 414 & Other forms of chronic ischemic heart disease & Rci \\
\hline 91 & RISK 414.01 & Coronary atherosclerosis of native coronary artery & Rca \\
\hline 92 & RISK 414.8 & Other specified forms of chronic ischemic heart disease & Rsf \\
\hline 93 & RISK 416.8 & Other chronic pulmonary heart diseases & Rhd \\
\hline 94 & RISK 424 & Other diseases of endocardium & Rec \\
\hline 95 & RISK 424.1 & Aortic valve disorders & Rav \\
\hline 96 & RISK 425.4 & Other primary cardiomyopathies & $\mathrm{Rcm}$ \\
\hline
\end{tabular}




\begin{tabular}{|c|c|c|c|}
\hline \# & ICD-9 Code & ICD-9 Description & Variable ID \\
\hline 97 & RISK 426.11 & First degree atrioventricular block & Rfd \\
\hline 98 & RISK 426.3 & Other left bundle branch block & Rol \\
\hline 99 & RISK 426.4 & Right bundle branch block & Rrb \\
\hline 100 & RISK 427 & Cardiac dysrhythmias & Rys \\
\hline 101 & RISK 427.31 & Atrial fibrillation & Raf \\
\hline 102 & RISK 427.32 & Atrial flutter & Rfl \\
\hline 103 & RISK 427.69 & Other premature beats & $\mathrm{Rpb}$ \\
\hline 104 & RISK 427.89 & Other specified cardiac dysrhythmias & Rod \\
\hline 105 & RISK 427.9 & Cardiac dysrhythmia, unspecified & Ruc \\
\hline 106 & RISK 428 & Heart failure & Rhf \\
\hline 107 & RISK 428.3 & Diastolic heart failure & Rdh \\
\hline 108 & RISK 428.32 & Chronic diastolic heart failure & Rdf \\
\hline 109 & RISK 429.9 & Heart disease, unspecified & Rht \\
\hline 110 & RISK 433.1 & Occlusion and stenosis of carotid artery & Roc \\
\hline 111 & RISK 441.4 & Abdominal aneurysm without mention of rupture & Raa \\
\hline 112 & RISK 443 & Other peripheral vascular disease & Rpe \\
\hline 113 & RISK 443.9 & Peripheral vascular disease, unspecified & Rpv \\
\hline 114 & RISK 457.1 & Other lymphedema & Roe \\
\hline 115 & RISK 458.9 & Hypotension, unspecified & Rho \\
\hline 116 & RISK 459.81 & Venous (peripheral) insufficiency, unspecified & Rvi \\
\hline 117 & RISK 477 & Allergic rhinitis & Rar \\
\hline 118 & RISK 477.9 & Allergic rhinitis, cause unspecified & Ral \\
\hline 119 & RISK 491.2 & Obstructive chronic bronchitis & Rcb \\
\hline 120 & RISK 491.9 & Unspecified chronic bronchitis & Rbh \\
\hline 121 & RISK 492.8 & Other emphysema & Rem \\
\hline 122 & RISK 493 & Asthma & Ras \\
\hline 123 & RISK 493.2 & Chronic obstructive asthma & Rco \\
\hline 124 & RISK 493.9 & Asthma unspecified & Rua \\
\hline 125 & RISK 496 & Chronic airway obstruction, not elsewhere classified & Rao \\
\hline 126 & RISK 515 & Postinflammatory pulmonary fibrosis & Rpf \\
\hline 127 & RISK 518.89 & Other diseases of lung, not elsewhere classified & Rld \\
\hline 128 & RISK 530.1 & Esophagitis & Reg \\
\hline 129 & RISK 530.11 & Reflux esophagitis & Rre \\
\hline 130 & RISK 530.81 & Esophageal reflux & Rer \\
\hline 131 & RISK 530.85 & Barrett's esophagus & Rbr \\
\hline 132 & RISK 553.3 & $\begin{array}{l}\text { Diaphragmatic hernia without mention of obstruction or } \\
\text { gangrene }\end{array}$ & Rhw \\
\hline 133 & RISK 555.9 & Regional enteritis of unspecified site & $\operatorname{Rrg}$ \\
\hline 134 & RISK 556.9 & Ulcerative colitis, unspecified & $\mathrm{Rcl}$ \\
\hline 135 & RISK 558.9 & $\begin{array}{l}\text { Other and unspecified noninfectious gastroenteritis and } \\
\text { colitis }\end{array}$ & Rng \\
\hline 136 & RISK 562.1 & Diverticula of colon & $\operatorname{Rdv}$ \\
\hline 137 & RISK 564 & Functional digestive disorders not elsewhere classified & $\mathrm{Rfu}$ \\
\hline 138 & RISK 564.09 & Other constipation & Res \\
\hline 139 & RISK 564.1 & Irritable bowel syndrome & Rir \\
\hline 140 & RISK 571.8 & Other chronic nonalcoholic liver disease & Rnl \\
\hline 141 & RISK 583.81 & $\begin{array}{l}\text { Nephritis and nephropathy, not specified as acute or chronic, } \\
\text { in diseases classified elsewhere }\end{array}$ & Rnn \\
\hline 142 & RISK 585.2 & Chronic kidney disease, Stage II (mild) & Rki \\
\hline 143 & RISK 585.3 & Chronic kidney disease, Stage III (moderate) & Rkd \\
\hline 144 & RISK 585.4 & Chronic kidney disease, Stage IV (severe) & Rks \\
\hline
\end{tabular}




\begin{tabular}{|c|c|c|c|}
\hline \# & ICD-9 Code & ICD-9 Description & Variable ID \\
\hline 145 & RISK 585.9 & Chronic kidney disease, unspecified & Rku \\
\hline 146 & RISK 593.9 & Unspecified disorder of kidney and ureter & Rud \\
\hline 147 & RISK 596 & Other disorders of bladder & $\mathrm{Rdb}$ \\
\hline 148 & RISK 599 & Other disorders of urethra and urinary tract & Ruu \\
\hline 149 & RISK 600 & Hyperplasia of prostate & Rpl \\
\hline 150 & RISK 600.01 & $\begin{array}{l}\text { Hypertrophy (benign) of prostate and urinary obstruction and } \\
\text { lower urinary tract symptoms }\end{array}$ & Ruo \\
\hline 151 & RISK 600.9 & Hyperplasia of prostate unspecified & $\mathrm{Rpu}$ \\
\hline 152 & RISK 601.9 & Prostatis, unspecified & Rpt \\
\hline 153 & RISK 607.84 & Impotence of organic origin & Rim \\
\hline 154 & RISK 625.6 & Stress incontinence, female & Rsi \\
\hline 155 & RISK 695.3 & Rosacea & Rro \\
\hline 156 & RISK 696 & Psoriasis and similar disorders & Rsd \\
\hline 157 & RISK 696.1 & Other psoriasis & Rsr \\
\hline 158 & RISK 70.54 & Repair of cystocele with graft or prosthesis & $\operatorname{Rgp}$ \\
\hline 159 & RISK 70.7 & Other Repair of Vagina & Rrv \\
\hline 160 & RISK 710 & Diffuse diseases of connective tissue & Rct \\
\hline 161 & RISK 710.2 & Sicca syndrome & Rss \\
\hline 162 & RISK 712.36 & Chondrocalcinosis, unspecified lower leg & Rcu \\
\hline 163 & RISK 714 & $\begin{array}{l}\text { Rheumatoid arthritis and other inflammatory } \\
\text { polyarthropathies }\end{array}$ & Rrh \\
\hline 164 & RISK 715.15 & Osteoarthrosis, localized, primary, pelvic region and thigh & Rlp \\
\hline 165 & RISK 715.25 & Osteoarthrosis, localized, secondary, pelvic region and thigh & Roh \\
\hline 166 & RISK 715.35 & $\begin{array}{l}\text { Osteoarthrosis, localized, not specified whether primary or } \\
\text { secondary, pelvic region and thigh }\end{array}$ & Roa \\
\hline 167 & RISK 715.36 & $\begin{array}{l}\text { Osteoarthrosis, localized, not specified whether primary or } \\
\text { secondary, lower leg }\end{array}$ & Rll \\
\hline 168 & RISK 715.9 & Osteoarthrosis unspecified whether generalized or localized & Rgo \\
\hline 169 & RISK 715.95 & $\begin{array}{l}\text { Osteoarthrosis, unspecified whether generalized or localized, } \\
\text { pelvic region and thigh }\end{array}$ & Rgt \\
\hline 170 & RISK 715.96 & $\begin{array}{l}\text { Osteoarthrosis, unspecified whether generalized or localized, } \\
\text { lower leg }\end{array}$ & Rog \\
\hline 171 & RISK 716.16 & Traumatic arthropathy, lower leg & Rta \\
\hline 172 & RISK 716.95 & Arthropathy, unspecified, pelvic region and thigh & Ray \\
\hline 173 & RISK 716.96 & Arthropathy, unspecified, lower leg & Rul \\
\hline 174 & RISK 717.6 & Loose body in knee & Rlo \\
\hline 175 & RISK 717.7 & Chondromalacia of patella & Rch \\
\hline 176 & RISK 718.45 & Contracture of joint, pelvic region and thigh & Rjc \\
\hline 177 & RISK 718.46 & Contracture of joint, lower leg & Rcj \\
\hline 178 & RISK 719.06 & Effusion of joint, lower leg & Rej \\
\hline 179 & RISK 719.46 & Pain in joint, lower leg & Rjp \\
\hline 180 & RISK 721 & Spondylosis and allied disorders & Rso \\
\hline 181 & RISK 721.3 & Lumbosacral spondylosis without myelopathy & Rls \\
\hline 182 & RISK 721.9 & Spondylosis of unspecified site & Rsu \\
\hline 183 & RISK 722.4 & Degeneration of cervical intervertebral disc & Ric \\
\hline 184 & RISK 722.52 & Degeneration of lumbar or lumbosacral intervertebral disc & Rdl \\
\hline 185 & RISK 724 & Other and unspecified disorders of back & Rdu \\
\hline 186 & RISK 724.02 & $\begin{array}{l}\text { Spinal stenosis, lumbar region, without neurogenic } \\
\text { claudication }\end{array}$ & Rsp \\
\hline 187 & RISK 724.2 & Lumbago & Rlu \\
\hline 188 & RISK 724.3 & Sciatica & Rsa \\
\hline
\end{tabular}




\begin{tabular}{|c|c|c|c|}
\hline \# & ICD-9 Code & ICD-9 Description & Variable ID \\
\hline 189 & RISK 724.5 & Backache, unspecified & Rbu \\
\hline 190 & RISK 725 & Polymyalgia rheumatica & Rpy \\
\hline 191 & RISK 726.5 & Enthesopathy of hip region & Ren \\
\hline 192 & RISK 727 & Other disorders of synovium tendon and bursa & Rst \\
\hline 193 & RISK 727.09 & Other synovitis and tenosynovitis & Rsy \\
\hline 194 & RISK 728.87 & Muscle weakness (generalized) & Rmw \\
\hline 195 & RISK 729.1 & Myalgia and myositis, unspecified & Rma \\
\hline 196 & RISK 731 & $\begin{array}{l}\text { Oseitis deformans and osteopathies associated with other } \\
\text { disorders classified elsewhere }\end{array}$ & Rot \\
\hline 197 & RISK 732.1 & Juvenile osteochondrosis of hip and pelvis & Rjo \\
\hline 198 & RISK 733 & Other disorders of bone and cartilage & Rbn \\
\hline 199 & RISK 733.01 & Senile osteoporosis & Rse \\
\hline 200 & RISK 733.2 & Cyst of bone & Rcy \\
\hline 201 & RISK 733.29 & Other bone cyst & Rob \\
\hline 202 & RISK 733.4 & Aseptic necrosis of bone & Rnb \\
\hline 203 & RISK 733.42 & Aseptic necrosis of head and neck of femur & Rnr \\
\hline 204 & RISK 733.82 & Nonunion of fracture & Rfr \\
\hline 205 & RISK 733.9 & Other and unspecified disorders of bone and cartilage & $\mathrm{Rdc}$ \\
\hline 206 & RISK 733.99 & Other disorders of bone and cartilage & $\operatorname{Rcg}$ \\
\hline 207 & RISK 736.6 & Other acquired deformities of knee & Raq \\
\hline 208 & RISK 736.79 & Other acquired deformities of ankle and foot & Rak \\
\hline 209 & RISK 736.81 & Unequal leg length (acquired) & Rla \\
\hline 210 & RISK 736.89 & Other acquired deformity of other parts of limb & Rdp \\
\hline 211 & RISK 737.3 & Kyphoscoliosis and scoliosis & Rky \\
\hline 212 & RISK 738.4 & Acquired spondylolisthesis & Rsl \\
\hline 213 & RISK 755.63 & Other congenital deformity of hip (joint) & Rhj \\
\hline 214 & RISK 780.4 & Dizziness and giddiness & $\mathrm{Rdz}$ \\
\hline 215 & RISK 780.52 & Insomnia, unspecified & Riu \\
\hline 216 & RISK 780.57 & Unspecified sleep apnea & Rae \\
\hline 217 & RISK 780.93 & Memory loss & $\mathrm{Rml}$ \\
\hline 218 & RISK 782.3 & Edema & Red \\
\hline 219 & RISK 784 & Symptoms involving head and neck & Rhn \\
\hline 220 & RISK 785 & Symtoms involving cardiovascular system & Rev \\
\hline 221 & RISK 785.2 & Undiagnosed cardiac murmurs & Rum \\
\hline 222 & RISK 788.2 & Retention of urine & Rur \\
\hline 223 & RISK 788.3 & Urinary incontinence & Rin \\
\hline 224 & RISK 788.31 & Urge incontinence & Rit \\
\hline 225 & RISK 788.41 & Urinary frequency & Ruf \\
\hline 226 & RISK 790.29 & Other abnormal glucose & Rgl \\
\hline 227 & RISK 790.92 & Abnormal coagulation profile & $\mathrm{Rpr}$ \\
\hline 228 & RISK E932.0 & $\begin{array}{l}\text { Adrenal cortical steroids causing adverse effects in } \\
\text { therapeutic use }\end{array}$ & Ref \\
\hline 229 & RISK E933.1 & $\begin{array}{l}\text { Antineoplastic and immunosuppressive drugs causing } \\
\text { adverse effects in therapeutic use }\end{array}$ & Rai \\
\hline 230 & RISK V08 & $\begin{array}{l}\text { Asymptomatic human immunodeficiency virus (HIV) } \\
\text { infection status }\end{array}$ & Rhi \\
\hline 231 & RISK V85.4 & Body mass index 40 and over, adult & Rmi \\
\hline
\end{tabular}


Table 104. Literature-based IVs Retained for Hip and Knee Data Sets after Variable Reduction.

\begin{tabular}{|c|c|c|c|c|c|c|}
\hline \# & ICD-9 Code & ICD-9 Description & Var. ID & \begin{tabular}{|c|} 
Literature- \\
based IVs \\
(Bozic/Jain \\
Study) \\
\end{tabular} & $\begin{array}{l}\text { IV in } \\
\text { Knee }\end{array}$ & $\begin{array}{l}\text { IV in } \\
\text { Hip }\end{array}$ \\
\hline 1 & RISK 250 & Diabetes mellitus & Rdi & \begin{tabular}{|c|} 
yes \\
\end{tabular} & yes & yes \\
\hline 2 & RISK 250.4 & Diabetes with renal manifestations & $\mathrm{Rdr}$ & yes & yes & no \\
\hline 3 & RISK 250.5 & Diabetes with ophthalmic manifestations & Rdo & yes & yes & no \\
\hline 4 & RISK 250.6 & Diabetes with neurological manifestations & $\operatorname{Rdn}$ & yes & yes & yes \\
\hline 5 & RISK 278 & $\begin{array}{l}\text { Overweight, obesity and other } \\
\text { hyperalimentation }\end{array}$ & Roo & yes & yes & yes \\
\hline 6 & RISK 278.01 & Morbid obesity & Rmo & yes & yes & yes \\
\hline 7 & RISK 278.02 & Overweight & Rov & yes & yes & yes \\
\hline 8 & RISK 401.1 & Benign essential hypertension & Rbe & yes & yes & yes \\
\hline 9 & RISK 401.9 & Unspecified essential hypertension & Rhe & yes & yes & yes \\
\hline 10 & RISK 414 & Other forms of chronic ischemic heart disease & Rci & yes & yes & yes \\
\hline 11 & RISK 414.01 & $\begin{array}{l}\text { Coronary atherosclerosis of native coronary } \\
\text { artery }\end{array}$ & Rca & yes & yes & yes \\
\hline 12 & RISK 428 & Heart failure & Rhf & yes & yes & yes \\
\hline 13 & RISK 428.3 & Diastolic heart failure & $\mathrm{Rdh}$ & yes & yes & yes \\
\hline 14 & RISK 428.32 & Chronic diastolic heart failure & Rdf & yes & yes & no \\
\hline 15 & RISK 443 & Other peripheral vascular disease & Rpe & yes & yes & yes \\
\hline 16 & RISK 443.9 & Peripheral vascular disease, unspecified & Rpv & yes & yes & yes \\
\hline 17 & RISK 491.2 & Obstructive chronic bronchitis & Rcb & yes & yes & no \\
\hline 18 & RISK 491.9 & Unspecified chronic bronchitis & Rbh & yes & yes & no \\
\hline 19 & RISK 492.8 & Other emphysema & Rem & yes & yes & yes \\
\hline 20 & RISK 493 & Asthma & Ras & yes & yes & no \\
\hline 21 & RISK 493.2 & Chronic obstructive asthma & Rco & yes & yes & yes \\
\hline 22 & RISK 493.9 & Asthma unspecified & Rua & yes & yes & yes \\
\hline 23 & RISK 496 & $\begin{array}{l}\text { Chronic airway obstruction, not elsewhere } \\
\text { classified }\end{array}$ & Rao & yes & yes & yes \\
\hline
\end{tabular}


Table 105. 62 Single Predicting IVs for DV SNF (Hip data).

\begin{tabular}{|c|c|c|c|c|c|c|c|c|c|}
\hline MODEL & dDF & dLR & Alpha & $\% \mathrm{dH}(\mathrm{DV})$ & dAIC & dBIC & Inc.Alpha & $p<0.05$ & Keep Lit \\
\hline Rhy & 1 & 38.48 & 0.00 & 1.47 & 36.48 & 30.41 & 0.00 & yes & \\
\hline Rhe & 1 & 33.53 & 0.00 & 1.28 & 31.53 & 25.46 & 0.00 & yes & yes \\
\hline Rav & 1 & 24.07 & 0.00 & 0.92 & 22.07 & 15.99 & 0.00 & yes & \\
\hline Rrd & 1 & 23.96 & 0.00 & 0.91 & 21.96 & 15.89 & 0.00 & yes & \\
\hline Rao & 1 & 23.68 & 0.00 & 0.90 & 21.68 & 15.61 & 0.00 & yes & yes \\
\hline $\mathrm{Rhf}$ & 1 & 20.94 & 0.00 & 0.80 & 18.94 & 12.87 & 0.00 & yes & yes \\
\hline Rnr & 1 & 19.36 & 0.00 & 0.74 & 17.36 & 11.29 & 0.00 & yes & \\
\hline Rbn & 1 & 18.32 & 0.00 & 0.70 & 16.32 & 10.25 & 0.00 & yes & \\
\hline Rhh & 1 & 16.05 & 0.00 & 0.61 & 14.05 & 7.98 & 0.00 & yes & \\
\hline Rkd & 1 & 15.87 & 0.00 & 0.60 & 13.87 & 7.80 & 0.00 & yes & \\
\hline Rdi & 1 & 15.85 & 0.00 & 0.60 & 13.85 & 7.77 & 0.00 & yes & yes \\
\hline Rin & 1 & 14.43 & 0.00 & 0.55 & 12.43 & 6.36 & 0.00 & yes & \\
\hline Rse & 1 & 14.15 & 0.00 & 0.54 & 12.15 & 6.08 & 0.00 & yes & \\
\hline Raf & 1 & 13.66 & 0.00 & 0.52 & 11.66 & 5.59 & 0.00 & yes & \\
\hline Rci & 1 & 13.22 & 0.00 & 0.50 & 11.22 & 5.15 & 0.00 & yes & yes \\
\hline Rhd & 1 & 12.89 & 0.00 & 0.49 & 10.89 & 4.82 & 0.00 & yes & \\
\hline Rau & 1 & 12.38 & 0.00 & 0.47 & 10.38 & 4.31 & 0.00 & yes & \\
\hline Rku & 1 & 11.28 & 0.00 & 0.43 & 9.28 & 3.21 & 0.00 & yes & \\
\hline Rug & 1 & 11.28 & 0.00 & 0.43 & 9.28 & 3.21 & 0.00 & yes & \\
\hline Rdh & 1 & 10.91 & 0.00 & 0.42 & 8.91 & 2.84 & 0.00 & yes & yes \\
\hline $\mathrm{Rml}$ & 1 & 10.91 & 0.00 & 0.42 & 8.91 & 2.84 & 0.00 & yes & \\
\hline Rop & 1 & 9.80 & 0.00 & 0.37 & 7.80 & 1.73 & 0.00 & yes & \\
\hline $\mathrm{Rdm}$ & 1 & 8.66 & 0.00 & 0.33 & 6.66 & 0.59 & 0.00 & yes & \\
\hline Rld & 1 & 8.58 & 0.00 & 0.33 & 6.58 & 0.51 & 0.00 & yes & \\
\hline Rsi & 1 & 8.38 & 0.00 & 0.32 & 6.38 & 0.30 & 0.00 & yes & \\
\hline Rca & 1 & 8.37 & 0.00 & 0.32 & 6.37 & 0.29 & 0.00 & yes & yes \\
\hline Rhl & 1 & 7.46 & 0.01 & 0.28 & 5.46 & -0.62 & 0.01 & yes & \\
\hline Rod & 1 & 7.38 & 0.01 & 0.28 & 5.38 & -0.69 & 0.01 & yes & \\
\hline Rph & 1 & 6.97 & 0.01 & 0.27 & 4.97 & -1.11 & 0.01 & yes & \\
\hline Rvi & 1 & 6.75 & 0.01 & 0.26 & 4.75 & -1.32 & 0.01 & yes & \\
\hline $\operatorname{Rec}$ & 1 & 6.75 & 0.01 & 0.26 & 4.75 & -1.32 & 0.01 & yes & \\
\hline Rhj & 1 & 6.34 & 0.01 & 0.24 & 4.34 & -1.73 & 0.01 & yes & \\
\hline Rmd & 1 & 6.19 & 0.01 & 0.24 & 4.19 & -1.88 & 0.01 & yes & \\
\hline $\mathrm{Rpc}$ & 1 & 6.19 & 0.01 & 0.24 & 4.19 & -1.88 & 0.01 & yes & \\
\hline Ruu & 1 & 6.19 & 0.01 & 0.24 & 4.19 & -1.88 & 0.01 & yes & \\
\hline $\operatorname{Rog}$ & 1 & 5.80 & 0.02 & 0.22 & 3.80 & -2.27 & 0.02 & yes & \\
\hline Rcg & 1 & 5.58 & 0.02 & 0.21 & 3.58 & -2.49 & 0.02 & yes & \\
\hline Rip & 1 & 5.58 & 0.02 & 0.21 & 3.58 & -2.49 & 0.02 & yes & \\
\hline $\operatorname{Rad}$ & 1 & 5.08 & 0.02 & 0.19 & 3.08 & -2.99 & 0.02 & yes & \\
\hline $\mathrm{Rh}$ & 1 & 4.94 & 0.03 & 0.19 & 2.94 & -3.13 & 0.03 & yes & \\
\hline $\mathrm{Rdl}$ & 1 & 4.77 & 0.03 & 0.18 & 2.77 & -3.30 & 0.03 & yes & \\
\hline Rsp & 1 & 4.64 & 0.03 & 0.18 & 2.64 & -3.43 & 0.03 & yes & \\
\hline Rrn & 1 & 4.32 & 0.04 & 0.16 & 2.32 & -3.75 & 0.04 & yes & \\
\hline Ram & 1 & 4.30 & 0.04 & 0.16 & 2.30 & -3.77 & 0.04 & yes & \\
\hline Rap & 1 & 4.30 & 0.04 & 0.16 & 2.30 & -3.77 & 0.04 & yes & \\
\hline Ray & 1 & 4.30 & 0.04 & 0.16 & 2.30 & -3.77 & 0.04 & yes & \\
\hline Ria & 1 & 4.30 & 0.04 & 0.16 & 2.30 & -3.77 & 0.04 & yes & \\
\hline Rem & 1 & 4.18 & 0.04 & 0.16 & 2.18 & -3.89 & 0.04 & yes & yes \\
\hline Rpn & 1 & 4.16 & 0.04 & 0.16 & 2.16 & -3.91 & 0.04 & yes & \\
\hline
\end{tabular}




\begin{tabular}{|l|c|c|c|c|c|c|c|c|c|}
\hline MODEL & dDF & dLR & Alpha & \%dH(DV) & dAIC & dBIC & Inc.Alpha & p $<0.05$ & Keep Lit \\
\hline Rpo & 1 & 4.02 & 0.05 & 0.15 & 2.02 & -4.05 & 0.05 & yes & \\
\hline Roc & 1 & 3.98 & 0.05 & 0.15 & 1.98 & -4.09 & 0.05 & yes & \\
\hline Rra & 1 & 3.98 & 0.05 & 0.15 & 1.98 & -4.09 & 0.05 & yes & \\
\hline Rde & 1 & 3.76 & 0.05 & 0.14 & 1.76 & -4.32 & 0.05 & yes & \\
\hline Rbe & 1 & 3.08 & 0.08 & 0.12 & 1.08 & -4.99 & 0.08 & & yes \\
\hline Rpv & 1 & 2.85 & 0.09 & 0.11 & 0.85 & -5.22 & 0.09 & & yes \\
\hline Rdn & 1 & 2.78 & 0.10 & 0.11 & 0.78 & -5.29 & 0.10 & & yes \\
\hline Roo & 1 & 1.28 & 0.26 & 0.05 & -0.72 & -6.79 & 0.26 & & yes \\
\hline Rpe & 1 & 1.26 & 0.26 & 0.05 & -0.74 & -6.81 & 0.26 & & yes \\
\hline Rco & 1 & 1.05 & 0.30 & 0.04 & -0.95 & -7.02 & 0.30 & & yes \\
\hline Rov & 1 & 0.86 & 0.35 & 0.03 & -1.14 & -7.21 & 0.35 & & yes \\
\hline Rmo & 1 & 0.68 & 0.41 & 0.03 & -1.32 & -7.39 & 0.41 & & yes \\
\hline Rua & 1 & 0.32 & 0.57 & 0.01 & -1.68 & -7.76 & 0.57 & & yes \\
\hline
\end{tabular}

\section{Component Fit Tables for Knee SNF (All IVs)}

Table 106. Component Fit Table for IV Ageb in (Knee) All IVs

Best Model L SNF : Fc SNF : Ageb SNF : Svb SNF : Nrb SNF : Rmd SNF : Rug SNF : Rhf SNF. Blue rows indicate ratio $<0.90$ and orange rows indicate ratio $>1.10$.

\begin{tabular}{|l|l|l|l|l|l|l|}
\hline & IV & Data & \multicolumn{2}{l|}{ obs. p(DV|IV) } & & \\
\hline$\#$ & Ageb & freq & SNF=1 & SNF=2 & Ratio & p(margin) \\
\hline 1 & 1 & 1490 & 94.50 & 5.50 & 0.31 & 0.00 \\
\hline 2 & 2 & 1411 & 86.11 & 13.89 & 0.79 & 0.00 \\
\hline 3 & 3 & 1435 & 66.34 & 33.66 & 1.92 & 0.00 \\
\hline & & 4336 & 82.45 & 17.55 & 1.00 & \\
\hline
\end{tabular}

Table 107. Component Fit Table for IV Svb in (Knee) All IVS

Best Model L SNF : Fc SNF : Ageb SNF : Svb SNF : Nrb SNF : Rmd SNF : Rug SNF : Rhf SNF.

Blue rows indicate ratio $<0.90$ and orange rows indicate ratio $>1.10$.

(Ratios between 0.91 and 1.09 are close to the margins and are excluded.)

\begin{tabular}{|l|l|l|l|l|l|l|}
\hline & IV & Data & \multicolumn{2}{|l|}{ obs. $p$ (DV|IV) } & & \\
\hline$\#$ & Svb & freq & SNF=1 & SNF=2 & Ratio & p(margin) \\
\hline 1 & 2 & 1518 & 78.33 & 21.67 & 1.23 & 0.00 \\
\hline 2 & 3 & 1374 & 86.25 & 13.76 & 0.78 & 0.00 \\
\hline & & 4336 & 82.45 & 17.55 & 1.00 & \\
\hline
\end{tabular}


Table 108. Component Fit Table for IV Nrb in (Knee) All IVs

Best Model L SNF : Fc SNF : Ageb SNF : Svb SNF : Nrb SNF : Rmd SNF : Rug SNF : Rhf SNF.

Blue rows indicate ratio $<0.90$ and orange rows indicate ratio $>1.10$.

(Ratios between 0.91 and 1.09 are close to the margins and are excluded.)

\begin{tabular}{|l|l|l|l|l|l|l|}
\hline & IV & Data & \multicolumn{2}{l|}{ obs. p(DV|IV) } & & \\
\hline$\#$ & Nrb & freq & SNF=1 & SNF=2 & Ratio & p(margin) \\
\hline 1 & 1 & 1309 & 89.61 & 10.39 & 0.59 & 0.00 \\
\hline 2 & 3 & 1553 & 75.34 & 24.66 & 1.41 & 0.00 \\
\hline & & 4336 & 82.45 & 17.55 & 1.00 & \\
\hline
\end{tabular}

Table 109. Condensed Component Fit Table for Rmd, Rug, and Rhf for (Knee) All IVs for IV Rmd. Blue rows indicate ratio $<0.90$ and orange rows indicate ratio $>1.10$.

(Ratios between 0.91 and 1.09 are close to the margins and are excluded.)

\begin{tabular}{|c|c|c|c|c|c|c|}
\hline & & Data & obs. p(DV|IV) & & & \\
\hline IV & State & freq & $\mathrm{SNF}=1$ & $\mathrm{SNF}=2$ & Ratio & $\mathrm{p}$ (margin) \\
\hline Rmd & 1 & 19 & 21.05 & 78.95 & 4.50 & 0 \\
\hline Rug & 1 & 86 & 54.65 & 45.35 & 2.58 & 0 \\
\hline Rhf & 1 & 87 & 54.02 & 45.98 & 2.62 & 0 \\
\hline & & 4336 & 82.45 & 17.55 & 1.00 & \\
\hline
\end{tabular}

\section{Component Fit Tables for Knee (Comorbidity IVs Only)}

Table 110. Condensed Component Fit Table for IVs Rmd, Rpa, Rug, Rhe, Rhe, Rhf, Rku, Rbn, and Rin for (Knee) Comorbidity IVs

Best Model Rhy SNF : Rau SNF : Rmd SNF : Rpa SNF : Rug SNF : Rhe SNF :

Rhf SNF : Rku SNF : Rbn SNF : Rin SNF.

Blue rows indicate ratio $<0.90$ and orange rows indicate ratio $>1.10$.

(Ratios between 0.91 and 1.09 are close to the margins and are excluded.)

\begin{tabular}{|c|c|c|c|c|c|c|}
\hline & & Data & obs. p(DV|IV) & & & \\
\hline IV & State & freq & $\mathrm{SNF}=1$ & $\mathrm{SNF}=2$ & ratio & $\mathrm{p}($ margin $)$ \\
\hline Rmd & 1 & 19 & 21.05 & 78.95 & 4.50 & 0.00 \\
\hline Rpa & 1 & 26 & 53.85 & 46.15 & 2.63 & 0.00 \\
\hline Rug & 1 & 86 & 54.65 & 45.35 & 2.58 & 0.00 \\
\hline Rhe & 0 & 1963 & 84.77 & 15.23 & 0.87 & 0.01 \\
\hline Rhe & 1 & 2373 & 80.53 & 19.47 & 1.11 & 0.01 \\
\hline $\mathrm{Rhf}$ & 1 & 87 & 54.02 & 45.98 & 2.62 & 0.00 \\
\hline $\mathrm{Rku}$ & 1 & 87 & 57.47 & 42.53 & 2.42 & 0.00 \\
\hline Rbn & 1 & 149 & 65.77 & 34.23 & 1.95 & 0.00 \\
\hline Rin & 1 & 40 & 50.00 & 50.00 & 2.85 & 0.00 \\
\hline & & 4336 & 82.45 & 17.55 & 1.00 & \\
\hline
\end{tabular}




\section{Component Fit Tables for Hip SNF (All IVs)}

Table 111. Component Fit Table for IV Fc in (Hip) All IVs

Best Model Fe SNF : Ageb SNF : Svb SNF : Nrb SNF.

Blue rows indicate ratio $<0.90$ and orange rows indicate ratio $>1.10$.

(Frequencies $<10$ are excluded and ratios between 0.91 and 1.09 are close to the margins and are excluded.)

\begin{tabular}{|l|l|l|l|l|l|l|}
\hline & IV & Data & obs. p(DV|IV) & & \\
\hline$\#$ & Fc & freq & SNF=1 & SNF=2 & Ratio & p(margin) \\
\hline 1 & 1 & 1598 & 75.78 & 24.22 & 1.70 & 0.00 \\
\hline 2 & 2 & 1501 & 96.67 & 3.33 & 0.23 & 0.00 \\
\hline 3 & 3 & 61 & 70.49 & 29.51 & 2.07 & 0.00 \\
\hline 6 & 6 & 19 & 100.00 & 0.00 & 0.00 & 0.08 \\
\hline & & 3204 & 85.74 & 14.26 & & \\
\hline
\end{tabular}

Table 112. Component Fit Table for IV Ageb in (Hip) All IVs

Best Model Fc SNF : Ageb SNF : Svb SNF : Nrb SNF.

Blue rows indicate ratio $<0.90$ and orange rows indicate ratio $>1.10$.

\begin{tabular}{|c|c|c|c|c|c|c|}
\hline & IV & Data & obs. p(DV|IV & & & \\
\hline \# & Ageb & freq & $\mathrm{SNF}=1$ & $\mathrm{SNF}=2$ & Ratio & $\mathrm{p}($ margin $)$ \\
\hline 1 & 1 & 1089 & 95.23 & 4.78 & 0.33 & 0.00 \\
\hline 2 & 2 & 1027 & 92.11 & 7.89 & 0.55 & 0.00 \\
\hline 3 & 3 & 1088 & 70.22 & 29.78 & 2.09 & 0.00 \\
\hline & & 3204 & 85.74 & 14.26 & 1.00 & \\
\hline
\end{tabular}

Table 113. Component Fit Table for IV Svb in (Hip) All IVs

Best Model Fc SNF : Ageb SNF : Svb SNF : Nrb SNF.

Blue rows indicate ratio $<0.90$ and orange rows indicate ratio $>1.10$.

\begin{tabular}{|c|c|c|c|c|c|c|}
\hline & IV & Data & obs. p(DV|IV & & & \\
\hline$\#$ & Svb & freq & $\mathrm{SNF}=1$ & $\mathrm{SNF}=2$ & Ratio & $\mathrm{p}($ margin $)$ \\
\hline 1 & 1 & 1067 & 82.19 & 17.81 & 1.25 & 0.00 \\
\hline 2 & 2 & 1053 & 78.16 & 21.84 & 1.53 & 0.00 \\
\hline 3 & 3 & 1084 & 96.59 & 3.41 & 0.24 & 0.00 \\
\hline & & 3204 & 85.74 & 14.26 & 1.00 & \\
\hline
\end{tabular}

Table 114. Component Fit Table for IV Nrb in (Hip) All IVs

Best Model Fc SNF : Ageb SNF : Svb SNF : Nrb SNF.

Blue rows indicate ratio $<0.90$ and orange rows indicate ratio $>1.10$.

(Ratios between 0.91 and 1.09 are close to the margins and are excluded.)

\begin{tabular}{|l|l|l|l|l|l|l|}
\hline & IV & Data & obs. p(DV|IV) & & \\
\hline$\#$ & Nrb & freq & SNF=1 & SNF=2 & Ratio & p(margin) \\
\hline 1 & 1 & 1111 & 91.81 & 8.19 & 0.57 & 0.00 \\
\hline 2 & 3 & 1012 & 77.17 & 22.83 & 1.60 & 0.00 \\
\hline & 3204 & 85.74 & 14.26 & 1.00 & \\
\hline
\end{tabular}


Table 115. Fit Table (Hip) for Comorbidity IVs for

Best Model Rhy SNF : Rhh SNF : Rug SNF :Rhe SNF : Rrd SNF :

Rav Rbn SNF : Rao SNF : Rse SNF : Rnr SNF.

Blue rows indicate ratio $<0.90$ and orange rows indicate ratio $>1.10$.

(Ratios between 0.91 and 1.09 are close to the margins and are indicated in gray.)

\begin{tabular}{|c|c|c|c|c|c|c|c|c|c|c|c|c|c|c|c|c|c|}
\hline \multicolumn{11}{|c|}{ IVs } & \multicolumn{3}{|l|}{ Data } & \multicolumn{2}{|c|}{ Model } & \multirow[b]{3}{*}{ Ratio } & \multirow[b]{3}{*}{$\mathrm{p}$ (margin) } \\
\hline & & & & & & & & & & & & \multicolumn{2}{|c|}{ obs. p(DV|IV) } & calc. $\mathrm{q}(\mathrm{I}$ & DV|IV) & & \\
\hline \# & Rhy & Rhh & Rug & Rhe & $\operatorname{Rrd}$ & Rav & Rao & Rbn & Rse & Rnr & freq & $\mathrm{SNF}=1$ & $\mathrm{SNF}=2$ & $\mathrm{SNF}=1$ & $\mathrm{SNF}=2$ & & \\
\hline 1 & 0 & 0 & 0 & 0 & \begin{tabular}{|c|}
0 \\
\end{tabular} & 0 & 0 & 0 & 0 & 0 & 1371 & 92.34 & 7.66 & 92.15 & 7.85 & 0.55 & 0.00 \\
\hline 10 & 0 & 0 & 0 & 0 & 1 & 0 & 0 & 0 & 0 & 0 & 42 & 71.43 & 28.57 & 74.60 & 25.40 & 1.78 & 0.04 \\
\hline 18 & 0 & 0 & 0 & 1 & 0 & 0 & 0 & 0 & 0 & 1 & 49 & 73.47 & 26.53 & 68.93 & 31.07 & 2.18 & 0.00 \\
\hline 20 & 0 & 0 & 0 & 1 & 0 & 0 & 0 & 1 & 0 & 0 & 43 & 79.07 & 20.93 & 75.02 & 24.98 & 1.75 & 0.04 \\
\hline 22 & 0 & 0 & 0 & 1 & 0 & 0 & 1 & 0 & 0 & 0 & 26 & 76.92 & 23.08 & 72.01 & 27.99 & 1.96 & 0.05 \\
\hline 26 & 0 & 0 & 0 & 1 & 0 & 1 & 0 & 0 & 0 & 0 & 10 & 50.00 & 50.00 & 44.64 & 55.37 & 3.88 & 0.00 \\
\hline 50 & 1 & 0 & 0 & 0 & 1 & 0 & 0 & 0 & 0 & 0 & 14 & 78.57 & 21.43 & 59.33 & 40.67 & 2.85 & 0.01 \\
\hline 53 & 1 & 0 & 0 & 1 & 0 & 0 & 0 & 0 & 0 & 0 & 171 & 74.85 & 25.15 & 75.84 & 24.16 & 1.69 & 0.00 \\
\hline 56 & 1 & 0 & 0 & 1 & 0 & 0 & 0 & 1 & 0 & 0 & 12 & 66.67 & 33.33 & 59.87 & 40.13 & 2.81 & 0.01 \\
\hline & & & & & & & & & & & 3204 & 85.74 & 14.26 & 85.74 & 14.26 & & \\
\hline
\end{tabular}

Table 116. Condensed Component Fit Table for Comorbidity IVs in (Hip)

Best Model, Rhy SNF : Rhh SNF : Rug SNF :Rhe SNF : Rrd SNF :

Rav Rbn SNF : Rao SNF : Rse SNF : Rnr SNF.

Orange rows indicate ratio $>1.10$.

(Frequencies $<10$ and ratios between 0.91 and 1.09 are close to the margins and are excluded.)

\begin{tabular}{|c|c|c|c|c|c|c|}
\hline & & Data & \multicolumn{2}{|c|}{ obs. p(DV|IV) } & & \\
\hline IV & State & freq & SNF=1 & SNF=2 & Ratio & p(margin) \\
\hline Rhy & 1 & 386 & 74.61 & 25.39 & 1.78 & 0.00 \\
\hline Rhh & 1 & 10 & 30.00 & 70.00 & 4.91 & 0.00 \\
\hline Rug & 1 & 48 & 66.67 & 33.33 & 2.34 & 0.00 \\
\hline Rhe & 1 & 1464 & 81.83 & 18.17 & 1.27 & 0.00 \\
\hline Rrd & 1 & 77 & 63.64 & 36.36 & 2.55 & 0.00 \\
\hline Rao & 1 & 86 & 65.12 & 34.88 & 2.45 & 0.00 \\
\hline Rse & 1 & 11 & 36.36 & 63.64 & 4.46 & 0.00 \\
\hline Rnr & 1 & 99 & 68.69 & 31.31 & 2.20 & 0.00 \\
\hline \multicolumn{7}{r}{} \\
\hline
\end{tabular}

Table 117. Component Fit Table for Interaction Rav Rbn in (Hip)

Best Model Rhy SNF : Rhh SNF : Rug SNF :Rhe SNF : Rrd SNF :

Rav Rbn SNF : Rao SNF : Rse SNF : Rnr SNF.

Orange rows indicate ratio $>1.10$.

(Frequencies $<10$ and ratios between 0.91 and 1.09 are close to the margins and are excluded.)

\begin{tabular}{|c|c|c|c|c|c|c|c|}
\hline & \multicolumn{2}{|c|}{ IVs } & Data & \multicolumn{2}{c|}{ obs. p(DV|IV) } & & \\
\hline$\#$ & Rav & Rbn & freq & SNF=1 & SNF=2 & Ratio & p(margin) \\
\hline 1 & 0 & 1 & 147 & 72.11 & 27.89 & 1.96 & 0.00 \\
\hline 2 & 1 & 0 & 25 & 40.00 & 60.00 & 4.21 & 0.00 \\
\hline & & & 3204 & 85.74 & 14.26 & 1.00 & \\
\hline
\end{tabular}

